

\section{Propositions}

1. Northern Europe must prepare for WestNile virus transmission. (this thesis)

2. West Nile virus can affect the brain of both its vertebrate host and mosquito vector.

(this thesis)

3. Conformism is a poor evolutionary strategy.

4. The individual-based character of the scientific review process makes publishing a lottery.

5. Sufficient sleep is key to a successful career.

6. Benefits of doing sports outweigh the risks of injury.

Propositions belonging to the thesis, entitled

'The role of Culex pipiens mosquitoes in transmission of West Nile virus in Europe'

Chantal B.F. Vogels

Wageningen, 8 September 2017 

The role of Culex pipiens mosquitoes in transmission of West Nile virus in Europe

Chantal B.F. Vogels 
Thesis committee

\section{Promotor}

Prof. Dr M. Dicke

Professor of Entomology

Wageningen University \& Research

\section{Co-promotor}

Dr C.J.M. Koenraadt

Assistant professor, Laboratory of Entomology

Wageningen University \& Research

\section{Other members}

Prof. Dr R.A.A. van der Vlugt, Wageningen University \& Research

Dr M.A.H. Braks, National Institute for Public Health and the Environment, Bilthoven

Dr L.S. van Overbeek, Wageningen University \& Research

Dr W.F. de Boer, Wageningen University \& Research

This research was conducted under the auspices of the C.T. de Wit Graduate School for Production Ecology \& Resource Conservation 


\title{
The role of Culex pipiens mosquitoes in transmission of West Nile virus in Europe
}

\author{
Chantal B.F. Vogels
}

\section{Thesis}

submitted in fulfilment of the requirements for the degree of doctor at Wageningen University by the authority of the Rector Magnificus,

Prof. Dr A.P.J. Mol, in the presence of the

Thesis Committee appointed by the Academic Board to be defended in public on Friday 8 September 2017 at 4 p.m. in the Aula. 
Chantal B.F. Vogels

The role of Culex pipiens mosquitoes in transmission of West Nile virus in Europe, 212 pages.

$\mathrm{PhD}$ thesis, Wageningen University, Wageningen, the Netherlands (2017)

With references, with summaries in English and Dutch

ISBN 978-94-6343-615-1

DOI http://dx.doi.org/10.18174/418217 




\section{Table of contents}

Abstract $\quad 9$

Chapter 1

Introduction

Chapter 2

23

Winter activity and aboveground hybridization between the two biotypes of the West Nile virus vector Culex pipiens

\section{Chapter 3}

Latitudinal diversity of Culex pipiens biotypes and hybrids in farm, peri-urban, and wetland habitats in Europe

\section{Chapter 4}

Vector competence of northern European Culex pipiens biotypes and hybrids for West Nile virus is differentially affected by temperature

\section{Chapter 5}

Vector competence of northern and southern European Culex pipiens pipiens mosquitoes for West Nile virus across a gradient of temperatures

\section{Chapter 6}

Virus interferes with host-seeking behaviour of mosquito

\section{Chapter 7}

Modelling West Nile virus transmission risk in Europe: effect of temperature and mosquito biotypes on the basic reproduction number

\section{Chapter 8}

Vector competence of European mosquitoes for West Nile virus

\section{Chapter 9}

General discussion: Identification of mosquito-related factors that limit West Nile virus transmission in northern Europe

\section{References}

Summary

Samenvatting

Acknowledgements

Curriculum vitae 


\section{Abstract}

West Nile virus (WNV) is maintained in a natural transmission cycle between mosquito vectors and bird hosts. However, mosquitoes can also transmit WNV to mammals, such as humans and horses, which may result in disease. In Europe, such cases of WNV disease are yearly recurring in southern and central Europe, but have not been detected in northern Europe. The absence of WNV outbreaks in northern Europe may be explained by lowered vector competence of the main vector for WNV: the northern house mosquito, Culex (Cx.) pipiens. The aim of this thesis was, therefore, to investigate the role of $C x$. pipiens mosquitoes in transmission of WNV in Europe, in order to understand differences in WNV circulation between northern and southern Europe.

The species $C x$. pipiens consists of two biotypes, pipiens and molestus, which can form hybrids. Both biotypes and their hybrids are morphologically identical but differ in their behaviour, which may have consequences for WNV transmission. In this thesis, the Cx. pipiens biotype composition was investigated in The Netherlands, and more broadly at the European scale. These studies revealed that both biotypes and their hybrids are present throughout The Netherlands, and that there is a shift towards relatively more biotype pipiens mosquitoes at northern latitudes in Europe. The next step was to assess vector competence of these northern European biotypes and their hybrids, and to make a direct comparison with vector competence of a southern European population. Both biotypes and their hybrids originating from The Netherlands are competent vectors for WNV. Interestingly, no differences in vector competence were found between a Dutch and Italian biotype pipiens population. However, both studies revealed that low temperatures of $18{ }^{\circ} \mathrm{C}$ are an important limiting factor for WNV transmission. A more in-depth study on the effects of WNV on the host-seeking behaviour of biotype pipiens mosquitoes revealed that WNV infection reduces the host-seeking response towards host odour, but that other fitness parameters (e.g. flight activity, blood-feeding, and survival) are not affected. When results from the biotype composition and vector competence studies were included in a newly developed $R_{0}$ model, it becomes clear that biotype pipiens is the main contributor to WNV transmission.

The results presented in this thesis show that the Cx. pipiens biotypes and their hybrids are present throughout The Netherlands, and that they can transmit WNV under favourable climatic conditions. The absence of WNV outbreaks in northern Europe can most likely be explained by low temperature which has a negative effect on mosquito abundance, vector competence, and the duration of the infectious period. When considering the outcomes of this thesis in the light of climate change, northern European countries such as The Netherlands should be prepared for future WNV transmission. 

Chapter 1

Introduction 


\section{Emerging arthropod-borne viruses}

Arthropod-borne (arbo)viruses deploy a fascinating evolutionary strategy, by alternating replication in vertebrate and invertebrate hosts (Weaver 2006). As a consequence, most arboviruses have complex transmission cycles involving arthropod vectors, which transmit the virus from one vertebrate host to another (Weaver \& Barrett 2004). Transmission cycles can only be established under suitable climatic conditions, because virus replication is influenced by temperature (Hardy et al. 1983). Mosquitoes are one of the most important vectors for arboviruses of medical and veterinary importance. The majority of medically important arboviruses transmitted by mosquitoes, such as dengue, chikungunya, and Zika virus, are circulating in the tropics (Weaver \& Reisen 2010). However, globalisation and climate change have contributed to the emergence of arboviruses in more temperate regions, including Europe (Kilpatrick \& Randolph 2012, Morse 1995).

During the past decades several mosquito-borne viruses have been introduced in Europe, causing diseases in humans (Calistri et al. 2010a, Tomasello \& Schlagenhauf 2013). Of these viruses, West Nile virus (WNV; family: Flaviviridae) can be considered the mosquito-borne virus of highest medical and veterinary importance for Europe, due to the recurrent number of cases in humans and equines every summer (ECDC 2016). WNV is most notorious for its rapid spread throughout the United States of America (USA; Hayes et al. 2005). Since the introduction in New York in 1999, WNV spread from the East to the West coast within 5 years (Hayes et al. 2005). In contrast, such rapid spread has not been observed in Europe, where cases of WNV in humans have been limited to southern and central European countries (Figure 1; ECDC 2016). Thus far, no cases of WNV in humans have been reported in northern Europe. Differences in WNV circulation between northern and southern Europe may possibly be explained by host-related, vector-related, and environmental factors, or the interaction between these factors. The aim of this thesis was to investigate the role of mosquitoes in transmission of WNV in Europe, in order to understand differences in WNV circulation between northern and southern Europe.

\section{West Nile virus}

WNV is maintained in a natural transmission cycle between birds and mosquitoes (Figure 2). Mosquitoes can transmit WNV to mammals, including humans and equines, which may result in disease. However, in general mammals do not develop high enough virus levels in their blood (viraemia) to support further transmission to mosquitoes (Hayes et al. 2005). Therefore, they are considered "dead-end" hosts. An estimated 80 percent of human infections with WNV remain asymptomatic, and, thus, many WNV cases remain undetected or unreported. Symptomatic cases 
mostly develop into fever and less than 1 percent of cases develops neurological disease, such as encephalitis (Mostashari et al. 2001). Currently, there is no vaccine or specific treatment against WNV available for humans. From 1999 to 2016, over 46,000 cases of WNV human disease, and approximately 2,000 deaths have been reported in the USA (CDC 2015). In contrast, a few hundred human disease cases are yearly reported in Europe (ECDC 2016).

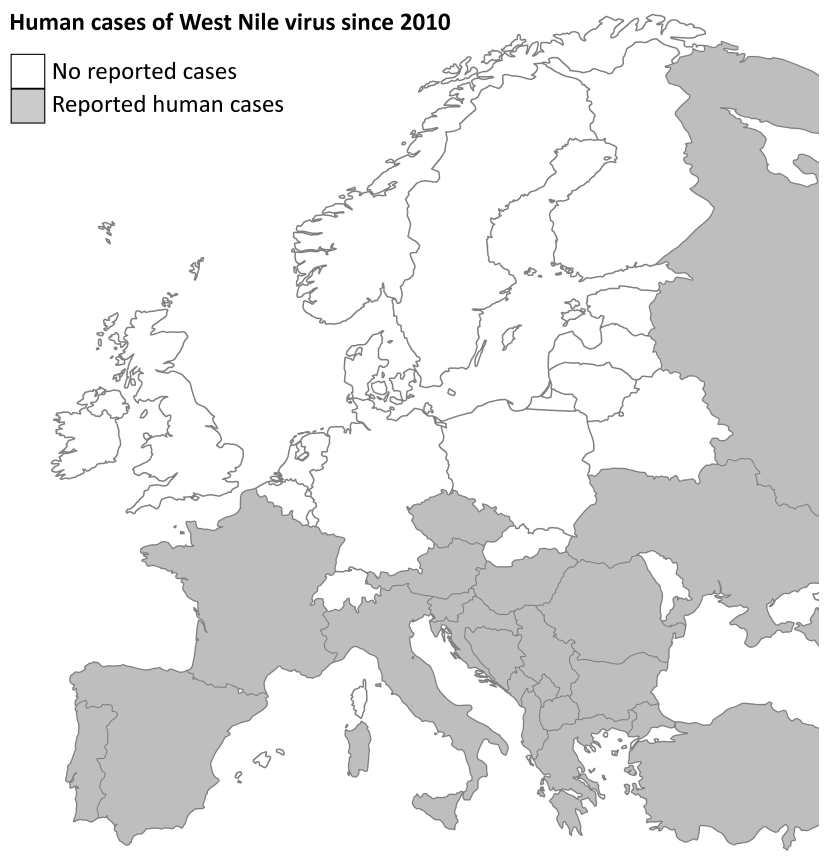

Figure 1: European countries with reported cases of West Nile virus infection in humans. Countries marked in grey have experienced human cases of West Nile virus since 2010. Countries marked in white have not reported any cases of West Nile virus in humans. Dataset provided by ECDC, based on data provided by WHO and Ministries of Health from the affected countries (ECDC 2016).

WNV was discovered for the first time in Uganda in 1937 (Smithburn et al. 1940). Since its discovery, WNV has spread around the world, and can now be found on all continents except Antarctica (Chancey et al. 2015). The presence of WNV in Europe was already reported in 1958 from Albany, based on the detection of WNV-specific antibodies in humans (Bardos et al. 1959). This was followed by reports of WNV cases in humans from France in the 1960s (Hubalek \& Halouzka 1999, Murgue et al. 2001). Since then, yearly recurring outbreaks have occurred in southern and central Europe, and WNV can now be considered endemic in the southern and central parts of Europe (Figure 1; Calistri et al. 2010a, Sambri et al. 2013). 


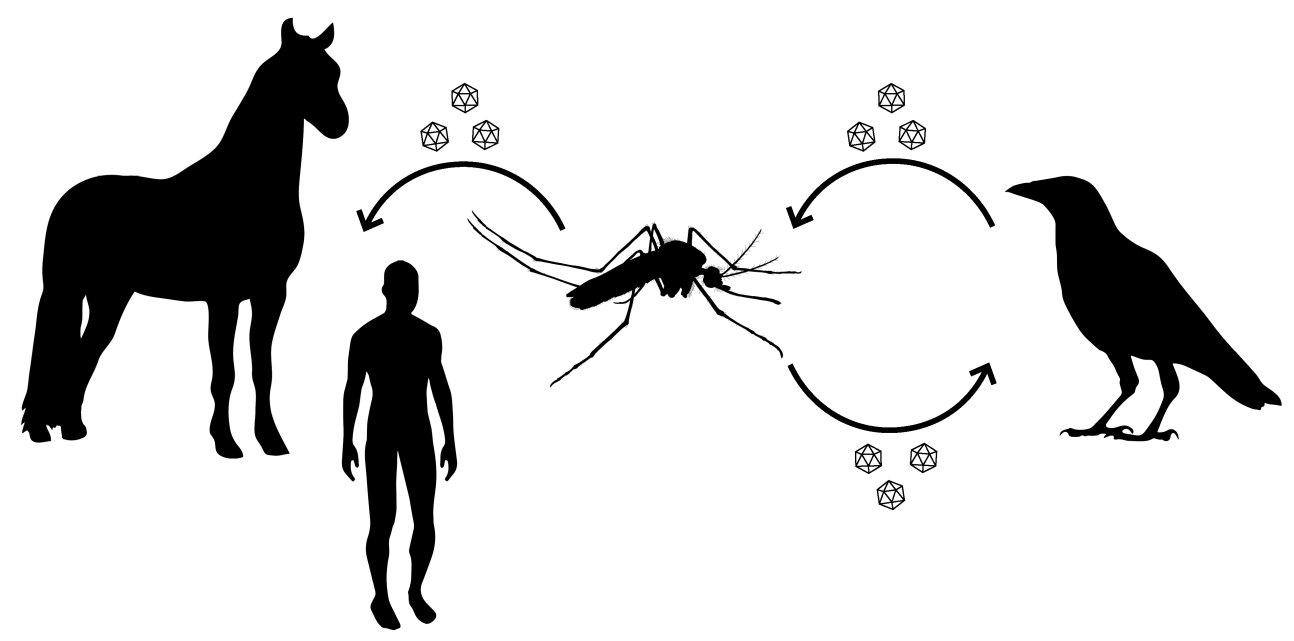

Figure 2: Natural transmission cycle of West Nile virus and spill-over to humans and equines. The West Nile virus transmission cycle involves mosquito vectors and bird hosts. Humans and equines may get infected with West Nile virus, which can result in disease, but they do not develop high enough viraemia for further transmission to mosquitoes.

WNV has a single-stranded positive-sense RNA genome of approximately 11,000 nucleotides, and can be divided in at least two major viral lineages which have been associated with human disease (Lanciotti et al. 2002). Although the current taxonomic status needs further confirmation, at least five additional viral lineages have been proposed which differ up to $25 \%$ from the two major lineages (reviewed in Pesko \& Ebel 2012). The initial outbreaks of WNV in Europe were caused by virus strains belonging to lineage 1 (Calistri et al. 2010a). Lineage 1 can be subdivided into three clades which each consist of virus strains with a specific geographic distribution (Figure 3). The European WNV strains belong to the lineage 1a clade, together with strains circulating in North America, Africa, and Asia (Lanciotti et al. 2002). The other two lineage 1 clades include the Australian Kunjin virus strains (clade 1b), and Indian WNV strains (clade 1c). In 2004, WNV lineage 2 was identified for the first time in Europe (Bakonyi et al. 2006). It has been speculated that migratory birds that overwintered in Central Africa introduced the WNV lineage 2 strain into Europe. Since 2008, WNV lineage 2 rapidly spread across central Europe and was responsible for a WNV outbreak in Greece in 2010 (Papa et al. 2011). 


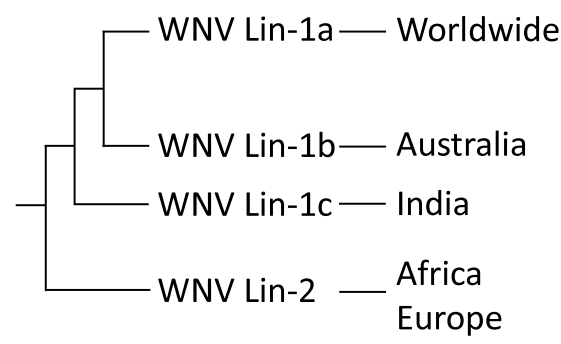

Figure 3: Schematic overview of phylogenetic relationships between the two main West Nile virus lineages and their geographic distribution. Each West Nile virus lineage or clade consists of several virus strains with a specific geographic distribution. Lineage 1 consists of three clades with either a worldwide distribution (lineage 1a), or more specific distribution in Australia (lineage 1b), or India (lineage 1c). Lineage 2 virus strains circulate in Africa, but have more recently also been found in Europe. Phylogeny based on (Lanciotti et al. 2002, Pesko \& Ebel 2012).

\section{Vertebrate hosts of West Nile virus}

From 1999 to 2012, WNV has been detected in dead birds of more than 300 species in the USA (CDC 2012b, Komar 2003). Outbreaks of WNV in humans in the USA were preceded by high mortality of corvid birds, especially the American crow (Corvus brachyrhynchos; Eidson et al. 2001a, Eidson et al. 2001b). However, it was not the American crow but the American robin (Turdus migratorius) that was identified as the major contributor to WNV transmission (Hamer et al. 2009, Kent et al. 2009, Kilpatrick et al. 2006). This major contribution was due to disproportionally high feeding rates of competent mosquito species on the American robin, even at low bird abundance. In Europe, death rates among birds associated with WNV seem much lower compared to North America (Calistri et al. 2010b, Dauphin et al. 2004, Jourdain et al. 2007b, Valiakos et al. 2011). Moreover, it remains unclear which bird species contribute most to the natural WNV cycle in Europe.

Reduced host susceptibility of northern European bird populations has been proposed as a possible explanation for the lack of WNV outbreaks in northern Europe. Thus far, two laboratory studies investigated the susceptibility of two bird species in The Netherlands. Both the carrion crow (Corvus corone) and European jackdaw (Corvus monedula) were found to be susceptible to WNV (Lim et al. 2015, Lim et al. 2014). Although it was questionable whether viraemia of European jackdaws was sufficient to support transmission (Lim et al. 2014), viraemia of carrion crows could support transmission (Lim et al. 2015). These laboratory findings are supported by field studies carried out in northern Europe, which reported serological evidence of WNV in sentinel chickens, resident birds, and migratory birds in the United Kingdom (Buckley et al. 2006, Buckley et al. 2003), and in migratory birds in Germany (Linke et al. 2007). This evidence may suggest that WNV is already circulating among birds 
in northern Europe, although actual evidence in the form of detection of infectious WNV is still lacking. Further laboratory and field studies are required to gain more insight into the role of northern European birds in WNV transmission. However, these first results indicate that birds are not likely the limiting factor for WNV transmission in northern Europe.

\section{Mosquitoes as vectors of West Nile virus}

Mosquitoes play a central role in the transmission of WNV, because they are responsible for transmission between hosts (Komar 2003, Turell et al. 2005). From 1999 to 2012, WNV has been detected in more than 60 mosquito species in the USA (CDC 2012a). However, not all mosquitoes that ingest WNV will actually be able to transmit the virus. This is due to several barriers inside the mosquito: the midgut infection and escape barrier, and the salivary gland infection and escape barrier (Franz et al. 2015, Hardy et al. 1983). These barriers strongly determine the vector competence, i.e. the ability of a vector to become infected and transmit a pathogen (Kenney \& Brault 2014). Only those mosquito species in which WNV is able to overcome all barriers and infect the saliva, are considered competent vectors and can contribute to the transmission cycle. Vector competence is mainly determined by intrinsic factors of the vector (e.g. barriers and antiviral responses) and extrinsic factors (e.g. temperature; Franz et al. 2015, Hardy et al. 1983). Consequently, vector competence for WNV has been shown to vary on a spatial and temporal scale (Kilpatrick et al. 2010, Vaidyanathan \& Scott 2007). Reduced vector competence of northern European mosquitoes, potentially driven by lower temperature, has been proposed as an alternative hypothesis which may explain the absence of WNV outbreaks in northern Europe.

It is important to realize that vector competence is not the only factor that determines the efficiency with which vectors can transmit a pathogen in a host population. This efficiency is essentially defined as vectorial capacity, and includes other vectorrelated factors such as survival, biting rate, and the ratio between vectors and hosts (Garrett-Jones 1964a, Garrett-Jones 1964b). All components of vectorial capacity need to be taken into account when identifying the main vector species for a certain pathogen. This can be illustrated by the fact that a highly competent mosquito species may not be the most efficient vector in the field, for instance due to a feeding preference for hosts that are not susceptible or due to a relative low abundance of the vector. Based on both laboratory and field studies, the northern house mosquito, Culex (Cx.) pipiens, has been identified as one of the most important vectors for WNV (Fonseca et al. 2004). Culex pipiens mosquitoes are competent vectors for WNV in laboratory studies (Balenghien et al. 2008, Kilpatrick et al. 2008, Turell et al. 2001a), 
they have a relatively high infection prevalence in the field (Engler et al. 2013, Hayes et al. 2005), strong preference to feed on bird hosts (Fritz et al. 2015, Hamer et al. 2009, Osório et al. 2014), and high abundance during summer (Chaskopoulou et al. 2016, Spielman 2001), which together makes Cx. pipiens a potentially important contributor to WNV transmission (Kilpatrick et al. 2005). Therefore, this thesis will focus on the role of $C x$. pipiens in transmission of WNV in Europe.

There is an ongoing debate on which species should be included in the Cx. pipiens complex (Fonseca et al. 2004). The northern house mosquito (Cx. pipiens Linnaeus $1758)$ and southern house mosquito (Cx. quinquefasciatus Say 1823) are formally recognized as members of the complex. Cx. pipiens mosquitoes are mainly found in temperate regions, including Europe, and $C x$. quinquefasciatus in more tropical regions, with hybridization between both species in zones where the geographical distributions overlap (Vinogradova 2000). Thus far, no consensus has been reached on whether or not other closely related species such as $C x$. autralicus, $C x$. pipiens pallens, Cx. torrentium, and $C x$. vagans belong to the $C x$. pipiens complex (Harbach 2012, Smith \& Fonseca 2004, Vinogradova 2000). From all the potential members of the complex, only $C x$. pipiens and $C x$. torrentium can be found in Europe.

The species $C x$. pipiens can be further divided into two morphologically identical biotypes, pipiens (Linnaeus 1758) and molestus (Forskål 1775), which show distinct behaviour(Vinogradova2000). The pipiens biotype prefers birds as host (ornithophilic), diapauses during winter, mates in swarms (eurygamous), and requires a blood meal prior to egg laying (anautogenous; Byrne \& Nichols 1999). In contrast, the molestus biotype prefers mammals as host (mammophilic), remains active year-round, mates in confined spaces (stenogamous), and can lay its first egg batch without a blood meal (autogenous; Marshall \& Staley 1935, Shute 1941, Willcox 1941). The two biotypes can hybridize, and hybrids show intermediate behaviour. Due to their more opportunistic feeding behaviour, hybrids are considered important bridge vectors which can transmit WNV from birds to humans (Fritz et al. 2015, Kilpatrick et al. 2005). Behavioural differences between biotypes may have implications for their contribution to the WNV transmission cycle. Thus, differentiation between biotypes is important when investigating the role of $C x$. pipiens in transmission of WNV in Europe.

Already in the 1950's, initial ideas regarding the origin of the Cx. pipiens biotypes were proposed. This has resulted in two main hypotheses (Mattingly et al. 1951). The first hypothesis proposes that biotype molestus diverged from the pipiens biotype when adapting to underground environments during multiple colonization events. The second hypothesis states that biotype pipiens derived from a northern Cx. pipiens species with the typical pipiens traits such as anautogeny, whereas 
biotype molestus derived from a southern species originating from southern Europe or northern Africa which was autogenous. After decades of research, evidence mostly points to a common origin of biotype molestus populations, which supports the second hypothesis (Fonseca et al. 2004, Gomes et al. 2009, Vinogradova \& Shaikevich 2007, Weitzel et al. 2009).

Despite the presence of susceptible bird hosts (Lim et al. 2015, Lim et al. 2014), and high abundance of one of the main mosquito vectors, Cx. pipiens, during summer (Hesson et al. 2013, Ibañez-Justicia et al. 2015, Rudolf et al. 2013, Weitzel et al. 2011), WNV outbreaks have not yet occurred in northern Europe. The question, therefore, remains which factors are limiting the transmission of WNV in northern Europe. Answers to this question may be found in differences between northern and southern European Cx. pipiens populations. Not only biotype composition may play an important role in the establishment of WNV transmission cycles, but also differences in vector competence between $C x$. pipiens populations, which can be influenced by intrinsic factors or environmental conditions.

\section{Aim of this thesis}

The aim of this thesis was to elucidate the role of $C x$. pipiens mosquitoes in transmission of WNV in Europe, and to identify factors that can explain the differences in WNV circulation between northern and southern Europe. This thesis focuses on mosquitoes as key players in the transmission of WNV, by investigating the interaction between mosquito, virus, and temperature (Figure 4).

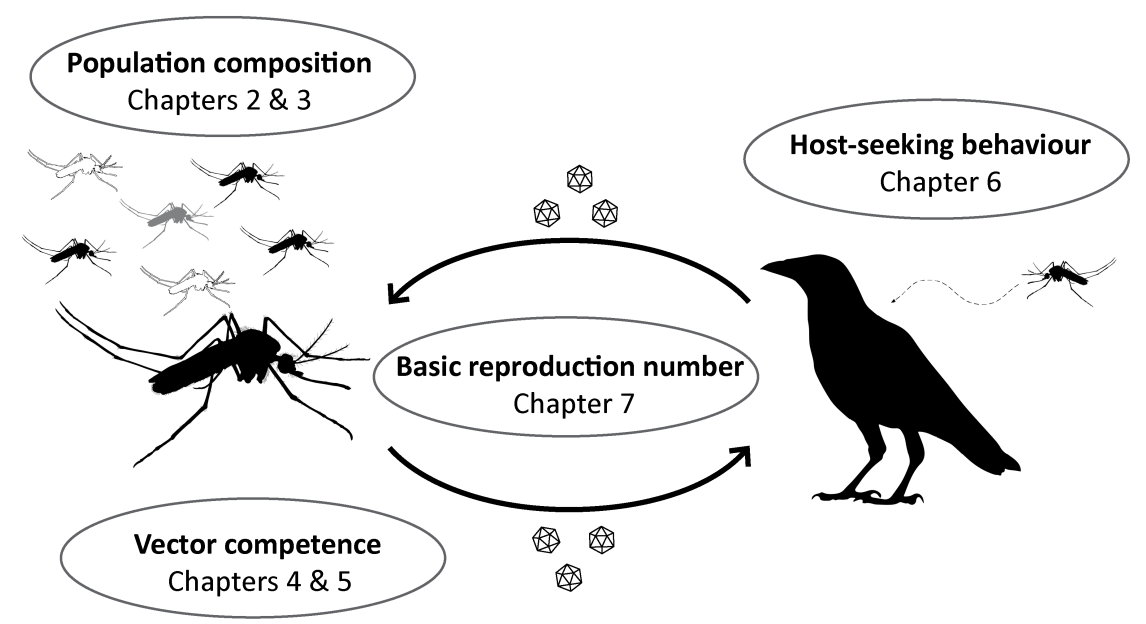

Figure 4: Graphical overview of this PhD thesis. Overview of the experimental chapters of this thesis and their link to the West Nile virus transmission cycle. Colours of mosquitoes represent the different Culex pipiens biotypes and hybrids, with biotype pipiens: black, biotype molestus: white, and hybrids: grey. 


\section{Outline of the thesis}

Chapter 2 presents the spatial distribution of $C x$. pipiens in The Netherlands. Central in this chapter is the differentiation between the two $C x$. pipiens biotypes and their hybrids. Mosquitoes were collected through two complementary strategies: traditional mosquito surveillance and an alternative and newly launched citizenscience approach (Muggenradar). By combining data from the traditional mosquito surveillance with the citizen-science approach, year-round mosquito collections could be compared to gain insight in the presence and biting activity of the $C x$. pipiens biotypes and hybrids in The Netherlands.

Chapter 3 addresses the diversity of the $C x$. pipiens biotypes, by investigating the biotype composition at the European scale. Effects of habitat on the biotype composition were investigated across different latitudes in Europe, including sampling locations in Sweden, The Netherlands, and Italy. Comparison of the biotype compositions at different latitudes shows a clear shift in biotype composition from northern to southern Europe, which may have implications for WNV transmission.

Chapters 4 and 5 examine the ability of European $C x$. pipiens mosquitoes to transmit WNV. In chapter 4 , vector competence of the $C x$. pipiens biotypes originating from The Netherlands is investigated at different temperatures. The aim of this chapter was to investigate the role of temperature as a potentially limiting factor for WNV transmission by mosquitoes in northern Europe. Chapter $\mathbf{5}$ directly compares vector competence of a northern and southern European $C x$. pipiens biotype pipiens population, at representative temperatures. This chapter investigates whether differences in WNV outbreaks between northern and southern Europe can be explained by reduced vector competence of northern European mosquito populations.

Chapter 6 presents an in-depth study on the interaction between WNV and its mosquito vector, by investigating the consequences of WNV infection on the mosquito's behaviour and performance. The aim of this chapter was to investigate whether WNV can increase its transmission by influencing the host-seeking behaviour and performance of its mosquito vector.

Chapter 7 compiles results from the previous chapters on the distribution of the $C x$. pipiens biotypes (chapter 3 ) and their vector competence (chapter 4 ), by including them in a newly developed basic reproduction number $\left(R_{0}\right)$ model. The novelty of this $R_{0}$ model is the inclusion of biotype-specific and temperature-dependent parameters. With these specific parameters the risk of WNV establishment and contribution of the biotypes to WNV transmission were assessed for several scenarios that are relevant for Europe. 
Chapter 8 describes the current state of knowledge on vector competence of European mosquito species for WNV, by comparing results from chapters 4 and 5 with other studies on European mosquitoes. This comparative approach is complemented with detailed insights in the underlying mechanisms of vector competence, such as the mosquito's antiviral response and microbiome, which may explain observed patterns in vector competence. This review evaluates whether vector competence can explain differences in WNV circulation between northern and southern Europe, and identifies knowledge gaps that need to be addressed in future research.

Chapter 9 integrates the results of this thesis into a broader context, by identifying mosquito-related factors that are most important for WNV establishment. Results obtained in this thesis will be discussed in the light of the theoretical concept of the basic reproduction number. Based on this concept, factors will be identified that are currently limiting for transmission of WNV in northern Europe. Finally, current and future risks for WNV transmission in northern Europe will be evaluated.

\section{Acknowledgements}

I thank Marcel Dicke and Sander Koenraadt for providing comments on an earlier version of this chapter. 



\section{Chapter 2}

\section{Winter activity and aboveground hybridization between the two biotypes of the West Nile virus vector Culex pipiens}

Chantal B.F. Vogels ${ }^{1}$, Lennart J.J. van de Peppel ${ }^{1}$, Arnold J.H. van Vliet $^{2}$, Marcel Westenberg $^{3}$, Adolfo Ibañez-Justicia ${ }^{4}$, Arjan Stroo ${ }^{4}$, Jan A. Buijs ${ }^{5}$, Tessa M. Visser ${ }^{1}$, and Constantianus J.M. Koenraadt ${ }^{1}$

${ }^{1}$ Laboratory of Entomology, Wageningen University \& Research

${ }^{2}$ Environmental Systems Analysis Group, Wageningen University \& Research

${ }^{3}$ Dutch National Plant Protection Organization, Netherlands Food and Consumer Product Safety Authority (NVWA)

${ }^{4}$ Centre for Monitoring of Vectors (CMV), National Reference Centre (NRC), Netherlands Food and Consumer Product Safety Authority (NVWA)

${ }^{5}$ Public Health Service Amsterdam

Vector-borne and Zoonotic Diseases (2015) 15: 619-626 


\begin{abstract}
Culex (Cx.) pipiens mosquitoes are important vectors of West Nile virus (WNV). The species $C x$. pipiens consists of two biotypes, pipiens and molestus, which are morphologically identical, but differ in behaviour. Typical behaviour of the molestus biotype is the ability to remain active during winter, whereas the pipiens biotype enters diapause. The current paradigm is that the two biotypes occur sympatrically in southern Europe, but occur in isolated above- and belowground populations in northern Europe. In northern Europe, hybridization between biotypes is considered to be low because of the barrier that exists between typical habitats. Data on the occurrence of the biotypes and hybrids in northern Europe, however, are scarce, because identification to the level of biotype is often not performed. Our objective was to clarify the distribution of the $C x$. pipiens biotypes and to determine hybridization rates in The Netherlands. Culex pipiens mosquitoes were collected using three different approaches. Firstly, traps were deployed randomly throughout The Netherlands during the summers of 2011 and 2012 (active surveillance). Secondly, using a web-based reporting platform and media campaign, Dutch citizens were asked to send dead mosquitoes to our laboratory during the winter and summer of 2014 (passive surveillance). Thirdly, larvae and adults were collected during the summer of 2014 from aboveground locations in Amsterdam, in order to identify molestus larval habitats. Real-time PCR was used for identification to the level of biotype. We found that biotype molestus and hybrids are feeding indoors during winter and summer in The Netherlands, and that hybridization rates ranged between 6-15\%. Larval habitats of biotype molestus were found to occur aboveground. The high percentage of hybridization has implications for assessing the risk of WNV transmission, because hybrids are thought to have ideal characteristics for bridging WNV between birds and humans.
\end{abstract}

Keywords: distribution, pipiens, molestus, Culex torrentium, Europe, Netherlands, active surveillance, passive surveillance 


\section{Introduction}

West Nile virus (WNV) is one of the most broadly distributed arthropod-borne viral diseases in the world (Kramer et al. 2008). To date, however, no cases of WNV have been detected in northern Europe, while WNV outbreaks occur in southern Europe (Calistri et al. 2010a, Hubalek \& Halouzka 1999, Reusken et al. 2010b, Sambri et al. 2013). The emergence of WNV in the United States of America (USA) after its introduction in 1999, has shown the potential of WNV to spread rapidly in a wide range of temperate climates (Davis et al. 2005, Ebel et al. 2004, Snapinn et al. 2007). The question remains, therefore, why has the virus not spread to northern Europe?

Culex (Cx.) pipiens mosquitoes have been identified as important vectors of WNV in the USA (Fonseca et al. 2004, Kilpatrick et al. 2005). The species, Cx. pipiens, consists of two biotypes named pipiens (Linnaeus 1758) and molestus (Forskål 1775), which are identical in morphology but which have different genetic backgrounds and show distinct behaviour. The pipiens biotype occurs aboveground, prefers birds as hosts (ornithophilic), mates in swarms (eurygamous), requires a blood meal to lay eggs (anautogenous) and diapauses during winter (Byrne \& Nichols 1999). In contrast, the molestus biotype occurs belowground (e.g. in underground tunnels), prefers mammals as hosts (mammophilic), mates in confined spaces (stenogamous), does not require a blood meal to lay the first egg batch (autogenous) and remains active during winter (Marshall \& Staley 1935, Shute 1941, Willcox 1941). Host availability also plays an important role in the selection for hosts. Approximately $90 \%$ of females of the mammophilic molestus biotype that were collected from inside chicken coops in Portugal, had blood-fed on birds (Gomes et al. 2013b). Hybrids between the pipiens and molestus biotypes are hypothesized to have intermediate behavioural traits and this may favour the transmission of WNV from birds to humans (Fonseca et al. 2004, Kilpatrick et al. 2005). Hybridization, however, is considered to be low in northern Europe due to separation of the typical habitats of the two biotypes (Fonseca et al. 2004). If hybrids indeed play a key role in WNV transmission, then the absence of hybrids could be one of the possible explanations for the absence of WNV cases in humans in northern Europe.

Although several studies have measured the spatial distribution of $C x$. pipiens and the related species $C x$. torrentium (Martini 1925) in Europe, most of them did not differentiate to the biotype level (Hesson et al. 2011, Hesson et al. 2013, Weitzel et al. 2011). The view of spatially separated populations of the two biotypes (Fonseca et al. 2004), therefore, may not reflect the real situation in northern Europe, because differentiation between biotypes was not addressed in many studies. Rudolf et al. (2013) performed analyses to the biotype level and found that the molestus and 
pipiens biotypes occur sympatrically in Germany. No strong conclusions on the occurrence of biotype molestus or hybrid frequencies could be drawn, however, because samples were analysed in pools and habitats were not systematically analysed (Rudolf et al. 2013).

The main objective of the current study was to elucidate the ecology of the biotypes of $C x$. pipiens and their hybrids in northern Europe, with a focus on the spatial and seasonal distribution in The Netherlands. Developing our understanding of the occurrence of the different $C x$. pipiens biotypes and hybrids is essential for WNV risk assessments, because not all mosquitoes are expected to be equally competent vectors or have equal inherent characteristics for bridging WNV to humans.

\section{Materials and Methods}

\section{Adult mosquito collection: active surveillance throughout The Netherlands}

The Centre for Monitoring of Vectors operates an annual nationwide mosquito surveillance programme in The Netherlands. The detailed protocol and outcomes of this programme have been published elsewhere (Ibañez-Justicia et al. 2015). In brief, mosquitoes were sampled from mid-April until the second half of October in both 2011 and 2012, using $\mathrm{CO}_{2}$-baited Mosquito Magnet Liberty plus traps (Woodstream Corp., USA; hereafter MM-trap) for 375 randomly selected locations in The Netherlands (Ibañez-Justicia et al. 2015). At each location, one trap was run for 7 days and thereafter the contents of the traps were collected and sent to the laboratory. Mosquitoes were morphologically identified based on the Culicidae key specifically designed for rapid field-identification of adult Culicidae (Becker et al. 2010, Schaffner et al. 2001, Snow 1990, Verdonschot 2002). At 353 locations $(94.1 \%)$ mosquitoes were caught, of which in total 3,363 Cx. pipiens/torrentium mosquitoes at 236 (62.9\%) of these locations. At least one $C x$. pipiens/torrentium sample from each of 158 locations was available and in good condition for further molecular analysis. One mosquito of each of these locations (2011: $n=82,2012: n$ $=76$ ) was, therefore, selected for further analysis to the species and biotype level by real-time PCR (see below).

\section{Adult mosquito collection: passive surveillance throughout The Netherlands}

A different and unique sampling method was developed for indoor mosquito collections, involving the participation of Dutch citizens (Kampen et al. 2015). A website was launched through which Dutch citizens were asked to fill out a questionnaire regarding mosquito presence and possible nuisance (www.muggenradar.nl). At the end of the questionnaire the respondents were requested to send a dead mosquito 
specimen to the Laboratory of Entomology of Wageningen University. A unique code made it possible to link the information obtained through the questionnaire with the mosquito that was submitted. In order to obtain indoor samples from winter and summer, the project was run for 5 weeks during January and February 2014 (average temperature of $\left.6.1^{\circ} \mathrm{C}(\mathrm{KNMI} 2014)\right)$ and for 2 weeks during August and September 2014 (average temperature of $16.0^{\circ} \mathrm{C}$ (KNMI 2014)). Mosquitoes were identified to the species level based on morphology following the above described Culicidae key. A selection of $150 \mathrm{Cx}$. pipiens/torrentium samples, divided over two groups, was made of the samples that were sent in by citizens during the winter of 2014. Mosquitoes in the first group $(n=68)$ had caused self-reported nuisance and by visual inspection upon arrival in the laboratory were confirmed to be bloodfed (active during winter), whereas mosquitoes in the second group $(n=82)$ had not caused nuisance and were not engorged. For the samples submitted during summer, a selection based on the same criteria, of in total $172 \mathrm{Cx}$. pipiens/torrentium samples was made. Mosquitoes in the first group $(n=89)$ had caused nuisance and by visual inspection were confirmed to be blood-fed, whereas mosquitoes in the second group $(n=83)$ had not caused nuisance and were not engorged. All selected mosquitoes were further analysed by real-time PCR (see below).

\section{Larval and adult mosquito collection: in-depth study of the city of Amsterdam}

The highest number of mosquito reports came from Amsterdam and this triggered further investigation. Astratified random sampling design $(1 \times 1 \mathrm{~km})$ was used to select 94 locations in Amsterdam. From May to September 2014, all publicly accessible larval habitats were identified within a radius of 50 meter around each selected location. This resulted in 133 habitats where mosquito larvae were found. Fourth instar larvae were identified to the species level based on morphology following Becker et al. (2010). In total 119 larval habitats contained Cx. pipiens larvae. From a selected number of 48 habitats all larvae $(n=480)$ were identified to the biotype level by real-time PCR (see below). In addition, from July to September 2014 MM-traps were placed at a selection of 20 out of the 94 locations for a duration of 2 weeks. All Cx. pipiens/torrentium adults captured in these traps $(n=161)$ were also identified to the biotype level by real-time PCR.

\section{Molecular identifications: DNA extraction}

DNA was extracted by heating mosquito legs in $100 \mu \mathrm{l}$ of $4 \mathrm{M}$ ammoniumhydroxide for $20 \mathrm{~min}$ at $99{ }^{\circ} \mathrm{C}$, cooling on ice for $1 \mathrm{~min}$, centrifuging (20 s at $\left.3700 \mathrm{rpm}\right)$ and heating again for another $20 \mathrm{~min}$ at $90^{\circ} \mathrm{C}$ with the lids open (Guy \& Stanek 1991). DNA extracts were stored in the freezer at $-20^{\circ} \mathrm{C}$ until further analysis. 


\section{Molecular identifications: primer and probe design}

The real-time PCR assay as described by Rudolf et al. (2013) was adjusted to optimize the design, because amplification curves did not all show an exponential increase and did not reach the same plateau, which made the interpretation of results difficult. DNA sequences of microsatellite CQ11 of $C x$. pipiens biotype pipiens, biotype molestus, $C x$. pallens, and $C x$. quinquefasciatus were mined from GenBank and aligned with Geneious R7 (Biomatters, New Zealand) using MAFFT (Katoh et al. 2002). Based on the alignments new primers were designed with the use of Primer Express V3.0 software (Life technologies, The Netherlands). Cx_pip_F (5'-GCGGCCAAATATTGAGACTTTC-3') and Cx_pip_R (5'-ACTCGTCCTCAAACATCCAGACATA-3') were designed as universal $C x$. pipiens forward and reverse primers, respectively. For identification of biotype molestus, probe Cpp_mol_P (5'-FAM-TGAACCCTCCAGTAAGGTA-MGB-3') was designed, which is a shorter version of the original probe by Rudolf et al. (2013), conjugated with a minor groove binder (MGB) group. The two probes Cpp_pip_ P1 (5'-VIC-CACACAAAYCTTCACCGAA-MGB-3') and Cpp_pip_P2 (5'-VICACACAAACCTTCATCGAA-MGB-3') were designed for identification of biotype pipiens to cover the sequence variation at the original probe binding site. Hybrids can be identified by the presence of amplification curves for both the pipiens and molestus probes.

DNA sequences of acetylcholinesterase 2 (ace-2) from Culex spp. were mined from GenBank and aligned for $C x$. torrentium identification. Based on the original primers and probes of Rudolf et al. (2013), forward and reverse primers were enlarged at the 5' end to obtain Cx_tor_F (5'-CTTATTAGTATGACACAGGACGACAGAAA-3') and Cx_tor_R (5'-GCATAAACGCCTACGCAACTACTAA-3'). The probe was shortened at the 5' end and conjugated with a MGB group to get Cx_tor_P (5'-FAMATGATGCCTGTGCTACCA-MGB-3').

\section{Molecular identifications: real-time PCR}

Real-time PCR tests were performed in two separate reactions (one for each locus) and consisted of 1x Premix Ex Taq (Takara Bio, Japan), $300 \mathrm{nM}$ of each primer, and $100 \mathrm{nM}$ dual labelled hydrolysis probe, except for the Cpp_pip1 for which $200 \mathrm{nM}$ was added. Molecular grade water and $2 \mu$ DNA template were added to reach a final volume of $25 \mu$. Thermocycler conditions were $95^{\circ} \mathrm{C}$ for $10 \mathrm{~min}$, followed by 45 cycles of $95{ }^{\circ} \mathrm{C}$ for $15 \mathrm{~s}$ and $62{ }^{\circ} \mathrm{C}$ for $1 \mathrm{~min}$. Real-time PCR runs were performed in 96-well plates on a CFX96 Real-Time PCR system (Bio-Rad Laboratories, USA) and data were analysed in CFX manager 2.0. Samples with Cq values below 40 and with real-time PCR curves showing exponential increase were considered positive. 
Positive amplification controls for each target organism and negative amplification controls were included in each real-time PCR run.

\section{Molecular identifications: validation}

The new real-time PCR design was validated with at least ten specimens from each line of morphologically and behaviourally well characterized $C x$. torrentium (Best, The Netherlands), biotype pipiens (Pennsylvania, USA), biotype molestus (Bologna, Italy) and lab-reared hybrids between the latter two.

\section{Distribution maps}

Arc-GIS 10.0 (Environmental Systems Research Institute, USA) was used to generate maps.

\section{Results}

\section{Molecular identifications: validation}

The real-time PCR was initially performed with an elongation temperature (Ta) of $60{ }^{\circ} \mathrm{C}$. The obtained amplification curves for the characterized samples all showed exponential increases that matched the corresponding species or biotype, and reached more or less the same plateau for each fluorophore. Culex torrentium, however, gave a false positive signal with the biotype pipiens probes at high $\mathrm{C}_{t}$ (>39) values, although not with an exponential curve. Therefore a real-time PCR with a gradient $\left(\mathrm{Ta}=60-63^{\circ} \mathrm{C}\right)$ was performed which had an optimum $\mathrm{Ta}$ of $62^{\circ} \mathrm{C}$ at which the false amplification curve for $\mathrm{Cx}$. torrentium remained below the automatic threshold, while other curves were not affected.

\section{Adult mosquito collection: active surveillance throughout The Netherlands}

Of the total number of 158 analysed samples from the National Vector Survey, 104 samples were identified as biotype pipiens (65.8\%), 39 as biotype molestus (24.7 $\%$ ), ten as hybrids (6.3\%), and five as $C x$. torrentium (3.2\%; Figure 1 ). 


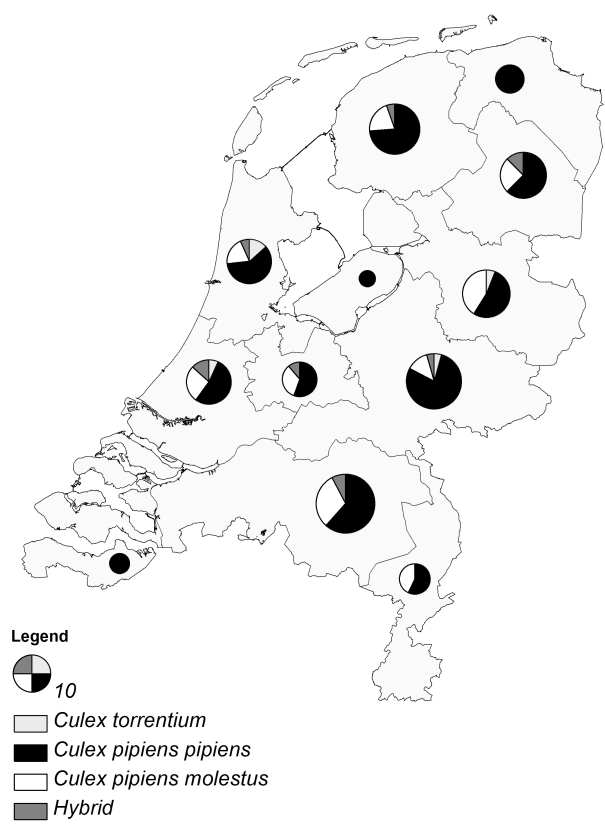

Figure 1: Culex mosquitoes collected outdoors with Mosquito Magnet Liberty plus traps during the summers of 2011 and 2012 in The Netherlands. Relative composition of trapped Culex mosquitoes that were identified to the species and biotype level per province. The size of each pie chart varies according to the number of analysed samples.

\section{Adult mosquito collection: passive surveillance throughout The Netherlands}

In total 1,563 mosquitoes were submitted during winter, from which 930 were identified as Culex mosquitoes. Out of $68 \mathrm{Cx}$. pipiens/torrentium mosquitoes that were blood-fed and had caused nuisance as reported by Dutch citizens from indoor locations during the winter of 2014 , one was identified as pipiens (1.5\%), 60 as molestus (88.2 \%), seven as hybrids (10.3\%), and none as Cx. torrentium (0\%; Figure 2A). In contrast, out of 82 non-nuisance mosquitoes that were submitted during winter, 58 were identified as pipiens (70.7\%), 11 as molestus (13.4\%), 12 as hybrids (14.6\%), and one as $C x$. torrentium (1.2\%; Figure $2 \mathrm{~B})$. During summer, in total 581 mosquitoes were submitted, from which 397 were identified as Culex mosquitoes. From the total number of 89 mosquitoes that were blood-fed and had caused nuisance indoors during summer, six were identified as pipiens $(6.7 \%)$, 62 as molestus (69.7\%), 21 as hybrids (23.6\%), and none as Cx. torrentium (0 $\%$; Figure $2 \mathrm{C}$ ). In contrast, from the total number of 83 non-nuisance mosquitoes that were submitted during summer, 48 were identified as pipiens (57.8\%), 30 as molestus (36.1\%), five as hybrids $(6.0 \%)$, and none as $C x$. torrentium (0\%; Figure 2D). 
A

Nuisance

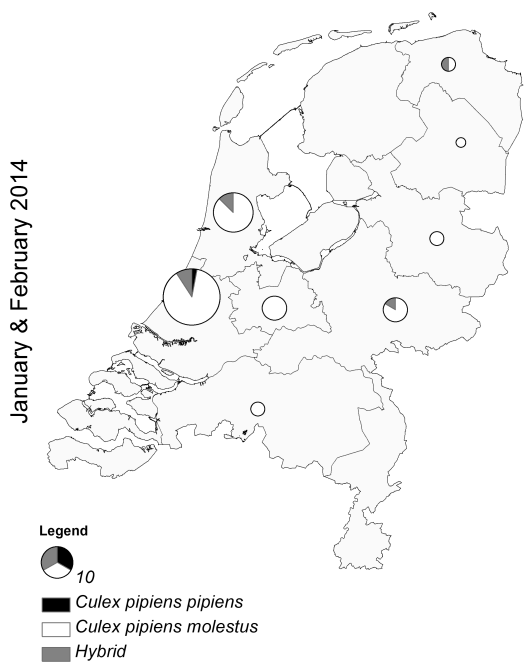

C

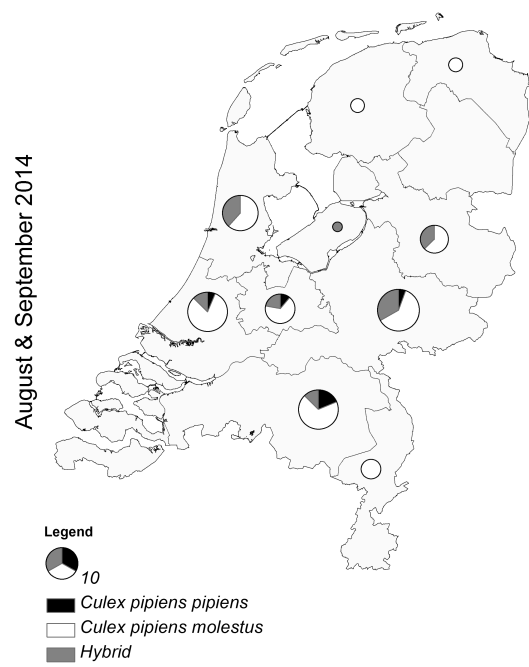

B No nuisance
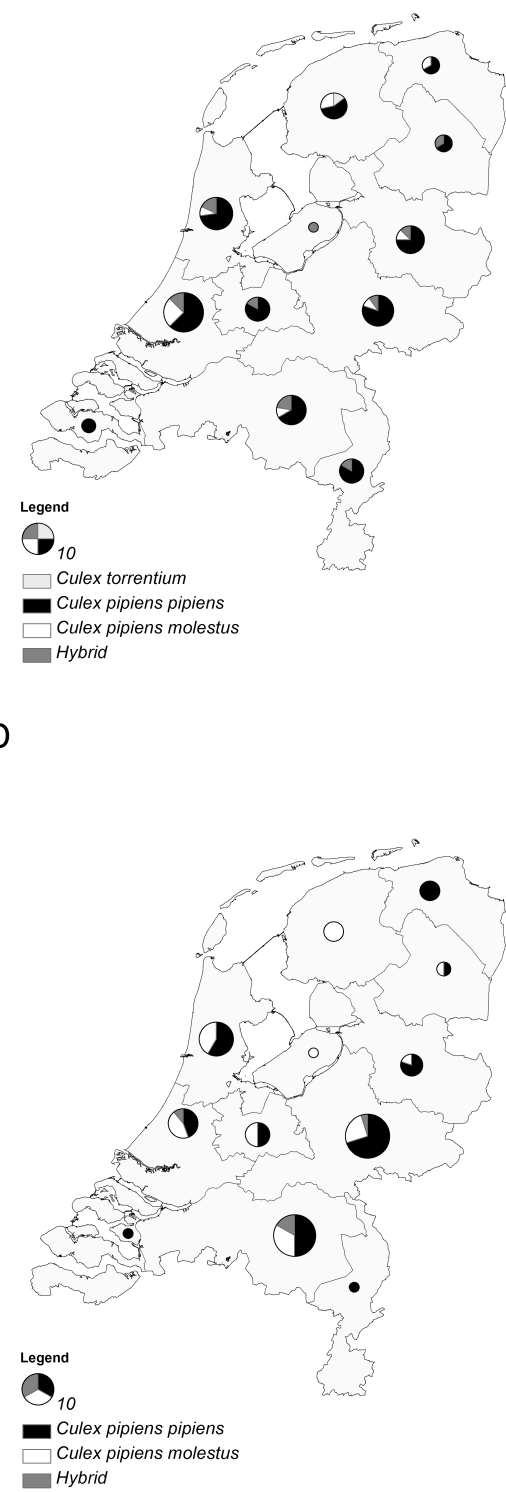

Figure 2: Culex mosquitoes collected indoors by Dutch citizens during the winter (January and February) and summer (August and September) of 2014 in The Netherlands. Relative composition of Culex mosquitoes per province, collected during January and February 2014, (A) that had caused nuisance and were engorged, and (B) that had not caused nuisance. Relative composition of Culex mosquitoes per province, collected during August and September 2014, (C) that had caused nuisance and were engorged, and (D) that had not caused nuisance. The size of each pie chart varies according to the number of analysed samples. 


\section{Larval and adult mosquito collection: in-depth study of the city of Amsterdam}

From the $480 \mathrm{Cx}$. pipiens larvae that were analysed, 436 were identified as pipiens (90.8 \%), 19 as molestus (4.0\%), and 25 as hybrids (5.2\%; Figure 3). These larvae were collected from a total number of 48 publicly accessible aboveground larval habitats, such as rainwater storm drains, man-made rainwater collections and ditch water. In total 26 larval habitats (54.2\%) contained only biotype pipiens larvae, one larval habitat $(2.1 \%)$ contained only biotype molestus larvae, one larval habitat (2.1 $\%$ ) contained only hybrid larvae, and 20 of the larval habitats (41.6\%) contained a mixture of biotypes and/or hybrids.

From the total number of $161 \mathrm{Cx}$. pipiens/torrentium adults that were collected in Amsterdam, 121 were identified as pipiens (75.2\%), 26 as molestus $(16.1 \%)$, and 14 as hybrids ( $8.7 \%$; Figure 3$)$. These results are similar to the nationwide results of active surveillance.

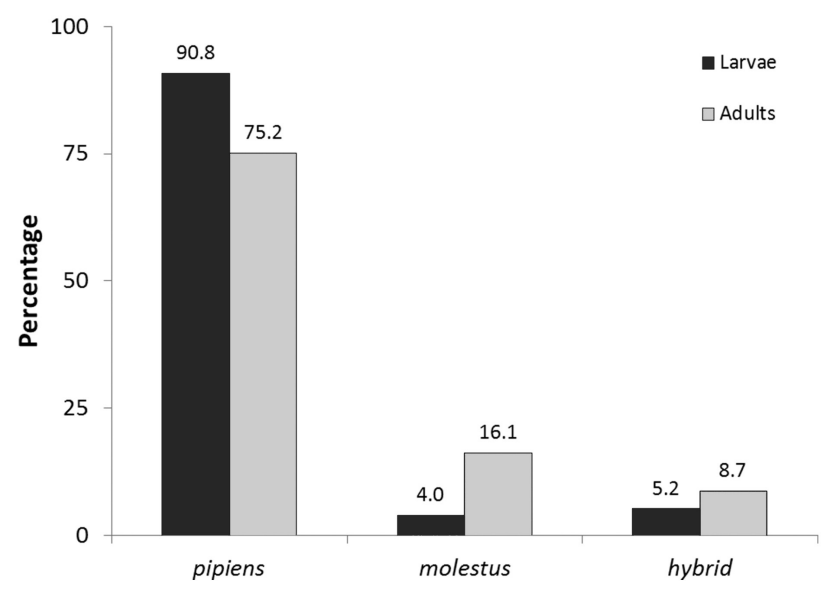

Figure 3: Percentage of Culex pipiens larvae and adults collected aboveground in Amsterdam. Culex pipiens larvae $(n=480)$ collected at 48 larval habitats, and Culex pipiens adults $(n=161)$ collected at 20 locations with MM-traps, were identified to biotype level with real-time PCR. Dark-grey bars represent larvae, and light-grey bars represent adults. Values indicate exact percentages.

\section{Discussion}

The analysis of blood-fed specimens that had been submitted to our laboratory by members of the Dutch public suggests that both molestus and hybrids continue to feed indoors during winter, and are mainly responsible for indoor bites year-round. Moreover, we found a high frequency of aboveground hybridization between the Cx. pipiens biotypes in The Netherlands. In contrast to the current paradigm that 
the molestus biotype is restricted to belowground habitats in northern Europe, we found that the pipiens and molestus biotypes occur sympatrically in aboveground locations.

In the view of biotypes occupying spatially separated habitats, it can be expected that hybridization is low (Chevillon et al. 1995, Fonseca et al. 2004). Hybrids of the two biotypes were described from The Netherlands in 2010, but they were exclusively reported from the underground transit system of Amsterdam (Reusken et al. 2010a). In the present nationwide study, we found that biotypes pipiens and molestus both occur aboveground (in mosquito traps, as well as inside houses of Dutch citizens), as is the case in southern European countries, with comparable hybridization rates ranging between 5-15\% (Amraoui et al. 2012, Gomes et al. 2013a, Gomes et al. 2009, Osório et al. 2014).

Not only adult molestus and hybrid mosquitoes were collected aboveground in this study, aquatic habitats containing molestus and hybrid larvae were also found to exist aboveground. Interestingly, relatively few larval habitats with biotype molestus were identified in comparison with biotype pipiens. The percentage of biotype molestus was higher for adults collected at the same locations in Amsterdam, than for larvae. It is likely that a fraction of the typical underground biotype molestus habitats were not sampled, such as water collection basins in the underground (Reusken et al. 2010a). In addition to a bias in sampling of the larval populations, habitat characteristics and environmental conditions at trapping locations could have influenced the number and composition of collected adult mosquitoes (DiMenna et al. 2006, Giberson et al. 2007). An alternative explanation could be that biotype pipiens larvae are better competitors and, therefore, can outcompete biotype molestus larvae.

It remains unclear whether the molestus biotype has only recently expanded its niche from belowground to aboveground habitats or whether it has been aboveground for longer periods of time but was overlooked in previous studies. Locations where biotype molestus was found were distributed throughout the country. Therefore, we hypothesize that this is not a new development but that it has been aboveground for a longer period of time, or has always been present. This is supported by the collections of molestus from hotels and stagnant water in the United Kingdom by Willcox in the mid twentieth century (Willcox 1941).

The lack of exponential increase in the amplification curves of well characterized samples tested with the real-time PCR protocol developed by Rudolf et al. (2013) is probably due to competition between the ' $C x$. pipiens all' and the 'biotype pipiens' probes, as these were overlapping. Additionally, we found several single nucleotide mismatches between the biotype pipiens probe and sequences in GenBank. These issues were resolved by designing new primers and probes. 
Passive surveillance with the help of citizens complements active surveillance tools that involve the use of traps (Kampen et al. 2015). Samples obtained through passive surveillance can provide specific insight into which mosquito species are present inside houses. Using this approach, our study revealed that biotype molestus was mostly responsible for biting nuisance, especially during winter. It is likely that during this season biotype pipiens has entered diapause and may thus be observed by citizens (Sim \& Denlinger 2008), but not experienced as nuisance. The numerous reports submitted via the website revealed that nuisance biting during winter is not uncommon. Such insights cannot be obtained by using traps. Traps, however, provide more insight into mosquito abundance, which is more difficult to achieve with passive surveillance.

To date, WNV has not been found to circulate among mosquitoes in a potentially high-risk area in The Netherlands, where both mosquitoes and wildlife are abundant (Reusken et al. 2010b). Because hybrids could play an important role in bridging WNV from birds to humans, the presence of year-round actively feeding hybrids in The Netherlands could constitute a risk, if WNV were to be introduced in The Netherlands. North-western European $C x$. pipiens mosquitoes have already been found to be competent vectors for WNV (Balenghien et al. 2008, Balenghien et al. 2007, Fros et al. 2015a). The question, therefore, remains which factors, other than the presence of competent vectors, restrict WNV from establishing in northern Europe. A partial explanation may be that viremia levels in birds, such as European jackdaws, remain too low to be picked up by mosquitoes (Lim et al. 2014). Our future research efforts will focus on the impact of temperature on mosquito-virus interactions (chapters $4 \& 5$ of this thesis).

\section{Conclusions}

Biotype molestus and hybrids are active and feeding indoors during both summer and winter and are mainly responsible for indoor biting nuisance. High levels of hybridization between $C x$. pipiens biotype pipiens and molestus are observed in The Netherlands, which can be explained by sympatric occurrence of both biotype pipiens and molestus in aboveground habitats.

\section{Acknowledgements}

We thank the Dutch citizens who sent mosquito samples to our laboratory. Our thanks go to Rens van den Biggelaar and Marian Dik for morphological identifications and colleagues from the Laboratory of Entomology, especially Linda Klein and Steffanie Teekema, for their help in processing all samples. We also thank Marcel Dicke and Alexandra Hiscox for providing comments on a previous version of this chapter. 



\section{Chapter 3}

\section{Latitudinal diversity of Culex pipiens biotypes and hybrids in farm, peri-urban, and wetland habitats in Europe}

Chantal B.F. Vogels ${ }^{1 *}$, Tim W.R. Möhlmann ${ }^{1,2^{*}}$, Diede Melsen ${ }^{1}$, Guido Favia ${ }^{3}$, Uno Wennergren ${ }^{2}$, and Constantianus J.M. Koenraadt ${ }^{1}$

* These authors contributed equally to this work

${ }^{1}$ Laboratory of Entomology, Wageningen University \& Research

2 IFM Theory and Modelling, Linköping University

${ }^{3}$ School of Biosciences and Veterinary Medicine, University of Camerino

PLoS One (2016) 11: e0166959 


\begin{abstract}
Despite the presence of Culex (Cx.) pipiens mosquitoes and circulation of West Nile virus (WNV), WNV outbreaks have so far not occurred in northern Europe. The species Cx. pipiens consists of two morphologically identical biotypes, pipiens and molestus, which can form hybrids. Until now, population dynamic studies of $C x$. pipiens have not differentiated between biotypes and hybrids at the European scale, nor have they used comparative surveillance approaches. We therefore aimed to elucidate the relative abundance of $C x$. pipiens biotypes and hybrids in three habitat types at different latitudes across Europe, using two different surveillance traps. BG-Sentinel and Mosquito-Magnet Liberty Plus traps were placed in three habitat types (farms, peri-urban, wetlands), in three European countries (Sweden, The Netherlands, Italy). Collected Cx. pipiens mosquitoes were identified to biotype 3 with real-time PCR. Both trap types collected equal ratios of the biotypes and their hybrids. From northern to southern latitudes there was a significant decrease of pipiens and an increase of molestus. Habitat types influenced the relative ratios of biotypes and hybrids, but results were not consistent across latitudes. Our results emphasize the need to differentiate $C x$. pipiens to the biotype level, especially for proper future WNV risk assessments for Europe.
\end{abstract}

Keywords: West Nile virus, population dynamics, northern house mosquito, biotype molestus 


\section{Introduction}

Global warming, increased travel and trade, and land-use changes are important drivers for the (re-)emergence of vector-borne diseases, such as West Nile virus (WNV; family: Flaviviridae; Morens et al. 2004). The potential of WNV to quickly spread to new areas is clearly illustrated by the outbreaks that occurred in the United States of America, after the initial introduction in 1999 (Davis et al. 2005, Ebel et al. 2004, Snapinn et al. 2007). WNV outbreaks have also occurred in southern and central European countries, but no outbreaks among humans have occurred in northern Europe (Calistri et al. 2010a, Hubalek \& Halouzka 1999, Sambri et al. 2013).

WNV is maintained in an enzootic cycle between birds and mosquitoes. The main vector for WNV is the mosquito Culex (Cx.) pipiens (Fonseca et al. 2004, Kilpatrick et al. 2005). The $C x$. pipiens complex consists of several closely related species and biotypes, of which only the species Cx. pipiens (Linnaeus 1758) occurs in Europe (Farajollahi et al. 2011). Because of its similar morphology, the species Cx. torrentium (Martini 1925) is often included in taxonomic studies of the $C x$. pipiens complex (Becker et al. 2012). The species Cx. pipiens consists of two morphologically similar biotypes, named pipiens (Linnaeus 1758) and molestus (Forskål 1775), which show distinct behaviour. Biotype pipiens is the most important vector in the enzootic cycle because of its preference for birds (Byrne \& Nichols 1999). During winter, biotype pipiens enters diapause, which provides a means of overwintering for WNV (Dohm \& Turell 2001, Sanburg \& Larsen 1973). Biotype molestus prefers mammals, including humans, as hosts, and remains active year-round (Marshall \& Staley 1935, Shute 1941, Willcox 1941). Host availability can induce a strong shift in host feeding behaviour of biotype molestus from mammals to birds, especially in areas with high bird densities (Gomes et al. 2013b). Previously, biotype molestus has been described as occurring underground (Fonseca et al. 2004), but recent studies show that both biotypes occur sympatrically in aboveground habitats throughout Europe (Di Luca et al. 2016, Osório et al. 2014, Rudolf et al. 2013, Vogels et al. 2015). Furthermore, biotype pipiens and biotype molestus can form hybrids which show intermediate host preference (Fritz et al. 2015). As a result of this, hybrids can play an important role in bridging WNV from birds to humans (Fonseca et al. 2004).

Several studies elucidated the geographic distribution of the species Cx. pipiens and Cx. torrentium (Martini 1925) at the European scale (Hesson et al. 2013, Weitzel et al. 2011). In general, $C x$. torrentium is relatively more abundant in northern Europe, whereas Cx. pipiens is more abundant in southern Europe (Hesson et al. 2013). However, these studies did not identify $C x$. pipiens mosquitoes to the biotype level. Identification to the biotype level is important because the behavioural differences 
between the two biotypes of $\mathrm{Cx}$. pipiens and their hybrids result in different vectorial capacity for WNV. Thus far, in-depth studies that differentiated between the biotypes were done at country level (Di Luca et al. 2016, Gomes et al. 2013a, Osório et al. 2014, Rudolf et al. 2013, Vogels et al. 2015, Zittra et al. 2016). Few of these studies systematically compared biotype ratios among different habitat types (Di Luca et al. 2016, Osório et al. 2014, Zittra et al. 2016). However, due to differences in experimental design it is hard to make direct comparisons between $C x$. pipiens populations in northern and southern European countries.

The aim of this study was to assess the relative abundance of the $C x$. pipiens biotypes with two types of traps (Biogents Sentinel and Mosquito Magnet Liberty Plus), in three different habitat types (farms, peri-urban, and wetlands), and in three countries (Sweden, The Netherlands, and Italy) at different latitudes across Europe.

\section{Materials and Methods}

\section{Ethics statement}

Permits and approval for field work in wetlands were obtained from the county board of Östergötland in Sweden, Staatsbosbeheer in The Netherlands, and the Protected Areas Service of the San Benedetto del Tronto Municipality in Italy. For farms and peri-urban habitats approval was obtained from landowners of private properties in all three countries. No protected species were sampled in this study.

\section{Mosquito collections}

Adult mosquitoes were collected with the Biogents Sentinel (BGS) trap (BioGents $\mathrm{GmbH}$, Germany) and the Mosquito Magnet Liberty Plus (MMLP) trap (Woodstream Corp., USA). A mixture of $17.5 \mathrm{~g}$ dry instant yeast (Bruggeman, The Netherlands), $250 \mathrm{~g}$ white granulated sugar and 2 I of tap water in a 5 I plastic bottle was used for $\mathrm{CO}_{2}$ production in the BGS trap (Smallegange et al. 2010). Combustion of propane provided $\mathrm{CO}_{2}$ for the MMLP trap.

Both traps were rotated among three trapping locations, in three different habitat types (farms, peri-urban, and wetlands), in Sweden (Linköping), The Netherlands (Wageningen), and Italy (San Benedetto del Tronto; Table 1). The selected farms were dairy cattle farms with a minimum of 10 cows. Traps were placed within 50 $\mathrm{m}$ of the open indoor stable. Peri-urban locations were at the periphery of a city (inhabitants $<150,000$ ), and within a $50 \mathrm{~m}$ radius of the trap, at least two occupied residential properties were present. Locations in a wetland habitat had a minimum of $50 \%$ marshy or standing water within a $100 \mathrm{~m}$ radius of the traps. Trapping locations were at least $100 \mathrm{~m}$ apart. 
Table 1: Coordinates of all 27 trapping locations in the three different habitat types (farms, periurban, and wetlands) in three different European countries (Sweden, The Netherlands, and Italy).

\begin{tabular}{|c|c|c|c|}
\hline Country & Habitat type & Sampling location & Coordinates \\
\hline Sweden & Farms & 1 & $58.296530,15.584782$ \\
\hline \multirow[t]{8}{*}{ (Linköping) } & & 2 & $58.343622,15.602404$ \\
\hline & & 3 & $58.330597,15.704327$ \\
\hline & Peri-urban & 4 & $58.416973,15.499516$ \\
\hline & & 5 & $58.401515,15.626744$ \\
\hline & & 6 & $58.405494,15.595035$ \\
\hline & Wetlands & 7 & $58.362106,15.651861$ \\
\hline & & 8 & $58.361585,15.654910$ \\
\hline & & 9 & $58.361542,15.659072$ \\
\hline The Netherlands & Farms & 10 & $51.971084,5.761455$ \\
\hline \multirow[t]{8}{*}{ (Wageningen) } & & 11 & $51.973637,5.773978$ \\
\hline & & 12 & $52.013077,5.645998$ \\
\hline & Peri-urban & 13 & $52.018075,5.655372$ \\
\hline & & 14 & $51.979257,5.645230$ \\
\hline & & 15 & $51.979771,5.660278$ \\
\hline & Wetlands & 16 & $51.969443,5.758940$ \\
\hline & & 17 & $51.967693,5.758896$ \\
\hline & & 18 & $51.971671,5.747826$ \\
\hline Italy & Farms & 19 & $42.914466,13.854588$ \\
\hline \multirow[t]{8}{*}{ (San Benedetto del Tronto) } & & 20 & $42.944809,13.859857$ \\
\hline & & 21 & $42.943098,13.853856$ \\
\hline & Peri-urban & 22 & $42.883455,13.879388$ \\
\hline & & 23 & $42.951012,13.850783$ \\
\hline & & 24 & $42.934424,13.891933$ \\
\hline & Wetlands & 25 & $42.896600,13.911895$ \\
\hline & & 26 & $42.899042,13.909813$ \\
\hline & & 27 & $42.903365,13.908667$ \\
\hline
\end{tabular}

Collections were done during six consecutive days, every month in each country. Sampling periods were from July 2014 to June 2015, except for the winter months December, January, and February (and March for Sweden). Traps were emptied and repositioned every 24 hours between sunrise and sunset of the next day. Mosquitoes were stored at $-20^{\circ} \mathrm{C}$ in Eppendorf tubes containing small silica beads covered with cotton wool. 


\section{Mosquito identifications}

All female mosquitoes were identified to species level, following the European identification key for female mosquitoes (Becker et al. 2010). The number of $C x$. pipiens mosquitoes captured each month was not sufficient to statistically test for temporal differences in biotype and hybrid ratios. Therefore, all $190 \mathrm{Cx}$. pipiens females available for analysis from Sweden, and a selection of $300 \mathrm{Cx}$. pipiens females from Italy and 299 Cx. pipiens females from The Netherlands were used, resulting in a total of 789 mosquitoes analysed. Samples from the Netherlands and Italy were partially random selected with 100 samples per habitat, for both countries.

Selected mosquitoes were further identified to species (Cx. pipiens or $C x$. torrentium) and biotype (pipiens, molestus, or hybrid) level. We followed the real-time PCR assay for differentiation between the $C x$. pipiens biotypes as described in detail before (Vogels et al. 2015). Briefly, for Cx. pipiens we used forward and reverse primers Cx_pip_F (5'-GCGGCCAAATATTGAGACTTTC-3') and Cx_pip_R (5'-ACTCGTCCTCAAACATCCAGACATA-3'). For identification of biotype molestus we used probe Cpp_mol_P (5'-FAMTGAACCCTCCAGTAAGGTA-MGB-3'), and for biotype pipiens we used the two probes Cpp_pip_P1 (5'-VIC-CACACAAAYCTTCACCGAA-MGB-3') and Cpp_ pip_P2 (5'-VIC-ACACAAACCTTCATCGAA-MGB-3'). Hybrids were identified when both probes for biotype pipiens and molestus were amplified by real-time PCR. For identifications of $C x$. torrentium we used forward and reverse primers Cx_tor_F (5'-CTTATTAGTATGACACAGGACGACAGAAA-3') and Cx_tor_R (5'-GCATAAACGCCTACGCAACTACTAA-3'), and probe Cx_tor_P (5'-FAMATGATGCCTGTGCTACCA-MGB-3'). Thermocycler conditions were $95{ }^{\circ} \mathrm{C}$ for 10 min, followed by 45 cycles of $95^{\circ} \mathrm{C}$ for $15 \mathrm{~s}$ and $62{ }^{\circ} \mathrm{C}$ for $1 \mathrm{~min}$. The PCR was run on the CFX96 Real-Time PCR system (Bio-Rad Laboratories, USA) and data were analysed in CFX manager 2.0 (Bio-Rad Laboratories).

\section{Statistical analysis}

Main effects (trap type, country, and habitat) and within-effects (habitats within each country, and country within each habitat) on the ratios of $C x$. pipiens mosquitoes were tested with Pearson's Chi-square tests. Significant effects were further evaluated with pairwise comparisons and corrected with the Bonferroni correction. All data were analysed in the statistical software package R (R Core Team 2016).

\section{Results}

In total 5,202 Cx. pipiens females were collected of which 3,878 females were collected with the BGS trap and 1,324 females with the MMLP trap (Table 2). Of 
the 789 mosquitoes selected for analysis, 663 mosquitoes were identified as $C x$. pipiens, of which $463(69.8 \%)$ were identified as biotype pipiens, 127 (19.2\%) as biotype molestus, and $73(11.0 \%)$ as hybrids. In addition, 14 mosquitoes were identified as $C x$. torrentium, which all originated from Sweden. The number of $C x$. torrentium mosquitoes was too low for reliable statistical tests, and these samples were therefore excluded from further analyses. The remaining 112 mosquitoes did not amplify a PCR product.

Table 2: Total number of collected Culex pipiens females per trap type, habitat, and country. BGS $=$ Biogents Sentinel trap, MMLP = Mosquito Magnet Liberty Plus trap, SW $=$ Sweden, NL $=$ The Netherlands, and IT = Italy.

\begin{tabular}{lcccccccc}
\hline & \multicolumn{3}{c}{ BGS } & & \multicolumn{3}{c}{ MMLP } \\
\cline { 2 - 4 } \cline { 7 - 8 } & SW & NL & IT & & SW & NL & IT \\
\hline Farms & 19 & 252 & 128 & & 29 & 64 & 21 \\
Peri-Urban & 56 & 1063 & 111 & & 44 & 451 & 37 \\
Wetlands & 33 & 969 & 1247 & & 24 & 101 & 553 \\
\hline Total & 108 & 2284 & 1486 & & 97 & 616 & 611 \\
\hline
\end{tabular}

Both trap types, BGS and MMLP, trapped similar ratios of the $C x$. pipiens biotypes and hybrids $\left(X^{2}=2.35, \mathrm{df}=2, P=0.31\right.$; Figure $\left.1 \mathrm{~A}\right)$. Thus, data from both trap types were pooled for further analyses.

The ratios of $C x$. pipiens biotypes and hybrids were significantly different between the three countries in Europe $\left(X^{2}=173.62, d f=4, P<0.001\right.$; Figure 1B). Pairwise comparisons between countries showed that $C x$. pipiens ratios were different between each combination of Italy, The Netherlands, and Sweden (all pairwise comparisons: $P<0.001$ ). The proportion of biotype pipiens was highest in Sweden $(90 \%)$ and gradually decreased towards more southern latitudes, with the lowest proportion of biotype pipiens in Italy (40\%).

The ratios of $C x$. pipiens biotypes and hybrids were also significantly different between habitat types $\left(X^{2}=26.59, \mathrm{df}=4, P<0.001\right.$; Figure $\left.1 C\right)$. Peri-urban habitats had a relatively higher proportion of biotype pipiens compared to both farms $(P<$ $0.01)$, and wetlands $(P<0.001)$. There was no difference in ratios between farms and wetlands $(P=0.16)$. 

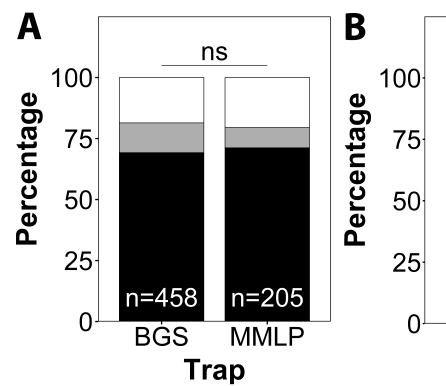

Cx. p. molestus

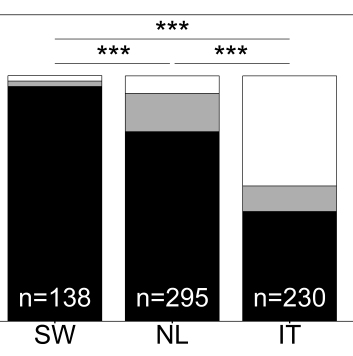

Country

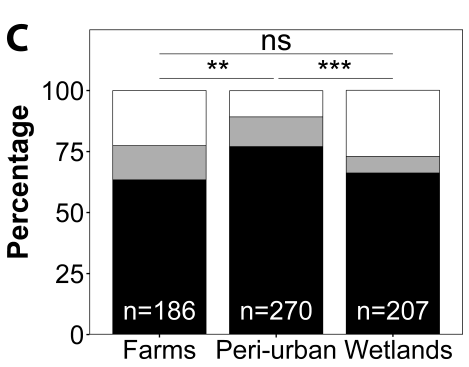

Habitat

Figure 1: Main effects of (A) trap type, (B) country, and (C) habitat on the ratio of Culex pipiens biotypes and their hybrids. The total sample size $(n)$ is indicated for each bar. Significance is displayed for each pairwise comparison, with ns $=$ not significant, ${ }^{* *}=p<0.01,{ }^{* * *}=p<0.001$. BGS $=$ Biogents Sentinel trap, MMLP = Mosquito Magnet Liberty Plus, $\mathrm{SW}=$ Sweden, NL $=$ The Netherlands, and IT = Italy.

In order to gain more insight in the interaction between country and habitat, pairwise comparisons were made between the habitats within each country, and the countries within each habitat type (Figure 2). Ratios of $C x$. pipiens biotypes and hybrids were significantly different between habitats in Italy $\left(x^{2}=25.05\right.$, df $\left.=4, P<0.001\right)$ and The Netherlands $\left(X^{2}=26.37, \mathrm{df}=4, P<0.001\right)$, but were similar within Sweden $\left(X^{2}=\right.$ 6.11, df = 4, $P=0.19$; Figure 2). In The Netherlands, farms were different due to the relatively high proportion of biotype molestus and hybrids $(P<0.01)$, whereas in Italy wetlands were different due to the high proportion of biotype molestus $(P<0.001)$.

Ratios of $C x$. pipiens biotypes and hybrids were significantly different between countries within each of the habitat types $(P<0.001$; Figure 2). Farms in Sweden had a relatively higher proportion of biotype pipiens compared to Italy $(P<0.001)$ and The Netherlands $(P<0.01)$, which both had relatively more biotype molestus and hybrids. For peri-urban habitats, ratios were significantly different among all countries (pairwise comparisons: $P \leq 0.001$ ), with a gradual increase of biotype pipiens towards northern latitudes. Wetlands in Italy had a relatively low proportion of biotype pipiens but high proportion of biotype molestus, compared to The Netherlands $(P<0.001)$ and Sweden $(P<0.001)$, which both had relatively high proportions of biotype pipiens. 


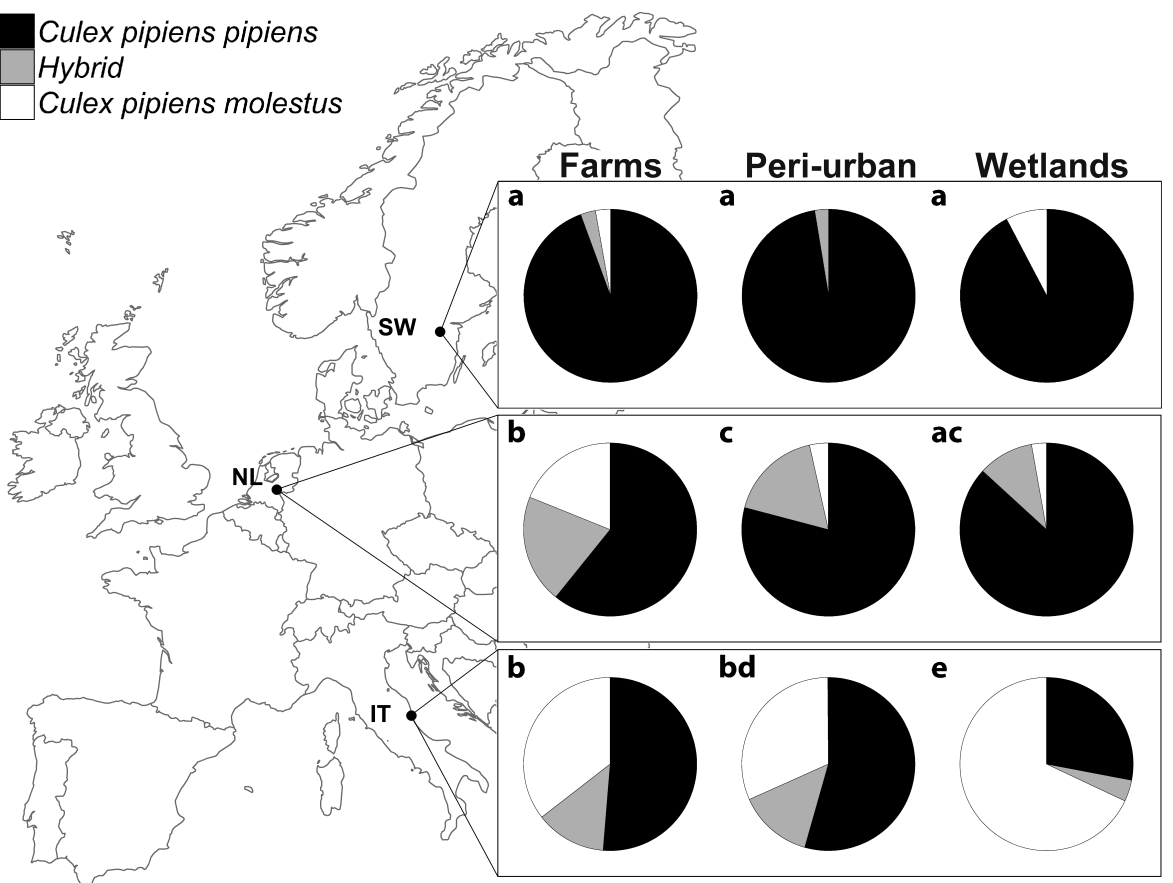

Figure 2: Within-effect of habitat within each of the three countries on the ratio of Culex pipiens biotypes and their hybrids (rows), and within-effect of country within each habitat type (columns). The sample size for each pie chart ranges between $n=26-115$. Letters display significant differences between ratios shown in rows and columns, at a significance level of $p<0.05$. SW $=\mathrm{Sweden}, \mathrm{NL}=\mathrm{The}$ Netherlands, and IT = Italy.

\section{Discussion}

The aim of this study was to assess the relative abundance of the $C x$. pipiens biotypes and their hybrids in different habitats from northern to southern latitudes in Europe, using two trap types. We found a strong latitudinal effect on the ratios of the $C x$. pipiens biotypes and hybrids, with a gradient of decreasing biotype pipiens from northern to southern latitudes. Habitat types also influenced the ratios of $C x$. pipiens biotypes and hybrids, but effects were not consistent at the different latitudes.

Due to low numbers, $C x$. torrentium was excluded from the analyses in this study. All $C x$. torrentium females that we identified originated from Sweden, whereas no $C x$. torrentium females were found in The Netherlands and Italy. These results are consistent with previous studies that showed a relatively high abundance of $C x$. torrentium in northern European countries (Hesson et al. 2011, Hesson et al. 2013), and relatively low abundance or even absence in southern Europe (Di Luca et al. 2016). 
No difference was found between the ratio of $C x$. pipiens biotypes and their hybrids collected with the BGS and MMLP traps. Despite the differences in trapping mechanism between the two traps, there was no apparent difference in the attraction of the biotypes and their hybrids towards both traps. For studies focusing on relative abundance both traps can thus be used equally well, but differences in the total numbers of collected $C x$. pipiens mosquitoes between both traps do exist. Results of studies on mosquito abundance with different traps may not be directly comparable.

Our study shows the sympatric occurrence of both $C x$. pipiens biotypes and hybrids in aboveground habitats throughout Europe. These results are in line with previous findings from The Netherlands (Vogels et al. 2015), Germany (Rudolf et al. 2013), Austria (Zittra et al. 2016), Portugal (Gomes et al. 2009, Osório et al. 2014), Italy (Di Luca et al. 2016), and Greece (Gomes et al. 2013a). There was a clear gradient of decreasing biotype pipiens and increasing biotype molestus proportions from northern to southern latitudes. In Sweden the major part of the $C x$. pipiens mosquitoes collected consisted of the pipiens biotype, whereas ratios in Italy were more equal between pipiens and molestus biotypes. This pattern was visible when ratios were determined per country without differentiating between the different habitat types, as well as when ratios for each country were split over the three habitat types. Previously, only a single record of the molestus biotype was known for Sweden (Schäfer \& Lundström 2001). In addition, the proportion of hybrids in Sweden was much lower compared to The Netherlands and Italy, which can be explained by the near absence of biotype molestus in all habitat types in Sweden. In this study we confirm that both biotypes and their hybrids occur aboveground at latitudes up to $58^{\circ} 24^{\prime} 36^{\prime \prime} \mathrm{N}$.

All peri-urban habitats combined had a relatively higher proportion of biotype pipiens and fewer biotype molestus compared to farms and wetlands. This pattern was, however, not consistent when comparing habitats within each of the three countries. In Sweden the ratios were similar for the three habitats, whereas in The Netherlands farms and in Italy wetlands ratios were different from the other two habitat types. This inconsistency could be explained by differences in, for instance, climate, microhabitat, availability of breeding sites, and hosts which all may influence the presence of the biotypes. These factors are likely to differ more between countries at different latitudes than between nearby habitats at one geographic location. Our findings also show variation at the local scale between habitats in The Netherlands and Italy. Especially the Italian wetlands stand out because of the high proportion of biotype molestus. Although a higher proportion of biotype pipiens was expected in such bird-rich habitats, a previous study showed high proportions of biotype molestus up to $82 \%$ in both urban and rural habitats with aboveground breeding sites in Italy (Di Luca et al. 2016). The same study also showed high variation in Cx. 
pipiens biotype composition throughout Italy (Di Luca et al. 2016). The ratios that we found for each country do, therefore, not represent an overall ratio of $C x$. pipiens for the entire country, but rather for the specific sampling location. The sampling strategy used in our study is suitable for direct comparisons between locations at different latitudes, due to the consistent design over all countries. Studies that place traps at random locations throughout a country are more useful to get insight in local variation and dynamics of Cx. pipiens within a country (Di Luca et al. 2016, Osório et al. 2014, Rudolf et al. 2013, Vogels et al. 2015, Zittra et al. 2016).

$C x$. pipiens populations that are dominated by biotype pipiens play an important role in the natural transmission cycle of WNV in birds, whereas the risk of WNV outbreaks among humans is increased in populations with high levels of hybridization (Fonseca et al. 2004). Up to now, outbreaks of WNV among humans have only occurred in southern and central Europe, including Italy (Rizzoli et al. 2015b). The overall proportion of hybrids is higher in The Netherlands than Italy, which is not consistent with the more equal proportion of biotypes in Italy than in The Netherlands. This indicates a more complex cause of hybridization than solely density dependence. If WNV would get established in The Netherlands, the higher degree of hybridization may result in a higher likelihood of bridging of WNV from birds to humans. However, other factors such as vector competence and climate determine whether transmission cycles can get established. Such factors are most likely limiting the transmission of WNV in northern Europe (Fros et al. 2015a, Vogels et al. 2016a). Future studies on vector competence of the $C x$. pipiens biotypes and hybrids under different climatic scenarios are needed in order to gain more insight in the risk of the transmission of viruses by mosquitoes in Europe (chapters $4 \& 5$ of this thesis).

\section{Conclusions}

The BGS and MMLP traps collected equal ratios of the $C x$. pipiens biotypes and their hybrids. A clear gradient of decreasing biotype pipiens and increasing biotype molestus proportions from northern to southern latitudes in Europe was found. Hybrids were found in all countries, but highest proportions were recorded in The Netherlands and Italy. Furthermore, Cx. pipiens ratios between habitat types were different. These differences were, however, not consistent when comparing habitat types within countries. Future research should focus on, (i) vector competence of $C x$. pipiens biotypes and hybrids at different latitudes in Europe to assess the risks of WNV transmission in northern Europe (chapters $4 \& 5$ of this thesis), and (ii) the ecology of hybrids in order to estimate the risk of WNV being transmitted to humans. 


\section{Acknowledgements}

We would like to thank Claudia Damiani, Luca Bracchetti and Mahmod Hamdan from the Laboratory of Entomology and Parasitology, University of Camerino in Italy, and Rickard Pettersson and Malin Tälle from the department of Theoretical Biology, Linköping University in Sweden for their collaboration and assistance with data collection in the field. We also thank Marcel Dicke for providing comments on a previous version of this chapter. Finally, we thank the participating farmers, nature area managers and home-owners in Sweden, The Netherlands, and Italy that provided us with the essential field locations. 



\section{Chapter 4}

\section{Vector competence of northern European Culex pipiens biotypes and hybrids for West Nile virus is differentially affected by temperature}

Chantal B.F. Vogels ${ }^{1}$, Jelke J. Fros ${ }^{2,3}$, Giel P. Göertz², Gorben P. Pijlman², and Constantianus J.M. Koenraadt ${ }^{1}$

\footnotetext{
${ }^{1}$ Laboratory of Entomology, Wageningen University \& Research

${ }^{2}$ Laboratory of Virology, Wageningen University \& Research

${ }^{3}$ Nuffield Department of Medicine, University of Oxford
}

Parasites \& Vectors (2016) 9: 393 


\begin{abstract}
Outbreaks of West Nile virus (WNV) have not occurred in northern Europe despite nearby circulation of WNV in the southern part of the continent. The main vector for WNV, the mosquito Culex (Cx.) pipiens, consists of two behaviorally distinct biotypes, pipiens and molestus, which can form hybrids. Although temperature has been shown to influence vector competence of $C x$. pipiens for WNV and biotypes are differentially susceptible towards infection, the interaction between the two has not been elucidated. We determined vector competence of the $C x$. pipiens biotypes and hybrids, after 14 days of incubation at $18{ }^{\circ} \mathrm{C}, 23^{\circ} \mathrm{C}$, or $28^{\circ} \mathrm{C}$. Mosquitoes were orally infected by providing an infectious blood meal or by injecting WNV directly in the thorax. Infection and transmission rates were determined by testing the bodies and saliva for WNV presence. In addition, titers of mosquitoes with WNV-positive bodies and saliva samples were determined. Orally infected biotype pipiens and hybrids showed significantly increased transmission rates with higher temperatures, up to $32 \%$ and $14 \%$, respectively. In contrast, the molestus biotype had an overall transmission rate of $10 \%$, which did not increase with temperature. All mosquitoes that were infected via WNV injections had (close to) $100 \%$ infection and transmission rates, suggesting an important role of the mosquito midgut barrier. We found no effect of increasing temperature on viral titers. Temperature differentially affects vector competence of the $C x$. pipiens biotypes. This shows the importance of accounting for biotype-by-temperature interactions, which influence the outcomes of vector competence studies. Vector competence studies with $C x$. pipiens mosquitoes differentiated to the biotype level are essential for proper WNV risk assessments.
\end{abstract}

Keywords: arbovirus, Culex, vector competence, West Nile virus, infection, temperature 


\section{Introduction}

West Nile virus (WNV; family: Flaviviridae) is the most widespread arthropodborne (arbo)virus in the world and can be fatal for humans (Kramer et al. 2008). The enzootic cycle of WNV is maintained among mosquitoes and birds, whereas mammals (including humans) are dead-end hosts. Outbreaks of WNV have not occurred in northern Europe despite the high abundance of competent mosquito vectors (Fros et al. 2015a, Fros et al. 2015b), susceptible bird hosts (Lim et al. 2015, Lim et al. 2014), and nearby circulation of WNV in southern Europe (Hubalek \& Halouzka 1999).

The epidemic potential of WNV is best illustrated by the repeated outbreaks in humans in North America (Hayes et al. 2005). After the initial introduction of WNV in New York in 1999, WNV rapidly spread across the continent, resulting in the largest outbreak of neuroinvasive disease to date (Hayes \& Gubler 2006, Lanciotti et al. 1999). American passeriform bird species are the main hosts for WNV, with the family of the corvids (family: Corvidae) being highly susceptible (Hayes \& Gubler 2006, Komar et al. 2003, Wheeler et al. 2009, Work et al. 1955). Compared to North America, WNV-associated death rates among birds in southern Europe have been relatively low. It has, therefore, been hypothesized that European bird species are less susceptible to WNV compared to their North American counterparts. Recent studies confirmed, however, that three important bird species originating from Europe, the carrion crow, European jackdaw, and house sparrow, are highly susceptible to WNV (Del Amo et al. 2014, Lim et al. 2015, Lim et al. 2014).

Culex (Cx.) pipiens mosquitoes have been identified as the most important vector species for WNV in the United States, because of their vector competence and high abundance during summer (Kilpatrick et al. 2005). The species Cx. pipiens consists of two morphologically identical biotypes, named pipiens (Linnaeus 1758) and molestus (Forskål 1775), which show distinct behavior (Byrne \& Nichols 1999, Vinogradova 2000). Biotype pipiens prefers birds as blood hosts whereas biotype molestus prefers mammals (Byrne \& Nichols 1999). As a consequence, biotype pipiens plays an important role in the natural transmission cycle of WNV. Hybrids between the two biotypes have an intermediate host preference, which makes them ideal vectors to bridge WNV from birds to mammals (Fritz et al. 2015). The presence and abundance of hybrids may, therefore, strongly increase the risk of WNV outbreaks in the human population.

Previous studies showed that proper identification and separation of biotypes is essential, because genetic differences between biotypes and populations influence vector competence (Brustolin et al. 2016, Ciota et al. 2013a, Nelms et al. 2013). Furthermore, our previous studies showed that temperature is an important 
determinant of vector competence of $C x$. pipiens mosquitoes for WNV (Fros et al. 2015a, Fros et al. 2015b). However, vector competence of the Cx. pipiens biotypes has not been tested at different temperatures. Transmission of another arbovirus, chikungunya virus, has been shown to strongly depend on complex interactions between Aedes albopictus populations, viral strains, and temperature (Zouache et al. 2014). The aim of this study was, therefore, to determine the effect of the interaction between temperature and the $C x$. pipiens biotypes and their hybrids on WNV transmission. Vector competence studies with northern European populations of both $C x$. pipiens biotypes and hybrids at different temperatures are necessary, to assess the risks for northern Europe in the light of climate change (Beniston et al. 2007, Meehl \& Tebaldi 2004).

\section{Materials and Methods}

\section{Mosquitoes}

Culex pipiens egg rafts were collected during June and July 2014 from aboveground water barrels in Best, The Netherlands. One larva from each egg raft was identified to the biotype level with real-time PCR (Vogels et al. 2015). A pipiens colony was started by grouping larvae from 162 egg rafts molecularly identified as biotype pipiens. F3 offspring were used for oral virus infection studies, and F5 offspring for virus infection via intra-thoracic injections. Culex pipiens biotype molestus egg rafts and larvae were collected during September 2013 and February 2014 from an underground habitat in Schiphol airport, Amsterdam, The Netherlands. The colony was started from 38 egg rafts that were autogenously laid by the mosquitoes that emerged from the egg rafts and larvae collected at Schiphol. Real-time PCR confirmed the molestus biotype. Crosses between male biotype pipiens and female biotype molestus resulted in F1 hybrid progeny. The reverse cross between biotypes was not able to produce viable offspring.

All larvae and adults were maintained at $23^{\circ} \mathrm{C}$ with $16: 8$ light:dark cycle and $60 \%$ relative humidity. Egg rafts were transferred to trays $(25 \mathrm{~cm} \times 25 \mathrm{~cm} \times 8 \mathrm{~cm})$ filled with water and a drop of Liquifry No. 1 (Interpet Ltd., UK). Thereafter, they were daily fed with a 1:1:1 mixture of bovine liver powder, ground rabbit food and ground koi food. Pupae were transferred to Bugdorm cages $(30 \mathrm{~cm} \times 30 \mathrm{~cm} \times 30 \mathrm{~cm})$ and provided with $6 \%$ ad libitum glucose solution. Bovine or chicken blood (Kemperkip, The Netherlands) was provided through a Hemotek PS5 feeder (Discovery Workshops, UK) for egg production. Female mosquitoes were kept together with males for 4 to 19 days before being transferred to the Biological Safety Level (BSL) 3 facility. Variation in age was kept similar for the two biotypes and their hybrids. To increase mosquito blood-feeding rates, glucose solution was replaced by water 4 to 6 days 
before the infectious blood meal was offered.

\section{Virus}

In all experiments a P2 stock of West Nile virus lineage 2 originating from Greece (2010) was used with a $50 \%$ tissue culture infective dose ranging between $7.96 \times$ $10^{7} \mathrm{TCID}_{50} / \mathrm{ml}$ and $1.12 \times 10^{9} \mathrm{TCID}_{50} / \mathrm{ml}$. WNV was grown on Aedes albopictus C6/36 cells and titrated on green monkey kidney Vero E6 cells. C6/36 cells were cultured with Leibovitz L15 medium (Life technologies, The Netherlands) supplemented with $10 \%$ fetal bovine serum (FBS; Life technologies). Vero E6 cells were cultured with hepes-buffered DMEM medium (Life technologies) supplemented with $10 \%$ FBS, penicillin (100 IU/ml; Sigma-Aldrich, The Netherlands) and streptomycin $(100 \mu \mathrm{g} /$ $\mathrm{ml}$; Sigma-Aldrich). The medium was fully supplemented by adding fungizone (2,5 $\mu \mathrm{g} / \mathrm{ml}$; Life technologies) and gentamycin ( $50 \mu \mathrm{g} / \mathrm{ml}$; Life technologies) when viral infection or titers were determined from mosquito homogenates.

\section{West Nile virus infections}

To determine the period until peak transmission rates and viral titers have developed, we determined viral titers up to 35 days post infection. Biotype pipiens mosquitoes were injected with $69 \mathrm{~nL}$ of WNV $\left(1.12 \times 10^{9} \mathrm{TCID}_{50} / \mathrm{ml}\right)$ using the Drummond Nanoject II Auto-Nanoliter Injector (Drummond Scientific Company, USA). Capillaries (3.5" Drummond \# 3-000-203-G/X, Drummond Scientific Company) were drawn to a fine point with the Narishige needle puller model PB-7 (Narishige, Japan). Injections instead of infectious blood meals were used in order to obtain $100 \%$ infection (Fros et al. 2015a). Injected mosquitoes were divided over 6 groups of 24 female mosquitoes, and placed in buckets $(\varnothing: 12.5 \mathrm{~cm}, \mathrm{~h}: 12.5 \mathrm{~cm})$ at $23^{\circ} \mathrm{C}$ for $7,14,21$, 28 , or 35 days. One group of female mosquitoes was directly killed (day 0 ) and individually stored at $-80^{\circ} \mathrm{C}$.

The $C x$. pipiens biotypes and hybrids were orally infected with WNV in order to test for the effect of temperature on infection and transmission rates (Fros et al. 2015a). In the BSL3 facility, biotype pipiens, biotype molestus, and hybrid mosquitoes were kept in groups of 20-100 females in buckets. Female mosquitoes were allowed to feed for one hour on infectious chicken blood with a titer of $5.7 \pm 1.0 \times 10^{7} \mathrm{TCID}_{50} /$ $\mathrm{ml}$, offered through a Hemotek PS5 feeder. The blood meal was offered during the early stage of the dark period in a dark room at $24{ }^{\circ} \mathrm{C}$ and $60 \% \mathrm{RH}$. After 1 hour, mosquitoes were immobilized on a semi-permeable $\mathrm{CO}_{2}$-pad connected to $100 \%$ $\mathrm{CO}_{2}$. Blood fed mosquitoes of each group were selected and divided in smaller groups of maximum 20 female mosquitoes over three separate buckets. The buckets were placed at $18{ }^{\circ} \mathrm{C}, 23^{\circ} \mathrm{C}$, or $28^{\circ} \mathrm{C}$ for 14 days. Oral infections were replicated four to seven times until a total number of 50 female mosquitoes of each biotype per 
temperature was reached.

To determine the effect of the midgut on WNV dissemination, mosquitoes were also injected with WNV. Biotype pipiens, biotype molestus, and hybrid mosquitoes were immobilized with $\mathrm{CO}_{2}$, and injected in the thorax with $69 \mathrm{~nL}$ of WNV $\left(1.12 \times 10^{9}\right.$ $\mathrm{TCID}_{50} / \mathrm{ml}$ ). Mosquitoes from each group were divided in groups of maximally 12 female mosquitoes over three separate buckets and placed at $18{ }^{\circ} \mathrm{C}, 23{ }^{\circ} \mathrm{C}$, or 28 ${ }^{\circ} \mathrm{C}$ for 14 days. Injections were replicated three times until a total number of $20 \pm 1$ female mosquitoes of each biotype per temperature was reached.

\section{Infection and transmission rates}

After the incubation period of 14 days, mosquitoes were immobilized with $\mathrm{CO}_{2}$ and legs and wings were removed. To collect saliva, the proboscis of each mosquito was inserted in a pipette tip with $5 \mu \mathrm{l}$ of a $1: 1$ solution of FBS and $50 \%$ glucose solution, for a minimum period of 45 minutes. Each saliva sample was collected in a $1.5 \mathrm{ml}$ SafeSeal micro tube with $55 \mu \mathrm{l}$ of fully supplemented hepes-buffered DMEM medium, whereas bodies were individually stored in $1.5 \mathrm{ml}$ SafeSeal micro tubes with a small scoop of $0.5 \mathrm{~mm}$ Zirconium beads (Bio-Connect BV, The Netherlands). All samples were stored at $-80^{\circ} \mathrm{C}$ until further use.

Frozen mosquito bodies were homogenized in the bullet blender storm (Next Advance, USA) for 2 minutes, and shortly spun down at 14,000 rpm. Next, 100 $\mu \mathrm{l}$ of fully supplemented hepes-buffered DMEM medium was added and samples were again homogenized for 2 minutes in the bullet blender, and spun down for 2 minutes at 14,000 rpm. For each mosquito homogenate or saliva sample, $30 \mu \mathrm{l}$ was incubated on a monolayer of Vero E6 cells in a 96 wells plate. After 2-3 hours the medium was completely removed and replaced by $100 \mu$ of new medium. After three days, each well was scored for cytopathic effects specific for WNV. Titers were determined by endpoint dilution assays on Vero E6 cells, and scored for cytopathic effects after 3 days.

\section{Statistical analysis}

Generalized linear models with a binomial distribution and logit link function were used to test for the effects of biotype and temperature on the WNV infection and transmission rates, for the orally infected mosquitoes. Mosquitoes with positive saliva, but negative body (1\%) were excluded from the analysis. Infection and transmission rates were calculated, respectively, by dividing the number of female mosquitoes with infected bodies or with infected saliva by the total number of female mosquitoes in the respective treatment. Effects of replicate, biotype, temperature, and the interaction term between biotype and temperature, were included in both 
models. When interaction terms were significant, the effect of temperature was tested separately for each biotype with likelihood ratio tests. Multiple comparisons were corrected with the Bonferroni procedure, by lowering the significance level from 0.05 to 0.017 . The effect of temperature on viral titers was tested separately for each biotype with Wilcoxon or Kruskal-Wallis tests. All statistical analyses were done with SAS software, version 9.3 (SAS Institute Inc., USA), and figures were created with the statistical software package $R$ (R Core Team 2016).

\section{Results}

To determine the minimum incubation period until peak viral titers were reached, viral titers were assessed for $C x$. pipiens biotype pipiens females up to 35 days post WNV infection. Infection rates were $100 \%$ at all time points from day zero to 35 post infection (Figure 1A). In addition, at all time points all mosquitoes tested had viruspositive saliva except for one female at day 21 (Figure 1A). After 14 days, viral titers of the mosquito bodies were approximately 1000-fold higher than titers at the day of infection, and remained high over time (average titer of $2 \times 10^{7} \mathrm{TCID}_{50} / \mathrm{ml}$; Figure 1B).
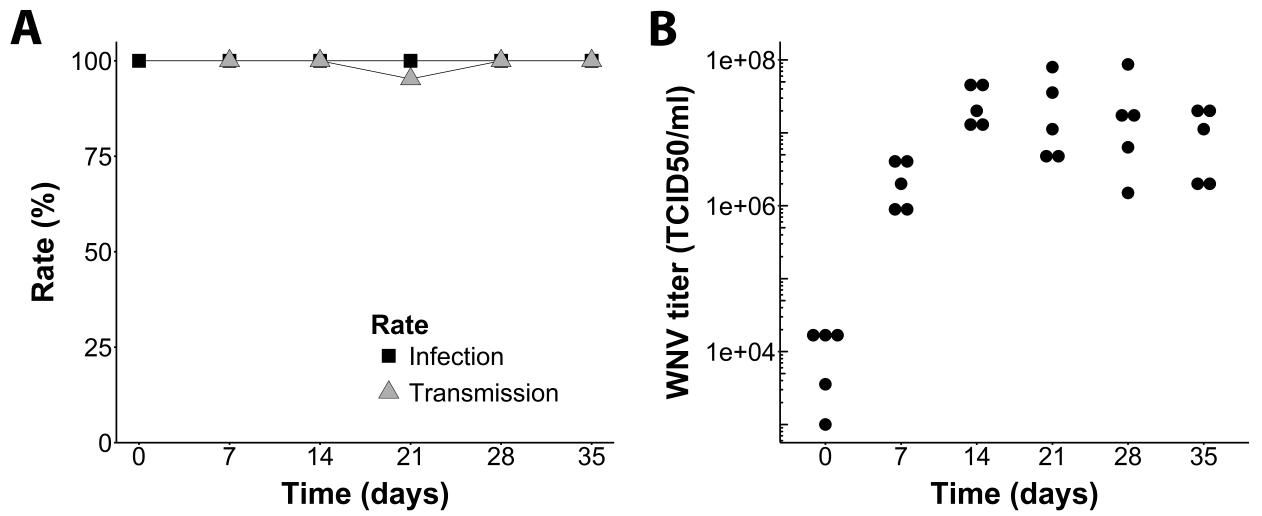

Figure 1: Vector competence and titers of West Nile virus injected Culex pipiens biotype pipiens females over 35 days. (A) Infection (squares) and transmission (triangles) rates of biotype pipiens females incubated at $23^{\circ} \mathrm{C}$ from day 0 to 35 post injection. Each data point represents $16-24$ mosquitoes. (B) West Nile virus titers of five selected female mosquitoes at each time point from day 0 to 35 post infection.

Infection and transmission rates were determined for orally infected biotype pipiens, biotype molestus, and hybrid females after 14 days of incubation at $18^{\circ} \mathrm{C}, 23^{\circ} \mathrm{C}$ or $28^{\circ} \mathrm{C}$. Initial model analyses of infection rates showed a significant interaction effect between biotype and temperature (GLM, $X^{2}=12.89, P=0.012$ ), which indicated 
that infection rates of biotypes responded differently to temperature (Figure 2A). Selections were, therefore, made to test for the effect of temperature separately for each biotype. Infection rates were significantly different between temperatures for biotype pipiens (28.6-63.3 \%; Likelihood ratio test (LR), $X^{2}=12.54, P=0.002$ ), but there was no significant difference for biotype molestus (24.5-14.0\%; LR, $X^{2}=3.96$, $P=0.14)$, and hybrids (24.0-42.9\%; LR, $\left.X^{2}=5.07, P=0.080\right)$. For biotype pipiens, the infection rate was significantly higher at $28^{\circ} \mathrm{C}$ compared to $18^{\circ} \mathrm{C}\left(\mathrm{LR}, \mathrm{X}^{2}=12.31\right.$, $P<0.001)$, but not at $23^{\circ} \mathrm{C}$ compared to $18^{\circ} \mathrm{C}\left(\mathrm{LR}, X^{2}=4.91, P=0.027\right.$ (threshold $P$-value $=0.017$ after Bonferroni correction)), and $23{ }^{\circ} \mathrm{C}$ compared to $28{ }^{\circ} \mathrm{C}(\mathrm{LR}$, $\left.\mathrm{X}^{2}=1.79, P=0.18\right)$. Thus, infection rates of biotype pipiens increased with higher temperatures, with the strongest increase at $28^{\circ} \mathrm{C}$, whereas there was no effect of temperature on infection rates of biotype molestus and hybrids.

To determine whether a female mosquito can actually transmit WNV, transmission rates were determined based on the presence of WNV in the saliva. Model analyses again showed a significant interaction effect between biotype and temperature (GLM, $X^{2}=15.71, P=0.003$ ), which indicated that also transmission rates of the biotypes responded differentially to temperature (Figure 2B). Therefore, the effect of temperature on the transmission rate of each biotype was tested separately. Transmission rates significantly increased with higher temperatures for biotype pipiens (0-32.7 \%; LR, $\left.X^{2}=29.95, P<0.001\right)$, and hybrids $\left(0-14.3 \% ; L R, X^{2}=13.49\right.$, $P=0.001)$, but not for biotype molestus (6.1-10.2 \%; LR, $\left.X^{2}=0.77, P=0.68\right)$. For biotype pipiens, the transmission rate was significantly higher at $28{ }^{\circ} \mathrm{C}$ compared to $18{ }^{\circ} \mathrm{C}\left(\mathrm{LR}, \mathrm{X}^{2}=26.41, P<0.001\right)$ and compared to $23^{\circ} \mathrm{C}\left(\mathrm{LR}, \mathrm{X}^{2}=12.75, P<\right.$ $0.001)$, but not at $23{ }^{\circ} \mathrm{C}$ compared to $18{ }^{\circ} \mathrm{C}\left(\mathrm{LR}, \mathrm{X}^{2}=4.29, P=0.038\right.$ (threshold $P$-value $=0.017$ after Bonferroni correction)). Also for hybrids, the transmission rate was significantly higher at $28{ }^{\circ} \mathrm{C}$ compared to $18{ }^{\circ} \mathrm{C}\left(\mathrm{LR}, X^{2}=6.58, P=0.010\right)$ and compared to $23^{\circ} \mathrm{C}\left(\mathrm{LR}, \mathrm{X}^{2}=11.18, P<0.001\right)$, but there was no difference between $18{ }^{\circ} \mathrm{C}$ and $23{ }^{\circ} \mathrm{C}\left(\mathrm{LR}, \mathrm{X}^{2}=1.35, P=0.25\right)$. Thus, transmission rates of biotypes responded differently to temperature, with biotype pipiens and hybrids showing a significant increase with higher temperature, whereas there was no effect of temperature on biotype molestus.

In order to test whether different responses of biotypes to temperature could be attributed to the midgut barrier or the salivary gland barrier, the $C x$. pipiens biotypes and hybrids were injected with WNV. By injecting WNV directly in the thorax the midgut barrier is bypassed, and only the salivary gland barrier needs to be overcome before WNV can be transmitted. After 14 days of incubation, the infection rates were $100 \%$ for both biotypes and hybrids, at all temperatures (Figure 2A). Transmission rates of female mosquitoes at most temperatures were (close to) $100 \%$ (Figure 2B), suggesting that there is no apparent salivary gland barrier. 

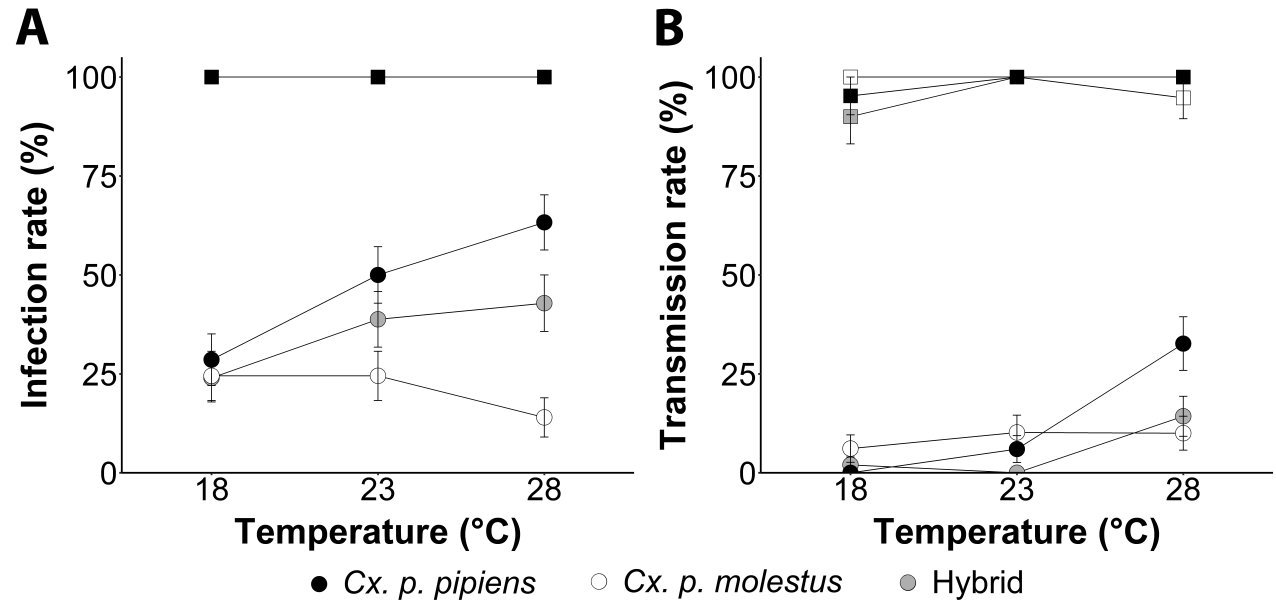

Figure 2: Vector competence of the Culex pipiens biotypes and hybrids incubated at $18^{\circ} \mathrm{C}, 23^{\circ} \mathrm{C}$, or $28^{\circ} \mathrm{C}$ for 14 days. (A) Infection rates and (B) transmission rates of biotype pipiens (black), biotype molestus (white), and hybrids (grey) after oral infection (circles) and injections (squares). Data points of the biotypes and hybrids after injections are overlapping. Each data point represents 50 female mosquitoes for oral infections, and $20 \pm 1$ female mosquitoes for injections. Error bars show standard error of the means.

Finally, viral titers of all orally infected female mosquitoes with virus-positive body and saliva were determined. The effect of temperature on viral titer was again tested separately for each biotype. There was no significant effect of temperature on median viral titers of biotype pipiens (Wilcoxon, $Z=1.51, P=0.13$; Figure $3 \mathrm{~A}$ ), biotype molestus (KW, $\mathrm{X}^{2}=1.96, P=0.38$; Figure $3 \mathrm{~B}$ ), and hybrids (Wilcoxon, $\mathrm{Z}=1.40, P$ $=0.13$; Figure $3 \mathrm{C}$ ). These results demonstrate that viral titers did not increase with higher temperatures, indicating that after successfully passing the midgut barrier there is no difference between the biotypes.
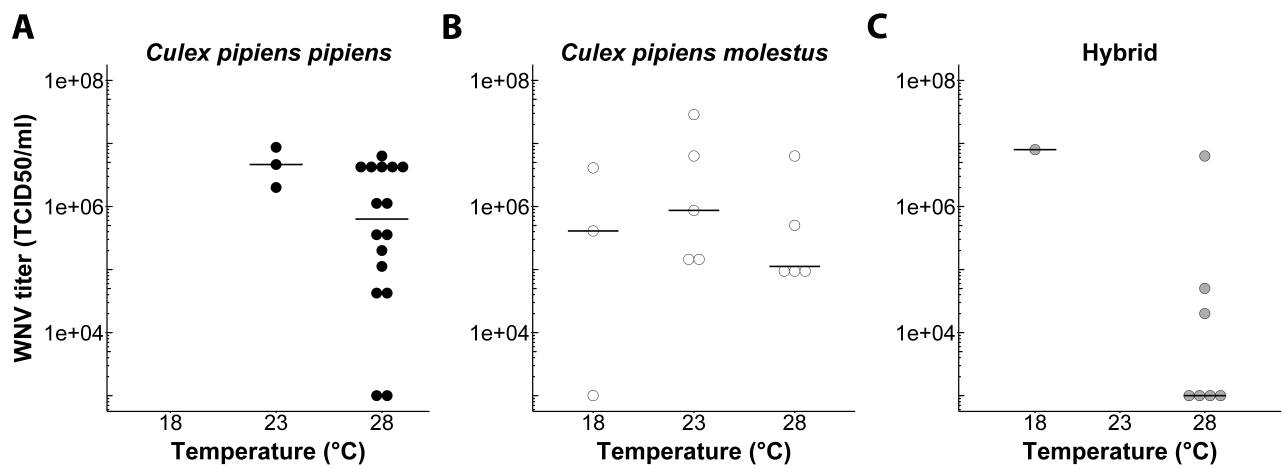

Figure 3: West Nile virus titers of orally infected Culex pipiens females with virus-positive bodies and saliva. West Nile virus titers were determined after 14 days of incubation at $18{ }^{\circ} \mathrm{C}, 23^{\circ} \mathrm{C}$, or $28^{\circ} \mathrm{C}$ for $(A)$ biotype pipiens $(B)$ biotype molestus and $(C)$ hybrids. Horizontal black lines show median titers at each temperature. 


\section{Discussion}

The aim of this study was to investigate the interaction between temperature and $C x$. pipiens biotypes on WNV transmission. We found that temperature differentially affected vector competence of the $C x$. pipiens biotypes and their hybrids. Transmission rates of biotype pipiens (0-33 \%) and hybrids (0-14\%) increased with higher temperature, especially at $28{ }^{\circ} \mathrm{C}$, whereas transmission rates of biotype molestus (6-10\%) were not affected by temperature. Transmission rates of biotype pipiens were within the range of other $C x$. pipiens populations in WNV endemic areas (Balenghien et al. 2008, Fortuna et al. 2015a, Kilpatrick et al. 2008). Peak transmission rates of biotype molestus and hybrids were, however, two- to three-fold lower.

Positive relations between temperature and vector competence are not limited to WNV and Cx. pipiens (Fros et al. 2015a, Kilpatrick et al. 2008), but have also been described for other virus-vector systems (Fros et al. 2015b, Zouache et al. 2014). This positive relation is most likely the result of increased replication of the virus at higher temperatures (Chamberlain \& Sudia 1961, Fros et al. 2015b, Hardy et al. 1983). Interestingly, the transmission rates of biotype molestus did not increase with higher temperature. In this study, all female mosquitoes were reared under the same controlled conditions. Observed differences between biotypes are, therefore, likely due to interactions between genotype and temperature. When bypassing the midgut barrier by injecting WNV in female mosquitoes, transmission rates were similar for the biotypes and their hybrids. This suggests that genetic factors determining midgut characteristics can explain why temperature had no effect on transmission rates of biotype molestus. The highest temperature may have induced changes in the morphology and physiology of the midgut of biotype pipiens and hybrids. As a result, the effectiveness of the midgut as a barrier may have decreased, whereas there seemed to be a higher threshold for biotype molestus. Such lowered effectiveness of the midgut barrier at higher temperatures has been shown with Aedes albopictus mosquitoes infected with dengue virus (Alto \& Bettinardi 2013). Alternatively, temperature may have induced changes in the regulation of biotype-specific immunoresponsive genes, as has been shown for Aedes aegypti mosquitoes infected with chikungunya virus (Mourya et al. 2004). In addition, the RNAi pathway becomes more active at higher temperatures and can inhibit viral replication (Adelman et al. 2013). Differences in RNAi activity between the biotypes may have resulted in increased sensitivity for WNV infection of biotype pipiens and hybrids at higher temperatures, whereas such changes were not induced in biotype molestus. Future studies should focus on the underlying mechanisms in the midgut that can explain why vector competence of biotype molestus for WNV did not increase with higher temperature. 
Vector competence of $C x$. pipiens for WNV varies in time and among different mosquito populations (Vaidyanathan \& Scott 2007). Vector competence of North American lines of biotype pipiens (Pennsylvania) and of biotype molestus (New Jersey) populations were equal, but lower than vector competence of their hybrid progeny (Ciota et al. 2013a). In contrast, significant differences in vector competence were found between biotype pipiens (Woodland) and biotype molestus (Sacramento) populations (Nelms et al. 2013). No differences in transmission rates were found between four different autogenous and anautogenous $C x$. pipiens populations in Italy (Fortuna et al. 2015a). Our study shows that such variation can be partly explained by temperature, but other complex interactions between viral strains, viral titers, and geographically distinct vector populations have been shown to play a role as well (Anderson et al. 2010, Fros et al. 2015a, Zouache et al. 2014). In addition, colonization in the laboratory could have influenced the outcomes of these vector competence studies (Gargan et al. 1983, Grimstad et al. 1977, Jones \& Foster 1978, Lorenz et al. 1984). Such effects have been shown to occur after 8 generations for Cx. pipiens mosquitoes infected with Rift Valley Fever virus (Gargan et al. 1983), but this is dependent on the number of females the colony was established with, and selection pressures in the rearing environment (Grimstad et al. 1977, Jones \& Foster 1978). In order to fully understand factors that are required for WNV transmission, a direct comparison should be made between vector competence of recently established mosquito populations originating from endemic and non-endemic areas at different temperatures (chapter 5 of this thesis).

Vector competence is only one qualitative component of the more complex, quantitative vectorial capacity of a species to transmit pathogens (Garrett-Jones 1964a, Garrett-Jones 1964b). Vectorial capacity also takes into account other factors such as vector and host abundance, host preference, biting rate, and survival. Our results confirm that temperature is an important factor that may explain the current absence of WNV transmission in northern Europe. Temperature is, however, not the only factor that can predict when and where WNV transmission cycles can be established (Marcantonio et al. 2015). In order to determine the risk of WNV establishment and further transmission, it is essential to use valid predictors of all components of vectorial capacity in risk models. Our results emphasize the need to characterize $C x$. pipiens populations to the biotype level for reliable assessments of the risk for future WNV outbreaks.

Based on our findings that there is an increased likelihood of WNV transmission by $C x$. pipiens at higher temperatures, and climate change predictions of longer and more intense heat waves during summer (Beniston et al. 2007, Meehl \& Tebaldi 2004), future WNV transmission in northern Europe cannot be ruled out. Preparing for future WNV transmission in northern Europe is, therefore, advised. 


\section{Conclusions}

Vector competence of the $C x$. pipiens biotypes and their hybrids for WNV is differentially affected by temperature. Transmission rates of biotype pipiens and hybrids increased with higher temperatures, but not so for biotype molestus. Our study emphasizes the need to identify $C x$. pipiens mosquitoes to the biotype level because of the differential vector competence and thus vectorial capacity for WNV.

\section{Acknowledgements}

We thank Wietse den Hartog for collecting biotype molestus from the field, Corinne Geertsema for maintenance of cell cultures, Gerrit Gort for support with statistical analyses, and Marcel Dicke for providing comments on an earlier version of this chapter. 



\section{Chapter 5}

\section{Vector competence of northern and southern European Culex pipiens pipiens mosquitoes for West Nile virus across a gradient of temperatures}

Chantal B.F. Vogels ${ }^{1}$, Giel P. Göertz², Gorben P. Pijlman², and Constantianus J.M. Koenraadt ${ }^{1}$

${ }^{1}$ Laboratory of Entomology, Wageningen University \& Research

${ }^{2}$ Laboratory of Virology, Wageningen University \& Research

Medical and Veterinary Entomology (in press) 


\begin{abstract}
In Europe, West Nile virus (WNV) outbreaks have been limited to southern and central European countries. However, competent mosquito vectors and susceptible bird hosts are present in northern Europe. Differences in temperature and vector competence of mosquito populations may explain the absence of WNV outbreaks in northern Europe. The aim of this study was to directly compare vector competence of northern and southern European Culex pipiens (Cx. p.) pipiens mosquitoes for WNV across a gradient of temperatures. WNV infection and transmission rates were determined for two $C x$. p. pipiens populations originating from The Netherlands and Italy, respectively. Mosquitoes were orally exposed by providing an infectious blood meal, or by injecting WNV (lineage 2 ) in the thorax, followed by 14 days incubation at $18{ }^{\circ} \mathrm{C}, 23{ }^{\circ} \mathrm{C}$, or $28{ }^{\circ} \mathrm{C}$. We found no differences in infection or transmission rates between the $C x$. p. pipiens populations with both infection methods, but WNV transmission rates were significantly higher at temperatures above $18^{\circ} \mathrm{C}$. Absence of WNV outbreaks in northern Europe cannot be explained by differences in vector competence between $C x$. p. pipiens populations originating from northern and southern Europe. This study suggests that low temperature is a key limiting factor for WNV transmission.
\end{abstract}

Keywords: Culex pipiens complex, northern house mosquito, arbovirus, infection, transmission, The Netherlands, Italy 


\section{Introduction}

In Europe, outbreaks of the mosquito-borne West Nile virus (WNV; family: Flaviviridae) among humans and horses are remarkable, because they have been limited to southern and central European countries (Calistri et al. 2010a). This is in clear contrast with the ongoing outbreaks throughout North America since its emergence in 1999 (Hayes et al. 2005). Thus far, no outbreaks have occurred in northern Europe, despite serological evidence of WNV circulation in birds in Germany and the United Kingdom (Buckley et al. 2006, Linke et al. 2007).

WNV is transmitted among susceptible bird hosts by mosquitoes, whereas mammals develop lower viraemia and hence are considered dead-end hosts (Kramer et al. 2008). In North America, Culex (Cx.) pipiens (Diptera, Culicidae) mosquitoes have been identified as one of the main vector species for WNV transmission, due to their vector competence and high abundance (Kilpatrick et al. 2005). The species Cx. pipiens consists of two morphologically identical biotypes, pipiens (Linnaeus 1758) and molestus (Forskål 1775), which differ in physiology and behaviour (Byrne \& Nichols 1999). Particularly the pipiens biotype is an important enzootic vector because of its feeding preference for birds (Fritz et al. 2015). Corvid birds (family: Corvidae) are highly susceptible for WNV infection (Wheeler et al. 2009). Differences in WNV transmission between northern and southern Europe may be explained by lower susceptibility of northern European bird species or lower vector competence of $C x$. pipiens populations, which may be driven by lower temperatures. Recent studies showed that carrion crows (Corvus corone), and European jackdaws (Corvus monedula) originating from The Netherlands are susceptible for infection with different WNV isolates, with viraemia in crows that could support mosquito infection (Lim et al. 2015, Lim et al. 2014). Thus, it is unlikely that low bird susceptibility can explain the lack of WNV transmission in northern Europe.

Culex pipiens mosquitoes originating from The Netherlands and Germany have been found to be competent vectors for WNV (Fros et al. 2015a, Leggewie et al. 2016, Vogels et al. 2016a). However, studies in both Europe and North America have not only shown spatial and temporal variation in vector competence among different mosquito populations (Kilpatrick et al. 2010, Leggewie et al. 2016, Vaidyanathan \& Scott 2007), but also variation in vector competence due to environmental factors (Fros et al. 2015a, Kilpatrick et al. 2008, Vogels et al. 2016a). This suggests that both genetic and environmental factors play an important role in vector competence, which may explain the differences in WNV transmission across Europe.

Thus far, only few studies have determined the vector competence of southern European Cx. pipiens mosquitoes for WNV (Brustolin et al. 2016, Fortuna et al. 2015a). Furthermore, differences in experimental designs hamper the ability to make 
direct comparisons between vector competence studies on $\mathrm{Cx}$. pipiens populations originating from different locations in Europe. The aim of this study was, therefore, to directly compare vector competence of northern (The Netherlands) and southern (Italy) European Cx. pipiens (p.) pipiens mosquitoes for WNV, across a gradient of temperatures.

\section{Materials and Methods}

\section{Mosquitoes}

During June and July 2015, Culex egg rafts were collected from aboveground water barrels in Best, in the south of The Netherlands, and from buckets infused with hay and yeast in San Benedetto del Tronto, at the east coast in central Italy. Egg rafts were placed individually in tubes in which larvae could hatch. Approximately 10 larvae from each egg raft were pooled and identified to the biotype level with real-time PCR (Vogels et al. 2015). The Dutch rearing was started by grouping larvae from 120 egg rafts identified as $C x . p$. pipiens, and the Italian rearing was started by grouping larvae from 114 egg rafts identified as Cx. p. pipiens. The F4-6 offspring of both populations were used in experiments. Mosquitoes were reared as described before (Vogels et al. 2016a). In short, larvae and adults from both colonies were maintained at $23{ }^{\circ} \mathrm{C}$ with a $16: 8 \mathrm{~L}: \mathrm{D}$ cycle and $60 \%$ relative humidity. Larvae were initially fed with Liquifry No. 1 (Interpet Ltd., UK) and after 2 days a 1:1:1 mixture of bovine liver powder (MP biomedicals, USA), ground rabbit food (Pets Place, The Netherlands), and ground koi food (Tetra, Germany) was provided. Adults were provided with 6 $\%$ glucose solution ad libitum. Bovine (Carus, The Netherlands) or chicken blood (Kemperkip, The Netherlands) was provided through Parafilm using the Hemotek PS5 feeder (Discovery Workshops, UK), for egg production. For both mosquito populations, high reproduction rates were observed for all mosquito generations.

Female mosquitoes were kept together with males for 5 to 18 days in Bugdorm-1 insect rearing cages (30 cm x $30 \mathrm{~cm} \times 30 \mathrm{~cm}$; Bugdorm, China), before females were transferred in buckets $(\varnothing: 12.2 \mathrm{~cm}$, height: $12.2 \mathrm{~cm}$; Jokey, Germany) to the Biological Safety Level (BSL) 3 facility. The age range of female mosquitoes from both The Netherlands and Italy was kept similar. Glucose solution was replaced by water 3 to 4 days before the infectious blood meal was offered, in order to stimulate blood feeding.

\section{Virus}

In all experiments a passage 2 stock of WNV lineage 2 (provided by Erasmus Medical Center, The Netherlands; GenBank accession no. HQ537483.1) originating 
from Greece (2010) was used, which was originally isolated from a pool of $C x$. pipiens mosquitoes (Papa et al. 2011). The WNV stock had a titer of $9.2 \pm 0.9 \times$ $10^{7} \mathrm{TCID}_{50} / \mathrm{ml}$. WNV was grown on C6/36 cells and titrated on Vero E6 cells. C6/36 cells were cultured with Leibovitz L-15 medium (Life technologies, The Netherlands) supplemented with $10 \%$ fetal bovine serum (FBS; Life technologies), $2 \%$ tryptose phosphate broth (Life technologies) and $1 \%$ nonessential amino acids (Life technologies), at $28{ }^{\circ} \mathrm{C}$. Vero E6 cells were cultured with hepes-buffered DMEM medium (Life technologies) supplemented with $10 \% \mathrm{FBS}$, penicillin $(100 \mathrm{IU} / \mathrm{ml}$; Sigma-Aldrich, The Netherlands) and streptomycin (100 $\mu \mathrm{g} / \mathrm{ml}$; Sigma-Aldrich), at $37{ }^{\circ} \mathrm{C}$. The medium was fully supplemented by adding fungizone $(2.5 \mu \mathrm{g} / \mathrm{ml}$; Life technologies) and gentamycin (50 $\mu \mathrm{g} / \mathrm{ml}$; Life technologies) when samples were tested for presence of virus.

\section{West Nile virus infections}

In this study, the same protocols were used as described before (Vogels et al. 2016a). In order to test for the effect of population and temperature on WNV infection and transmission rates, mosquitoes were orally exposed to WNV. Culex p. pipiens mosquitoes originating from The Netherlands and Italy were allowed to feed for 1 hour on infectious chicken blood with a titer of $4.2 \times 10^{7} \mathrm{TCID}_{50} / \mathrm{ml}$, offered through a Parafilm membrane using the Hemotek PS5 feeder in the BSL3 facility. The blood meal was offered during the early stage of the dark period in a dark room at $24^{\circ} \mathrm{C}$ and $60 \% \mathrm{RH}$. After 1 hour, mosquitoes were anaesthetized with $\mathrm{CO}_{2}$ and immobilized on a semi-permeable $\mathrm{CO}_{2}$-pad connected to $100 \% \mathrm{CO}_{2}$. Engorged females of each group were selected and divided over three separate buckets. The buckets were placed at $18{ }^{\circ} \mathrm{C}, 23{ }^{\circ} \mathrm{C}$, and $28{ }^{\circ} \mathrm{C}$ for 14 days. Oral exposures were replicated four times to reach 50 female mosquitoes for each population per temperature. In addition, directly after the blood meal a total of 20 engorged female mosquitoes of both populations were individually placed in $1.5 \mathrm{ml}$ SafeSeal micro tubes (Sarstedt, Germany) and stored at $-80^{\circ} \mathrm{C}$, to be able to determine the ingested WNV titers.

In order to bypass the midgut barrier and test mosquitoes with a known disseminated infection for the presence of a salivary gland barrier, mosquitoes were also infected with WNV through microinjections. Culex $p$. pipiens mosquitoes originating from The Netherlands and Italy were immobilized with $\mathrm{CO}_{2}$, and injected in the thorax with $69 \mathrm{nl}$ of WNV $\left(9.2 \pm 0.9 \times 10^{7} \mathrm{TCID}_{50} / \mathrm{ml}\right)$ using the Drummond Nanoject II AutoNanoliter Injector (Drummond Scientific Company, USA). Again, female mosquitoes from each group were divided over three separate buckets and placed at $18{ }^{\circ} \mathrm{C}$, $23^{\circ} \mathrm{C}$ and $28^{\circ} \mathrm{C}$ for 14 days. Infections were replicated 4 times to reach 50 female mosquitoes for each population per temperature. 


\section{Infection and transmission rates}

After the period of incubation, female mosquitoes were immobilized with $\mathrm{CO}_{2}$ and legs and wings were removed with forceps. The proboscis of each mosquito was inserted into a $200 \mu$ yellow pipette tip (Greiner bio-one, The Netherlands) containing $5 \mu \mathrm{l}$ of a 1:1 solution of FBS and $50 \%$ glucose solution, for a minimum period of 45 minutes. Each saliva sample was collected in a $1.5 \mathrm{ml}$ SafeSeal micro tube with $55 \mu \mathrm{l}$ of fully supplemented hepes-buffered DMEM medium, whereas bodies were stored in $1.5 \mathrm{ml}$ SafeSeal micro tubes with a small scoop of $0.5 \mathrm{~mm}$ zirconium beads (BioConnect BV, The Netherlands). All samples were stored at $-80^{\circ} \mathrm{C}$ until further use.

Frozen mosquito bodies were homogenized in the bullet blender storm (Next Advance, USA) for 2 minutes, and shortly spun down at 14,500 rpm in the Eppendorf minispin plus (Eppendorf, Germany). Next, $100 \mu \mathrm{l}$ of fully supplemented hepesbuffered DMEM medium was added and samples were again homogenized for 2 minutes in the Bullet blender, and spun down for 2 minutes at 14,500 rpm. For each mosquito homogenate or saliva sample, $30 \mu \mathrm{l}$ was incubated on a monolayer of Vero E6 cells in a 96 wells plate (Greiner Bio-one). After 2-3 hours the medium was completely removed and replaced by $100 \mu$ of hepes-buffered DMEM medium. After 3 days, each well was scored for WNV specific cytopathic effects. WNV titers were determined by endpoint dilution assays on Vero E6 cells, and scored for WNV specific cytopathic effects after 3 days.

\section{Statistical analysis}

Generalized linear models (GLM) with a binomial distribution and logit link function were used to test for the effects of population (The Netherlands versus Italy) and temperature on the WNV infection and transmission rates. Infection and transmission rates were calculated, respectively, by dividing the number of female mosquitoes with infected bodies or with infected saliva by the total number of female mosquitoes in the respective treatment. Effects of replicate, population, temperature, and the interaction term between population and temperature, were included in the model. If the interaction term was not significant, it was removed from the final model. Significant factors were further investigated with likelihood ratio tests (LRT). A Wilcoxon test was used to test for differences in the ingested WNV titers by both populations. Effects of population and temperature on viral titers were tested with Wilcoxon or Kruskal-Wallis (KWT) tests. Multiple comparisons were corrected with the Bonferroni procedure, by reducing the significance level from 0.05 to 0.017 . All statistical analyses were done with SAS software, version 9.3 (SAS Institute Inc., USA), and figures were made with the statistical software package $R$ ( $R$ Core Team 2016). 


\section{Results}

Effects of population and temperature on WNV vector competence of Dutch and Italian Cx. p. pipiens were investigated by orally exposing female mosquitoes to WNV, and incubating them for 14 days at $18^{\circ} \mathrm{C}, 23^{\circ} \mathrm{C}$, or $28^{\circ} \mathrm{C}$. The Dutch mosquito population ingested similar titers of $\mathrm{WNV}\left(3.6 \times 10^{5} \mathrm{TCID}_{50} / \mathrm{ml}\right)$ compared to the Italian mosquito population $\left(2.9 \times 10^{5} \mathrm{TCID}_{50} / \mathrm{ml}\right.$; Wilcoxon, $\mathrm{Z}=0.34$, df = $1, P=0.73$; Figure 1).

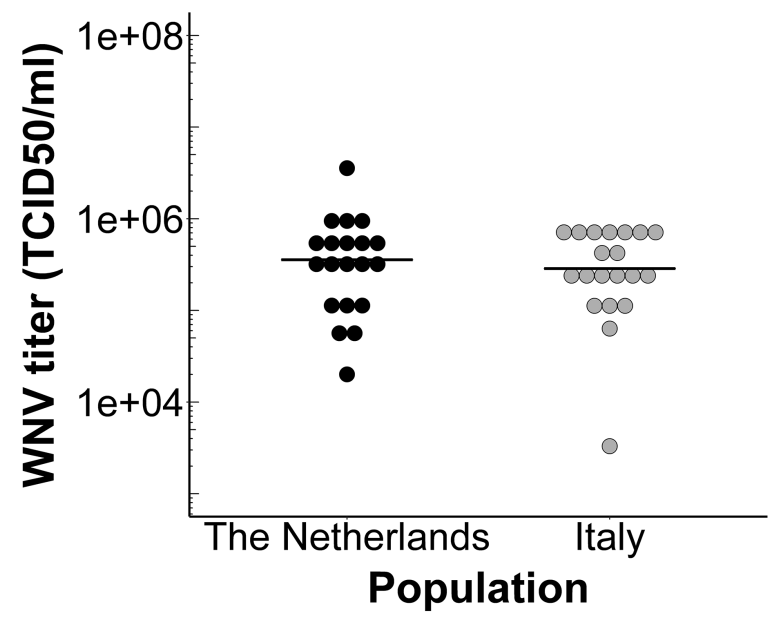

Figure 1: Ingested West Nile virus titers by Dutch and Italian Culex pipiens biotype pipiens mosquitoes. Viral titers were determined for engorged females originating from The Netherlands (black) and Italy (grey), which were directly frozen after feeding on the infectious blood. Each data point represents one engorged female mosquito. Horizontal black lines show median titers for each population.

After oral exposure, WNV infection rates of Dutch $C x$. p. pipiens females were 26 $\%$ at $18{ }^{\circ} \mathrm{C}, 34 \%$ at $23{ }^{\circ} \mathrm{C}$, and $32 \%$ at $28{ }^{\circ} \mathrm{C}$, and WNV infection rates of Italian females were $28 \%$ at $18{ }^{\circ} \mathrm{C}, 36 \%$ at $23{ }^{\circ} \mathrm{C}$, and $32 \%$ at $28{ }^{\circ} \mathrm{C}$ (Figure 2A). No significant effects were found on infection rates of population (GLM, $X^{2}=1.07, \mathrm{df}=1$, $P=0.30$ ), temperature (GLM $\left., X^{2}=1.67, \mathrm{df}=2, P=0.43\right)$, or the interaction between both (GLM, $X^{2}=0.03$, df $=2, P=0.99$ ). Thus, mosquito infection rates were similar for northern and southern European populations at all temperatures.

After oral exposure, WNV transmission rates of Dutch $C x$. p. pipiens females were 0 $\%$ at $18{ }^{\circ} \mathrm{C}, 10 \%$ at $23{ }^{\circ} \mathrm{C}$, and $10 \%$ at $28{ }^{\circ} \mathrm{C}$, and WNV transmission rates of Italian females were $0 \%$ at $18{ }^{\circ} \mathrm{C}, 2 \%$ at $23^{\circ} \mathrm{C}$, and $16 \%$ at $28{ }^{\circ} \mathrm{C}$ (Figure 2B). Temperature (GLM, $X^{2}=19.54, \mathrm{df}=2, P<0.001$ ) had a significant effect on transmission rates after oral exposure, whereas there was no significant effect of population (GLM, $X^{2}$ $=0.01, \mathrm{df}=1, P=0.92)$, or the interaction between both $\left(\mathrm{GLM}, \mathrm{X}^{2}=3.98 \mathrm{df}=2, P\right.$ $=0.14)$. As there was no population effect, differences between temperatures could 
be tested by pooling data from both populations. Pairwise comparisons between temperatures revealed that transmission rates were significantly lower at $18{ }^{\circ} \mathrm{C}$ compared to $28{ }^{\circ} \mathrm{C}$ (LRT, $\left.P<0.001\right)$, and at $18{ }^{\circ} \mathrm{C}$ compared to $23^{\circ} \mathrm{C}$ (LRT, $P=$ $0.003)$, but there was no significant difference between $23^{\circ} \mathrm{C}$ and $28^{\circ} \mathrm{C}(\mathrm{LRT}, P=$ 0.07). Thus, vector competence of northern and southern $C x$. p. pipiens populations was similar, and no WNV transmission was observed after 14 days incubation at 18 ${ }^{\circ} \mathrm{C}$.

A

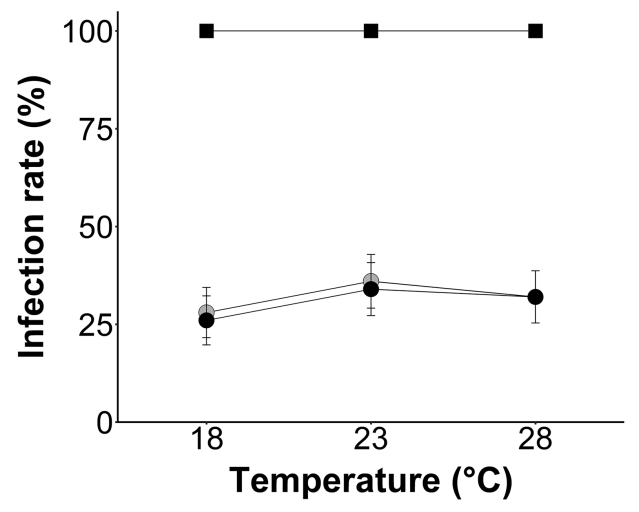

$\square$ Injection $\bigcirc$ Oral infection
B

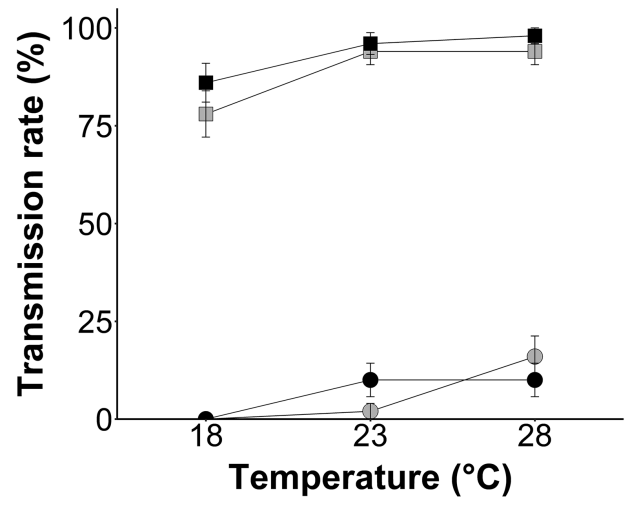

- The Netherlands 0 Italy

Figure 2: Effect of temperature on vector competence of Dutch and Italian Culex pipiens biotype pipiens mosquitoes for West Nile virus. (A) Infection rates and (B) transmission rates of Dutch (black) and Italian (grey) mosquitoes exposed orally (circles) or through intra-thoracic injections (squares). Mosquitoes were incubated for 14 days at $18^{\circ} \mathrm{C}, 23^{\circ} \mathrm{C}$, or $28^{\circ} \mathrm{C}$. Data points of infection rates after injections are overlapping for both populations. Each data point shows average infection rates for 50 female mosquitoes infected either orally or by injection with West Nile virus. Error bars show standard error of the means.

Mosquitoes were also infected with WNV through intra-thoracic injections in order to bypass the midgut, and investigate the effect of the salivary glands on virus dissemination. Infection and transmission rates were determined for the Dutch and Italian population at $18^{\circ} \mathrm{C}, 23^{\circ} \mathrm{C}$, or $28^{\circ} \mathrm{C}$ after 14 days of incubation. WNV infection rates were $100 \%$ for both populations, at all three temperatures (Figure $2 \mathrm{~A}$ ). WNV transmission rates of Dutch Cx. p. pipiens females were $86 \%$ at $18{ }^{\circ} \mathrm{C}, 96 \%$ at 23 ${ }^{\circ} \mathrm{C}$, and $98 \%$ at $28{ }^{\circ} \mathrm{C}$, and WNV transmission rates of Italian females were $78 \%$ at $18{ }^{\circ} \mathrm{C}, 94 \%$ at $23{ }^{\circ} \mathrm{C}$, and $94 \%$ at $28^{\circ} \mathrm{C}$ (Figure 2B). After injection, transmission rates were significantly different between temperatures $\left(\mathrm{GLM}, \mathrm{X}^{2}=14.21, \mathrm{df}=2, P\right.$ $<0.001)$, but not between populations $\left(\mathrm{GLM}, X^{2}=1.00, \mathrm{df}=1, P=0.32\right)$, nor for the interaction between both (GLM, $X^{2}=0.31$, $\mathrm{df}=2, P=0.86$ ). A similar pattern was observed as was found after oral exposure, with significantly lower transmission rates at $18{ }^{\circ} \mathrm{C}$ compared to $28{ }^{\circ} \mathrm{C}(\mathrm{LRT}, P<0.001)$, and at $18{ }^{\circ} \mathrm{C}$ compared to 23 
${ }^{\circ} \mathrm{C}(\mathrm{LRT}, P=0.003)$, but no significant difference between $23^{\circ} \mathrm{C}$ and $28^{\circ} \mathrm{C}$ (LRT, $P$ $=0.73$ ). Thus, after injection WNV transmission was lower at $18^{\circ} \mathrm{C}$ compared to 23 ${ }^{\circ} \mathrm{C}$ and $28^{\circ} \mathrm{C}$.

To explain the effect of temperature on vector competence, we determined viral titers of bodies of mosquitoes which had WNV in their saliva after 14 days of incubation, for orally exposed (Figure 3A) and injected female mosquitoes (Figure 3B). After 14 days of incubation, none of the orally exposed $C x$. p. pipiens females incubated at 18 ${ }^{\circ} \mathrm{C}$ were able to transmit WNV. Thus, WNV titers were only determined for females with positive body and saliva which were incubated at $23^{\circ} \mathrm{C}$ and $28^{\circ} \mathrm{C}$. Median WNV titers of orally exposed Dutch Cx. p. pipiens females were $2.0 \times 10^{5} \mathrm{TCI}_{50} / \mathrm{ml}$ at 23 ${ }^{\circ} \mathrm{C}$, and $6.3 \times 10^{5} \mathrm{TCID}_{50} / \mathrm{ml}$ at $28^{\circ} \mathrm{C}$, and median WNV titers of Italian females were $6.3 \times 10^{6} \mathrm{TCID}_{50} / \mathrm{ml}$ at $23{ }^{\circ} \mathrm{C}$, and $2.8 \times 10^{3} \mathrm{TCID}_{50} / \mathrm{ml}$ at $28{ }^{\circ} \mathrm{C}$ (Figure $3 \mathrm{~A}$ ). There was no significant difference in viral titers between both populations (Wilcoxon, $Z$ $=-1.83$, $\mathrm{df}=1, P=0.07$ ), nor between temperatures (Wilcoxon, $Z=1.43$, $\mathrm{df}=1$, $P=0.15)$ after oral exposure. However, it should be noted that as a result of low transmission rates, viral titers could only be determined from a limited number of positive mosquitoes. In general, WNV replicated to similar levels in the Dutch and Italian Cx. p. pipiens populations.

Viral titers of female mosquitoes that were infected with WNV through injections were also compared. Median WNV titers of injected Dutch $C x$. p. pipiens females were $5.4 \times 10^{5} \mathrm{TCID}_{50} / \mathrm{ml}$ at $18^{\circ} \mathrm{C}, 6.5 \times 10^{6} \mathrm{TCID}_{50} / \mathrm{ml}$ at $23^{\circ} \mathrm{C}$, and $2.8 \times 10^{6} \mathrm{TCID}_{50} /$ $\mathrm{ml}$ at $28^{\circ} \mathrm{C}$ (Figure 3B). Median WNV titers of injected Italian females were $3.9 \times 10^{5}$ $\mathrm{TCID}_{50} / \mathrm{ml}$ at $18{ }^{\circ} \mathrm{C}, 5.7 \times 10^{6} \mathrm{TCID}_{50} / \mathrm{ml}$ at $23{ }^{\circ} \mathrm{C}$, and $2.0 \times 10^{6} \mathrm{TCID}_{50} / \mathrm{ml}$ at $28{ }^{\circ} \mathrm{C}$ (Figure 3B). Again, there was no significant difference in viral titers between the two populations (Wilcoxon, $Z=0.64$, df $=1, P=0.52$ ), but there was a significant effect of temperature $\left(\mathrm{KWT}, \mathrm{X}^{2}=16.41 \mathrm{df}=2, P<0.001\right)$. Viral titers were significantly lower at $18{ }^{\circ} \mathrm{C}$ compared to $28^{\circ} \mathrm{C}$ (Wilcoxon, $Z=-3.58, \mathrm{df}=1, P<0.001$ ), and at 18 ${ }^{\circ} \mathrm{C}$ compared to $23^{\circ} \mathrm{C}$ (Wilcoxon, $\mathrm{Z}=-3.36, \mathrm{df}=1, P<0.001$ ), but not between 23 ${ }^{\circ} \mathrm{C}$ and $28^{\circ} \mathrm{C}$ (Wilcoxon, $Z=0.60, \mathrm{df}=1, P=0.55$ ). Thus, lower transmission rates at $18{ }^{\circ} \mathrm{C}$ after 14 days can be explained by lower viral titers inside mosquito bodies. 

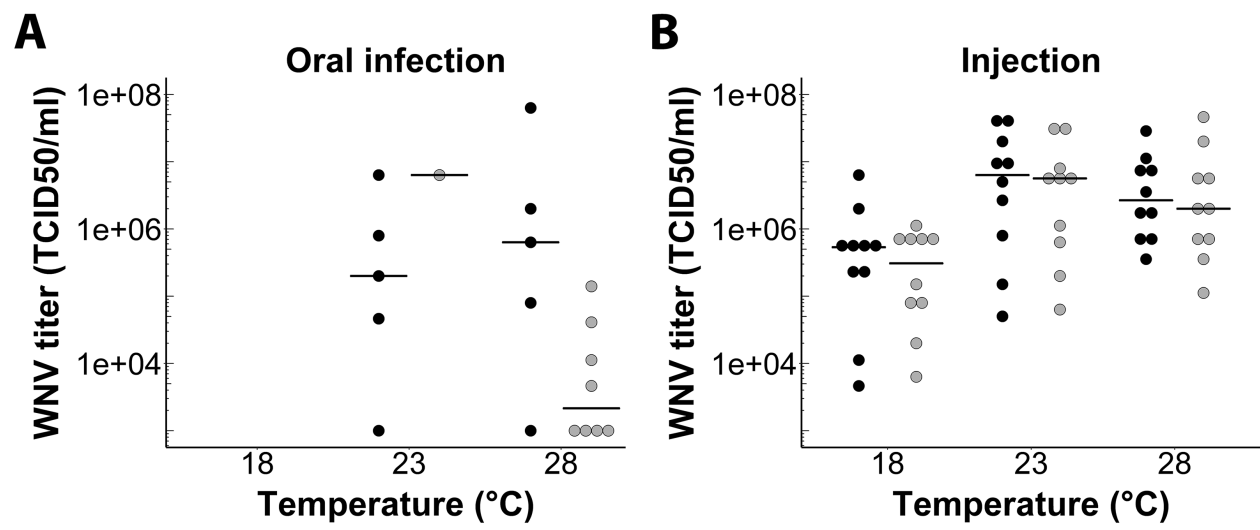

- The Netherlands ○ Italy

Figure 3: Effect of temperature on West Nile virus titers of Dutch and Italian Culex pipiens biotype pipiens mosquitoes with positive body and saliva. Viral titers of $(A)$ orally exposed and (B) injected Culex pipiens biotype pipiens mosquitoes originating from The Netherlands (black) or Italy (grey), determined after 14 days incubation at $18{ }^{\circ} \mathrm{C}, 23^{\circ} \mathrm{C}$, or $28^{\circ} \mathrm{C}$. Each data point represents one female mosquito with West Nile virus positive body and saliva. Horizontal black lines show median titers for each population per temperature.

\section{Discussion}

The aim of this study was to directly compare the vector competence of a northern and southern European $C x$. p. pipiens population for WNV across a gradient of temperatures. Overall, we found no difference in vector competence (infection and transmission rates) for WNV between the Dutch and Italian Cx. p. pipiens populations. However, we did find a positive effect of higher temperature on vector competence for both populations. Transmission of WNV after an infectious blood meal was only observed at $23^{\circ} \mathrm{C}$ and $28^{\circ} \mathrm{C}$, whereas no transmission was observed after 14 days of incubation at $18^{\circ} \mathrm{C}$.

Recently, several European studies determined vector competence of $C x$. pipiens populations originating from The Netherlands (Fros et al. 2015a, Fros et al. 2015b, Vogels et al. 2016a), Germany (Leggewie et al. 2016), Spain (Brustolin et al. 2016), and Italy (Fortuna et al. 2015a). However, due to the large diversity in experimental designs it is hard to make a direct comparison between outcomes of these studies. For instance, only two studies used molecularly identified biotype pipiens mosquitoes (Leggewie et al. 2016, Vogels et al. 2016a), whereas all other studies used the other biotype or did not differentiate between biotypes. Infection rates of the Dutch population at $18{ }^{\circ} \mathrm{C}$ were similar compared to previous studies on Dutch and German biotype pipiens populations, whereas infection rates at $23^{\circ} \mathrm{C}$ and $28^{\circ} \mathrm{C}$ were slightly lower in the present study (Leggewie et al. 2016, Vogels et al. 2016a). Transmission 
rates could not be compared with the German mosquito population, because no transmission rates were reported for this study (Leggewie et al. 2016). Transmission rates were similar in the present and our previous study on Dutch biotype pipiens mosquitoes, with no transmission at $18{ }^{\circ} \mathrm{C}$ after 14 days, and low transmission rates at $23^{\circ} \mathrm{C}$. Interestingly, previously recorded transmission rates were about three-fold higher at $28^{\circ} \mathrm{C}$ compared to this study, but the ratio between mosquitoes with infected saliva and mosquitoes with infected bodies is comparable. This indicates an overall lower success of initial infection in the present study, which may be due to the midgut infection barrier. Such differences are likely due to variation between populations or temporal variation in vector competence (Kilpatrick et al. 2010, Vaidyanathan \& Scott 2007). Both infection and transmission rates of the Italian population in this study were lower compared to a previous study with a $C x$. pipiens population in Italy (Fortuna et al. 2015a). However, the same study showed significant differences in infection rates among different $C x$. pipiens populations in Italy, which again suggests that vector competence is a variable trait.

Increased vector competence at higher temperatures has been described for several arthropod-borne viruses and their respective mosquito vectors (Fros et al. 2015b, Kilpatrick et al. 2008, Reisen et al. 1993, Zouache et al. 2014). Higher temperatures increase viral replication, which can result in shorter extrinsic incubation periods and higher infection and transmission rates (Chamberlain \& Sudia 1961, Hardy et al. 1983). However, temperature may also affect the midgut barrier or antiviral responses such as RNAi (Adelman et al. 2013, Alto \& Bettinardi 2013). Thus, complex interactions between virus and mosquito may shape the relation between temperature and vector competence.

In the present study, we did not find any transmission after 14 days of incubation at $18{ }^{\circ} \mathrm{C}$. However, it is possible that mosquitoes required longer extrinsic incubation periods before WNV could be transmitted at $18^{\circ} \mathrm{C}$. Previous studies showed that at relatively low temperatures the extrinsic incubation period increases to 15-22 days, depending on the specific combination between mosquito species and WNV strain (Kilpatrick et al. 2008, Reisen et al. 2006). Thus, European Cx. p. pipiens might be competent vectors for WNV when incubated for longer periods at $18^{\circ} \mathrm{C}$. Future studies should determine the minimum extrinsic incubation periods of European $C x$. pipiens populations for WNV at different temperatures.

In this study, mosquitoes were reared and kept at constant temperatures. However, daily fluctuations in temperature may have consequences for virus transmission. Previous studies on dengue virus showed that daily fluctuations in temperature can both result in increased as well as decreased virus transmission, depending on the temperature range and mean temperature that virus-exposed mosquitoes 
experience (Lambrechts et al. 2011). In contrast, two studies on the effect of temperature fluctuations on WNV transmission showed no difference between mean and fluctuating temperatures (Cornel et al. 1993, Danforth et al. 2016). Thus, results from experiments on WNV with constant temperature seem to provide reliable estimates for vector competence, but should be interpreted with care nevertheless.

Here, we showed that temperature, but not population origin, had an effect on vector competence of $C x$. p. pipiens mosquitoes for WNV. Despite variation in vector competence of different European populations (Fortuna et al. 2015a, Leggewie et al. 2016, Vogels et al. 2016a), our findings show that temperature is the main factor that can explain differences in WNV outbreaks between northern and southern Europe. Interestingly, we did not observe any transmission of WNV by both populations after 14 days of incubation at a temperature of $18{ }^{\circ} \mathrm{C}$, which is representative for an average northern European summer (Haylock et al. 2008). Although this does not exclude possible transmission after longer incubation periods, it shows that transmission is inefficient at low temperature. This can explain the absence of WNV outbreaks in northern European countries (Chancey et al. 2015). Although the current risk for WNV transmission in northern Europe seems low, circulation is possible under favourable climatic conditions due to the presence of competent mosquito vectors (Vogels et al. 2016a, Vogels et al. 2015), and susceptible bird hosts (Lim et al. 2015, Lim et al. 2014). Models are needed to assess future risks of WNV transmission in northern Europe, especially in the light of more frequent and longer periods of intense heat due to global warming (chapter 7 of this thesis).

\section{Conclusions}

Differences in WNV outbreaks between northern and southern Europe cannot be explained by intrinsic differences in vector competence between $C x$. p. pipiens populations from both regions. In addition, no transmission was observed after 14 days of incubation at the average summer temperature of $18^{\circ} \mathrm{C}$ of northern Europe, whereas both the Dutch and Italian $\mathrm{Cx}$. p. pipiens population were able to transmit WNV at similar rates at $23^{\circ} \mathrm{C}$ and $28^{\circ} \mathrm{C}$. This suggests that temperature is a key limiting factor for WNV transmission in northern Europe.

\section{Acknowledgements}

We thank Tim Möhlmann, Claudia Damiani, and Bep Vogels for support in collection of Culex egg rafts, Corinne Geertsema for maintenance of cell cultures, Gerrit Gort for support with statistical analyses, and Marcel Dicke for providing comments on an earlier version of this chapter. 



\section{Chapter 6}

\section{Virus interferes with host-seeking behaviour of mosquito}

Chantal B.F. Vogels ${ }^{1}$, Jelke J. Fros ${ }^{2,3}$, Gorben P. Pijlman², Joop J.A. van Loon', Gerrit Gort ${ }^{4}$, and Constantianus J.M. Koenraadt ${ }^{1}$

${ }^{1}$ Laboratory of Entomology, Wageningen University \& Research

${ }^{2}$ Laboratory of Virology, Wageningen University \& Research

${ }^{3}$ Nuffield Department of Medicine, University of Oxford

${ }^{4}$ Biometris, Wageningen University \& Research

Journal of Experimental Biology (in press) 


\begin{abstract}
Transmission of vector-borne pathogens is dependent on host-seeking behaviour of their vector. Pathogen manipulation of host-seeking behaviour of vectors towards susceptible hosts is supposedly beneficial for transmission. For West Nile virus (WNV), manipulation of the host-seeking behaviour of the main mosquito vector towards birds would be advantageous, because mammals are dead-end hosts. We hypothesized that WNV infection induces a stronger host-seeking response and a shift in host preference towards birds, to enhance its transmission by mosquitoes. However, here we show that WNV infection decreases the host-seeking response, and does not induce a shift in mosquito host preference. Other fitness-related traits are not affected by WNV infection. No effect of WNV infection was found on antennal electrophysiological responsiveness. The reduced host-seeking response is, thus, likely resulting from interference in the mosquito's central nervous system. This is the first study that shows changes, specifically in the host-seeking behaviour induced by a pathogen, that are not favouring transmission.
\end{abstract}

Keywords: manipulation, host preference, host-seeking, virus transmission, olfaction 


\section{Introduction}

The successful transmission of pathogens that are spread by arthropods from one host to another, depends on complex interactions between pathogen, arthropod vector, host, and the environment (Gray \& Banerjee 1999, Weaver \& Barrett 2004). The natural transmission cycle can only be completed if an infectious vector is able to find a new susceptible host and transmit the pathogen while feeding. Many examples exist of pathogens that influence host feeding behaviour of its vector, in a way that seems to increase their transmission (van Houte et al. 2013). Increased transmission can be achieved through direct manipulation of vector behaviour or through indirect effects of the pathogen on, for instance, host defence or host attractiveness (De Moraes et al. 2014, Hurd 2003, Targett 2006, van Houte et al. 2013).

Manipulation can occur during two stages of the vector's host-feeding behaviour: the host-seeking and the feeding stage. Manipulation during these two stages has been shown for various medically important pathogens that are transmitted by mosquitoes (Hurd 2003, Lefèvre \& Thomas 2008). During the first stage, mosquitohost contact can be increased by induction of a stronger mosquito host-seeking response to host odour (Cator et al. 2013, Koella et al. 2002, Rossignol et al. 1986, Smallegange et al. 2013), or a stronger host preference for suitable hosts (Lefèvre et al. 2006). Pathogens can induce such changes by altering the mosquito's odour perception, because host odours, together with carbon dioxide, are important cues for mosquitoes to locate a host (Dekker et al. 2005, Takken 1991). The second stage starts after the vector has encountered a host. Increased probing and lowered feeding performance (Grimstad et al. 1980, Lima-Camara et al. 2011), which leads to longer and more frequent feedings, are a second way by which the pathogen's transmission rates can be increased. However, despite this information, evidence that changes in mosquito behaviour are actually due to direct manipulation by the pathogen remains scarce. Moreover, a recent study showed that stimulation of mosquitoes with heat-killed Escherichia coli induced similar behavioural changes as were observed after infection with the Plasmodium parasite (Cator et al. 2013). This suggests that indirect manipulation due to immune challenge, rather than direct manipulation by the pathogen, underlies the behavioural change (Cator et al. 2013). More studies on mechanisms of manipulation are, therefore, needed to fully understand the direct and indirect effects of pathogen infection on vector behaviour.

Up to now, several studies investigated the effect of infection with animal- or plantviruses on vector feeding behaviour (Blanc \& Michalakis 2016, Hurd 2003, van Houte et al. 2013). Studies have mainly focused on host-choice (e.g. attraction of aphids to plants infected with Barley yellow dwarf virus (Ingwell et al. 2012)) or feeding behaviour (e.g. increased probing behaviour of mosquitoes infected with La Crosse 
virus (Grimstad et al. 1980)). However, no studies have investigated the effect of viruses on changes in vector behaviour during the host-seeking stage. Here, we investigated whether West Nile virus (WNV; family: Flaviviridae) infection can induce changes in the host-seeking response and host preference of the mosquito Culex (Cx.) pipiens. Culex pipiens mosquitoes maintain WNV in an enzootic cycle with birds, whereas mammals are dead-end hosts (Hayes et al. 2005). From the perspective of WNV, manipulation of the host-seeking response and host preference towards birds is, therefore, beneficial for its transmission. Especially $C x$. pipiens biotype pipiens is an important vector for WNV due to its preference for birds (Fritz et al. 2015, Osório et al. 2012). We hypothesized that WNV-infected Cx. pipiens biotype pipiens mosquitoes have a stronger host-seeking response, and have a preference shifted towards avian hosts compared to uninfected mosquitoes. In order to test this hypothesis, we investigated the effect of WNV infection on host-seeking behaviour, by determining the host-seeking response and preference in an olfactometer. We also investigated mosquito flight activity, blood feeding, and survival, in order to control for indirect effects of WNV infection on the mosquito's fitness. To understand the underlying mechanisms of changes in the host-seeking behaviour we investigated antennal olfactory responsiveness of uninfected and WNV-infected mosquitoes.

\section{Materials and Methods}

\section{Mosquitoes}

Culex pipiens biotype pipiens was established in the laboratory in the summer of 2014 by collecting egg rafts from aboveground rainwater barrels in Best, The Netherlands. One larva from each egg raft was identified up to the biotype level with a real-time PCR assay (Vogels et al. 2015). Larvae from 162 egg rafts identified as biotype pipiens where grouped in trays $(25 \mathrm{~cm} \times 25 \mathrm{~cm} \times 8 \mathrm{~cm})$. Trays were filled with tap water and a drop of Liquifry No. 1 (Interpet Ltd., UK) was added. Thereafter, larvae were fed daily with a 1:1:1 mixture of bovine liver powder, ground rabbit food and ground koi food. Pupae were transferred to Bugdorm cages $(30 \mathrm{~cm} \times 30 \mathrm{~cm} \times 30$ $\mathrm{cm}$ ) and provided ad libitum with $6 \%$ glucose solution. Bovine or chicken blood was provided through a Hemotek PS5 (Discovery Workshops, UK) feeder to allow for egg production by the mosquitoes. Larvae and adults were maintained at $23^{\circ} \mathrm{C}$ with 16:8 L:D cycle and $60 \% \mathrm{RH}$. Lights turned on at $3 \mathrm{pm}$, and turned off at $7 \mathrm{am}$. The first generation (F1) mosquitoes reared in the laboratory were used for experiments. Female mosquitoes were kept together with males for 3 to 6 days before being transferred to the Biological Safety Level 3 (BSL3) facility for experiments. 


\section{Virus}

In all experiments a passage 2 stock of West Nile virus lineage 2 (GenBank accession no. HQ537483.1) originating from Greece (2010) was used with a $50 \%$ tissue culture infective dose of $1.12 \times 10^{9} \mathrm{TCID}_{50} / \mathrm{ml}$. WNV was grown as described before (Fros et al. 2015a, Vogels et al. 2016a).

\section{Mosquito infection with West Nile virus}

Three to six day old female mosquitoes were immobilised with $\mathrm{CO}_{2}$ and injected with $69 \mathrm{nl}(46 \mathrm{nl} / \mathrm{sec})$ of phosphate buffered saline (mock infection) or $1.12 \times 10^{9}$ $\mathrm{TCID}_{50} / \mathrm{ml}$ of WNV (WNV infection) using the Nanoject II Auto-Nanoliter Injector (Drummond scientific company, USA). Mosquitoes in the control treatment were only immobilised with $\mathrm{CO}_{2}$. Mosquitoes were provided ad libitum with $6 \%$ glucose solution and maintained at $23{ }^{\circ} \mathrm{C}, 16: 8 \mathrm{~L}: \mathrm{D}$ cycle and $60 \% \mathrm{RH}$. Mosquitoes were used in experiments 8 to 10 days post injection in order to make sure that all mosquitoes had WNV disseminated to the salivary glands, and thus were able to transmit WNV (Fros et al. 2015a, Vogels et al. 2016a).

\section{Test odour blends and individual compounds}

For the behavioural assays, odours were collected from three humans by wearing a nylon sock (20 Den panty sock, Hema, The Netherlands) for $24 \mathrm{~h}$ and from five chickens by tying a nylon sock around the leg for $24 \mathrm{~h}$. Chicken odour collections were approved by the Animal Ethics Committee of Wageningen University (DEC protocol 2013113.b), and all methods were performed in accordance with the relevant guidelines and regulations. Each sock was cut in three equal pieces. Three pieces of three different human or chicken individuals were combined and used as stimulus in the one-port olfactometer. Each set of socks was used during three experimental mornings. Socks were stored in glass jars at $-20^{\circ} \mathrm{C}$ until use.

For the electrophysiological assays, geranylacetone (96\%, Aldrich, Germany), hexanoic acid (99\%, Sigma, Germany), and nonanal (95\%, Aldrich, Germany) were selected as volatile chemicals. These volatiles were selected because they are present in odour profiles of human and chicken, they bind to different olfactory receptors, and they elicit high responses in antennae of $C x$. quinquefasciatus (Puri et al. 2006, Syed \& Leal 2009). All three volatiles were diluted to a $1 \%$ concentration in dichloromethane (>99.9\%, Sigma, Germany). Of each diluted volatile or the solvent, $20 \mu \mathrm{l}$ was applied on a piece of Whatman filter paper $(5 \mathrm{~mm} \times 40 \mathrm{~mm})$, left for evaporation of the solvent at room temperature, and then inserted in a Pasteur pipet which was sealed at both ends with Parafilm. New Pasteur pipettes with volatiles were prepared every experimental morning. 


\section{Behavioural assay}

Host-seeking responses of control, mock-infected, and WNV-infected female mosquitoes were tested in a one-port olfactometer $(1.65 \mathrm{~m} \times 0.65 \mathrm{~m} \times 0.65 \mathrm{~m})$ under BSL3 conditions (Braks \& Takken 1999, Knols et al. 1994). Four to five days prior to experiments, water was provided to female mosquitoes in order to induce the host-seeking response. The last day before being tested in the olfactometer, female mosquitoes were individually transferred to $50 \mathrm{ml}$ tubes with a mesh bottom, without access to water. The olfactometer was specifically designed for safe release of individual mosquitoes from the modified $50 \mathrm{ml}$ tubes. A piece of Velcro was mounted on the lid of the tubes, and a complementary piece of Velcro was mounted on the release mechanism inside the olfactometer. Tubes were inserted in an opening in the middle of one end of the olfactometer, and after closing the olfactometer, they could be safely opened via the release mechanism on the outside of the olfactometer. Individually released mosquitoes were allowed to respond to the odour stimulus by flying through the port at the other end, within a time period of 7 minutes. For all three odour stimuli (control, human, and chicken), $5 \% \mathrm{CO}_{2}(450 \mathrm{ml} / \mathrm{min}$ ) was released from a circular release point placed directly in front of the port. A trapping device containing a Hemotek PS5 feeder for heat production was placed behind the port. For the control stimulus no sock was added, for the human odour stimulus three one-third pieces of socks worn by humans were wrapped around the feeder, and for the chicken odour stimulus three one-third pieces of socks worn by chicken were wrapped around the feeder. Charcoal-filtered and moistened air was fed through the trapping device at a speed of $0.22 \pm 0.02 \mathrm{~m} / \mathrm{s}$. Temperature in the experimental room was set at $24{ }^{\circ} \mathrm{C}$ and relative humidity at $70 \%$. Experiments were performed during the first hours of the dark phase, with red light and dimmed light turned on. Each of the nine combinations of mosquito treatment and odour stimulus was replicated with 53-57 individual female mosquitoes.

For biosafety reasons (i.e. free-flying WNV-infected mosquitoes in an olfactometer), the order of testing the three mosquito treatments (control, mock-, WNV-infected) was kept the same. In addition, the order of odour stimuli was alternated during consecutive experimental mornings according to a Latin square design. Before each stimulus was tested, temperature and relative humidity were measured in the room, the middle of the olfactometer and the port from which the stimulus was released. There should be a gradient from room to port in both temperature and relative humidity in order to attract mosquitoes to the port. Mosquitoes that had entered the trapping device were stored in Eppendorf tubes at $-80^{\circ} \mathrm{C}$, and tested for WNV infection (Fros et al. 2015a). 


\section{Mosquito fitness-related parameters}

Flight duration was determined for a selection of control, mock-infected, and WNVinfected female mosquitoes tested in the behavioural assay. Flight duration was recorded for 41 control females, 43 mock-infected females, and 39 WNV-infected females, which were released in a one-port olfactometer. The fraction of time in flight was calculated as flight duration divided by total time, with total time being the time between the moment of release and the moment the mosquito entered the trapping device containing odour, or 7 minutes if the mosquito did not respond.

Blood feeding propensity was determined by offering chicken whole blood through the Hemotek P5 feeder to control, mock-infected, and WNV-infected female mosquitoes. Fifteen female mosquitoes of each treatment were placed together in a plastic bucket $(\varnothing=12.5 \mathrm{~cm}$ and height $=12 \mathrm{~cm})$ and were allowed to blood feed for 1 hour. Blood feeding propensity was tested at 7 to 9 days post injection. The experiment was replicated four times.

Survival was determined by placing ten control, ten mock-infected, and ten WNVinfected female mosquitoes in three separate buckets directly after the injection procedure. Mosquitoes were provided with $6 \%$ glucose solution and maintained at $23{ }^{\circ} \mathrm{C}$ with 16:8 L:D cycle and $60 \% \mathrm{RH}$. The number of surviving female mosquitoes was counted daily for 30 days. The experiment was replicated three times.

\section{Electrophysiology}

Electroantennography (EAG) was used to record responses of antennae of control, mock-infected, and WNV-infected female mosquitoes to the three volatile compounds and the solvent under BSL3 conditions. One day before EAG-recording, female mosquitoes were individually transferred to tubes without access to glucose solution. Female mosquitoes were individually immobilised with $\mathrm{CO}_{2}$, when being prepared for EAG recordings. EAG recordings were done following the method described by Qiu et al. (2013). For each antenna of an individual female only the solvent and one test volatile were recorded at one min intervals to prevent serial effects. In total, 10 female mosquitoes of each treatment (control, mock-infected, and WNV-infected) were tested for each of the three volatile odours ( $1 \%$ geranylacetone, $1 \%$ hexanoic acid, and $1 \%$ nonanal). Standardised responses were calculated by dividing the absolute response amplitude $(\mathrm{mV})$ to the volatile compound by the response amplitude $(\mathrm{mV})$ of the same antennae to the solvent and multiplying by 100 .

\section{Statistical analysis}

A generalized linear mixed model with a binomial distribution and logit link function was used to test for the effect of WNV infection and odour stimulus on the proportion 
of responding mosquitoes in the behavioural assay. WNV infection, odour stimulus and the interaction between WNV infection and odour stimulus were included in the model as fixed effects. To account for the experimental design, random effects were included for days and blocks of six consecutive measurements within day in which the stimulus was held fixed.

A linear mixed model was used to test for the effect of WNV infection and odour stimulus on flight activity. The same fixed and random effects as mentioned above for the behavioural assay were included in the model. Degrees of freedom were calculated according to the method of Kenward-Roger (Kenward \& Roger 1997).

A generalized linear mixed model with a binomial distribution and logit link function was used to test for differences in the proportion of blood-fed female mosquitoes over the three treatments (control, mock infection and WNV infection). Random effects were included for replicates. Observation-level random effects were included to handle overdispersion (Harrison 2014).

Kaplan Meier survival analysis was used to test for differences in survival of mosquitoes exposed to the three treatments.

Electroantennogram recordings were analysed with EAG pro software version 1.1 (Syntech, Germany).

Kruskal-Wallis tests were used to test for the effect of WNV infection on the standardized median responses for each volatile odour. All data were analysed in the statistical software package $R$ ( $R$ Core Team 2016).

\section{Results}

\section{West Nile virus decreases mosquito's host-seeking response}

To test whether WNV infection leads to a stronger host-seeking response and a shift in preference towards birds, female mosquitoes were individually exposed to host odours in a one-port olfactometer. There was a significant effect of WNV infection $(P=0.003)$ and odour stimulus $(P<0.001)$ on the host seeking response, but no interaction between both $(P=0.61)$. The interaction term was, therefore, excluded from the final model. Control, mock-infected, and WNV-infected mosquitoes had a similar preference for chicken odour. Thus, WNV infection did not induce a shift in host preference.

Female mosquitoes infected intra-thoracically with WNV showed an overall lower host-seeking response $(4.3 \%)$, independent of odour stimulus, compared to mockinfected $(14.4 \% ; P=0.010)$, and control females (13.8\%; $P=0.012$; Figure $1 \mathrm{~A})$. The infection procedure did not affect the response, because there was no difference in 
response between control females and mock-infected females ( $P=1.00$; Figure 1A). All female mosquitoes injected with WNV were confirmed to be infected with WNV, by testing them with a WNV infectivity assay (Fros et al. 2015b, Vogels et al. 2016a). Thus, independent of the odour stimulus offered, WNV infection decreased the hostseeking response of its mosquito vector.

Chicken odour in combination with $\mathrm{CO}_{2}$ and heat attracted a significantly higher proportion of female mosquitoes (20.0\%), independent of WNV infection, than human odour in combination with $\mathrm{CO}_{2}$ and heat $(9.1 \% ; P=0.026)$, and $\mathrm{CO}_{2}$ and heat only $(3.6 \% ; P<0.001$; Figure $1 \mathrm{~B})$. The addition of human odour did not attract a higher proportion of female mosquitoes than $\mathrm{CO}_{2}$ and heat alone $(P=0.099$; Figure 1B). Thus, both uninfected and infected female mosquitoes had a preference for chicken odour.

A

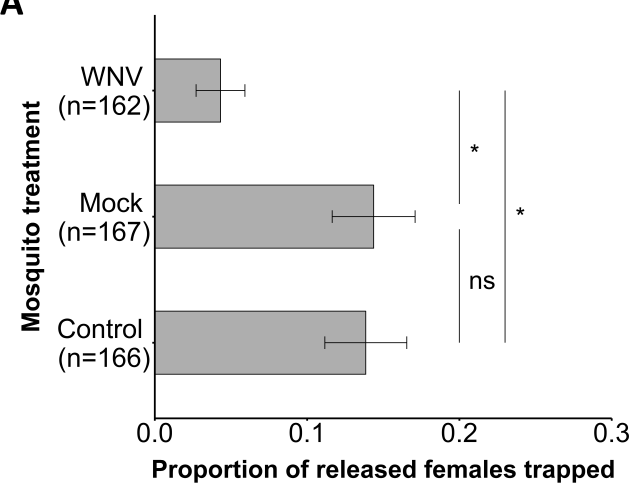

B

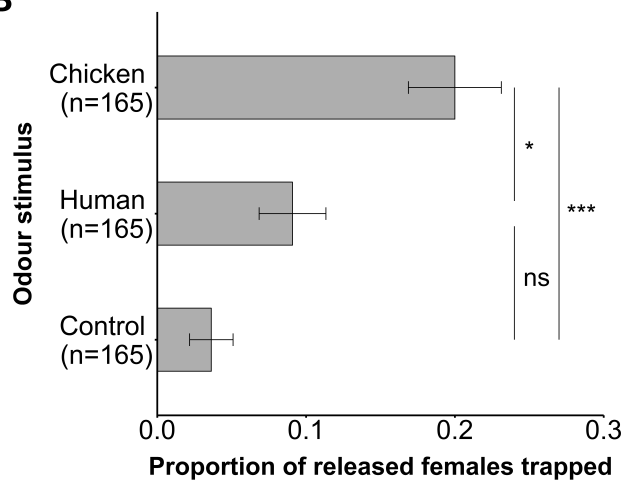

Figure 1: Host-seeking response of control, mock-infected, and West Nile virus-infected Culex pipiens biotype pipiens females to odour stimuli in the one-port olfactometer. (A) Proportion of released female mosquitoes trapped per infection treatment (control, mock-infected, and West Nile virus-infected) pooled for all three odour stimuli (control, human odour, chicken odour). (B) Proportion of released female mosquitoes trapped per odour stimulus (chicken, human, and control) pooled for infection treatment. Sample size is indicated for each treatment and odour stimulus. ns = not significant, ${ }^{*}=p<0.05,{ }^{* * *}=p<0.001$. Error bars show the standard error of the mean.

\section{No effect of West Nile virus infection on other mosquito fitness-related parameters}

Flight activity, blood feeding propensity, and survival were determined in order to control for possible negative effects of WNV infection on mosquito fitness. Flight duration was recorded when mosquitoes were released in the olfactometer (Braks \& Takken 1999, Knols et al. 1994). Median fractions of time in flight were 0.76 for control, 0.75 for mock-infected, and 0.75 for WNV-infected female mosquitoes, when pooled over the three odour stimuli (Figure 2A). No significant effects were found in 
these fractions of time in flight, neither of treatment $\left(\mathrm{F}_{2,109}=0.30, P=0.74\right)$, nor of odour stimuli $\left(\mathrm{F}_{2,108}=0.30, P=0.74\right)$, nor of their interaction $\left(\mathrm{F}_{4,109}=0.50, P=0.74\right)$. Thus, WNV infection does not influence flight activity.

We investigated the effect of WNV infection on the mosquito's propensity to take a blood meal. In total $25.4 \%$ of control females, $31.6 \%$ of mock-infected females, and $31.7 \%$ WNV-infected females took a blood meal (Figure 2B). These proportions were not significantly different $\left(X^{2}=0.65\right.$, df $\left.=2, P=0.72\right)$. The variance among replicates, however, was considerable $\left(X^{2}=6.040,50: 50\right.$ mixture of $X_{0}^{2}$ and $X_{1}^{2}, P$ $=0.007$ ). Thus, WNV infection does not significantly influence the propensity of mosquitoes to take a blood meal, though this propensity varies over time.

The effect of WNV infection on mosquito survival was monitored during 30 days. Thirty days post infection, $100 \%$ of the control females, $93 \%$ of the mock-infected females, and $83 \%$ of the WNV-infected females were still alive (Figure 2C). These differences tended to significance $\left(X^{2}=5.70\right.$, $\left.d f=2, P=0.057\right)$. Thus, WNV infection had a minor effect on mosquito survival.
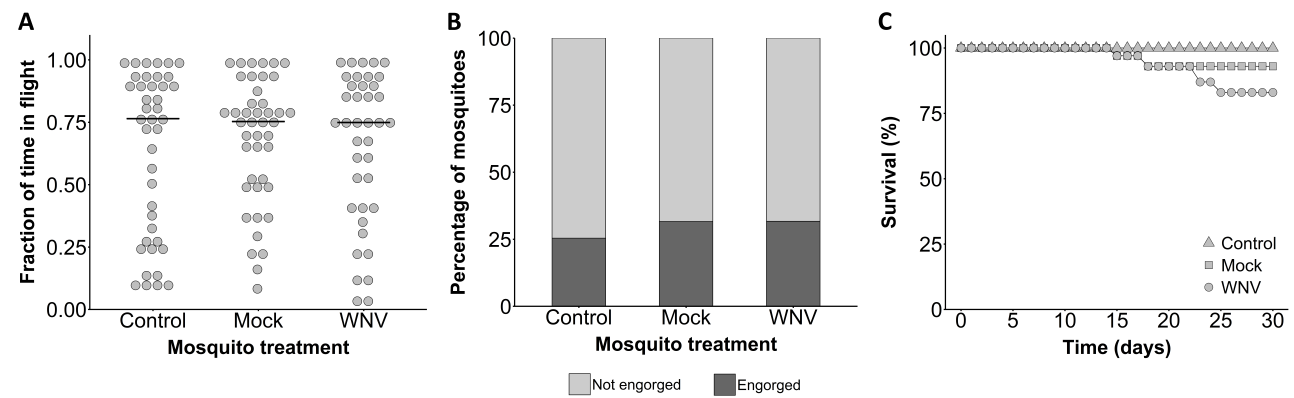

Figure 2: Mosquito fitness parameters flight activity, blood feeding propensity and survival of control, mock-infected, and West Nile virus-infected Culex pipiens biotype pipiens females. $(A)$ Fraction of time in flight in the behavioural assay, pooled for the three odour stimuli. Each dot represents one mosquito. Horizontal black lines indicate the median fraction of time in flight. (B) Percentage of bloodfed female mosquitoes based on four replicates of 15 females per infection treatment. (C) Percentage survival over a time period of 30 days post infection. Each treatment consisted of three replicates of 10 female mosquitoes.

\section{West Nile virus does not affect antennal olfactory responsiveness}

Electroantennogram responses of uninfected and infected female mosquitoes to three host-derived volatile compounds were compared to determine the effect of WNV infection on the sensitivity of antennal olfactory neurons (Qiu et al. 2013). There were no significant differences between the standardized antennal responses of control, mock-infected, and WNV-infected female mosquitoes to $1 \%$ geranyl 
acetone $\left(X^{2}=0.28, d f=2, P=0.87 ;\right.$ Figure $\left.3 A\right), 1 \%$ hexanoic acid $\left(X^{2}=0.03, d f=\right.$ $2, P=0.99$; Figure $3 \mathrm{~B})$, and $1 \%$ nonanal $\left(X^{2}=0.52, \mathrm{df}=2, P=0.77\right.$; Figure $\left.3 \mathrm{C}\right)$. Thus, there is no indication that WNV infection interferes with the peripheral olfactory system.
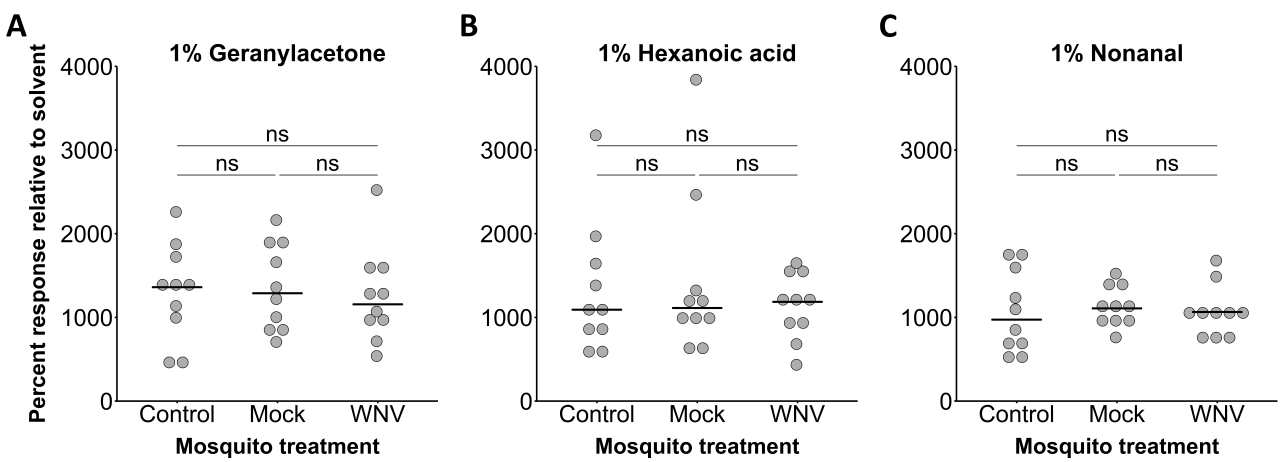

Figure 3: Standardised antennal responses of control, mock-infected, and West Nile virus-infected Culex pipiens biotype pipiens females for three different host-derived volatiles. Percent responses of control, mock-infected, and West Nile virus-infected female mosquitoes to (A) $1 \%$ geranyl acetone, (B) $1 \%$ hexanoic acid, and (C) $1 \%$ nonanal, relative to the solvent dichloromethane. Each odour was tested on antennae of ten female mosquitoes per infection treatment. Antennal responses were standardised by dividing the absolute response $(\mathrm{mV})$ of an antenna to the volatile compound by the response $(\mathrm{mV})$ to the solvent. Each dot represents one tested antenna. Horizontal black lines indicate the median percent response. $\mathrm{ns}=$ not significant.

\section{Discussion}

WNV infection decreased the host-seeking response of its mosquito vector, and did not induce a shift in host preference. As other fitness-related parameters (flight activity, blood feeding propensity, and survival) were not affected, we investigated the effect of WNV infection on the mosquito's olfactory response. No effect was found of WNV infection on electrophysiological responses in the peripheral olfactory system. The reduced host-seeking response is, thus, likely due to interference of WNV infection with the function of the mosquito's central nervous system.

Infection of nervous tissues occurs 8 days after $C x$. quinquefasciatus becomes infected with WNV through an infectious blood meal (Girard et al. 2004). WNV has been isolated from all nervous tissues of the mosquito including the brain, thoracic ganglia, abdominal ganglia, cephalic ganglion, and Johnston's organ (Girard et al. 2004, Girard et al. 2005). In our study, behavioural assays were done with female mosquitoes at 8 to 10 days post WNV injections. At this moment, WNV must have infected the mosquito brain already. We hypothesize, therefore, that WNV interferes with the processing of signals from the olfactory neurons in the brain. Further 
investigation of the mosquito brain is needed to unravel mechanisms underlying the effects of WNV infection on the mosquito's host-seeking behaviour.

An alternative explanation for the observed decrease in host-seeking response may be found in the mosquito's immune system. Studies with the malaria vector An. stephensi showed that an increase of the host-seeking response could also be induced by stimulation with heat-killed bacteria (Cator et al. 2013). Thus, manipulation may be an indirect result of immune challenge, rather than direct manipulation due to infection with the malaria parasite. It would therefore be interesting to further investigate the effect of immune challenge on other mosquito species such as $C x$. pipiens. Of particular interest would be the effects of inoculation with non-infectious WNV on the mosquito's host-seeking response.

The reduced host-seeking response implies that WNV-infected $C x$. pipiens mosquitoes would be less likely to localize a host in nature, which could consequently reduce the chances of successful enzootic WNV transmission. To our knowledge this is the first study that shows changes in host-seeking behaviour of a vector induced by a pathogen, that are not favouring transmission, but which seems to be detrimental for both pathogen transmission and fitness of the vector. However, given the repeated outbreaks of WNV, WNV transmission in nature is not reduced to the extent that it halts transmission. In order to explain the ongoing transmission of WNV, we hypothesize that there is a trade-off between WNV dissemination and infection of secondary organs, including the brain. We expect that this trade-off is dose-dependent and driven by the level of WNV replication in the mosquito. WNV replication in the mosquito depends on the ingested WNV dose, the mosquito's antiviral response, and the effectiveness of tissue barriers such as the midgut and salivary glands which can be dose-dependent (Franz et al. 2015). Relatively high levels of WNV replication inside the mosquito, e.g. due to a high dose of ingested WNV (Anderson et al. 2010, Sardelis et al. 2001), increase the chances of dissemination to the saliva, which favours transmission. However, dissemination also leads to infection of secondary organs such as the brain (Girard et al. 2004, Girard et al. 2005), which decreases the mosquito's host-seeking response, resulting in lowered WNV transmission. At relatively low levels of WNV replication, chances of dissemination to the saliva are lower due to dose-dependent barriers (Franz et al. 2015), thus, there is a lower chance of WNV infection in the brain. The mosquito's host-seeking response is, therefore, likely not decreased. In nature, this trade-off between dissemination and infection of the brain is expected to reach an equilibrium due to stabilizing natural selection on intermediate WNV replication levels, which may explain the ongoing repeated outbreaks of WNV. Further research is needed to investigate the effect of different WNV doses on the host-seeking response of $C x$. pipiens mosquitoes. 
In contrast to other mosquito species which readily respond to host odours in an olfactometer set-up (Allan et al. 2010, Pates et al. 2001), Cx. pipiens mosquitoes were less motivated to respond. Therefore, we deprived mosquitoes from glucose solution for 4-5 days to stimulate host-seeking behaviour. Although this deprivation period was longer in comparison with earlier studies (Cornet et al. 2013, Simpson et al. 2009), it did not negatively affect the mosquito's survival. This deprivation may interact with pathogen infection, because pathogens use host resources to develop and replicate (Cressler et al. 2014). However, WNV infected mosquitoes showed similar flight activity compared to both control and mock-infected mosquitoes. Thus, glucose deprivation does not seem to result in a higher energy depletion of WNV infected mosquitoes. For future research, it would be interesting to investigate the effect of long-term glucose deprivation on survival of WNV-infected mosquitoes.

In this study mosquitoes were infected with WNV through intra-thoracic injections. Injections are needed to guarantee that WNV disseminates to the salivary glands of all mosquitoes at 8-10 days post infection (Vogels et al. 2016a). In addition, use of naïve females which have no prior blood-feeding experience, allows for recording of unbiased responses towards host odours in the olfactometer. Our previous study showed that viral titers after an infectious blood meal or injections are within the same range. Results obtained with mock infected mosquitoes showed that the injection procedure itself did not have any effects on mosquito behaviour or fitness. Thus, although injections are not a natural way to infect mosquitoes, we believe that this method does provide results that are relevant for the natural situation.

Future research will focus on the effect of other arboviruses on the host seeking behaviour of mosquitoes, in order to investigate whether this phenomenon is specific for WNV or more broadly occurring with other arbovirus-vector systems. Understanding the influence of arbovirus infection on vector behaviour is necessary to fully understand dynamics of arbovirus transmission, which is needed for proper risk assessment and tailored control strategies.

\section{Conclusions}

WNV infection decreases the mosquito's host-seeking response, and does not induce a shift in host preference towards avian hosts. Other mosquito fitness-related traits (flight activity, blood feeding propensity, and survival) are not affected by WNV infection. The reduced host-seeking response cannot be explained by inhibition of peripheral odour perception via the antennae, but might be due to interference of WNV with neural processing in the mosquito's brain. Our results suggest a mechanism of natural selection favouring intermediate WNV replication due to a dose-dependent trade-off between dissemination to the saliva and infection of the brain. 


\section{Acknowledgements}

We thank Jeroen Spitzen, Niels Verhulst, Olaf Wilting, Lennart van de Peppel, Bram van de Straat, and Steffanie Teekema for assistance during odour collections, Corinne Geertsema for maintenance of cell cultures, Hans Smid for support and training in EAG experiments, and Marcel Dicke for providing comments on an earlier version of this chapter. 



\section{Chapter 7}

\section{Modelling West Nile virus transmission risk in Europe: effect of temperature and mosquito biotypes on the basic reproduction number}

Chantal B.F. Vogels ${ }^{1^{*}}$, Nienke Hartemink ${ }^{2^{*}}$, and Constantianus J.M. Koenraadt ${ }^{1}$

\footnotetext{
* These authors contributed equally to this work

${ }^{1}$ Laboratory of Entomology, Wageningen University \& Research

${ }^{2}$ Theoretical Ecology group, Institute for Biodiversity and Ecosystem Dynamics, University of Amsterdam
}

Scientific Reports (2017) 7: 5022 


\begin{abstract}
West Nile virus (WNV) is a mosquito-borne flavivirus which has caused repeated outbreaks in humans in southern and central Europe, but thus far not in northern Europe. The main mosquito vector for WNV, Culex pipiens, consists of two behaviourally distinct biotypes, pipiens and molestus, which can form hybrids. Differences between biotypes, such as vector competence and host preference, could be important in determining the risk of WNV outbreaks. Risks for WNV establishment can be modelled with basic reproduction number $\left(R_{0}\right)$ models. However, existing $R_{0}$ models have not differentiated between biotypes. The aim of this study was, therefore, to explore the role of temperature-dependent and biotypespecific effects on the risk of WNV establishment in Europe. We developed an $R_{0}$ model with temperature-dependent and biotype-specific parameters, and calculated $R_{0}$ values using the next-generation matrix for several scenarios relevant for Europe. In addition, elasticity analysis was done to investigate the contribution of each biotype to $R_{0}$. Global warming and increased mosquito-to-host ratios can possibly result in more intense WNV circulation in birds and spill-over to humans in northern Europe. Different contributions of the $C x$. pipiens biotypes to $R_{0}$ shows the importance of including biotype-specific parameters in models for reliable WNV risk assessments.
\end{abstract}

Keywords: West Nile virus, Culex pipiens, biotypes, basic reproduction number, $R_{0}$, next-generation matrix, elasticity analysis 


\section{Introduction}

During the past years, the arthropod-borne West Nile virus (WNV; family: Flaviviridae), caused repeated outbreaks in humans in Europe (Calistri et al. 2010a). WNV outbreaks have thus far been limited to central, southern, and eastern Europe (ECDC 2016, Hubalek \& Halouzka 1999, Zeller \& Schuffenecker 2004), which is in contrast to the much more severe WNV outbreaks that occurred throughout North America (Hayes \& Gubler 2006). After the initial introduction of WNV in the United States of America in 1999 (Lanciotti et al. 1999), the virus spread within a few years from the east to the west coast (Hayes et al. 2005). Such spread has not been observed in Europe.

WNV is maintained in a natural transmission cycle between ornithophilic mosquitoes and birds, whereas mammals, including humans, are usually dead-end hosts (Bowen \& Nemeth 2007). Culex (Cx.) pipiens mosquitoes have been identified as one of the most important vectors for WNV, due to their vector competence, feeding preferences, and high abundance during summer (Fonseca et al. 2004, Kilpatrick et al. 2005, Papa et al. 2013). The species Cx. pipiens consists of two morphologically identical biotypes, pipiens and molestus, which show different behaviour (Byrne \& Nichols 1999, Shute 1941). Biotype pipiens has a preference for birds, and is therefore thought to be an important enzootic vector for WNV, whereas the molestus biotype prefers to feed on mammals (Fritz et al. 2015, Osório et al. 2014). Hybrids between both biotypes are considered important bridge vectors that can transmit WNV from birds to humans, due to their more generalist feeding character (Fonseca et al. 2004, Fritz et al. 2015, Kilpatrick et al. 2005).

With regard to the situation in northern Europe, it has been shown that European jackdaws and carrion crows, originating from The Netherlands, are susceptible hosts for WNV (Lim et al. 2015, Lim et al. 2014). Our previous studies showed that northern European Cx. pipiens mosquitoes are competent vectors for WNV, and that temperature is an important limiting factor for WNV transmission (Fros et al. 2015a, Fros et al. 2015b, Vogels et al. 2016a). The presence of susceptible hosts and mosquitoes that under favourable climatic conditions are competent vectors for WNV, suggests that the possibility of WNV transmission in northern Europe cannot be ruled out. Recently, we showed that vector competence of northern European Cx. pipiens biotypes and hybrids is differentially affected by temperature (Vogels et al. 2016a). Thus far, differentiation between the Cx. pipiens biotypes and hybrids has not been taken into account in WNV risk assessments. However, differences between biotypes in terms of vector competence response to temperature and in terms of feeding preferences are likely to affect their vectorial capacity for WNV. Therefore, this information needs to be taken into account when assessing risks of 
WNV transmission in northern Europe.

One way to assess the risk of WNV outbreaks is by calculating the basic reproduction number $\left(R_{0}\right)$. The $R_{0}$ represents the average number of secondary cases that can arise after introduction of one infectious individual in a susceptible population (Diekmann et al. 1990, Dietz 1993). If the average number of secondary cases is higher than one $\left(R_{0}>1\right)$, there is a risk for disease establishment in a certain area. This risk of disease establishment increases with higher numbers of secondary cases. If the number of secondary cases is lower than one $\left(R_{0}<1\right)$, an introduced case may lead to a few new cases, just by chance, but the disease is not expected to establish or cause a large outbreak. Whereas the effect of temperature on the $R_{0}$ for WNV has been investigated (Hartley et al. 2012, Kushmaro et al. 2015), no studies differentiated between the behaviourally distinct $C x$. pipiens biotypes in $R_{0}$ models. Differentiation between biotypes is highly important because differences in vector competence and different host feeding behaviour can strongly impact the outcome of $R_{0}$ models (Fritz et al. 2015, Vogels et al. 2016a). Here, we investigated the effects of both temperature and behavioural differences between the $C x$. pipiens biotypes on $R_{0}$, with a focus on the northern European situation. We developed an $R_{0}$ model with temperature-dependent and biotype-specific parameters that were mostly determined for European mosquitoes. Based on this model, differences in establishment of WNV transmission cycles across Europe can be explored.

\section{Materials and Methods}

\section{Model description}

$R_{0}$ is calculated using the next-generation matrix method (Diekmann \& Heesterbeek 2000, Diekmann et al. 1990). We will first derive the next-generation matrix (NGM) and then describe how we can use it to study different scenarios. Each scenario is based on a different combination of parameter values for four factors: temperature, mosquito-to-host ratio, the fraction of birds in the host population, and the biotype composition of the mosquito population.

First, we need to determine the number of types-at-infection. These types-at-infection are the types of individuals that are involved in transmission and that differ from each other from an epidemiological perspective. In this case, there are four types to be distinguished; three mosquito biotypes (biotype pipiens, biotype molestus, and hybrids) and one (generic) reservoir host type (birds). Non-competent hosts, such as humans and mammals, are not explicitly included in the matrix, as they do not contribute to transmission. However, the fact that non-competent hosts may receive bites by infected mosquitoes and that these bites result in a loss from the perspective 
of the virus, is taken into account (see section: transmission from bird to mosquito).

The NGM is then a four by four matrix and its elements represent the reproduction numbers for all pairs of types. That is, each element of the matrix, $k_{i j}$, gives the number of individuals of type $i$ that are infected by one newly infected individual of type $j$ over the course of its infective lifetime.

We assume that transmission only occurs between birds and mosquitoes, not directly among birds or among mosquitoes. This means that we have to derive three terms for transmission from birds to each of the three biotypes $\left(k_{\mathrm{pb}}, k_{\mathrm{mb}}, k_{\mathrm{hb}}\right)$, where subscripts $\mathrm{p}$ indicates biotype pipiens, $\mathrm{m}$ indicates biotype molestus, $\mathrm{h}$ indicates hybrid, and $\mathrm{b}$ indicates bird. Vice versa, we need three terms to describe transmission from each of the biotypes to birds $\left(k_{\mathrm{bp}}, k_{\mathrm{bm}}, k_{\mathrm{bh}}\right)$. The NGM can then be specified as follows:

$$
\left[\begin{array}{cccc}
0 & 0 & 0 & k_{\mathrm{pb}} \\
0 & 0 & 0 & k_{\mathrm{mb}} \\
0 & 0 & 0 & k_{\mathrm{hb}} \\
k_{\mathrm{bp}} & k_{\mathrm{bm}} & k_{\mathrm{bh}} & 0
\end{array}\right]
$$

Before we derive the terms in the NGM, we here give some notes on the notation. Throughout the manuscript, subscripts are used to indicate temperature- and biotypespecificity of parameters. Subscript $x$ is used to indicate that a parameter value is biotype-specific (but when referring to a specific biotype, we use subscripts $p, m$, and $\mathrm{h}$, as indicated above). Subscript $\mathrm{T}$ indicates that a parameter value is temperaturedependent and the temperature values indicate that it refers to a specific temperature (e.g. $\mathrm{EIP}_{18}$ refers to the duration of the extrinsic incubation period at $18{ }^{\circ} \mathrm{C}$ ).

\section{Transmission from mosquito to bird}

The expected number of birds infected by one newly infected mosquito results from the multiplication of four items:

1. The probability that this newly infected mosquito survives long enough to become infectious, that is, to survive the extrinsic incubation period (EIP). This probability can be estimated in several ways (Brand et al. 2016, Hartemink et al. 2015). Here we assume that a vector can only become infectious after completion of the EIP, hence the daily survival probability $(p)$ is raised to the power of the duration of the EIP. Both $p$ and EIP are temperature-dependent (hence they are indicated by $p_{\mathrm{T}}$ and $\mathrm{EIP}_{\mathrm{T}}$ ).

2. The number of bites per day that this mosquito takes on birds after becoming 
infectious, which is a multiplication of the temperature-dependent biting rate $a_{\mathrm{T}}$ (the number of bites per day, or the reciprocal of the number of days between blood meals), and the probability $\left(\phi_{x}\right)$ that a bite will be on a bird. The modelling of the biotype-specific host preference is described below (section: biotypespecific host preferences $\phi_{\mathrm{x}}$ ).

3. The probability per bite to transmit the virus $(b)$.

4. The duration of the infectious period of the mosquito, which is the expected remaining life span of the mosquito $(1 /-\ln (p))$.

The expected number of birds, infected by one newly infected mosquito is then:

$$
k_{\mathrm{bx}}=\frac{\phi_{\mathrm{x}}{ }^{*} a_{\mathrm{T}}{ }^{*} b^{*} p_{\mathrm{T}}^{\mathrm{EIP}}}{\left(-\ln \left(p_{\mathrm{T}}\right)\right)}
$$

where $a$, EIP and $p$ depend on temperature (Table 1).

Table 1: Parameter estimates for basic reproduction number $\left(\mathbf{R}_{0}\right)$ model

\begin{tabular}{|c|c|c|c|}
\hline Parameter & Description & $\begin{array}{l}\text { Point estimates } \\
\text { (range) }\end{array}$ & References \\
\hline$a_{18}$ & Daily biting rate at $18^{\circ} \mathrm{C}$ & $0.14(0.12-0.16)$ & $\begin{array}{l}\text { (Faraj et al. 2006, } \\
\text { Platonov et al. 2008) }\end{array}$ \\
\hline$a_{23}$ & Daily biting rate at $23^{\circ} \mathrm{C}$ & $0.17(0.15-0.19)$ & $\begin{array}{l}\text { (Faraj et al. 2006, } \\
\text { Platonov et al. 2008) }\end{array}$ \\
\hline$a_{28}$ & Daily biting rate at $28^{\circ} \mathrm{C}$ & $0.20(0.17-0.23)$ & $\begin{array}{l}\text { (Faraj et al. 2006, } \\
\text { Platonov et al. 2008) }\end{array}$ \\
\hline$b$ & Transmission probability mosquito to bird & 0.80 & $\begin{array}{l}\text { (Hartemink et al. 2007, } \\
\text { Kushmaro et al. 2015) }\end{array}$ \\
\hline$c_{\mathrm{p} 18}$ & $\begin{array}{l}\text { Transmission probability bird to pipiens } \\
\text { at } 18^{\circ} \mathrm{C}\end{array}$ & $0.04(0.00-0.07)$ & (Vogels et al. 2016a) \\
\hline$c_{\mathrm{p} 23}$ & $\begin{array}{l}\text { Transmission probability bird to pipiens } \\
\text { at } 23^{\circ} \mathrm{C}\end{array}$ & $0.09(0.01-0.17)$ & (Vogels et al. 2016a) \\
\hline$c_{\mathrm{p} 28}$ & $\begin{array}{l}\text { Transmission probability bird to pipiens } \\
\text { at } 28^{\circ} \mathrm{C}\end{array}$ & $0.34(0.20-0.48)$ & (Vogels et al. 2016a) \\
\hline$c_{\mathrm{m} 18}$ & $\begin{array}{l}\text { Transmission probability bird to molestus } \\
\text { at } 18^{\circ} \mathrm{C}\end{array}$ & $0.09(0.01-0.17)$ & (Vogels et al. 2016a) \\
\hline$c_{\mathrm{m} 23}$ & $\begin{array}{l}\text { Transmission probability bird to molestus } \\
\text { at } 23^{\circ} \mathrm{C}\end{array}$ & $0.13(0.03-0.22)$ & (Vogels et al. 2016a) \\
\hline$c_{\mathrm{m} 28}$ & $\begin{array}{l}\text { Transmission probability bird to molestus } \\
\text { at } 28^{\circ} \mathrm{C}\end{array}$ & $0.13(0.03-0.22)$ & (Vogels et al. 2016a) \\
\hline$c_{\mathrm{h} 18}$ & $\begin{array}{l}\text { Transmission probability bird to hybrid } \\
\text { at } 18^{\circ} \mathrm{C}\end{array}$ & $0.05(0.00-0.11)$ & (Vogels et al. 2016a) \\
\hline
\end{tabular}




\begin{tabular}{|c|c|c|c|}
\hline$c_{\mathrm{h} 23}$ & $\begin{array}{l}\text { Transmission probability bird to hybrid } \\
\text { at } 23^{\circ} \mathrm{C}\end{array}$ & $0.04(0.00-0.07)$ & (Vogels et al. 2016a) \\
\hline$C_{\mathrm{h} 28}$ & $\begin{array}{l}\text { Transmission probability bird to hybrid } \\
\text { at } 28^{\circ} \mathrm{C}\end{array}$ & $0.17(0.06-0.27)$ & (Vogels et al. 2016a) \\
\hline $1 / r_{b}$ & Number of days a bird remains infectious & 5.50 & $\begin{array}{l}\text { (Lim et al. 2015, Lim et } \\
\text { al. 2014) }\end{array}$ \\
\hline $\mathrm{EIP}_{18}$ & $\begin{array}{l}\text { Extrinsic incubation period at } 18^{\circ} \mathrm{C} \\
\text { (days) }\end{array}$ & $15(12-18)$ & (Kilpatrick et al. 2008) \\
\hline $\mathrm{EIP}_{23}$ & $\begin{array}{l}\text { Extrinsic incubation period at } 23^{\circ} \mathrm{C} \\
\text { (days) }\end{array}$ & $9(7-11)$ & $\begin{array}{l}\text { (Fros et al. 2015a, } \\
\text { Kilpatrick et al. 2008) }\end{array}$ \\
\hline $\mathrm{EIP}_{28}$ & $\begin{array}{l}\text { Extrinsic incubation period at } 28^{\circ} \mathrm{C} \\
\text { (days) }\end{array}$ & $4(3-5)$ & (Kilpatrick et al. 2008) \\
\hline$p_{18}$ & Daily survival probability at $18^{\circ} \mathrm{C}$ & $0.97(0.96-0.98)$ & $\begin{array}{l}\text { (Ciota et al. 2014, } \\
\text { Ruybal et al. 2016) }\end{array}$ \\
\hline$p_{23}$ & Daily survival probability at $23^{\circ} \mathrm{C}$ & $0.96(0.95-0.97)$ & $\begin{array}{l}\text { (Ciota et al. 2014, } \\
\text { Ruybal et al. 2016) }\end{array}$ \\
\hline$p_{28}$ & Daily survival probability at $28^{\circ} \mathrm{C}$ & $0.95(0.94-0.96)$ & $\begin{array}{l}\text { (Ciota et al. 2014, } \\
\text { Ruybal et al. 2016) }\end{array}$ \\
\hline$\phi_{\mathrm{p}}$ & Host preference pipiens & $1-(1-F B)^{2.75}$ & $\begin{array}{l}\text { (Fritz et al. 2015, } \\
\text { Osório et al. 2014) }\end{array}$ \\
\hline$\phi_{\mathrm{m}}$ & Host preference molestus & $\mathrm{FB}^{2.75}$ & $\begin{array}{l}\text { (Fritz et al. 2015, } \\
\text { Osório et al. 2014) }\end{array}$ \\
\hline$\phi_{\mathrm{h}}$ & Host preference hybrid & FB & $\begin{array}{l}\text { (Fritz et al. 2015, } \\
\text { Osório et al. 2014) }\end{array}$ \\
\hline $\mathrm{T}$ & Temperature $\left({ }^{\circ} \mathrm{C}\right)$ & 18,23, or 28 & \\
\hline C & Mosquito-to-host ratio & 10,100 , or 250 & $\begin{array}{l}\text { (Balenghien et al. } \\
\text { 2006) }\end{array}$ \\
\hline FB & Fraction of birds in the host population & 0.2 or 0.8 & \\
\hline$m_{\mathrm{p}}$ & Fraction pipiens in the population & $0.10,0.33$, or 0.80 & $\begin{array}{l}\text { (Di Luca et al. 2016, } \\
\text { Vogels et al. 2016b) }\end{array}$ \\
\hline$m_{\mathrm{m}}$ & Fraction molestus in the population & $0.10,0.33$, or 0.80 & $\begin{array}{l}\text { (Di Luca et al. 2016, } \\
\text { Vogels et al. 2016b) }\end{array}$ \\
\hline$m_{\mathrm{h}}$ & Fraction hybrids in the population & 0.10 or 0.33 & $\begin{array}{l}\text { (Di Luca et al. 2016, } \\
\text { Vogels et al. 2016b) }\end{array}$ \\
\hline
\end{tabular}




\section{Biotype-specific host preferences $\left(\phi_{\mathrm{x}}\right)$}

Each biotype has a different host preference that needs to be taken into account in the model. For each of the biotypes, we derive a simple algorithm that describes how $\phi_{\mathrm{x}}$, the probability that a blood meal is taken on a bird, varies with the fraction of birds in the total host population (Table 1 and Figure 1). We assume that when preferred hosts are not available, mosquitoes will feed on a non-preferred host, rather than not feeding at all. Experimental data indicate that when given a choice between a bird and a mammal, the probability that the mosquito will choose the bird, is approximately $85 \%$ for the pipiens biotype, $15 \%$ for the molestus biotype, and $50 \%$ for the hybrid (Fritz et al. 2015). For all biotypes, the probability to feed on a bird when there are no birds should be zero (fraction of birds in the host population $(F B)=0$ ), and the probability is one when there are only birds $(F B=1)$. For biotype pipiens, the function that describes the relation between $\mathrm{FB}$ and $\phi_{\mathrm{p}}$ should be convex. At equal fractions of birds and other hosts (FB $=0.5)$, the probability should be approximately $85 \%$. For biotype molestus, the function is concave, with a probability to bite a bird of 15 $\%$ at equal fractions of birds and other hosts ( $F B=0.5)$. For hybrids, the function is a straight line, so that the probability to feed on a bird increases linearly with the fraction of available hosts that is a bird (Figure 1).

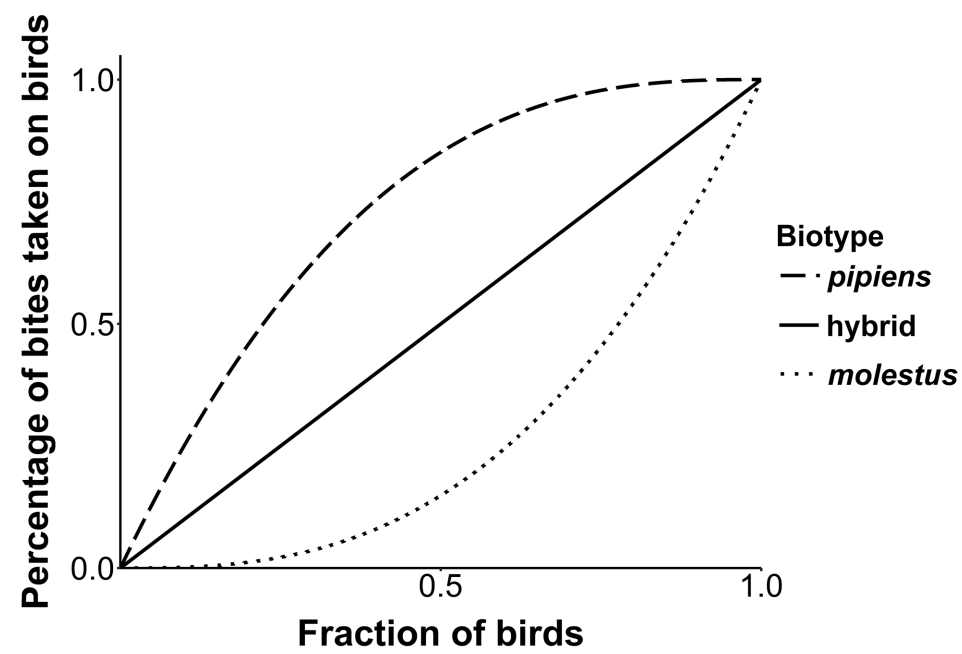

Figure 1: Relation between fraction of birds in the host population and the percentage of bites taken on birds. Percentage of mosquito bites taken on birds by biotype pipiens, biotype molestus, and hybrids, as a function of the fraction of bird hosts in the total host population. 


\section{Transmission from bird to mosquito}

The expected number of mosquitoes of a certain biotype infected by one newly infected bird results from the multiplication of:

1. The number of bites that this bird receives from this particular biotype. This number depends on the abundance of the mosquito biotype, its host preference, the number of birds and other hosts, and the temperature-dependent biting rate $a_{T}$. Imagine a local mosquito population of $X$ individuals. Of these $X$ mosquitoes, $a_{\mathrm{T}}{ }^{*} X$ will be searching for a blood meal on a particular day. Multiplying these $a_{\mathrm{T}}{ }^{*} X$ individuals with the fraction of mosquitoes that is of the specific biotype $\left(m_{\mathrm{p}}, m_{\mathrm{m}}\right.$ and $m_{\mathrm{h}}$, for the fractions pipiens, molestus and hybrid, respectively) and with their respective host preferences $\phi_{\mathrm{p}}, \phi_{\mathrm{m}}$, and $\phi_{\mathrm{h}}$ gives the number of bites by a certain biotype per bird:

$$
\left(\phi_{\mathrm{x}}{ }^{*} a_{\mathrm{T}}{ }^{*} m_{\mathrm{x}}{ }^{*} X\right) / \text { bird }
$$

Rather than varying the numbers of mosquitoes, birds and alternative hosts, which would require varying three factors, we prefer to work with two ratios: the mosquito-to-host ratio $(C)$, and the fraction of the host population that consists of birds (FB). If the total number of hosts is $Y$, then the number of birds is $\mathrm{FB}^{*} Y$. The mosquito-to-host ratio $C$ is equal to $X / Y$, so the mosquito-to-bird ratio is $X I$ $\left(F^{*} Y\right)=C / F B$. The term for the number of bites of mosquitoes of biotype $x$ on birds then becomes:

$$
\phi_{\mathrm{x}}{ }^{*} a_{\mathrm{T}}{ }^{*} m_{\mathrm{x}}{ }^{*} \mathrm{C} / \mathrm{FB}
$$

For our model, we selected three values for mosquito-to-host ratio based on results of trap catches (Almeida et al. 2008, Balenghien et al. 2006), which reflect areas with low, intermediate, and high mosquito-to-host ratios $(C=10$, 100 , and 250). In addition, we analysed two values for bird fraction in the host population: low $(F B=0.2)$ and high bird fraction ( $F B=0.8)$.

2. The biotype- and temperature-specific probability of transmission from bird to mosquito per bite, denoted by $c_{\mathrm{x} T}$.

3. The expected duration of the infectious period of a bird, which is modelled as the reciprocal of the recovery rate $r_{\mathrm{b}}$.

The term for the number of mosquitoes of biotype $x$ that is infected by a newly infected bird is then as follows:

$$
k_{\mathrm{xb}}=\frac{\phi_{\mathrm{x}}{ }^{*} a_{\mathrm{T}}{ }^{*} C_{\mathrm{xT}}{ }^{*} m_{\mathrm{x}}{ }^{*} \mathrm{C} / \mathrm{FB}}{r_{\mathrm{b}}}
$$




\section{Biotype-specific and temperature-dependent transmission efficiency $\left(c_{\mathrm{xT}}\right)$}

The probability that a mosquito establishes an infection after feeding on an infected bird generally increases with temperature. However, our earlier work demonstrated that the relationship between temperature and transmission probability is different for each of the three biotypes (Vogels et al. 2016a). We, therefore, used the results of our laboratory experiments on the vector competence of the three biotypes at $18{ }^{\circ} \mathrm{C}, 23{ }^{\circ} \mathrm{C}$, and $28{ }^{\circ} \mathrm{C}$ (Vogels et al. 2016a). For each biotype, we calculated the confidence interval of the observed values for the vector competence at each temperature (Table 1).

\section{Scenarios}

Based on the terms derived above for the transmission for each of the pairs in the matrix, the NGM now looks as follows:

$$
\left[\begin{array}{cccc}
0 & 0 & 0 & \frac{\phi_{\mathrm{p}}{ }^{*} a_{\mathrm{T}}{ }^{*} c_{\mathrm{pT}}{ }^{*} m_{\mathrm{p}}{ }^{*} C / \mathrm{FB}}{r_{\mathrm{b}}} \\
0 & 0 & 0 & \frac{\phi_{\mathrm{m}}{ }^{*} a_{\mathrm{T}}{ }^{*} c_{\mathrm{mT}}{ }^{*} m_{\mathrm{m}}{ }^{*} C / \mathrm{FB}}{r_{\mathrm{b}}} \\
\frac{\phi_{\mathrm{p}}{ }^{*} a_{\mathrm{T}}{ }^{*} b^{*} p_{\mathrm{T}}^{\mathrm{EIPT}}}{\left(-\ln \left(p_{\mathrm{T}}\right)\right)} & \frac{\phi_{\mathrm{m}}{ }^{*} a_{\mathrm{T}}{ }^{*} b^{*} p_{\mathrm{T}}^{\mathrm{EIP}}}{\left(-\ln \left(p_{\mathrm{T}}\right)\right)} & \frac{\phi_{\mathrm{h}}{ }^{*} a_{\mathrm{T}}{ }^{*} b^{*} p_{\mathrm{T}}^{\mathrm{EIPT}}}{\left(-\ln \left(p_{\mathrm{T}}\right)\right)} & \frac{\phi_{\mathrm{h}}{ }^{*} a_{\mathrm{T}}{ }^{*} c_{\mathrm{hT}}{ }^{*} m_{\mathrm{h}}{ }^{*} C / \mathrm{FB}}{r_{\mathrm{b}}}
\end{array}\right]
$$

The value for $R_{0}$ can then be calculated for different parameterizations of the NGM. For this purpose, we used the popbio package in $\mathrm{R}$ to calculate the eigenvalue of the matrix, which represents the $R_{0}$ estimate (R Core Team 2016). We calculated $R_{0}$ based on the parameter ranges given in Table 1 , but with different values for:

1. Temperature $\left(\mathrm{T}=18{ }^{\circ} \mathrm{C}, 23^{\circ} \mathrm{C}\right.$, and $\left.28^{\circ} \mathrm{C}\right)$,

2. Mosquito-to-host ratio ( $C=10,100$, and 250; Almeida et al. 2008, Balenghien et al. 2006),

3. Fraction of hosts that is a bird ( $F B=0.2$ and 0.8$)$,

4. The biotype composition of the mosquito population, which has been shown to be highly variable across different locations in Europe (Di Luca et al. 2016, Vogels et al. 2016b). To express the variability we selected three representative biotype compositions; either dominated by pipiens $\left(m_{\mathrm{p}}=0.8, m_{\mathrm{m}}=0.1\right.$, and $m_{\mathrm{h}}=$ $0.1)$, dominated by molestus $\left(m_{\mathrm{p}}=0.1, m_{\mathrm{m}}=0.8\right.$, and $m_{\mathrm{h}}=0.1$ ) or equal ratios of the three biotypes $\left(m_{\mathrm{p}}=0.33, m_{\mathrm{m}}=0.33\right.$, and $\left.m_{\mathrm{h}}=0.33\right)$. 
$R_{0}$ values were calculated for each combination of the above-mentioned parameter values, so for a total of $\left(3^{*} 3^{*} 2^{*} 3=\right) 54$ different scenarios. For each scenario, we calculated $100,000 R_{0}$ values based on uniform sampling from the parameter value ranges (Table 1). These 100,000 values were then used to calculate the mean $R_{0}$ values for each scenario. For each scenario this gives a range of $R_{0}$ values rather than a single point estimate, and this range reflects the uncertainty in the parameter estimates.

When interpreting the results, it should be noted that not all scenarios are equally likely to occur, as mosquito biotypes are likely to live in environments where their preferred hosts are found. We hypothesize that dominance of the pipiens biotype is most likely to occur in environments where birds are the most abundant hosts (i.e. FB is high) and not very likely in an environment with mostly other hosts (i.e. a low FB). In the case of a low fraction of birds, we expect a higher fraction of biotype molestus. Hence, we think that scenarios that involve a combination of a high FB and a low fraction of biotype pipiens or a low FB and high fraction of pipiens are not representative of a real situation. Therefore, we decided to present the 54 scenarios in six plots (with low and high FB and with the three mosquito population compositions). For each of these groups of scenarios, the estimated value for $R_{0}$ is given for each possible combination of temperature and mosquito-to-host ratio $C$.

In addition to $R_{0}$ calculations, elasticity analysis can be done on the NGM. By applying this analysis, which is a form of perturbation analysis, to the elements of the NGM, the contribution of each of the biotypes to the total can be quantified. This method has been applied in population biology for a long time (de Kroon et al. 1986), but more recently, it has been used in vector-borne disease eco-epidemiology (Hartemink et al. 2008, Matser et al. 2009). For this analysis one calculates the proportional response in a dependent variable resulting from a proportional perturbation in an independent variable (Caswell 2001). The interesting aspect for our application is that the elasticities of the dominant eigenvalue of a matrix to the matrix elements always sum to one (Mesterton-Gibbons 1993). In population biology, this has led to their interpretation as the relative contribution to population growth from the respective transition in the life cycle (de Kroon et al. 1986). Similarly, the element elasticities of an NGM can be interpreted as contributions to $R_{0}$. The relative contributions are given exactly by the elasticities. Composite elasticities, sums of particular sets of element elasticities, follow from the interpretation of element elasticities as contributions to population growth rate or $R_{0}$ (Hartemink et al. 2008, Matser et al. 2009). In this case, the two elements that refer to a specific biotype will always be the same. We, therefore, chose to plot the proportion of the transmission associated with each of the biotypes. Calculations of $R_{0}$ were done based on the point estimates for each parameter (Table 1). 


\section{Results}

In total, 54 scenarios were modelled in order to investigate the effects of temperature, bird fraction, mosquito-to-host ratio, and $C x$. pipiens biotype composition on $R_{0}$ (Figure 2). The first observation is that there are considerable differences among the 54 different mean values of $R_{0}$, ranging from values well below one to values of $R_{0}$ of approximately fifteen. Temperature, mosquito-to-host ratio $(C)$, population composition of hosts (bird fraction FB), and biotype composition are all important in determining the value of $R_{0}$. Here we will describe the effects for each of the variables.

\section{Temperature}

Within each of the six plots, $R_{0}$ values increased with higher temperatures (Figure 2). Our results suggest that under favourable climatic conditions with relatively high average temperatures of at least $28^{\circ} \mathrm{C}$, there is a high chance that WNV transmission cycles become established. Such temperatures are typical for southern European countries. The risk of WNV establishment seems, therefore, highly dependent on temperature and to a much lesser extent dependent on the exact mosquito population composition, bird fraction, and mosquito-to-host ratio. In contrast, $R_{0}$ values were much lower at $18{ }^{\circ} \mathrm{C}$, especially for scenarios with the lowest mosquito-to-host ratio $(C=10)$. This suggests that WNV establishment in regions with low average summer temperatures of $18{ }^{\circ} \mathrm{C}$, such as northern Europe, seems only possible when a high number of mosquitoes is present per host. This confirms that temperature is one of the most important drivers for WNV transmission (Paz et al. 2013), which can explain the differences in WNV transmission between northern and southern Europe.

\section{Mosquito-to-host ratio}

In all six plots the $R_{0}$ values increased when more mosquitoes are available per host (Figure 2). Regions with on average at least 100 mosquitoes available per host, have an increased risk of WNV establishment because of relatively high $R_{0}$ values at all temperatures. Thus, the mosquito-to-host ratio is a second important determinant for the risk of WNV establishment in a certain area. 

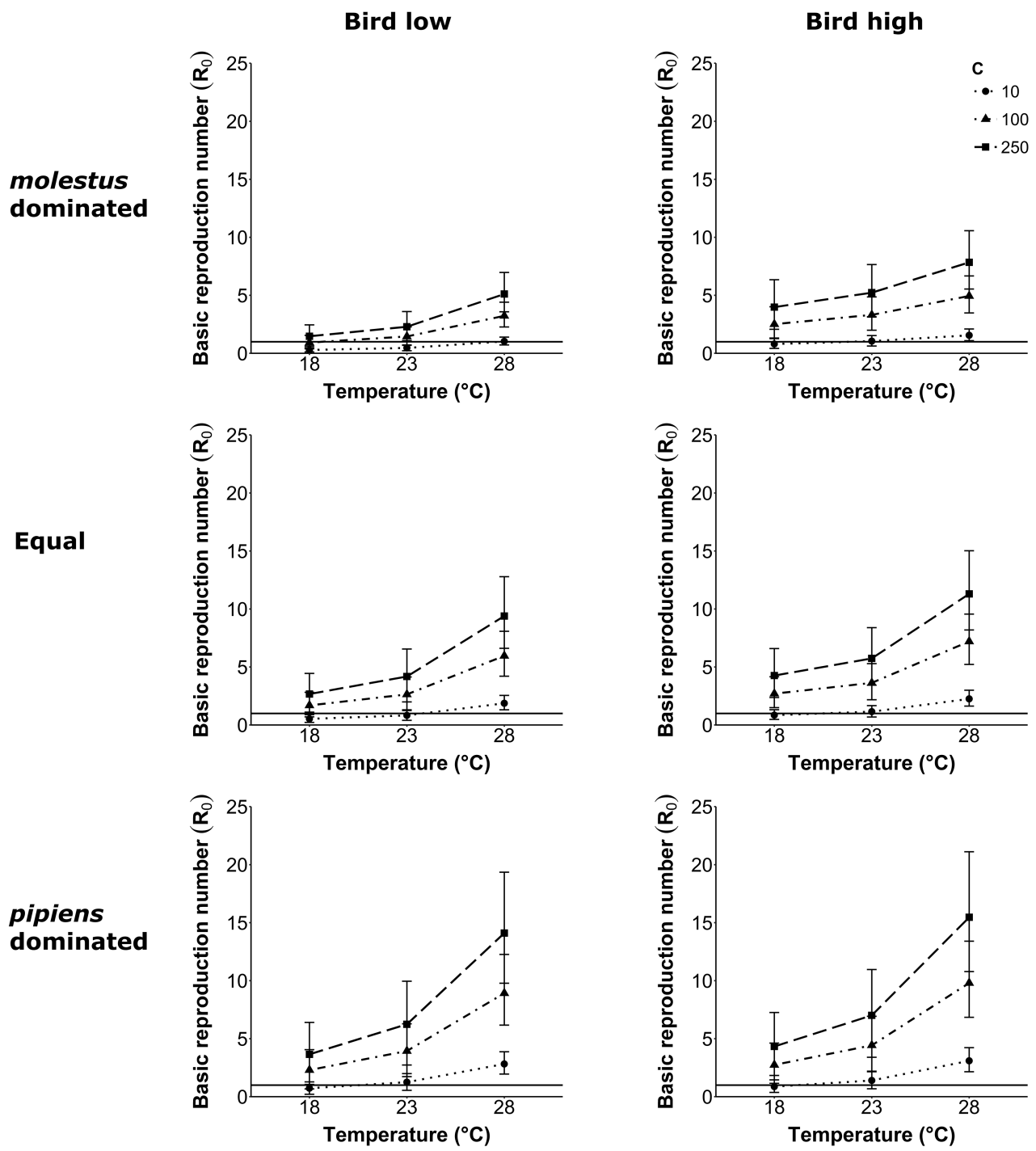

Figure 2: Scenarios for West Nile virus transmission risk modelled with parameters relevant for Europe. The six plots show the results for low or high fractions of birds in the host population $(F B=0.2$ and $\mathrm{FB}=0.8$, respectively) and one of the three different mosquito population compositions (molestus dominated, equal fractions, or pipiens dominated). Each plot shows the mean $R_{0}$ out of 100,000 model calculations, with error bars showing the $95 \%$ range of calculated $R_{0}$ values for three temperature scenarios $\left(\mathrm{T}=18{ }^{\circ} \mathrm{C}, 23^{\circ} \mathrm{C}\right.$, and $\left.28^{\circ} \mathrm{C}\right)$ and three different mosquito-to-host ratios $(C=10,100$, or 250$)$. The horizontal line indicates an $R_{0}$ value of one. Values of $R_{0}$ above one indicate that there is a chance of West Nile virus establishment. 


\section{Fraction of birds}

The difference in $R_{0}$ values between the main scenarios with low and high bird fraction is relatively small (Figure 2). This is linked to the fact that fraction of birds in the host population determines both the host preference of the biotypes as well as the distribution of the bites over the birds. High fractions of birds mean that all biotype pipiens and also a substantial part of the molestus and hybrid mosquitoes will feed on birds, but also that the bites will be distributed over more birds. Thus, the actual number of bites per bird decreases with higher fraction of birds, which results in fewer mosquitoes infected by one bird. However, the number of birds infected by one mosquito increases, as the preference shifts to birds with increasing FB. These two effects counteract each other, but overall, the $R_{0}$ was slightly higher for the scenarios with high FB.

\section{Biotype composition}

Biotype pipiens plays an important role in WNV transmission due to its preference for birds and relatively high vector competence, especially at $28^{\circ} \mathrm{C}$. With increasing fractions of biotype pipiens in the population, there is an increase in $R_{0}$ values for all scenarios (Figure 2). In the main scenarios with equal fractions of biotypes and their hybrids, there is also a relatively high risk of WNV establishment, especially when mosquito-to-host ratios are at a medium or high level. In such scenarios, there is a higher chance of spill-over to humans if WNV transmission can get established.

\section{Biotype contribution}

In order to investigate the role of each Cx. pipiens biotype in WNV transmission, we performed elasiticity analyses to calculate the contribution of each biotype to the mean $R_{0}$ (Figure 3). At low bird fractions, biotype molestus and hybrids will mainly bite hosts other than birds, whereas biotype pipiens will still have a preference for birds. Therefore, in all three main scenarios with a low bird fraction, biotype pipiens is mainly responsible for WNV transmission. In contrast, when the fraction of birds increases the contributions of biotype molestus and hybrids also increase due to a shift in host acceptance towards birds (Figure 1). In mosquito populations with high fractions of biotype molestus, they are the main contributor to $R_{0}$ (Figure 3). However, scenarios with both a high fraction of birds and a high fraction of biotype molestus are unlikely to occur in natural environments. Overall, hybrids may play a minor role in the initial phase of WNV transmission, but may become more important once WNV transmission cycles have established in a certain area. Presence of hybrids increases the risk of infection of humans with WNV. 
Bird low
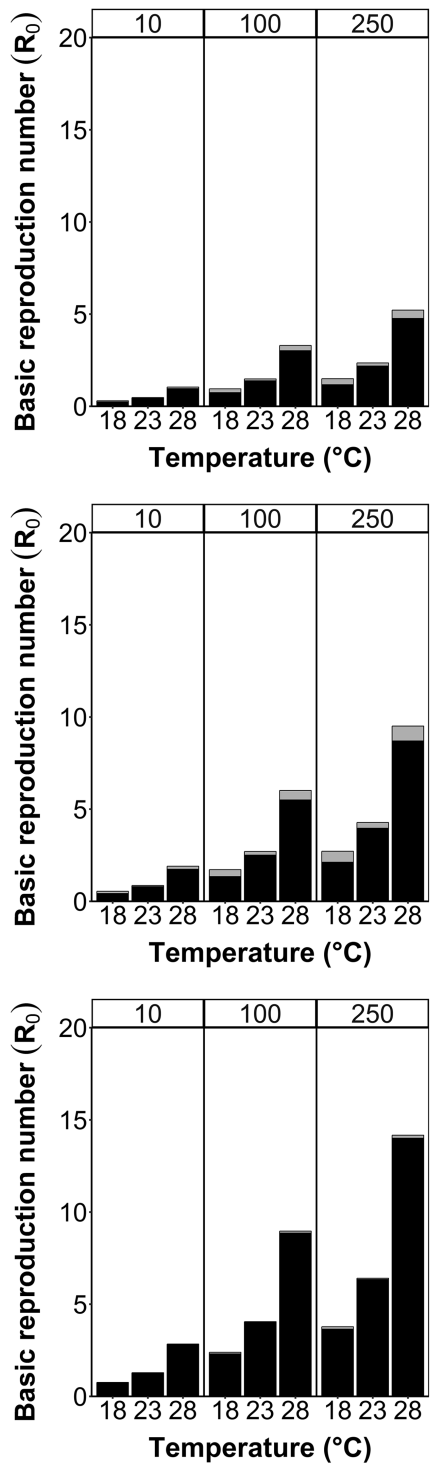

Bird high
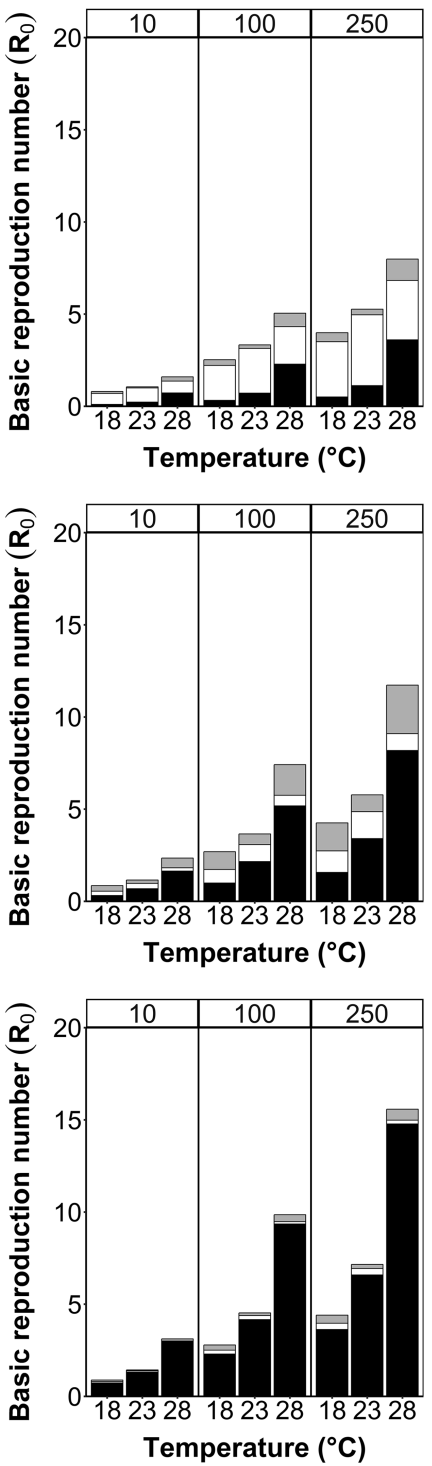

pipiens
dominated

Cx. p. pipiens

Cx. p. molestus

Hybrid

Figure 3: Contribution of each Culex pipiens biotype or hybrid to scenarios for West Nile virus transmission in Europe. The six plots show the results for low or high fractions of birds in the host population ( $\mathrm{FB}=0.2$ and $\mathrm{FB}=0.8$, respectively) and one of the three different mosquito population compositions (molestus dominated, equal fractions, or pipiens dominated). Each plot shows the contribution of biotype pipiens, biotype molestus or hybrids to the $R_{0}$ values for three temperature scenarios $\left(T=18{ }^{\circ} \mathrm{C}, 23^{\circ} \mathrm{C}\right.$, and $\left.28^{\circ} \mathrm{C}\right)$ and three different mosquito-to-host ratios $(C=10,100$, or 250$)$. Values of $R_{0}$ above one indicate that there is a chance of West Nile virus establishment. 


\section{Discussion}

The aim of this study was to explore the role of temperature-dependent and mosquito biotype-specific effects on the risk of WNV establishment, using laboratory and field-derived data. We show that various sets of parameters, comprising 54 scenarios, result in distinct $R_{0}$ outcomes, which clearly demonstrates the complexity of the different parameters modelled in this study. Especially the interaction between temperature and biotype composition, and the mosquito-to-host ratio are main factors that influence $R_{0}$, and, thus, the chance that WNV can get established in a certain area. In addition, $C x$. pipiens biotypes contributed differentially to the $R_{0}$ which underscores the necessity to include biotype-specific parameters in models for reliable WNV risk assessments.

Temperature does not only have a direct effect on viruses (e.g. increased viral replication at higher temperatures (Chamberlain \& Sudia 1961, Hardy et al. 1983)), but it also affects mosquitoes in several ways, such as decreased duration of the gonotrophic cycle, and increased biting rate at higher temperatures (Ciota et al. 2014, Platonov et al. 2008). These effects together result in higher vector competence for WNV at higher temperatures (Fros et al. 2015a, Vogels et al. 2016a), which, in its turn, contributes to a higher risk of WNV establishment (Paz \& Semenza 2013). In this study, we chose representative average summer temperatures for northern $\left(18{ }^{\circ} \mathrm{C}\right)$ and southern Europe $\left(28{ }^{\circ} \mathrm{C}\right)$, as well as an intermediate temperature (23 ${ }^{\circ} \mathrm{C}$; Haylock et al. 2008). In all scenarios, temperatures of $28{ }^{\circ} \mathrm{C}$ allowed for establishment of WNV, which is in line with ongoing WNV circulation and outbreaks in European countries such as Italy, Hungary, and Greece (Chancey et al. 2015, Fros et al. 2015a). In contrast, WNV establishment is only likely at temperatures of $18^{\circ} \mathrm{C}$, if there is a high mosquito-to-host ratio. This shows that temperature is an important limiting factor for WNV transmission in northern Europe. More frequent and longer periods of intense heat in northern Europe due to global warming may, thus, coincide with establishment of WNV transmission cycles in northern Europe (Beniston et al. 2007, Meehl \& Tebaldi 2004, Paz 2015, Semenza et al. 2016).

WNV transmission risk is not only influenced by temperature, but also by several other environmental factors, such as rainfall and humidity (Paz 2015, Shaman \& Day 2007, Shaman et al. 2005). Depending on local circumstances, rainfall and humidity can have positive as well as negative effects on WNV transmission risk (Hahn et al. 2015, Koenraadt \& Harrington 2008, Paz et al. 2013). Therefore, these effects are difficult to incorporate in the model. However, since most of the impact of rainfall and humidity will be via their effects on mosquito population density, we can consider these effects indirectly by looking at the mosquito-to-host ratio $C$. By considering different values for this parameter, we have indirectly taken different scenarios for 
favourable (high $C$ ) and less favourable circumstances (low $C$ ) for mosquitoes in terms of rainfall and humidity into account.

The rationale and assumptions underlying our model are similar to the ones of the traditional Ross-Macdonald model equation (Garrett-Jones \& Shidrawi 1969, Macdonald 1957, Smith et al. 2012). The main difference is that the next-generation approach allows us to include multiple types of infectious individuals, in this case different biotypes. The EIP is assumed to take a fixed number of days and the biting and mortality rates are constant (that is, the times to the next bite and to death are exponentially distributed). This set of assumptions, with a fixed duration for the EIP, might give a lower survival of the EIP than other assumptions (Brand et al. 2016), which means that our estimates for $R_{0}$ are likely to be conservative. However, as we selected a broad range of parameter in diverse scenarios, the overall range in $R_{0}$ values is still relatively large.

In our model, we did not include vertical transmission from mosquito to mosquito, or bird to bird. However, vertical transmission may contribute to WNV transmission in the field, and can especially be key for overwintering of WNV in vertically infected mosquitoes (Anderson \& Main 2006, Anderson et al. 2012). Consequently, outcomes of the $R_{0}$ may slightly increase if vertical transmission would be included.

The mosquito-to-host ratio is an important determinant of the value of $R_{0}$ (Hartemink et al. 2011, Hartemink et al. 2009). The underlying assumption for including the mosquito-to-host ratio in the $R_{0}$ formula is that the mosquitoes in a certain area will distribute their bites over the hosts available in that area and that the mosquito-to-host ratio thus determines the number of bites that a host will receive. The number of bites received by a host is assumed to be the number of mosquitoes searching for a blood meal (number of mosquitoes multiplied by the probability they will feed on a certain day) divided by the number of hosts. This assumption is perfectly plausible in a small confined space: the mosquito bites are likely to be distributed over the available hosts. However, caution should be taken when modelling host and vector abundance separately, or when varying the mosquito-to-host ratio, as we did in this study by varying the value of $C$. The consequence of this assumption is that the $R_{0}$ estimate decreases with higher host densities (more hosts, so fewer bites per host, resulting in lower $R_{0}$ values). This is counterintuitive and in most cases probably incorrect, since densities of vectors and hosts are not independent. Since vectors depend on hosts for blood meals it is unlikely that high abundances of vectors can persist in an area with few hosts, or vice versa that areas with many hosts would harbour only few vectors. Also, as distance between hosts is not taken into account, the fact that lower host densities actually may hamper the spread or reduce the chance to find another host, is not taken into account. This issue explains why the fraction of birds did not 
seem to affect the risk of WNV transmission in our models: at low values of FB, many pipiens would already bite on birds and at higher values of $F B$, the increase in number of bites would not be very substantial. But at a high FB, the number of birds would be higher, and hence the number of bites would be 'spread out' over more birds, which would lead to fewer bites per bird and hence fewer opportunities for a bird to pass on the infection. As stated above, this assumption may not always be realistic, as vector and host densities are not independent. We tried to at least partly overcome this issue by focusing on the more plausible scenarios in terms of the fractions of birds and the mosquito population. We also refrain from drawing strong conclusions on the effect of varying the fraction of birds or the mosquito-host-ratio itself, as these two variables, together with the mosquito population composition and the host preference, are strongly interacting in the way they determine the number of bites per bird. We, therefore, argue that field studies focussing on the relationship between these variables and the number of bites received by individual hosts would be very important in improving the assessment of risk of mosquito-borne diseases.

The elasticity analysis showed that biotypes contributed very differently to $R_{0}$. These differences are due to the relative abundance of biotypes, biotype-specific behaviour (especially host preference (Fritz et al. 2015)), and vector competence (Vogels et al. 2016a). Biotype pipiens plays an important role in establishment of WNV transmission, due to its preference for birds and relatively high vector competence at $28{ }^{\circ} \mathrm{C}$. Biotype molestus and hybrids contribute remarkably less to WNV establishment, especially in areas with low bird occurrence, because they will readily feed on the more abundant mammalian hosts. However, a shift in feeding behaviour has been observed for biotype molestus in areas with high bird abundance (Gomes et al. 2013b). Such shifting feeding behaviour would increase the contribution of both biotype molestus and hybrids to WNV transmission. More importantly, feeding on both birds and mammals increases the risk that WNV will be bridged from birds to humans and horses. Thus, differentiating between biotypes and including relevant parameters in $R_{0}$ models is essential for accurate WNV risks assessments.

$R_{0}$ models are a powerful tool to assess risks of disease establishment in certain areas. The next-generation matrix approach allows for inclusion of different typeat-infections and here, we used this property to develop a novel $R_{0}$ model that incorporates the different $C x$. pipiens biotypes, their feeding preferences and different response of their vector competence to temperature. We have shown the importance of including these temperature-dependent and biotype-specific effects as the $R_{0}$ values varied substantially between the different scenarios. This stresses the importance of biotype differentiation in entomological surveillance programmes, and of including temperature and biotype-specific parameters in risk assessments. 


\section{Conclusions}

The interaction between temperature and the $C x$. pipiens biotypes, and the mosquitoto-host ratio are important factors that determine the chances of WNV establishment in a certain region. Currently, temperature is an important limiting factor for WNV circulation in northern Europe, but the modelled scenarios show that transmission cannot be ruled out. Accurate data are needed on how the number of bites received by birds varies with mosquito and host abundances, and on how these abundances may vary across habitats. Different contributions of the $C x$. pipiens biotypes to the $R_{0}$ highlight the importance of determining biotype-specific parameters in models for reliable WNV risk assessments.

\section{Acknowledgements}

We thank the Theoretical Ecology group at the University of Amsterdam for constructive comments on the modelling framework. We also thank Marcel Dicke and Samuel Brand for providing comments on an earlier version of this chapter. 



\section{Chapter 8}

Vector competence of European mosquitoes for West Nile virus

Chantal B.F. Vogels ${ }^{1 *}$, Giel P. Göertz ${ }^{2 *}$, Gorben P. Pijlman², and

Constantianus J.M. Koenraadt ${ }^{1}$

* These authors contributed equally to this work

${ }^{1}$ Laboratory of Entomology, Wageningen University \& Research

${ }^{2}$ Laboratory of Virology, Wageningen University \& Research

Submitted 


\begin{abstract}
West Nile virus (WNV) is an arthropod-borne flavivirus that circulates on the European continent between mosquito vectors and bird hosts. The main vectors for WNV in Europe are mosquito species of the Culex genus that transmit WNV among birds, and occasionally to humans, which are "dead-end" hosts. In the light of concerns about spread of the disease, several studies on European mosquito species aimed to identify which species serve as vectors for WNV, and to understand which factors influence the vector competence of these species for WNV. Here, we review the current knowledge of vector competence studies for WNV in Europe, and the molecular knowledge on physical barriers, anti-viral pathways, and microbes that can influence vector competence based on studies with other flaviviruses. By comparing the available WNV vector competence studies in Europe we evaluate the effect of factors such as temperature, mosquito origin, and mosquito biotype on vector competence. Additionally, we propose a standardized methodology to allow for comparative studies across Europe. Finally, we identify knowledge gaps regarding vector competence that, once addressed, will provide important insights into WNV transmission and ultimately contribute to effective strategies to control WNV.
\end{abstract}

Keywords: West Nile virus, vector competence, mosquito, Europe, midgut barrier, salivary gland barrier, innate immune responses, surveillance 


\section{West Nile virus in Europe}

Natural transmission cycles of the arthropod-borne West Nile virus (WNV; family: Flaviviridae) can only be established if susceptible bird hosts and competent mosquito vectors are present in a certain area under suitable environmental conditions. Mammals, including humans and equines, can occasionally become infected with WNV, but they do not develop sufficient viraemia to sustain transmission, and are therefore considered as "dead-end" hosts. Most WNV infections in humans remain asymptomatic, although an estimated one in four cases will develop into West Nile fever (reviewed in Petersen et al. 2013). Less than one percent of human WNV infections results in neurological disease such as meningitis or encephalitis, which can be fatal. In Europe, a clear distinction in cases of WNV infection in humans exists between northern and southern European countries (Figure 1). Thus far, cases of WNV infection in humans have only occurred in southern and central European countries. Due to absence of human cases in northern European countries, monitoring of WNV in these regions mainly depends on mosquito and bird surveillance programmes. Since routine surveillance programmes are not running in all European countries, and as most WNV infections in humans are asymptomatic, WNV circulation is likely to be underestimated, especially in countries without reported human or equine cases.

The differences in WNV outbreaks in humans between northern and southern Europe may be explained by two main hypotheses: 1) lower susceptibility of northern European bird populations and 2) lower vector competence of northern European mosquito species, which may be driven by colder climate. Studies from Germany and the United Kingdom have shown serological evidence of WNV circulation among resident birds (Buckley et al. 2006, Buckley et al. 2003, Linke et al. 2007). In addition, laboratory studies with carrion crows (Corvus corone; Lim et al. 2015), and European jackdaws (Corvus monedula; Lim et al. 2014), showed their susceptibility to infection with WNV. Viraemia of carrion crows (50\% tissue culture infectious dose: $>10^{5} \mathrm{TCID}_{50} / \mathrm{ml}$ ) was found to be sufficient to support WNV transmission, but this was not the case for most of the jackdaws $\left(\leq 10^{5} \mathrm{TCID}_{50} / \mathrm{ml}\right.$; Lim et al. 2015, Lim et al. 2014). The combined evidence of both field and laboratory studies on northern European birds, indicates that bird hosts are not a limiting factor for WNV transmission in northern Europe. Although corvid birds are highly susceptible to WNV infection, studies in North America have shown that other species such as the American robin (Turdus migratorius), contribute proportionally more to the WNV transmission cycle (Hamer et al. 2009, Kent et al. 2009, Kilpatrick et al. 2006). The larger contribution of American robins is mainly due to the high feeding preference of the main WNV mosquito vectors for this bird species and to the close association of robins with humans in urban areas. Thus, assessing host feeding preferences of 
European mosquito species is required to identify key bird species that can infect mosquitoes with WNV. Blackbirds (Turdus merula) and Eurasian magpies (Pica pica) are two potential key bird species which are preferred by Culex (Cx.) pipiens mosquitoes from Italy (Rizzoli et al. 2015a). Susceptibility of such key bird species to WNV needs to be determined in order to establish the role of northern European bird species in WNV transmission.

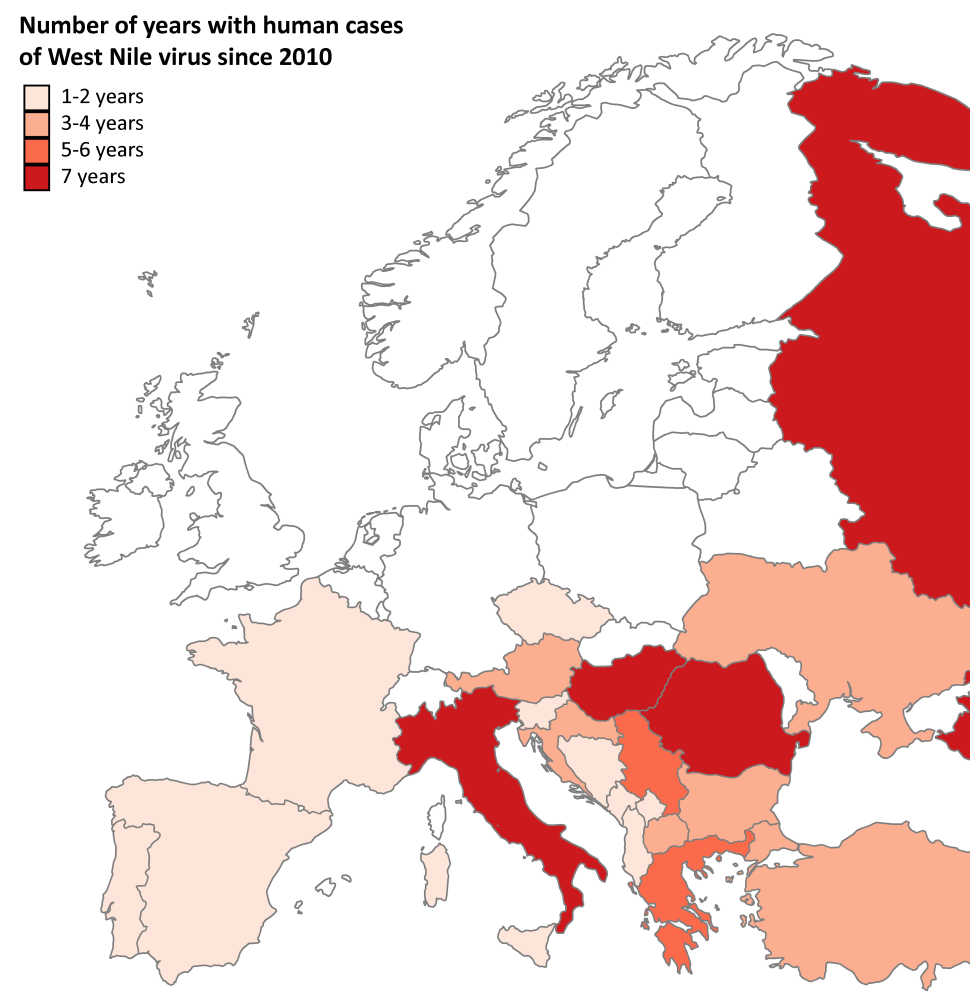

Figure 1: European countries with human cases of West Nile fever reported to the European centre for disease prevention and control (ECDC). Colour gradient indicates the number of years during which cases of WNV in humans have been reported since 2010. Reported cases are marked on the country level, except for the Balearic islands, Sicily, Sardinia, and Corsica which are individually marked. Countries with no reported cases or no data available are marked in white. Dataset provided by ECDC based on data provided by WHO and Ministries of Health from the affected countries.

Vector competence is defined as the ability of a vector to acquire, maintain, and transmit a pathogen (Kenney \& Brault 2014). In addition to vector competence, abundance and host preference are two other important traits that contribute to the efficiency with which mosquito species can transmit WNV (Kilpatrick et al. 2005). Especially lowered vector competence for WNV of northern European mosquitoes, 
due to genetic differences or environmental factors can be an alternative explanation for the differences in WNV outbreaks within Europe. Thus far, most vector competence studies on WNV have been carried out with North-American mosquito species (Goddard et al. 2002, Sardelis et al. 2001, Turell et al. 2005, Turell et al. 2001b). However, differences in species in the vector population and environment make it difficult to extrapolate results from the North-American to the European situation. Until 2014, only two studies had been published which determined the vector competence of Culex species originating from Europe for WNV (Balenghien et al. 2008, Balenghien et al. 2007). Although these studies provided insight into the possibility of WNV transmission by European mosquito species, they could not explain why WNV outbreaks seem limited to southern and central Europe. As the European Commission realized that further spread of WNV across its territories is a serious concern, several studies were launched to investigate the vector competence of European mosquito species for WNV. As a result, several new vector competence studies on European mosquito species have been published during the past three years.

This review synthesizes the results of vector competence studies on European mosquito species in order to investigate the role of intrinsic vector competence as limiting factor for WNV transmission by mosquitoes in northern Europe. The concept of vector competence is explained, including the barriers to arbovirus infection inside the mosquito body. Studies on vector competence of European mosquito species for WNV are being evaluated based on their methodology and outcomes to provide recommendations for future vector competence studies. The outcomes of the vector competence studies are linked to the available literature on the mosquito barriers, immune pathways, and interactions with other microbes, that together determine vector competence. In this way we aim for a better understanding of vector competence in European mosquitoes for WNV. Finally, recommendations for WNV surveillance in Europe and perspectives for future research are discussed.

\section{Mosquito barriers to arbovirus infection and transmission}

The outcome of the interaction between mosquito and WNV is largely dependent on the specific combination of the mosquito species and background (origin), and the WNV lineage and strain. For a successful infection of the mosquito vector, WNV has to overcome various barriers within the mosquito body: the peritrophic membrane, the midgut barrier, and the salivary gland barrier. The midgut and salivary gland barriers are both further divided into an infection and an escape barrier (Figure 2; Kenney \& Brault 2014). These barriers can limit virus infection both mechanically and through a range of antiviral immune responses, thereby determining the vector competence of the mosquito for a certain arbovirus. 
After ingestion of an infectious blood meal, virus particles travel through the foregut, cardia (proventriculus; foregut-midgut junction), and eventually end up in the midgut. Although infection of the foregut and cardia has been described for some arboviruses (Lerdthusnee et al. 1995), the majority of virus infections occur in the midgut epithelial cells (Figure 2; right inset). The first potential mosquito barrier that arboviruses encounter is the peritrophic membrane. The peritrophic membrane is not constantly present in adult mosquitoes, but forms within a few hours after uptake of a blood meal. In Culex species, formation of the peritrophic membrane can be readily observed at 2-8 hours post blood feeding, and reaches its peak thickness of $\sim 100 \mu \mathrm{m}$ after 24 hours (Houk et al. 1979).

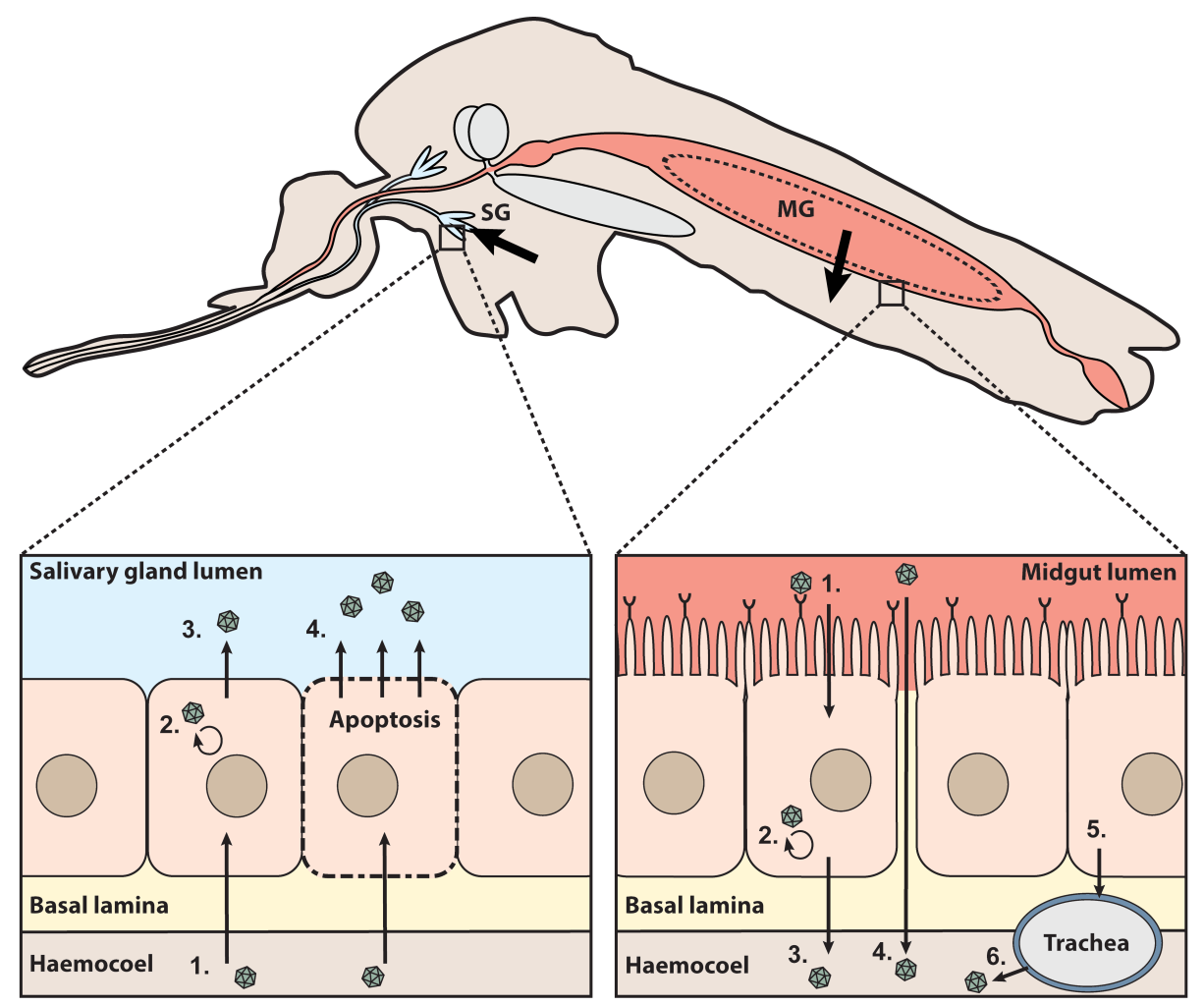

Figure 2: Schematic overview of the mosquito barriers to arbovirus infection. Schematic longitudinal cross-section of a mosquito. Arrows indicate the passage of virions through the midgut (MG) and salivary gland (SG) barriers. The dashed circle in the midgut represents the peritrophic membrane that is formed after ingestion of blood. Right inset: 1) Infection of midgut epithelial cells via binding to a putative receptor protein. 2) Virus replication in midgut epithelial cells. 3) Release of virus via budding from midgut epithelial cells and direct passage through the basal lamina into the haemocoel. 4) Direct virus passage into the haemocoel through a "leaky midgut". 5) Virus infection of trachea after budding from midgut epithelial cells. 6) Budding of virus from the trachea into the haemocoel. Left inset: 1) Infection of the salivary gland epithelial cells after passage through the basal lamina. 2) Virus replication in the salivary gland cells. 3) Virus release via budding from salivary gland cells into the salivary gland lumen. 4) Virus release from the salivary gland cells into the salivary gland lumen via apoptosis. 
The $\sim 20 \mathrm{~nm}$ pores of the peritrophic membrane (Hardy et al. 1983), are smaller than the $\sim 50 \mathrm{~nm}$ diameter of WNV virions (Girard et al. 2005). This makes it necessary for WNV to infect the midgut epithelial cells within 8 hours after ingestion of the viraemic blood meal. Indeed, flaviviruses can infect the midgut epithelial cells within 8 hours after a blood meal, and knock-down of genes required for the formation of the peritrophic membrane does not affect flavivirus infection in mosquitoes (Kato et al. 2008, Whitfield et al. 1973). Thus, despite the limited literature available, the peritrophic membrane does not seem to pose a strong barrier against WNV and other flaviviruses in mosquitoes.

Entry of flaviviruses into the midgut epithelial cells most likely occurs through interaction with cellular membrane-associated receptors (Figure 2; right inset). Flavivirus receptors in mosquitoes have not yet been identified, although some studies have suggested the involvement of cellular proteins for dengue virus (DENV) entry in C6/36 mosquito cells (Mendoza et al. 2002, Vega-Almeida et al. 2013). These proteins are required for the binding and uptake of virions during DENV infection and are either membrane-bound or membrane-associated intracellular proteins (VegaAlmeida et al. 2013). Studies with WNV in Culex mosquitoes indicated that infection in the midgut can be observed by the formation of irregular virus positive clusters of midgut epithelial cells at 3 days post initial midgut infection (dpi; McGee et al. 2010). At $5 \mathrm{dpi}$ the virus spreads more widely throughout the midgut and fully infects the midgut at $7 \mathrm{dpi}$. Studies with WNV virus-like-particles showed that initial infections occur randomly across the luminal posterior midgut epithelium, suggesting that most cells in the posterior midgut are susceptible for virus entry (Scholle et al. 2004).

After infection and replication in the midgut epithelial cells, virus particles need to disseminate through the haemocoel in order to reach the salivary glands. The haemocoel can be reached by passing through the basal lamina with or without a virus replication cycle in the midgut epithelial cells, through infection of the midgut tracheae, or through direct budding into the haemolymph. The basal lamina is a dense matrix of proteins that block large entities such as bacteria, fungi, and viruses, but is permeable to small molecules, potentially forming a barrier for flavivirus dissemination to the haemocoel (Franz et al. 2015). The thickness of the midgut basal lamina differs between different mosquito species and strains, and can affect the ability of virions to pass to the haemocoel. Differences in thickness of the basal lamina of Aedes (Ae.) albopictus did not affect the dissemination of DENV-1 (Thomas et al. 1993). Additionally, WNV particles can be observed within the intact basal lamina which suggests that WNV virions move freely within the lamina (Girard et al. 2005). These observations suggest that the basal lamina does not pose a strong barrier to flaviviruses. An alternative way of reaching the haemolymph is via infection of the tracheae. These form a network of tubular structures that reach from 
the midgut epithelial cells to the outer layer of the basal lamina. This network may allow virus passage through the basal lamina (Romoser et al. 2004). Indeed, DENV2 can infect the tracheal system and infection of the tracheae is positively correlated with virus dissemination (Salazar et al. 2007). Furthermore, it has been shown that the midgut tracheae of $C x$. pipiens can serve as a conduit for virus passage through the basal lamina, as has been shown for Rift Valley fever virus (Romoser et al. 2005). Finally, it has been shown that a small proportion of mosquitoes can have a 'leaky' midgut, resulting in direct passage of virions into the haemolymph immediately after an infectious blood meal without replication in the midgut epithelial cells (Houk et al. 1979, Weaver et al. 1991). Although leaky guts have not been shown as a characteristic of flavivirus infections, it is quite possible that in a small proportion of mosquitoes an infectious blood meal leads to direct infection of the haemolymph. So although most WNV infections will have to go through the midgut epithelial cells, some might pass this barrier by infection of the tracheae or directly through the 'leaky' midgut.

After infection of the haemolymph, virions disseminate towards and infect secondary tissues including the fat body, haemocytes, muscles, and ultimately the salivary glands. After infection of the salivary glands, viral titers increase in the saliva, and eventually reach plateau levels (Hardy et al. 1983). Female Culex mosquitoes have two salivary glands that each consist of one median and two lateral lobes (da Cunha Sais et al. 2003). The salivary gland lobes are surrounded by the salivary gland basal lamina, which forms a physical barrier to virus infection of the salivary gland epithelial cells (da Cunha Sais et al. 2003, Franz et al. 2015, Romoser et al. 2005). Although less profound than the midgut barrier, the presence of a salivary gland entry barrier has been reported for several flaviviruses in both Aedes and Culex mosquitoes (Göertz et al. 2017, Turell et al. 2006, Vazeille et al. 2013). Both the mechanical and the molecular nature of the salivary gland infection and escape barriers have not completely been defined. Most likely the basal lamina in combination with the organization of the tissue surrounding the salivary glands prevents viruses from infecting the salivary glands, while antiviral immune pathways might restrict virus replication intracellularly (Franz et al. 2015, Sim et al. 2012). Additionally, some studies have shown that the release of transmissible virus into the salivary gland lumen might require the induction of apoptosis (Clem 2016, Romoser et al. 2005).

\section{European vector competence studies on WNV}

From the $>60$ mosquito species that have been implicated as potential WNV vector species in the United States of America (USA; Hayes et al. 2005), seven species that also occur in Europe have been experimentally tested for WNV susceptibility (Table 
1). From Table 1 it becomes clear that there is large variation in the methodology of different vector competence studies. Vector competence studies have been conducted with mosquitoes collected in the field, or mosquitoes that were reared for many generations in the laboratory. Laboratory colonization can affect vector competence of mosquitoes for viruses (Gargan et al. 1983, Grimstad et al. 1977). Thus, use of field-derived mosquitoes is necessary to reliably estimate vector competence of mosquito populations in the field. Laboratory colonies which generate high numbers of mosquitoes that readily feed via artificial membrane feeders can provide a useful model system to test for underlying mechanisms of vector competence. However, one should be careful in the extrapolation of results obtained with laboratory colonies to the situation in the field.

While most studies grow WNV on C6/36 mosquito cells, others generate viral stocks on mammalian Vero cells. Generally, extensive virus passage on a specific type of cells may accumulate mutations that have an advantage for viral replication in that specific cell type, but may negatively affect the viral fitness in a vector competence experiment. For instance, WNV grown on mammalian cells loses fitness in mosquito cells (Ciota et al. 2007). Thus, WNV stocks used for vector competence studies should have a low number of passages on cells, to prevent adaptation to a specific type of cells.

Various methods of blood feeding, e.g. use of artificial membrane feeders or cotton sticks, have proven to be successful for infection of mosquitoes (Table 1). However, it remains unknown whether the choice for a particular feeding system can affect the infection success of mosquitoes (e.g. by influencing the effective dose). In addition, a large diversity of (un)natural blood sources (e.g. rabbit blood or washed erythrocytes) is being used to provide WNV to mosquitoes. Under natural conditions, transmission of WNV occurs via the bite of a mosquito on an infectious bird. Only four out of twelve vector competence studies used bird (chicken) blood to infect mosquitoes with WNV. Blood source might have consequences for the initial stages of virus infectivity in the midgut cells.

Quantification of virus is routinely done by determining the number of viral genomic RNA copies with quantitative reverse-transcription (qRT)-PCR or determining the amount of infectious virus as either the $50 \%$ tissue culture infectious dose $\left(\operatorname{TCID}_{50}\right)$ or numbers of plaque forming units (PFU). Although there is a correlation between the number of viral RNA copies and infectious virus particles, the viral genome copies are often 100-5000 times higher than the number of truly infectious virus particles (Bae et al. 2003, Choy et al. 2013). Use of infectivity assays or proper validation of qRT-PCR results is, thus, essential to draw valid conclusions on outcomes of vector competence studies. 


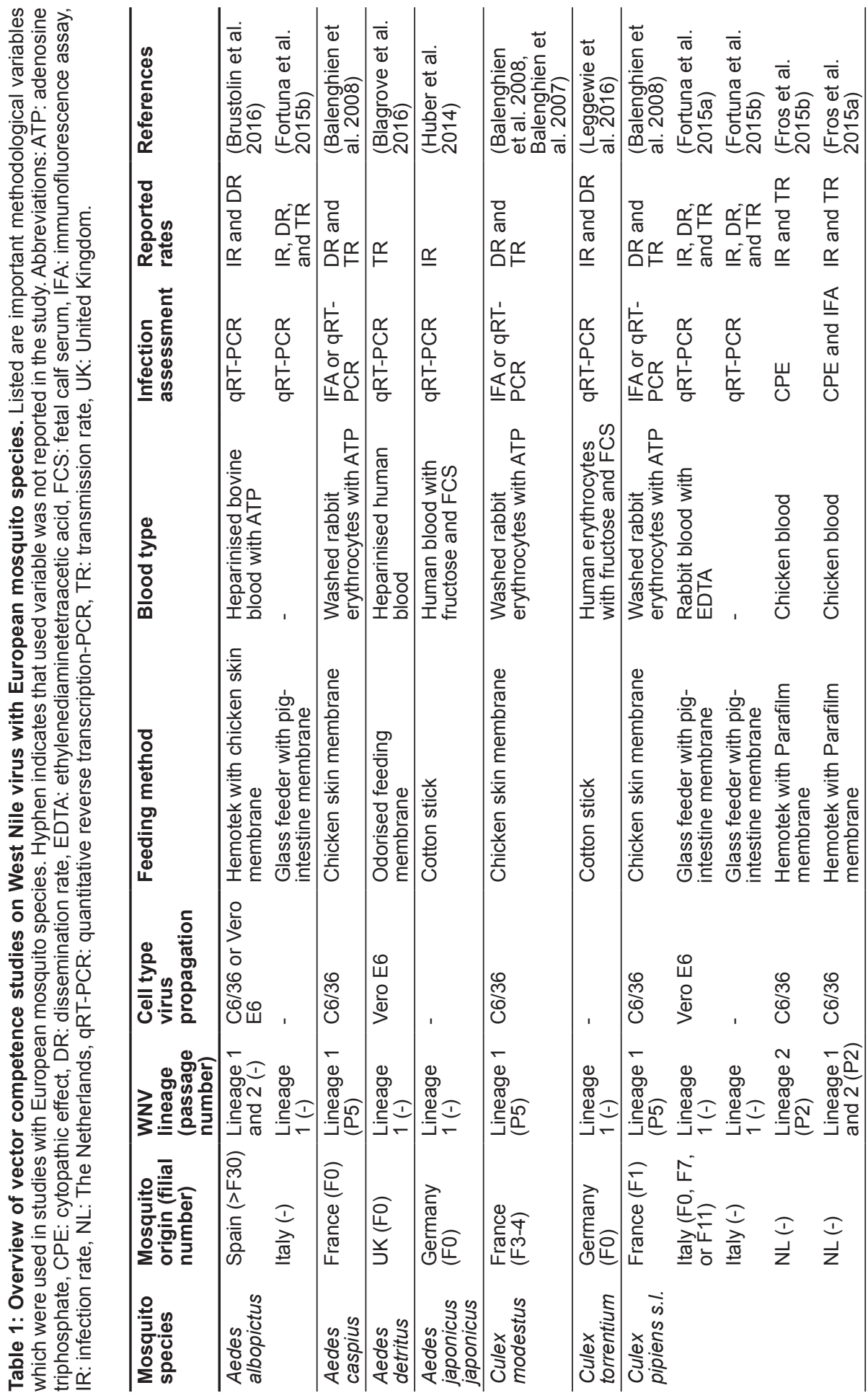




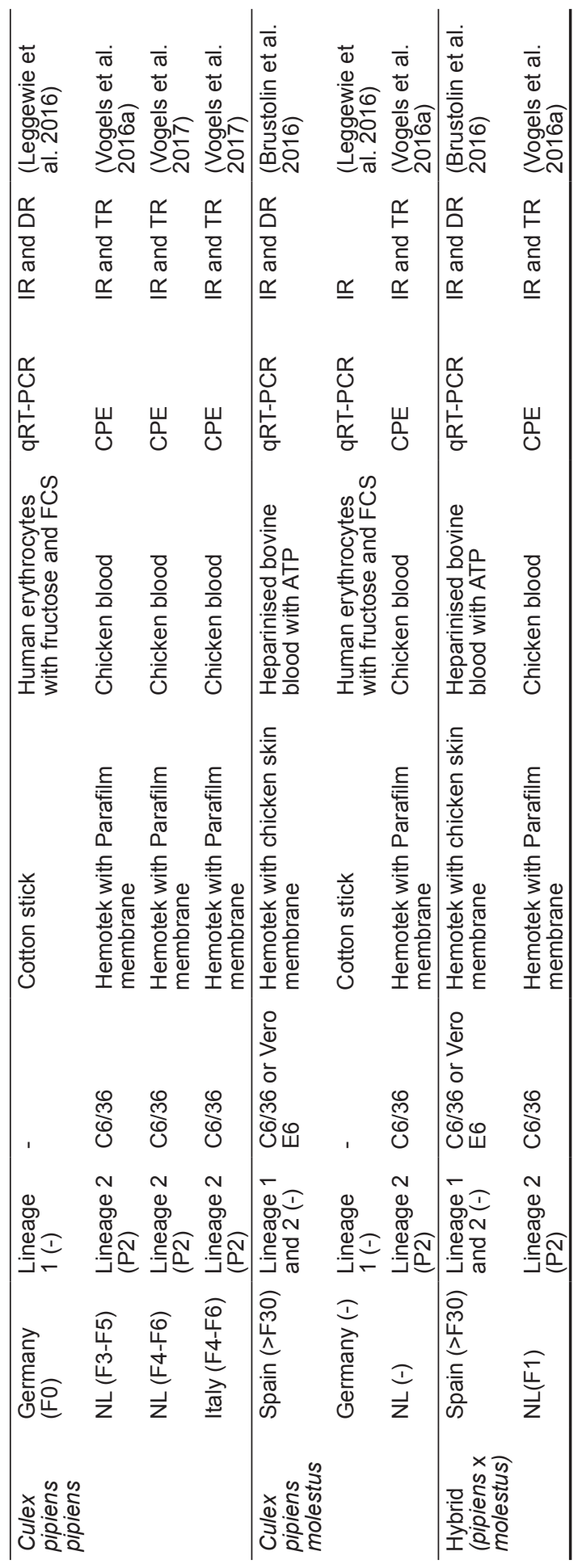


Most of the vector competence studies report a combination of infection, dissemination, and transmission rates which provide insight in the different stages of viral dissemination linked to the two main barriers inside the mosquito: the midgut and the salivary glands. Infection rates are determined based on the entire mosquito body, and represent successful infection in the midgut cells. Dissemination rates can be determined by testing peripheral mosquito body parts, such as the head or legs, and provides insight in the proportion of mosquitoes in which WNV managed to escape from the midgut cells and disseminate to the tested body part. Transmission rates are determined by testing saliva for the presence of virus, which provides insight in the proportion of mosquitoes that can actually transmit WNV. This is the most relevant parameter for vector competence. WNV transmission rates have only been determined for the following five European species: Aedes albopictus, Ae. caspius, Ae. detritus, Cx. modestus, and Cx. pipiens (Table 2). Seven out of nine studies reporting WNV transmission rates were conducted with the species $C x$. pipiens, which has been identified as one of the most important WNV vectors in the USA and Europe alike (Kilpatrick et al. 2005, Papa et al. 2013). In this review, we will mainly focus on results of those studies reporting transmission rates, because only transmission rates can provide a reliable proxy for the capability of a mosquito to transmit WNV.

Overall, WNV transmission rates of European $C x$. pipiens mosquitoes ranged between $0 \%$ and $60 \%$ (Table 2; Balenghien et al. 2008, Fortuna et al. 2015a, Fortuna et al. 2015b, Fros et al. 2015a, Fros et al. 2015b, Vogels et al. 2016a). Maximum transmission rates of northern European populations were $33 \%$ (Fros et al. 2015b, Vogels et al. 2016a), compared to up to $60 \%$ for southern European populations (Fortuna et al. 2015b). Part of this variation may be explained by intrinsic differences in vector competence of geographically distinct populations, use of different viral strains with different stock titers, or may be due to differences in study protocols (e.g. incubation time and temperature). Distinct populations of mosquitoes may differ in the effectiveness of their midgut and salivary gland barriers or impose a stronger antiviral immune response, resulting in variation in vector competence. However, in a systematic attempt to directly compare transmission rates of a northern and southern European population, thereby excluding all variation due to experimental design, no actual difference was observed in the transmission rates of both populations (Vogels et al. 2017). This suggests that there is no intrinsic difference in vector competence between northern and southern European $C x$. pipiens populations.

Temperature is another important factor which can influence vector competence. The positive effect of temperature on vector competence has been well established by previous American studies on WNV, and other arboviruses (reviewed in Kenney \& Brault 2014). Several studies confirmed that vector competence of European 
mosquitoes for WNV increases with temperature (Fros et al. 2015a, Fros et al. 2015b, Vogels et al. 2016a, Vogels et al. 2017). In the temperature range from $18{ }^{\circ} \mathrm{C}$ to 28 ${ }^{\circ} \mathrm{C}$, transmission rates of Cx. pipiens increased from $0 \%$ to $33 \%$ (Fros et al. 2015b, Vogels et al. 2016a). Thus, average northern European summer temperatures of 18 ${ }^{\circ} \mathrm{C}$ appear to be an important limiting factor for WNV transmission (Fros et al. 2015a, Fros et al. 2015b, Vogels et al. 2016a, Vogels et al. 2017).

The species $C x$. pipiens comprises two behaviourally different biotypes, pipiens and molestus, which can form hybrids. The pipiens biotype has a preference for birds as hosts (Fritz et al. 2015, Osório et al. 2014), and is thus important for the natural WNV transmission cycle. Biotype molestus and hybrids are thought to play a more important role in the spill-over of WNV from birds to humans, due to the preference of the molestus biotype for mammals, and the more opportunistic feeding behaviour of hybrids (Fritz et al. 2015, Osório et al. 2014). Thus far, two studies reported transmission rates of $C x$. pipiens mosquitoes which were identified to the biotype level (Vogels et al. 2016a, Vogels et al. 2017). One of these studies revealed that overall there is no difference in vector competence among the two biotypes in the Netherlands (Vogels et al. 2016a). However, the two biotypes did respond differentially to temperature, with increased transmission rates of biotype pipiens and hybrids, but not of biotype molestus with increasing temperatures (Vogels et al. 2016a). This shows the importance of identifying $C x$. pipiens mosquitoes to the biotype level and suggests that closely related mosquitoes may have different vector competence. Thus, the $C x$. pipiens biotypes provide the scientific community with an interesting model system for comparative studies on the underlying genetic and molecular mechanisms.

Transmission rates have also been determined for $C x$. modestus, originating from France. In general, Cx. modestus has relatively higher transmission rates of 40-55 $\%$ compared to $C x$. pipiens, and this species thus seems to be a more efficient vector of WNV (Balenghien et al. 2008, Balenghien et al. 2007).

Vector competence for WNV has only been determined for a few European Aedes species. An Italian population of the invasive mosquito species Ae. albopictus shows variation in transmission rates ranging between 0-40\%, which can be explained by different incubation periods (Fortuna et al. 2015b). An Ae. detritus population from the United Kingdom had moderate transmission rates of $21 \%$ (Blagrove et al. 2016). Dissemination and transmission rates of Ae. caspius were $1 \%$ and $0 \%$ (Balenghien et al. 2008), respectively, and infection rates of Ae. japonicus japonicus were $0 \%$ (Huber et al. 2014), which indicates that these European populations are not competent. This contrasts to the relatively high transmission rates found for $A e$. j. japonicus in the USA (Sardelis \& Turell 2001, Turell et al. 2001a). 


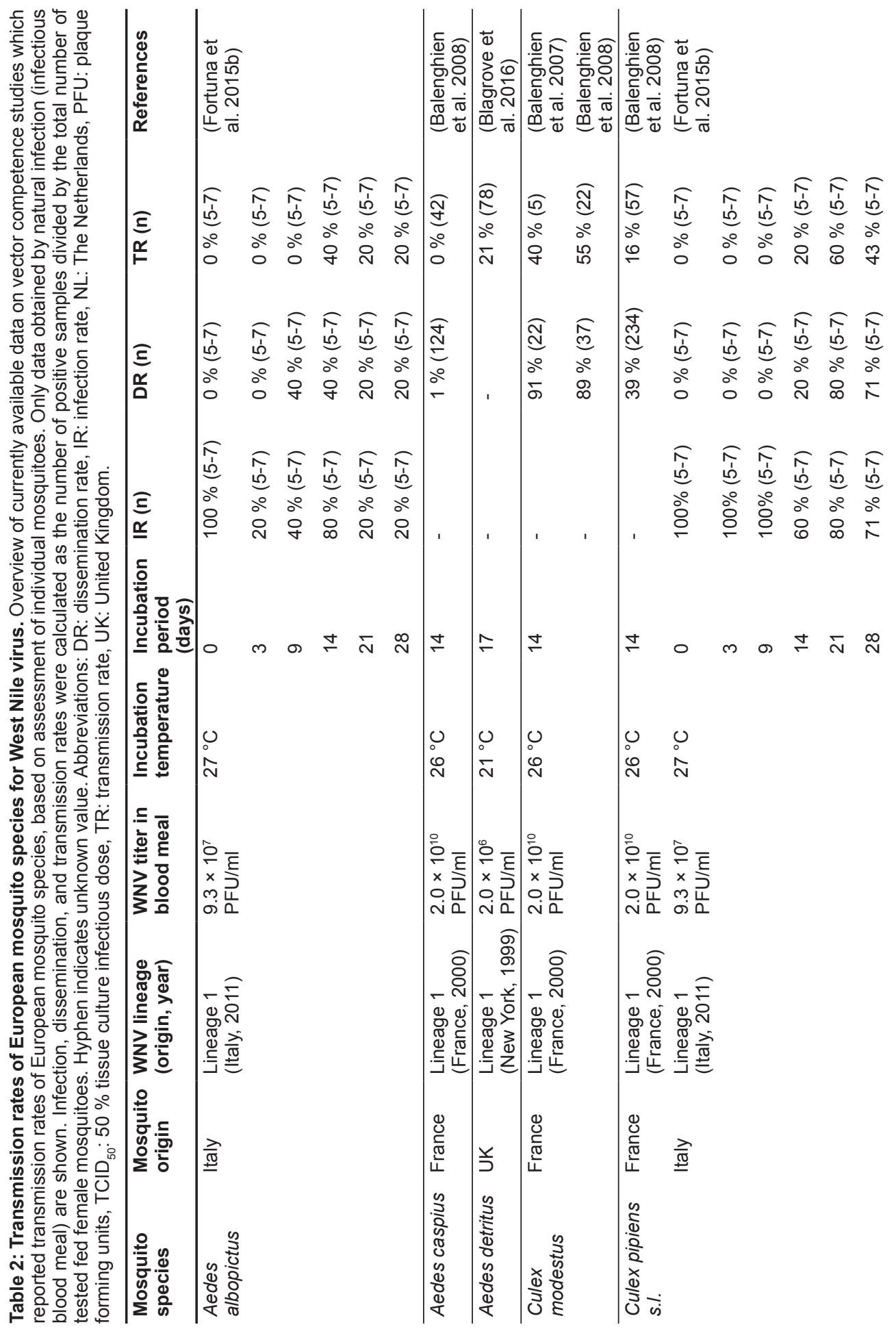




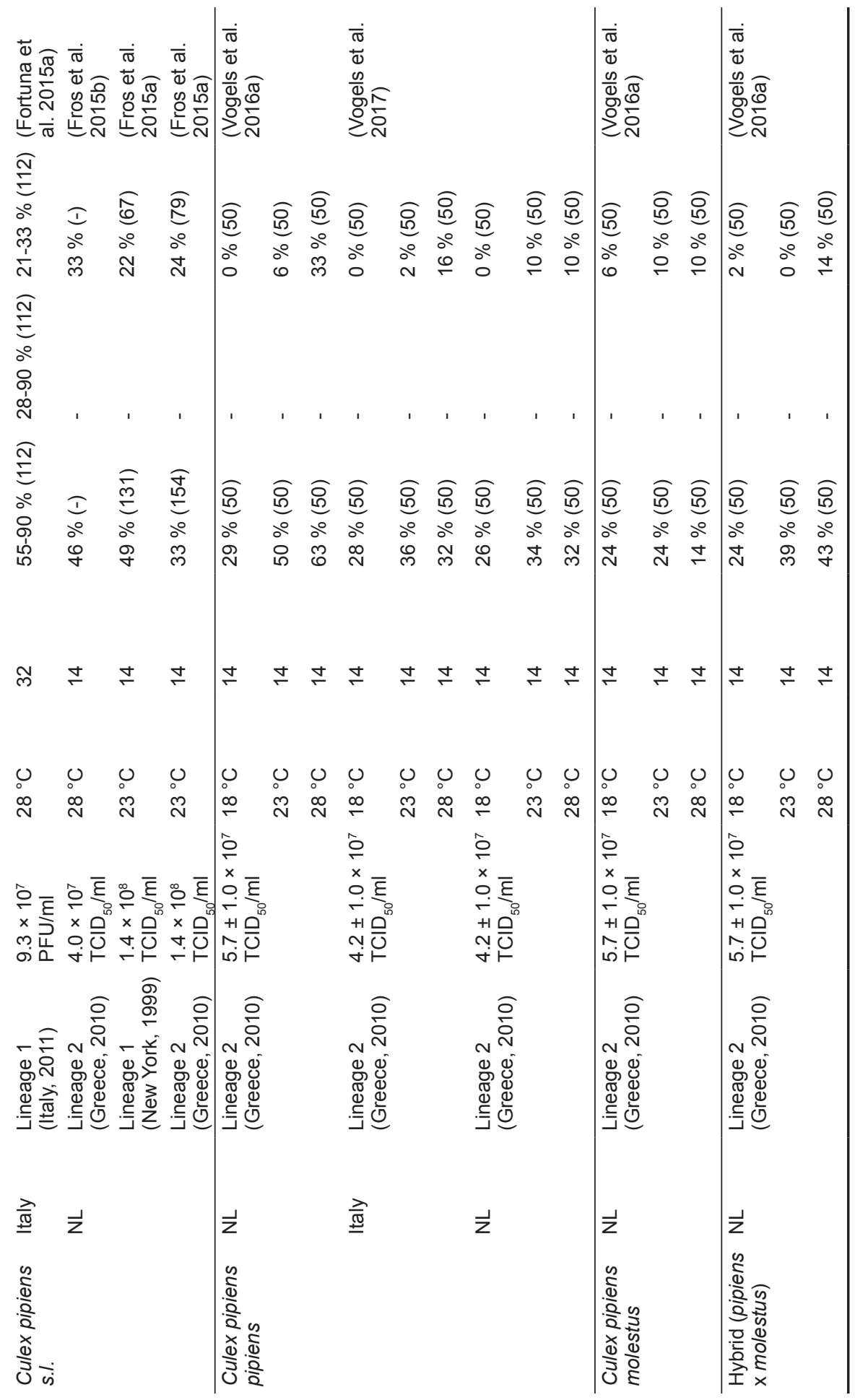




\section{Underlying mechanisms of vector competence of Culex mosquitoes for flaviviruses}

One way to study the role of the midgut barrier is to compare infection rates between mosquitoes orally exposed to WNV and mosquitoes with WNV injected into the thorax. Several studies have shown that intrathoracic injections of WNV into northern European Cx. pipiens mosquitoes results in infection rates of $\sim 100 \%$, as compared to up to $63 \%$ after an infectious blood meal where the virus has to escape from the midgut lumen by infecting midgut epithelial cells (Fros et al. 2015a, Fros et al. 2015b, Göertz et al. 2016, Vogels et al. 2016a). Thus, the lower infection rates after oral exposure indicate that $C x$. pipiens mosquitoes have a midgut infection barrier for WNV.

The same studies show that after natural infection of European Cx. pipiens via an infectious blood meal, WNV transmission rates (WNV presence in saliva) are lower compared to WNV infection rates (WNV presence in body). This suggests a role of either the midgut escape barrier or the salivary gland barrier. The presence of a salivary gland barrier can be assessed by comparing transmission rates between orally exposed and injected mosquitoes. If no salivary gland barrier exists, then it is expected that mosquitoes which are injected with WNV in the thorax have transmission rates of approximately $100 \%$. Indeed, it has been repeatedly shown that intrathoracic injections result in transmission rates of $\sim 100 \%$ in Cx. pipiens mosquitoes, indicating that there is no important role for the salivary gland barrier in WNV transmission (Fros et al. 2015a, Fros et al. 2015b, Göertz et al. 2016, Vogels et al. 2016a). However, studies on Cx. modestus do suggest a role for the salivary gland barrier, as they show lowered transmission rates of $40-50 \%$ compared to dissemination rates of 89-91 \% (Balenghien et al. 2008, Balenghien et al. 2007). The differences between transmission rates after oral infection and intrathoracic injection studies are likely the result of a midgut escape barrier, artificially high WNV titers injected at once close to the salivary glands, or absence of selective pressure of the midgut barrier bottle neck on the virus population (Brackney et al. 2009).

As demonstrated above, the vector competence of European mosquitoes is mainly dependent on the ability of the virus to overcome the midgut and salivary gland barriers. It is, therefore, interesting to study the underlying mechanism of these barriers in order to further understand the basis for the vector competence of European mosquitoes for WNV.

\section{Innate immune responses}

As a defence to incoming pathogens, mosquitoes have evolved several antiviral response pathways, such as the RNA interference (RNAi), Janus Kinase/Signal 
Transducer and Activator of Transcription (JAK/STAT), Toll, immune deficiency (IMD) and apoptosis pathways (Prasad et al. 2013). Additionally, experimental evidence suggests that heat shock proteins and reactive oxygen species also act antiviral, but due to the limited knowledge available on these responses they will not be discussed here (reviewed in Saraiva et al. 2016). The expression of genes that encode proteins and factors that function in the mosquito innate immune pathways can be upregulated or activated after ingestion of an infectious blood meal. In this way, these immune pathways play an important role in the midgut and possibly the salivary gland barriers that in turn contribute to the vector competence of a mosquito for certain arboviruses. Different mosquito species might have variation in the effectiveness of their antiviral immune responses against certain arboviruses. Such variation might explain differences in vector competence of different mosquito species for certain viral strains. Most knowledge on the invertebrate immune responses is derived from research on Drosophila, although there is an increasing numbers of studies on immunity against alphaviruses and flaviviruses, mostly conducted on Aedes mosquitoes. Here we review the literature concerning flavivirus infections and their role in triggering the mosquito antiviral immune responses in both Aedes and Culex species mosquitoes.

RNAi is one of the most important immune responses of invertebrate vectors (Blair 2011). The RNAi response is an antiviral response of insects that is induced upon recognition of non-self double-stranded (ds)RNA. Even though flaviviruses are single stranded (ss)RNA viruses, dsRNA intermediates are produced during viral RNA replication, that serve as substrates for the endoribonuclease Dicer-2 to activate the RNAi response. Additionally, the genomic RNA can fold into dsRNA secondary structures that can be recognized by the RNAi machinery of the mosquito. Activation of the RNAi response leads to degradation of the viral genomic RNA through cleavage by Argonaute-2, resulting in decreased virus replication. Various studies have shown that viral small interfering RNAs are produced during infection of Usutu, DENV-2 and WNV in mosquitoes (Fros et al. 2015b, Göertz et al. 2016, Hess et al. 2011). As these small interfering RNAs are the result of an active antiviral RNAi response, this indicates that the RNAi response is initiated upon infection of arboviruses in mosquitoes, and might influence the susceptibility of these mosquitoes for arbovirus infection. RNAi is capable of controlling DENV infection in Ae. aegypti mosquitoes and results in decreased viral titers and transmission rates (Franz et al. 2006). Additionally, silencing of important effector proteins in the RNAi pathway increases DENV-2 infection rates and titers in Ae. aegypti (Sánchez-Vargas et al. 2009). Interestingly, in Cx. quinquefasciatus RNAi targets the WNV genomic RNA in the mosquito midguts (Brackney et al. 2009). Correspondingly, a mutant WNV that lacks a potential viral suppressor of RNAi (based on in vitro studies) has decreased 
transmission rates after an infectious blood meal, but not after intra-thoracic injection (Göertz et al. 2016). This observation suggests that WNV requires this potential RNAi suppressor to suppress the RNAi response in the mosquito midgut and thereby overcome the midgut barrier.

The invertebrate JAK/STAT pathway is the insect innate immune pathway that resembles the mammalian interferon response (Fragkoudis et al. 2009). The JAK/ STAT pathway is initiated upon virus recognition by a transmembrane receptor that activates a signaling cascade which upregulates the expression of antiviral response genes. These antiviral genes act by recognizing virus infection, suppressing virus replication, and by triggering nearby cells to enter an antiviral state. Although strongly established as an antiviral defense system in Drosophila (reviewed in Arbouzova \& Zeidler 2006), the influence of the JAK/STAT pathway on flavivirus infection in mosquitoes remains to be resolved. One study with DENV-2 in Ae. aegypti mosquitoes showed that silencing either components or effectors of the JAKSTAT pathway increases DENV-2 titers, while silencing of a JAK/STAT antagonist decreases DENV-2 titers (Souza-Neto et al. 2009). This suggests that under natural conditions, the Ae. aegypti JAK/STAT pathway restricts DENV-2 and influences the vector competence. In Ae. aegypti, infection with WNV and DENV has been shown to modulate the expression of JAK/STAT-related-genes (Colpitts et al. 2011, Xi et al. 2008). WNV infection in Cx. quinquefasciatus activates the JAK/STAT transcription factor STAT1A, suggesting that the JAK/STAT pathway is involved in controlling WNV infection. However, this activation has not been shown to restrict WNV dissemination or transmission (Bartholomay et al. 2010). In Cx. quinquefasciatus, activation of the RNAi pathway increases expression of the cytokine Vago, that is secreted from infected cells, to subsequently activate the JAK-STAT signaling cascade in neighboring cells. Through this indirect mechanism, Vago thus decreases WNV titers in a JAK/STAT dependent manner (Paradkar et al. 2012), supporting the role of the JAK/STAT pathway in controlling flavivirus infections in Culex mosquitoes.

Another conserved immune response in mosquitoes is the Toll pathway. The Toll pathway is activated after recognition of virus infection by Toll-like-receptors. This eventually results in the expression of antiviral effector genes (reviewed in Sim et al. 2014). DENV-2 infection of Ae. aegypti mosquitoes activates the main components of the Toll pathway by increasing their transcriptional level (Xi et al. 2008). Depletion of Toll regulators results in increased DENV-2 and DENV-4 titers, whereas silencing of a Toll pathway inhibitor results in decreased DENV titers (Ramirez \& Dimopoulos 2010, Xi et al. 2008). DENV infection of salivary glands in Ae. aegypti results in upregulation of Toll pathway related genes in the salivary glands, indicating that the salivary glands can mount an antiviral response against flaviviruses (Sim et al. 2012). Transcriptomic studies have shown that components of the Toll pathway are 
also differentially expressed during Yellow Fever virus infection, but not during WNV infection (Colpitts et al. 2011). In another study, infection of Cx. quinquefasciatus with WNV led to altered regulation of various genes, but Späetzle-like genes are the only Toll-related genes that were modulated (Bartholomay et al. 2010).

The IMD pathway is activated upon recognition of virus infection by the adaptor IMD protein, subsequently resulting in the transcription of IMD effector genes (reviewed in Sim et al. 2014). Studies with DENV-2 have shown that infected Ae. aegypti have increased expression of IMD pathway components (Luplertlop et al. 2011, Xi et al. 2008). However, silencing of the IMD antagonist Caspar does not affect DENV-2 titers, suggesting that the IMD pathway is not involved in controlling DENV-2 infection in Ae. aegypti (Xi et al. 2008). Interestingly, silencing of the IMD pathway in a DENV refractory Ae. aegypti population resulted in increased DENV-2 replication suggesting that the IMD pathway contributes to the refractoriness of this mosquito population (Sim et al. 2013). WNV infection in Cx. quinquefasciatus does not affect the regulation of IMD related genes (Bartholomay et al. 2010), although more studies are required to understand the role of the IMD pathway during WNV infections in Culex.

Apoptosis (programmed cell death) is considered an antiviral response by the host to eliminate viral infection by killing infected and neighboring cells. Many viruses inhibit apoptosis in order to facilitate their replication and to prevent clearance of the infected cells. Interestingly, some arboviruses utilize cellular caspases during replication or use apoptosis as a measure to release virions from infected cells. WNV infection in $C x$. pipiens causes apoptosis in the midgut cells, mainly in the anterior region (Vaidyanathan \& Scott 2006). Infection of Ae. aegypti mosquitoes with DENV results in upregulation of apoptosis-related-genes. For some genes that function in the apoptosis pathway, silencing resulted in increased DENV replication (Barón et al. 2010). Additionally, DENV infection of both a refractory and susceptible strain of Ae. aegypti induced expression of the apoptotic gene $m x$ as soon as 3 hours post blood meal in the refractory, but not in the susceptible strain (Liu et al. 2013). These observations suggest that apoptosis acts as an antiviral response in the midgut. Also in the salivary glands, WNV infection causes apoptotic-like cell death, most likely due to the triggering of cellular apoptotic pathways as a result of active virus replication (Girard et al. 2005, Girard et al. 2007). Additionally, infection of Cx. quinquefasciatus salivary glands with WNV resulted in a decrease in a Culex apoptosis inhibitor (Girard et al. 2010). Although apoptosis is, thus, induced in the salivary glands, virus titers do not rapidly decline over time, suggesting that apoptosis is not effectively clearing the virus infected cells in the salivary glands (Girard et al. 2007). Alternatively, virusinduced apoptosis in the salivary glands might increase the release of transmissible virus into the saliva thereby actually facilitating virus transmission. 


\section{The mosquito microbiome}

Mosquitoes live in symbiosis with a wide variety of bacteria and fungi, which are abundantly present in the mosquito gut and are referred to as the mosquito gut microbiome (Hegde et al. 2015). These microorganisms interact with the mosquito gut and have a strong impact on the mosquito metabolism, but also affect interactions with pathogens (Jupatanakul et al. 2014). Additionally, mosquitoes can be persistently infected with insect-specific viruses that normally do not affect the mosquito life cycle, but can have an effect on the ability of the mosquito to become infected with other viruses (Salas-Benito \& De Nova-Ocampo 2015). The microbiome of Ae. aegypti is important for successful infection of flaviviruses, as has been shown for DENV-2 (Hegde et al. 2015). The introduction of specific bacterial strains into the mosquito gut microbiome can affect the infection or replication of flaviviruses in mosquitoes either in a positive or negative way, which in turn may affect their vector competence for certain viruses (Hegde et al. 2015).

Apart from bacteria in the mosquito midgut, some bacteria are present inside the cells of mosquitoes and are transmitted both horizontally between species and vertically to the mosquito offspring (O'Neill et al. 1997). The best documented intracellular bacterium of mosquitoes is Wolbachia. Wolbachia is an endosymbiotic bacteria that can affect vector competence for arboviruses (reviewed in Johnson 2015). Although there is a large body of literature describing the interactions between flaviviruses and Wolbachia, we will here discuss those studies addressing the effect of Wolbachia on transmission and replication of WNV only. Wolbachia infection increased WNV replication, but resulted in less infectious virus production, suggesting that Wolbachia interferes with virion assembly within or secretion of virus from mosquito cells (Hussain et al. 2013). Consequently, Ae. aegypti mosquitoes infected with Wolbachia have lower WNV infection and transmission rates. Infection of $C x$. quinquefasciatus with Wolbachia results in increased resistance to WNV infection via a blood meal and lower viral titers in the mosquito body as compared to mosquitoes cleared from Wolbachia with tetracycline (Glaser \& Meola 2010). However, Cx. tarsalis mosquitoes infected with Wolbachia showed enhanced WNV infection rates due to down regulation of Toll-pathway related genes by Wolbachia (Dodson et al. 2014). Accordingly, a study on Wolbachia in field-caught Cx. quinquefasciatus populations showed that densities (i.e. the bacterial load) of Wolbachia were too low to inhibit WNV infection and did not affect vector competence of these same mosquito colonies. Thus, for $C x$. quinquefasciatus the virus inhibitory effect depends on the density of Wolbachia in the mosquito (Micieli \& Glaser 2014). The effect of Wolbachia on vector competence of mosquitoes is further dependent on the strain of Wolbachia in combination with the species of mosquito used (Johnson 2015). Taken together infection with Wolbachia can have a strong effect on vector competence of 
mosquitoes for WNV, although the mechanism remains unclear.

\section{Discussion}

We have reviewed the available literature on vector competence of European mosquito species for WNV, and we present an overview of knowledge on the underlying mechanisms to understand the basis of vector competence for WNV. Four European mosquito species, Ae. albopictus (Fortuna et al. 2015b), Ae. detritus (Blagrove et al. 2016), Cx. modestus (Balenghien et al. 2008, Balenghien et al. 2007), and Cx. pipiens (Fortuna et al. 2015a, Fortuna et al. 2015b, Fros et al. 2015a, Fros et al. 2015b, Vogels et al. 2016a, Vogels et al. 2017), have thus far been confirmed to be able to transmit WNV in the laboratory. Two Aedes species, Ae. caspius and Ae. japonicus japonicus, were not competent as vector for WNV (Balenghien et al. 2008, Huber et al. 2014). Highest transmission rates were found for $C x$. pipiens, which in combination with their relatively high WNV infection rates in the field (Engler et al. 2013), and their high abundance during summer (Chaskopoulou et al. 2016), highlights the importance of European $C x$. pipiens mosquitoes in the transmission of WNV.

Importantly, no intrinsic differences in vector competence were found in a direct comparison between a northern and southern European $C x$. pipiens population, with increased transmission for both populations at higher temperatures (Vogels et al. 2017). This confirms that temperature is likely the most important factor to explain why WNV outbreaks have thus far been limited to southern and central Europe (Fros et al. 2015a). However, considering the presence of intrinsically competent mosquito vectors (Balenghien et al. 2008, Balenghien et al. 2007, Blagrove et al. 2016, Fortuna et al. 2015a, Fortuna et al. 2015b, Fros et al. 2015a, Fros et al. 2015b), and susceptible bird hosts (Lim et al. 2015, Lim et al. 2014), together with predictions of more frequent and prolonged temperature anomalies, we see no limiting factors that can rule out future WNV circulation in northern Europe. In fact, the recent circulation of Usutu virus in northern Europe during the autumn of 2016 (Cadar et al. 2017), exemplifies that there are no evident restrictions for Culex-borne flaviviruses to become endemic in northern Europe. Because of the similar transmission cycle and comparable transmission rates of both Usutu and West Nile virus with their main vector Cx. pipiens (Fros et al. 2015b), we consider the circulation of Usutu virus as a prelude to WNV transmission (Fros et al. 2015b).

\section{Implications for WNV surveillance}

Several European countries such as Italy and Greece, have implemented WNV surveillance programmes by monitoring mosquitoes, (sentinel-)birds, (sentinel-) 
equines, or humans for presence of virus, seroconversion, or disease symptoms, in order to gain insight in the dynamics of WNV circulation (reviewed in Engler et al. 2013). With the knowledge presented in this review, we recommend to implement WNV surveillance programmes throughout Europe. We favour integrating national surveillance initiatives into a Europe-wide surveillance network, as suggested by Engler et al. 2013. This should result in the use of general protocols which allows for comparisons across Europe. However, selection of appropriate surveillance tools should still be based on the specific needs of individual countries.

Such surveillance programmes can then either be active through the screening for virus in living organisms or passive, involving the screening of dead birds, or based on serology (Engler et al. 2013). Active screening of mosquitoes or sentinel-birds allows for detection early in the season, whereas passive screening of dead birds or neurological disease cases is most useful to provide insight in emergence of WNV into new areas. Detection early in the season allows for awareness, targeted mosquito control, and personal protection which can reduce WNV outbreaks in humans later in the season. This form of surveillance is, therefore, highly recommended for countries which encounter yearly cases of WNV in humans, but is to a lesser degree essential for countries in which no human cases have yet emerged. Active mosquito surveillance is costly and WNV is usually only detected in a few percent of mosquito pools (Engler et al. 2013), even during outbreaks, thereby making passive surveillance of suspected cases more suitable for countries with currently no known cases of WNV in humans.

\section{Recommendations for vector competence studies}

Considering the vector competence studies of European mosquito species for WNV, it becomes clear that the large variation in study protocols makes it difficult to directly compare studies. Variation in techniques, and choice of mosquito populations and viral strains, all have an effect on the outcomes of vector competence studies. Therefore, to enable comparison between vector competence studies, detailed information on the mosquito vector, virus strain, and technical details need to be reported. Such information should at the minimum include accurate identification of the mosquito vector, exact origin of mosquito populations (coordinates), mosquito generation, mosquito rearing protocols, incubation temperature, and incubation time. Information regarding the virus isolate should include the viral strain used (including GenBank reference), virus passage number, cell types used to grow virus stocks, and technical details on how the virus stock was generated. Technical details should include detailed descriptions of blood feeding methods (including the blood source) and infectivity assays. In addition, future research consortia should discuss and adjust research protocols in order to increase comparability between studies 
from different laboratories. Inclusion of more detailed information and consistency in research protocols allows for better comparison between studies and makes it easier to draw conclusions on observed results.

Choice of incubation time and temperature can strongly influence the outcome of vector competence studies. Studies that focused on the effect of incubation time (Fortuna et al. 2015b), and incubation temperature (Fros et al. 2015a, Fros et al. 2015b, Vogels et al. 2016a, Vogels et al. 2017), confirmed their effects on transmission rates. For studies aiming to determine vector competence of a certain mosquito species, we recommend to use fixed incubation periods and temperatures in order to increase comparability between studies. Based on previous studies, we suggest to at least include incubation periods of 14 days, and incubation temperatures that represent average summer temperatures of the region the mosquito population originates from, as well as a standardised temperature of $27 \pm 1{ }^{\circ} \mathrm{C}$. Standardizing vector competence protocols will largely increase the ability to make comparisons between studies, which is essential to increase the understanding on WNV transmission in Europe.

Vector competence studies can provide information such as infection, dissemination and transmission rates, which provide insights in the likelihood of the vector to transmit the virus in the field. Some studies also include the rate at which a model organism can become infected by a viraemic mosquito, which gives a better proxy for transmission in the field. However, since these studies are labour intensive, and can often only be performed with a small number of individuals due to ethical constraints and costs, assessing the presence of virus in the saliva is, to our opinion, the best alternative to estimate transmission rates.

Several European vector competence studies only tested infection or dissemination rates based on mosquito bodies, legs, or head squashes. Although this provides insight in the potential of species to become infected with WNV, it does not provide conclusive evidence for a role of these mosquitoes in WNV transmission. Additionally, reporting infection, dissemination, and transmission rates increases the understanding as to where the bottlenecks for transmission of a certain virus are. Therefore, we strongly recommend that future vector competence studies determine transmission rates by measuring virus in the saliva in order to better understand the state of WNV transmission in Europe.

\section{Directions for future research}

The vector competence studies with European mosquitoes have shown that some European mosquitoes are competent to transmit WNV, whereas other mosquito species appear to be less or not competent at all. Although this indicates that 
surveillance for WNV in European mosquitoes is necessary, it does not answer the question why some European vectors are more competent than others. In order to fully understand arbovirus transmission dynamics, it is important to identify the determining factors of vector competence. Especially the influence of the mosquito barriers, immune responses (specifically against flaviviruses), and microbes on vector competence are still poorly understood. Studying the underlying mechanisms of how these barriers and responses influence vector competence in mosquitoes is therefore important to understand variation in vector competence.

Understanding the molecular mechanisms of virus transmission allows for more directed control approaches by e.g. targeting barrier specific genes or application of microorganisms that reduce vector competence. Various vector control strategies have been proposed based on our current understanding of the mosquito's immune system such as 1) the development of transmission blocking vaccines, 2 ) the generation of refractory mosquitoes through genetic modification of either the mosquito or 3) the mosquito's microbiome and 4) infection of mosquitoes with intracellular bacteria such as Wolbachia (Gonçalves \& Hunziker 2016, Johnson 2015, Wang \& Jacobs-Lorena 2013). Although no attempts have been made to employ the mosquito's immune system for the control of arboviral disease, promising results have been made in field studies with Wolbachia-infected mosquitoes. Studies with Wolbachia-infected Aedes mosquitoes in Australia and Vietnam have already shown the potential of Wolbachia to invade the local mosquito population (Nguyen et al. 2015). While the effect on virus-transmission in the field is yet to be determined, this study indicates the feasibility of releasing Wolbachia-infected mosquitoes as an arboviral control strategy. Potentially, similar release strategies could be performed with genetically modified mosquitoes that employ the mosquito's immune system to further prevent the transmission of arboviruses.

As the most important vector species for WNV transmission in Europe is Cx. pipiens, more research on virus-vector interactions in $C x$. pipiens is required to overcome the challenges related to virus control. Fortunately, the genome sequence of $C x$. quinquefasciatus, the first sequenced genome of a Culex species, is available and allows for genetic studies with Culex mosquitoes. However, as the genome sequence of $C x$. pipiens is not available, genetic studies should be performed with $C x$. quinquefasciatus mosquitoes. Alternatively, attempts can be made to perform de novo transcriptomics in $C x$. pipiens and compare the outcomes with the annotation of the $C x$. quinquefasciatus genome, although such an approach is likely to miss some candidate genes due to genetic variations.

Thus far, most studies on mosquito immune pathways involved in flavivirus infections have been done with Aedes mosquitoes. The limited research on mosquito immune 
pathways in Culex has confirmed the presence of the Toll, IMD, JAK/STAT and RNAi pathway, as putative antiviral responses towards flavivirus infections. Yet, the relative contribution and effectiveness of these pathways in controlling transmission of WNV and other flaviviruses by Culex mosquitoes is still unclear and requires further investigation. Potentially, such studies can indicate novel antiviral responses that are unique for Culex species such as the antiviral Vago response that has thus far only been observed in Culex mosquitoes (Paradkar et al. 2012). Studies that aim to understand the molecular interactions between the vector and the virus often rely on controlled experiments in cell lines. Culex cell lines are available for the species $C x$. quinquefasciatus (Hsu) and Cx. tarsalis (CxT), but a cell line of $C x$. pipiens is not available. This limits studies on immune responses and virus replication in $C x$. pipiens mosquitoes to extrapolations from cell lines of other Culex species. Because $C x$. pipiens is the predominant vector species for WNV in Europe, a Cx. pipiens cell line would greatly contribute to research that aims to understand the basis of vector competence for WNV in European mosquitoes.

Studies that investigated the effect of temperature on the transmission of WNV by European mosquitoes have shown that increasing temperatures result in higher transmission rates (Table 2). Indeed, higher temperatures can lead to higher virus replication rates. Alternatively, temperature might have an effect on immune regulatory pathways or the microbiome, resulting in altered vector competence (Murdock et al. 2012). Interestingly, it has been shown that at low temperatures of $18{ }^{\circ} \mathrm{C}$, the RNAi pathway is less effective in Ae. aegypti resulting in increased susceptibility to arbovirus infection (Adelman et al. 2013). Similar studies in Culex mosquitoes could highlight why the vector competence changes with alternating temperature or whether it is simply the lowered virus replication at lower temperatures.

\section{Conclusions}

Several mosquito species from both northern and southern Europe are competent vectors for WNV. Considering the various vector competence studies done across a range of temperature regimes, we see no clear restriction for future WNV circulation at more northern latitudes, especially in the light of climate change. We recommend WNV surveillance throughout Europe, with specific programmes adjusted to the needs of individual countries, by taking the current status of WNV circulation into account. Protocols for future vector competence studies on WNV should be streamlined in order to allow for better comparison between studies on the European scale. Future research should, however, not only focus on investigating vector competence of additional mosquito species, but it should rather be directed at understanding underlying mechanisms of vector competence for WNV. We foresee 
great potential in research directions focused on the effects of the mosquito immune response and microbiome on vector competence.

\section{Acknowledgements}

We thank Marcel Dicke and Monique van Oers for feedback on an earlier version of this chapter. 



\section{Chapter 9}

General discussion: Identification of mosquito-related factors that limit West Nile virus transmission in northern Europe 


\section{Introduction}

Mosquitoes play a key role in the transmission of arthropod-borne (arbo)viruses of medical and veterinary importance. One of the most widespread arboviruses in the world is West Nile virus (WNV; family: Flaviviridae; Chancey et al. 2015). Worldwide outbreaks of WNV are recurring, and lead to disease in thousands of humans and equines every year (CDC 2015, ECDC 2016). Despite the worldwide emergence, WNV outbreaks are not uniformly spread across all continents. Europe is a continent of particular interest, because there seems a clear dichotomy between northern countries which have never reported WNV infections in humans, and southern and central countries which experience WNV infections in humans on a yearly basis (Calistri et al. 2010a, ECDC 2016). Especially the northern European countries without WNV outbreaks are interesting to study, in order to enhance our understanding of factors that limit WNV transmission.

The success of the widespread emergence of WNV can be found in the ecology of the WNV transmission cycle. Arboviruses are transmitted between vertebrate hosts by arthropod vectors. Unlike most arboviruses that restrict their replication to a narrow range of vectors and hosts, WNV has a remarkably broad vector- and hostrange involving multiple mosquito vector and bird host species (Hayes et al. 2005). Related flaviviruses, such as dengue and Zika virus, are predominantly maintained in urban transmission cycles between two main mosquito vectors, Aedes aegypti and Aedes albopictus, and human hosts (Chouin-Carneiro et al. 2016, Weaver \& Barrett 2004). In contrast, WNV has been detected in more than 60 mosquito and 300 bird species in the United States (USA) alone (CDC 2012a, CDC 2012b, Komar 2003). Involvement of such a high number of vectors and hosts increases the complexity of the ecology of WNV. However, despite the large number of potential vectors for WNV, only few species have been identified as main contributors to transmission (Kilpatrick et al. 2005, Komar 2003, Turell et al. 2005). One of these main contributors is the northern house mosquito, Culex (Cx.) pipiens (Fonseca et al. 2004, Kilpatrick et al. 2005). Until a few years ago, most studies on WNV transmission were executed with American Cx. pipiens populations (Ciota et al. 2013a, Dohm et al. 2002, Kilpatrick et al. 2010, Kilpatrick et al. 2008). However, to understand the role of $C x$. pipiens in transmission of WNV in Europe, studies on European mosquito populations were warranted.

Differences in the ability to transmit WNV (i.e. vector competence) between northern and southern European $C x$. pipiens populations may possibly explain the absence of WNV outbreaks in northern parts of Europe. The aim of this thesis was to investigate the role of $C x$. pipiens mosquitoes in transmission of WNV in Europe, in order to understand differences in WNV circulation between northern and southern Europe. 
Central in my work are the spatial distribution and population composition of $C x$. pipiens mosquitoes, and their vector competence for WNV in relation to temperature. In this chapter, I will discuss the implications of my findings and evaluate the risks of WNV transmission and establishment for northern Europe.

\section{Mosquito-related determinants for West Nile virus establishment}

The likelihood of disease establishment can be predicted with basic reproduction number $\left(R_{0}\right)$ models (Anderson \& May 1990, Diekmann \& Heesterbeek 2000, Macdonald 1952). The $R_{0}$ is a measure for the number of secondary cases that can arise after introduction of one infectious individual in a susceptible population. Outcomes of $R_{0}$ models provide a prediction for the likelihood of disease establishment, which is only likely to occur if $R_{0}$ is larger than one. The $R_{0}$ is dependent on interactions between vectors (e.g. vector competence, extrinsic incubation period, survival, and biting rate) and hosts (e.g. host susceptibility, and duration of infectious period), which can be influenced by the environment (e.g. temperature). Each of the factors included in these $R_{0}$ models may explain differences in WNV transmission across Europe (chapter 7 ). In the following sections, all mosquito-related factors that underlie the $R_{0}$ are being discussed in order to identify factors that can explain the absence of WNV outbreaks in northern Europe.

\section{Culex pipiens population composition}

The species $C x$. pipiens consists of two behaviourally different biotypes, named pipiens and molestus (Byrne \& Nichols 1999). With regard to WNV transmission, one of the most important behavioural differences between the two biotypes is their host preference. Biotype pipiens has a preference for birds, whereas biotype molestus prefers to feed on mammals (Fritz et al. 2015, Osório et al. 2014). Hybrids between both biotypes have a more opportunistic feeding behaviour, and will readily feed on both birds and mammals. Thus, hybrids are considered as important bridge vectors, because they may bridge WNV from birds to humans. Due to the different feeding preferences, the biotypes and hybrids are expected to play different roles in the WNV transmission cycle. Upon introduction of WNV into a new area, biotype pipiens is important for establishment of natural transmission cycles among birds. Once WNV has established and infection rates have increased in the natural (bird) reservoir, especially hybrids and to a lesser extent biotype molestus will become more important for spill-over to humans. In order to assess risks for WNV transmission in a particular area, it is therefore important to differentiate between the $C x$. pipiens biotypes (chapter 7), and to assess variation in their population composition on a temporal and spatial scale (chapters 2 and 3 ). 
Previously, it was considered that there was a clear spatial niche separation between biotypes in northern Europe (Fonseca et al. 2004). Biotype pipiens was considered to occur in aboveground habitats, whereas biotype molestus was considered to be restricted to underground habitats such as flooded basements or tunnels of the underground. Due to this spatial separation between biotypes, hybridization was predicted to be low in northern Europe (Fonseca et al. 2004, Kramer et al. 2008). In southern Europe, hybridization readily occurs because both biotypes inhabit the same aboveground habitats (Gomes et al. 2013a, Gomes et al. 2009). However, the results of this thesis show that this paradigm needs to be revised because both biotype pipiens and molestus were found to occur sympatrically in aboveground locations in The Netherlands (chapters 2 and 3). Consequently, hybridization rates of up to $20 \%$ were found in The Netherlands. These hybridization rates are comparable to rates found in southern European countries such as Italy, Greece, and Portugal (chapter 3; Gomes et al. 2013a, Gomes et al. 2009, Osório et al. 2014). In contrast, hybridization rates were much lower in Sweden (chapter 3). Moreover, a clear shift towards an increasing proportion of biotype pipiens was observed from southern to northern latitudes in Europe (Figure 1). In Italy, the ratio of biotype pipiens to biotype molestus was equal, whereas in Sweden the $C x$. pipiens population was dominated by biotype pipiens (chapter 3). Such shifts in biotype composition were shown to have a strong impact on the $R_{0}$, with relatively higher $R_{0}$ values in populations dominated by biotype pipiens (chapter 7 ). In addition, biotype pipiens contributes most to the $R_{0}$, and thus plays an important role in the initial establishment of WNV (chapter 7). Relatively high proportions of biotype pipiens in the northern European $C x$. pipiens populations may pose an increased risk for WNV establishment, if these populations are competent vectors for WNV. Therefore, biotype composition in northern Europe alone cannot explain the differences in WNV circulation across Europe.

Several studies have now focused on biotype composition in different countries in Europe (Di Luca et al. 2016, Gomes et al. 2013a, Gomes et al. 2009, Osório et al. 2014, Rudolf et al. 2013, Vogels et al. 2016b, Vogels et al. 2015, Zittra et al. 2016). Thus far, most of these studies (including chapters 2 and 3 ) presented the average population composition, by combining data from mosquito collections of varying periods of time. Seasonal patterns in biotype composition may be overlooked when combining data on mosquito collections throughout the season, thereby ignoring potential temporal shifts in the population composition. As peaks in WNV transmission typically follow after seasonal peaks in mosquito abundance (Anderson et al. 2004, Andreadis et al. 2004), it is important to further investigate seasonal changes in the Cx. pipiens biotype composition. 


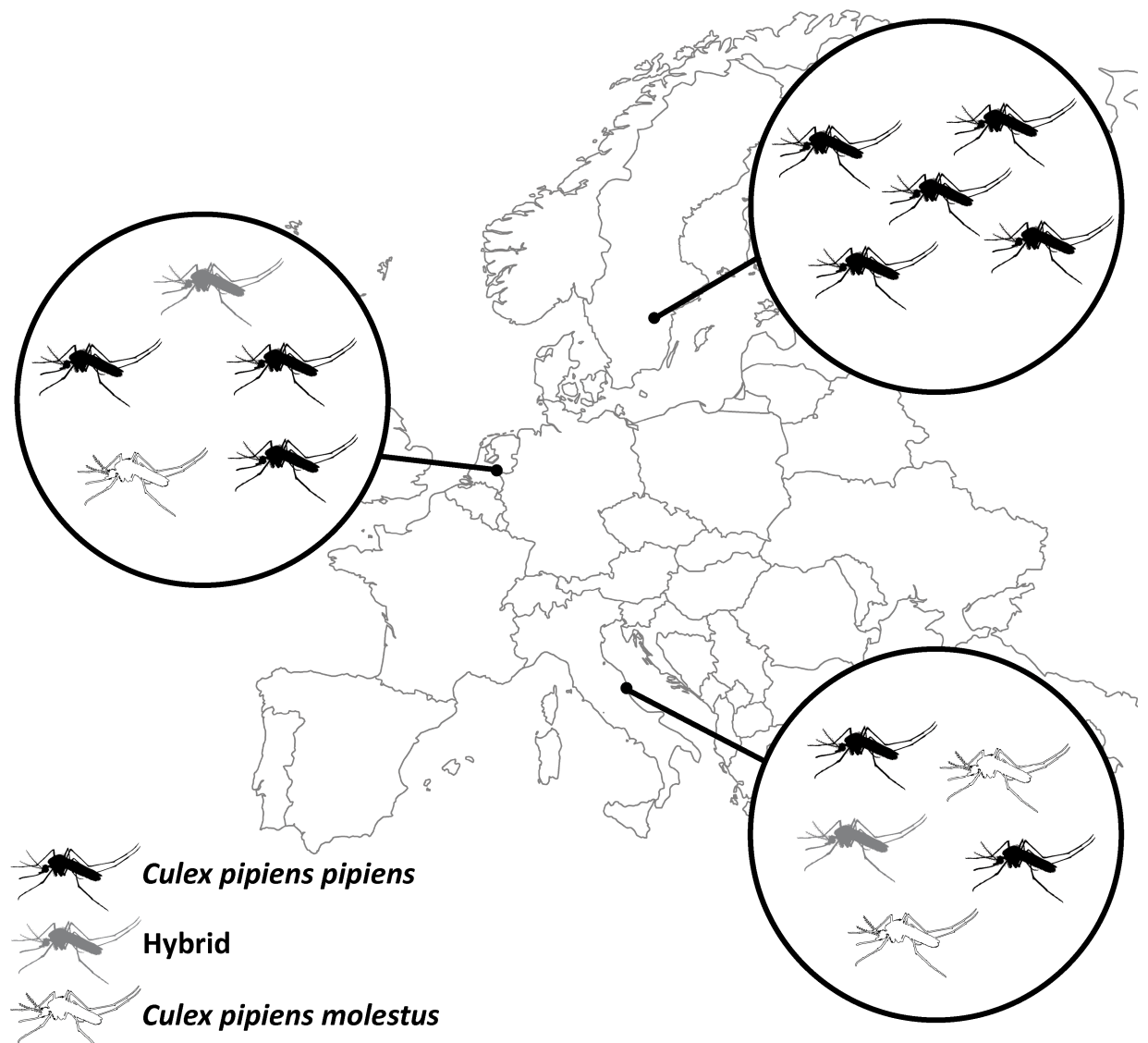

Figure 1: Culex pipiens population composition shifts towards increasing proportions of biotype pipiens at more northern latitudes in Europe. Similar hybridizations rates of up to $15-20 \%$ were found in The Netherlands and Italy. Biotype molestus and hybrids were only found at low proportions $(<10 \%)$ in Sweden.

\section{Mosquito abundance}

Mosquito abundance, i.e. the absolute number of mosquitoes in a given area and time period, is a factor that is not directly included in the calculation of $R_{0}$. Instead, the relation between vector and host abundance is represented by the vector-to-host ratio. Although vector-to-host ratios are convenient for modelling, they do not reflect actual mosquito and host abundance. For instance, a vector-to-host ratio of 10 may in reality result in very different abundances, because areas with 10 mosquitoes and 1 bird host as well as areas with 1000 mosquitoes and 100 bird hosts, both have an equal vector-to-host ratio of 10 . Although abundance is ignored in basic reproduction models (chapter 7), it does play an important role in WNV transmission and the spillover to other hosts such as humans. 
Mosquito abundance is variable and depends on several factors such as habitat, climate, seasonality, and presence of hosts and predators (Chaskopoulou et al. 2016). Relatively high temperatures increase larval development rate (Madder et al. 1983), and therefore southern European countries may face more generations and higher abundance of $C x$. pipiens throughout summer. When comparing abundance of $C x$. pipiens mosquitoes between northern and southern European countries, there indeed appears to be a higher abundance of $C x$. pipiens mosquitoes in southern European areas which experience WNV outbreaks (Chaskopoulou et al. 2016, Ibañez-Justicia et al. 2015). In areas in France, Italy, and Serbia, mean numbers of $C x$. pipiens collected with one trap ranged between approximately 200 700 mosquitoes per night (Chaskopoulou et al. 2016). The highest peak of close to $9,000 \mathrm{Cx}$. pipiens mosquitoes collected with one trap during a single trapping night, based on a 4 year average, was found in rice fields in Greece (Chaskopoulou et al. 2016). In contrast, a total of approximately $7,000 C x$. pipiens mosquitoes were collected during 4 consecutive years of mosquito surveillance in The Netherlands (Ibañez-Justicia et al. 2015). In addition, approximately 5,000 Cx. pipiens were collected during the entire collection period of 1 year in our study in Sweden, The Netherlands, and Italy (chapter 3 ). Interestingly, we collected a greater number of Cx. pipiens mosquitoes from The Netherlands $(n=2,900)$ compared to Italy $(n=$ 2,097 ), despite the same sampling effort. However, no human cases of WNV have yet been reported for the sampling area in Italy, suggesting the necessity of high mosquito abundance for sufficient WNV transmission. Although further confirmation by a direct comparative approach is required, abundance of $C x$. pipiens seems lower in northern European regions without WNV outbreaks compared to regions with WNV outbreaks in southern Europe. Thus, relatively low abundance of $C x$. pipiens may contribute to the absence of WNV outbreaks in northern Europe.

Timing of introduction is another factor that affects the risk of WNV establishment. High abundance of vectors and the introduction of infectious bird hosts need to coincide for establishment of WNV transmission cycles in a particular area (Duijster et al. 2016). Early arrival in spring of long-distance migratory birds, which overwintered in WNV endemic areas in Africa, may result in WNV introduction in northern Europe (Jonzén et al. 2006, Jourdain et al. 2007a). However, abundance of $C x$. pipiens usually peaks later in the season, during the summer months July and August (Chaskopoulou et al. 2016). Therefore, WNV may be introduced into northern Europe before mosquito abundance reaches its peak, leading to local WNV transmission which is not sufficient for spill-over to humans. Indeed, serological evidence of WNV in both migratory and resident birds suggests that WNV can be introduced by migratory birds in northern Europe, and that local transmission might already take place at low rates (Buckley et al. 2006, Buckley et al. 2003, Linke et al. 
2007). Therefore, it is likely that WNV transmission has already taken place in The Netherlands, although no direct evidence of WNV circulation has yet been found (Reusken et al. 2010b, Rockx et al. 2006). Thus, a mismatch between migration of WNV-infected birds towards northern Europe and peak mosquito abundance, may be an additional explanation for the absence of WNV outbreaks in northern Europe.

\section{Vector competence}

The ability of a vector to acquire, maintain, and transmit a pathogen can be defined as vector competence (Kenney \& Brault 2014). Vector competence was hypothesized to be an important factor which may explain the differences in WNV outbreaks between northern and southern Europe. Up to a few years ago, by far the most studies on vector competence for WNV were carried out on American mosquitoes (Ciota et al. 2013a, Dohm et al. 2002, Goddard et al. 2002, Kilpatrick et al. 2010, Kilpatrick et al. 2008, Sardelis et al. 2001, Turell et al. 2005, Turell et al. 2006, Turell et al. 2001a, Vaidyanathan \& Scott 2007), whereas only two studies involved European mosquito species (Balenghien et al. 2008, Balenghien et al. 2007). The American studies showed that vector competence is a variable trait, on the spatial and seasonal scale (Kilpatrick et al. 2010, Vaidyanathan \& Scott 2007). Due to this variation, results obtained from studies on American mosquito species cannot be directly translated to the European situation. Recently, several studies on vector competence of European mosquito species for WNV have been published (Blagrove et al. 2016, Brustolin et al. 2016, Fortuna et al. 2015a, Fortuna et al. 2015b, Fros et al. 2015a, Fros et al. 2015b, Huber et al. 2014, Leggewie et al. 2016, Vogels et al. 2016a). From these studies on European mosquito species, it becomes clear that several mosquito species from across Europe are competent vectors for WNV (chapter 8). However, outcomes of studies are variable, which may be due to variation in experimental protocols, climatic conditions, or intrinsic differences in vector competence between mosquito species or populations.

Our studies with northern European Cx. pipiens mosquitoes have provided evidence of their ability to transmit WNV (chapters 4 and 5; Fros et al. 2015a, Fros et al. 2015b). No differences were found in vector competence between the $C x$. pipiens biotypes, but their vector competence was differentially affected by temperature (chapter 4). Vector competence of biotype pipiens and hybrids increased with higher temperatures, whereas vector competence of biotype molestus remained constant around $10 \%$, within a temperature range of $18{ }^{\circ} \mathrm{C}$ to $28^{\circ} \mathrm{C}$. Maximum transmission rates of up to $33 \%$ were observed for biotype pipiens incubated for 14 days at 28 ${ }^{\circ} \mathrm{C}$. Thus, northern European $\mathrm{Cx}$. pipiens can be considered as competent vectors for WNV. 
The finding that Dutch Cx. pipiens mosquitoes are competent vectors for WNV, does not yet rule out the possibility that their vector competence is relatively lower than vector competence of southern European populations. To identify differences in vector competence across Europe, comparisons can be made between vector competence studies on different European mosquito populations (chapter 8). However, variation in experimental design hampers the ability to draw solid conclusions on intrinsic variation in vector competence. Ruling out variation in experimental design, by making a direct comparison between a northern and southern European $C x$. pipiens population was therefore needed. In a direct comparison between two biotype pipiens populations originating from The Netherlands and Italy, respectively, no differences in vector competence for WNV were found between both populations (chapter 5). Thus, there is no evidence that vector competence differs between the tested Dutch and Italian biotype pipiens populations.

Lack of differences in vector competence between the Dutch and Italian mosquito population contradicts the hypothesis that lowered vector competence may explain the absence of WNV outbreaks in northern Europe. Nevertheless, the hypothesis regarding lowered vector competence of northern European mosquitoes can still not be fully rejected. Although the vector competence of both mosquito populations was similar, temperature was confirmed to be an important factor which can influence vector competence. The effect of temperature on virus replication and transmission has been well established for several arboviruses (Hardy et al. 1983), including WNV (Dohm et al. 2002, Fros et al. 2015a, Fros et al. 2015b, Kilpatrick et al. 2008, Reisen et al. 2006). After incubation for 14 days at a temperature of $18{ }^{\circ} \mathrm{C}$, the average summer temperature of The Netherlands, none (chapter 5) or very few (chapter 4) mosquitoes were able to transmit WNV (Figure 2). In contrast, up to $33 \%$ of Dutch Cx. pipiens biotype pipiens mosquitoes were able to transmit WNV at $28^{\circ} \mathrm{C}$. Thus, not the intrinsic vector competence, but rather temperature is an important limiting factor for WNV transmission in northern European countries such as The Netherlands, which may explain the absence of WNV outbreaks.

It is tempting to extrapolate the results on vector competence of the Dutch and Italian mosquito population to a larger scale, by concluding that there are no differences in vector competence between northern and southern European mosquito populations. However, as previous studies already showed that vector competence can vary on a spatial and temporal scale (Kilpatrick et al. 2010, Vaidyanathan \& Scott 2007), it is likely that local variation exists between mosquito populations in both northern and southern European countries. Thus far, a direct comparison has only been made between one population from northern Europe and one population from southern Europe (chapter 5). Although no differences in vector competence were found between these populations, further studies are needed to confirm these observations 
across a wider spatial scale. In addition, future studies should also focus on the underlying mechanisms of vector competence. In particular, knowledge should be gained on the influence of mosquito barriers, antiviral responses, and microbiome on vector competence, and their relation with temperature (chapter 8 ).

\section{4 days post infection}

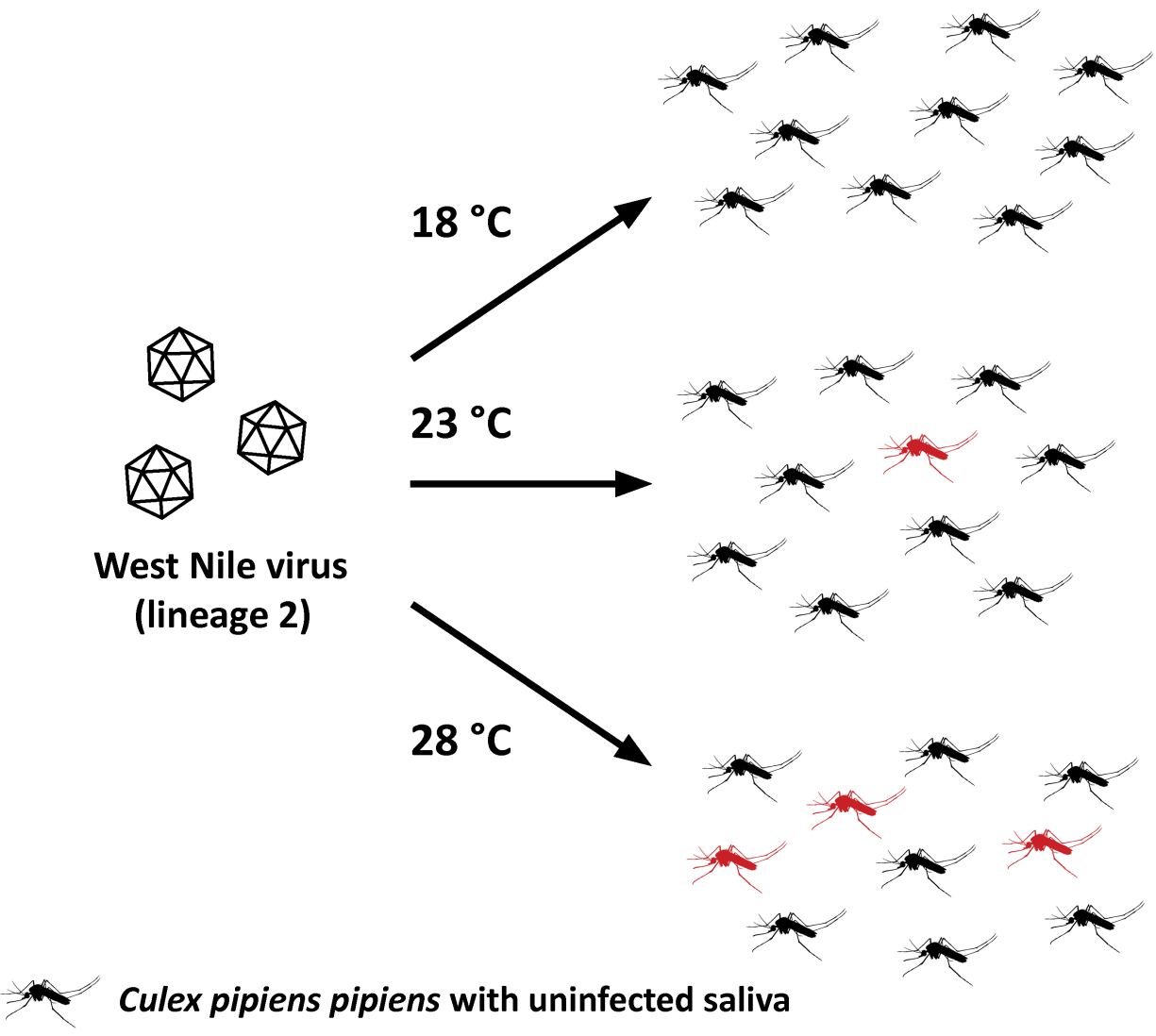

\section{Culex pipiens pipiens with West Nile virus infected saliva}

Figure 2: Dutch Culex pipiens biotype pipiens mosquitoes are competent vectors for West Nile virus, but low temperatures limit transmission. After 14 days of incubation at an average Dutch summer temperature of $18{ }^{\circ} \mathrm{C}$, transmission rates are reduced to $0 \%$. This may explain the current absence of West Nile virus outbreaks in northern European countries such as The Netherlands. 


\section{Extrinsic incubation period}

The extrinsic incubation period (EIP) is defined as the minimum period of time which is needed from initial infection to the moment a pathogen can be transmitted by its vector. Together with survival, the EIP determines for how long a mosquito can spread a pathogen. The EIP for many viruses is dependent on temperature, because temperature can directly influence virus replication (Hardy et al. 1983). For WNV, higher temperatures of up to $37^{\circ} \mathrm{C}$ result in increased virus replication (Andrade et al. 2011), and therefore a relatively higher vector competence and shorter EIP (Kilpatrick et al. 2008, Reisen et al. 2006). Within the range of $18^{\circ} \mathrm{C}$ to $32{ }^{\circ} \mathrm{C}$, the EIP for $C x$. pipiens mosquitoes infected with WNV was found to be shortened from approximately 15 days to 4 days (Kilpatrick et al. 2008).

The vector competence studies carried out in the context of this thesis were all performed at one EIP of 14 days, with a minimum temperature of $18{ }^{\circ} \mathrm{C}$ (chapters 4 and 5). Very few mosquitoes were able to transmit WNV after 14 days incubation at $18^{\circ} \mathrm{C}$. This shows that WNV transmission is much less efficient at low temperatures, but it may also indicate that a longer EIP is required for successful virus transmission (Kilpatrick et al. 2008, Reisen et al. 2006). Mosquitoes that require an EIP which is much longer than 14 days, will not likely have a major contribution to WNV transmission. This is due to a relatively shorter period of the lifetime during which WNV can be transmitted. It would be relevant to determine the minimum EIP as well as the minimum extrinsic incubation temperature for $C x$. pipiens populations across Europe. Thus, potential differences in EIPs between northern and southern European mosquito populations deserve further attention in future research.

\section{Survival}

Arboviruses cause diseases in vertebrate hosts, but are typically tolerated by their arthropod vectors. Tolerance is a strategy that reduces the negative impact of infection on fitness, but unlike resistance, it does not result in complete clearance of viral infection (Ayres \& Schneider 2012, Medzhitov et al. 2012). WNV was shown to replicate up to a maximum $50 \%$ tissue culture infectious dose of approximately $10^{8} \mathrm{TCID}_{50} / \mathrm{ml}$ inside the mosquito body (chapters 4 and 5), without causing significant negative consequences on mosquito survival (chapter 6 ). These findings are supported by other studies on the survival of $C x$. pipiens and $C x$. tarsalis after infection with WNV (Ciota et al. 2013b, Styer et al. 2007).

Only if a mosquito survives longer than the EIP, the mosquito can contribute to transmission of the pathogen (Figure 3). Thus, survival during the EIP is crucial for WNV transmission. Although higher temperatures have a positive effect on vector 
competence of $C x$. pipiens for WNV and the EIP (chapters 4 and 5; Dohm et al. 2002, Fros et al. 2015a, Fros et al. 2015b, Kilpatrick et al. 2008), they also negatively affect survival (Ciota et al. 2014). No studies have yet investigated the interaction between temperature and WNV infection on survival of $C x$. pipiens. This may reveal relevant findings which should be included in WNV risk assessment, because survival plays an important role in the duration of the infectious lifetime of vectors. The cooler northern European climate will likely result in relatively prolonged mosquito survival, but it also extends the duration of the EIP (Figure 3). Consequently, the duration of the infectious lifetime may be severely reduced, which can possibly explain the lack of WNV outbreaks in northern Europe. Investigation of survival and EIP of mosquitoes across Europe, especially under field conditions with fluctuating temperatures, is needed to make better predictions of the WNV transmission potential.

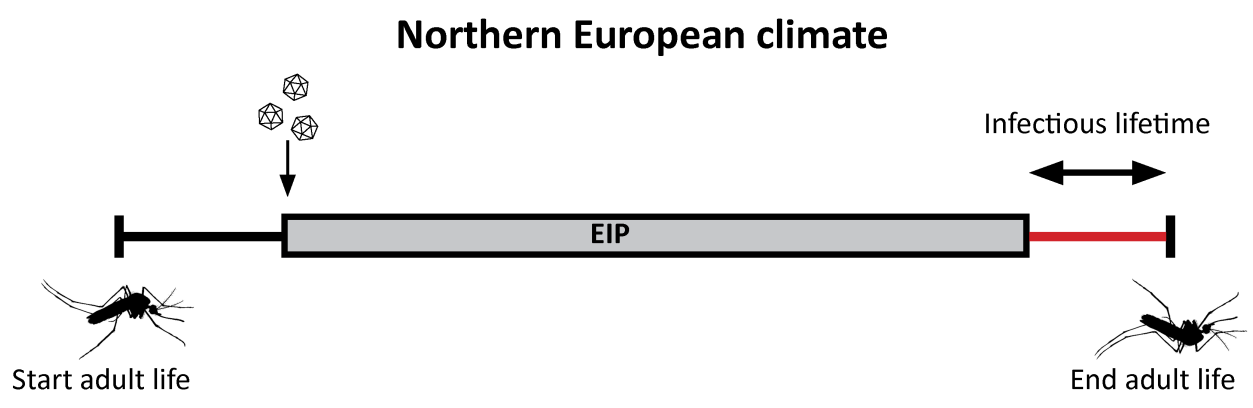

\section{Southern European climate}

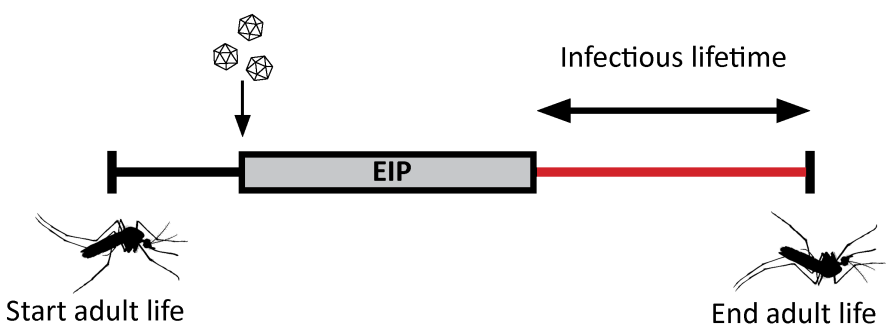

Figure 3: The northern European climate may decrease the infectious lifetime of Culex pipiens mosquitoes infected with West Nile virus. The relatively cooler northern European climate results in longer survival and an extended extrinsic incubation period (EIP), whereas the warmer southern European climate results in shorter survival and a shortened EIP. Consequently, low temperatures can lead to a shortened infectious lifetime which may not be sufficient for efficient West Nile virus transmission. 


\section{Biting behaviour}

Biting behaviour is crucial for transmission of WNV, because the transmission cycle can only be completed if a mosquito transmits or picks up WNV when feeding on a bird. Differences in host preferences of the $C x$. pipiens biotypes were shown to be important for their contribution to the $R_{0}$ (chapter 7 ). However, not only host preference, but also the frequency with which a mosquito bites a host (i.e. biting rate), is important from the perspective of the virus. At higher temperatures the length of the mosquito's gonotrophic cycle is shorter, which results in a shorter time between consecutive blood meals (Platonov et al. 2008). Thus, the southern European climate may favour a higher biting rate which can be beneficial for WNV transmission. It would also be beneficial for virus transmission, if viruses themselves can maximize their transmission potential by increasing the biting rate of their vector (Hurd 2003, Lefèvre \& Thomas 2008, van Houte et al. 2013). Interestingly, I found that WNV reduced the host-seeking response of $C x$. pipiens, and did not interfere with the flight activity or ability to blood feed (chapter 6). Taken together, infection with WNV leads to a decreased frequency of host feeding, which seems detrimental for WNV transmission. Here, a WNV dose-dependent effect may explain the ongoing WNV transmission in the field (Figure 4). In this case, inoculation of mosquitoes with high WNV titers leads to efficient WNV dissemination and, thus, high transmission rates (chapters 4 and 5), but also to a reduced host-seeking response (chapter 6). In contrast, inoculation of mosquitoes with lower WNV titers may not result in efficient dissemination and, thus, relatively lower transmission rates (Anderson et al. 2010). I hypothesize that due to lower WNV titers in the mosquito body, the chance of infection of secondary organs such as the brain is lower, and therefore I do not expect a strong reduction in the host-seeking response (Figure 4). These opposing drivers will likely result in a transmission optimum at intermediate WNV titers in the mosquito body, which may explain the ongoing WNV transmission in the field. More research is needed to fully understand the interaction between WNV and the hostseeking and biting behaviour of its mosquito vector. Investigating the host-seeking response of mosquitoes inoculated with varying WNV doses or determination of WNV titers in field-collected mosquitoes would be needed to test the hypothesis that the mosquito's host-seeking response is dependent on viral dose. In addition, further investigation of infection of the mosquito brain by WNV is needed to unravel the underlying mechanisms of the decreased host-seeking response. 


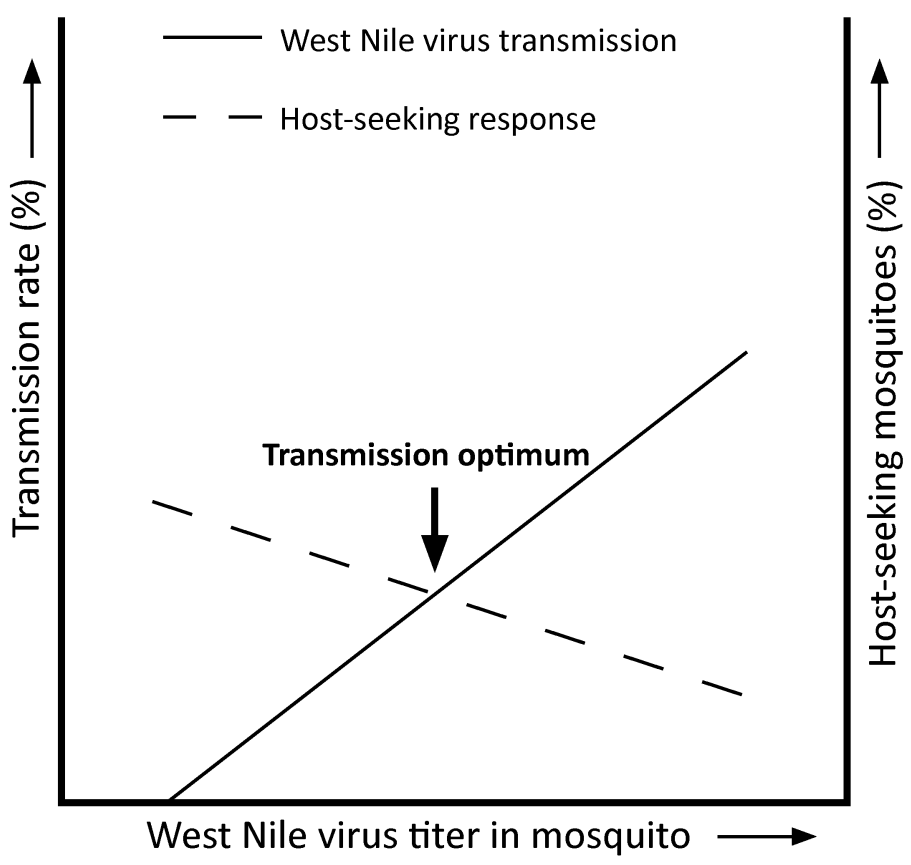

Figure 4: Visualisation of hypothesis on the dose-dependent effect of West Nile virus titers in the mosquito body on transmission and the mosquito's host-seeking response. Opposing drivers are expected to result in optimal West Nile virus transmission at intermediate virus titers in the mosquito body. This may explain ongoing West Nile virus transmission in the field, despite the lowered host-seeking response found for mosquitoes infected with relatively high West Nile virus titers.

Implications of the decreased host-seeking response of $C x$. pipiens infected with WNV were not included in our $R_{0}$ model (chapter 7). Thus far, only the effect of WNV infection on the behaviour of biotype pipiens has been studied. Therefore, it was not yet possible to fully integrate the consequences of WNV infection on biting activity of the Cx. pipiens biotypes and hybrids, in the newly developed $R_{0}$ model (chapter 7). However, consequences of reduced host-seeking behaviour will likely lower the outcome of the $R_{0}$ model, thereby reducing the likelihood of WNV establishment. The question remains to what extent similar reductions in the host-seeking response can be observed for other combinations of WNV strains and Cx. pipiens populations, or even at a wider scale with other arboviruses and mosquito species. Thus, investigating the effect of arbovirus infection on mosquito host-seeking behaviour presents an exciting field for future research.

Priority should also be given to research focusing on the preference of European biotype pipiens mosquitoes for specific bird species. Most studies have thus far determined host preferences of American Cx. pipiens mosquitoes (Fritz et al. 2015, Hamer et al. 2009, Kilpatrick et al. 2006). Studies in the USA identified the American 
robin (Turdus migratorius) as the main host, due to high feeding preference of the main WNV vectors for this particular bird species (Hamer et al. 2009, Kilpatrick et al. 2006). Similar studies focusing on specific preferences of European $C x$. pipiens mosquitoes remain scarce (Muñoz et al. 2012, Osório et al. 2012, Rizzoli et al. 2015a). In order to streamline future research on the role of European bird hosts in the WNV transmission cycle, I strongly suggest to first identify bird species which are highly preferred by $C x$. pipiens mosquitoes in the wild. This can, for instance, be done by blood meal analysis of engorged $C x$. pipiens mosquitoes collected from the field (Osório et al. 2012, Rizzoli et al. 2015a), or by testing host preference for specific bird species in an olfactometer under controlled laboratory conditions (chapter 6; Fritz et al. 2015). Once candidate bird species have been identified, the second step would be to test these bird species for their susceptibility to WNV in the laboratory. Identification of key bird species in the European WNV transmission cycle is highly important for targeted control measures against WNV.

\section{Risk of West Nile virus transmission in northern Europe}

When comparing all mosquito-related factors that play a role in the risk for WNV establishment, it becomes clear that temperature has the strongest influence on several mosquito-related factors. Thus, low temperature is an important factor which may explain the lack of WNV outbreaks in northern Europe. To summarize, temperature influences three main mosquito-related factors that are important for WNV transmission: mosquito abundance, vector competence, and the mosquito's infectious lifetime (Figure 5). My thesis has clearly shown that competent vectors are present in northern European countries such as The Netherlands. The northern European $C x$. pipiens biotype pipiens population from The Netherlands had a similar intrinsic vector competence compared to a southern European population from Italy. However, the colder northern European climate keeps mosquito populations at relatively low abundance, and poses a major limitation for WNV transmission by lowering vector competence, and by extending the EIP. The combination of these factors most likely explains the current absence of WNV outbreaks in northern Europe. 


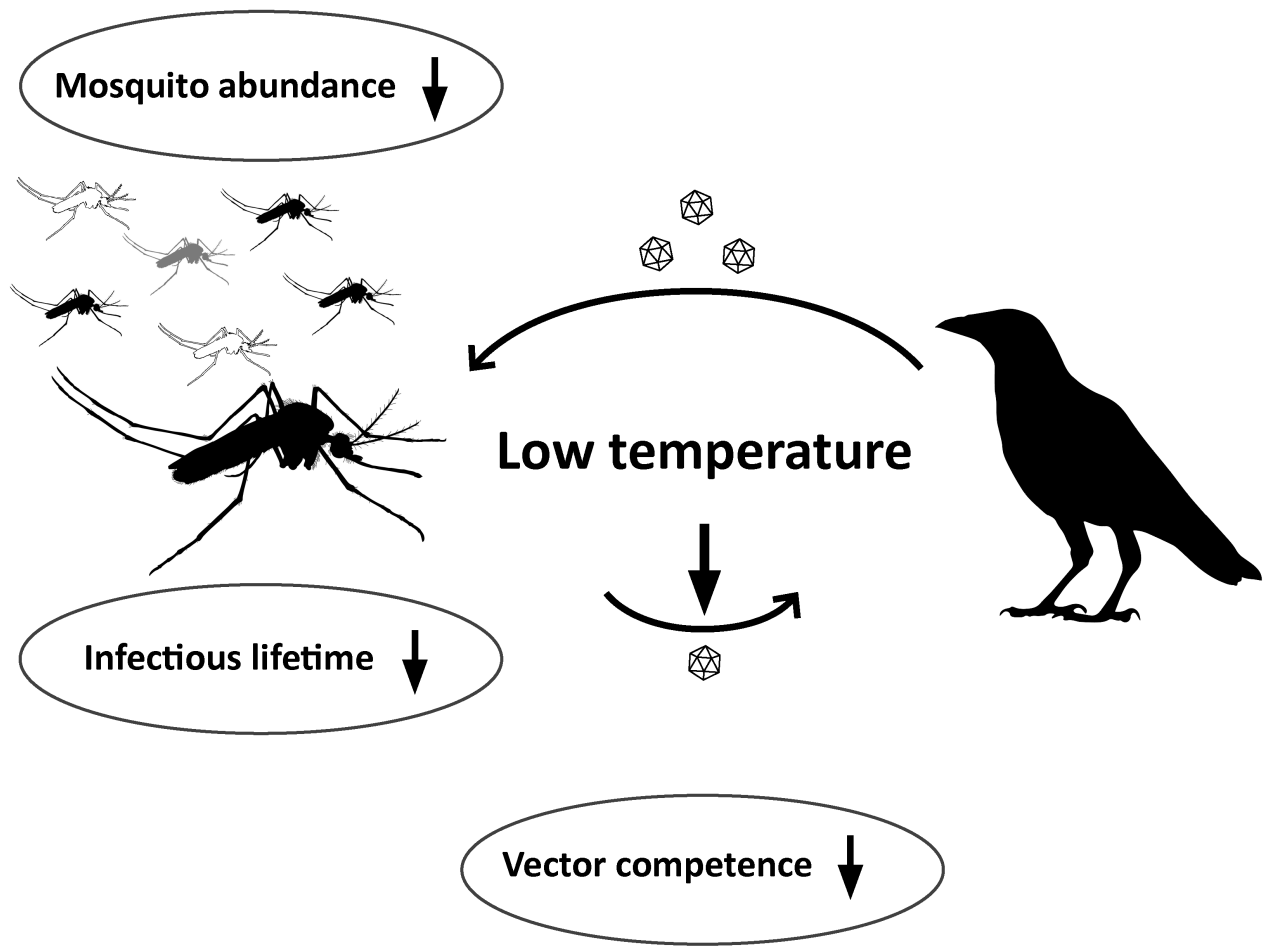

Figure 5: Mosquito-related factors which limit West Nile virus transmission at low temperatures in northern Europe. Low temperature negatively influences mosquito abundance, vector competence, and the infectious lifetime of mosquitoes. Together these factors keep West Nile virus transmission in northern Europe at low levels, which most likely explains the lack of West Nile virus outbreaks in humans. Colours of mosquitoes represent the different Culex pipiens biotypes and hybrids, with biotype pipiens: black, biotype molestus: white, and hybrids: grey.

Global warming will likely favour the northward movement of WNV and other related arboviruses in Europe. During the relatively warm autumn of 2016, Usutu virus, a flavivirus closely related to WNV, was detected for the first time in The Netherlands (Cadar et al. 2017, Rijks et al. 2016). Due to the similar ecology of WNV and Usutu virus, I consider the circulation of Usutu virus in The Netherlands as a prelude for WNV transmission (Fros et al. 2015b). When considering the results of my thesis in the light of climate change predictions of more frequent and prolonged periods of heat, northern Europe should be prepared for future WNV transmission. However, outcomes of this thesis also show that it is unlikely that major WNV outbreaks will occur in the near future. Nevertheless, I strongly advise the implementation of WNV surveillance tools in northern European countries, including The Netherlands. Early detection of WNV will aid in the preparedness for WNV transmission in northern European countries (chapter 8). Various active and passive surveillance tools are available, focusing on detection of WNV in mosquito vectors, (dead) bird hosts, or 
in suspected cases of encephalitis in humans or equines (Engler et al. 2013). Active surveillance by testing for presence of WNV in mosquitoes or birds often results in low detection rates, even during WNV outbreaks (Engler et al. 2013). To my opinion, passive surveillance of dead birds or investigation of suspected human and equine cases of encephalitis seems most effective for northern Europe (Rockx et al. 2006). Current initiatives such as Eco-Alert, aimed at the development of an infrastructure for early detection of arboviruses, will aid in a proper response to the introduction of arboviruses in The Netherlands (Cleton et al. 2016, Rijks et al. 2016). In addition, current mosquito monitoring programs which are operated by the National Centre for Monitoring of Vectors, provide important insights into the presence and abundance of both endemic and exotic mosquito species in The Netherlands (Ibañez-Justicia et al. 2015). These insights are important for targeted control of newly introduced exotic mosquito species, but may also become essential for possible future control of endemic WNV vectors such as $C x$. pipiens. Furthermore, increased awareness of WNV among the general public and health professionals in northern Europe is required. One way to increase awareness among the general public is by actively involving citizens in mosquito research, such as the Dutch citizen science project "Muggenradar" (chapter 2). Such forms of citizen science do not only increase knowledge and awareness of the general public on mosquitoes and their possible risks, but are also a valuable alternative tool for mosquito surveillance. Thus, surveillance and awareness are key in order to be prepared for a new era in which mosquitoes in northern Europe are not only associated with nuisance, but also with transmission of arboviruses such as West Nile virus.

\section{Acknowledgements}

I thank Marcel Dicke and Sander Koenraadt for providing comments on an earlier version of this chapter. 

References 
Adelman, Z.N., Anderson, M.A.E., Wiley, M.R., Murreddu, M.G., Samuel, G.H., Morazzani, E.M., Myles, K.M. 2013. Cooler temperatures destabilize RNA interference and increase susceptibility of disease vector mosquitoes to viral infection. PLoS Neglected Tropical Diseases 7:e2239.

Allan, S.A., Bernier, U.R., Kline, D.L. 2010. Laboratory evaluation of lactic acid on attraction of Culex spp. (Diptera: Culicidae). Journal of Vector Ecology 35:318-324.

Almeida, A.P.G., Galão, R.P., Sousa, C.A., Novo, M.T., Parreira, R., Pinto, J., Piedade, J., Esteves, A. 2008. Potential mosquito vectors of arboviruses in Portugal: species, distribution, abundance and West Nile infection. Transactions of the Royal Society of Tropical Medicine and Hygiene 102:823-832.

Alto, B.W. \& Bettinardi, D. 2013. Temperature and dengue virus infection in mosquitoes: independent effects on the immature and adult stages. The American Journal of Tropical Medicine and Hygiene 88:497-505.

Amraoui, F., Tijane, M., Sarih, M., Failloux, A.B. 2012. Molecular evidence of Culex pipiens form molestus and hybrids pipiens/molestus in Morocco, North Africa. Parasites \& Vectors 5:83.

Anderson, J.F., Andreadis, T.G., Main, A.J., Kline, D.L. 2004. Prevalence of West Nile virus in tree canopy-inhabiting Culex pipiens and associated mosquitoes. The American Journal of Tropical Medicine and Hygiene 71:112-119.

Anderson, J.F. \& Main, A.J. 2006. Importance of vertical and horizontal transmission of West Nile virus by Culex pipiens in the northeastern United States. The Journal of Infectious Diseases 194:1577-1579.

Anderson, J.F., Main, A.J., Cheng, G., Ferrandino, F.J., Fikrig, E. 2012. Horizontal and vertical transmission of West Nile virus genotype NY99 by Culex salinarius and genotypes NY99 and WN02 by Culex tarsalis. The American Journal of Tropical Medicine and Hygiene 86:134-139.

Anderson, R.M. \& May, R.M. 1990. Immunisation and herd immunity. The Lancet 335:641645.

Anderson, S.L., Richards, S.L., Tabachnick, W.J., Smartt, C.T. 2010. Effects of West Nile virus dose and extrinsic incubation temperature on temporal progression of vector competence in Culex pipiens quinquefasciatus. Journal of the American Mosquito Control Association 26:103-107.

Andrade, C.C., Maharaj, P.D., Reisen, W.K., Brault, A.C. 2011. North American West Nile virus genotype isolates demonstrate differential replicative capacities in response to temperature. Journal of General Virology 92:2523-2533.

Andreadis, T.G., Anderson, J.F., Vossbrinck, C.R., Main, A.J. 2004. Epidemiology of West Nile virus in Connecticut: A five-year analysis of mosquito data 1999-2003. Vector-Borne and Zoonotic Diseases 4:360-378.

Arbouzova, N.I. \& Zeidler, M.P. 2006. JAK/STAT signalling in Drosophila: insights into conserved regulatory and cellular functions. Development 133:2605-2616.

Ayres, J.S. \& Schneider, D.S. 2012. Tolerance of infections. Annual Review of Immunology 30:271-294.

Bae, H.-G., Nitsche, A., Teichmann, A., Biel, S.S., Niedrig, M. 2003. Detection of yellow fever virus: a comparison of quantitative real-time PCR and plaque assay. Journal of Virological Methods 110:185-191. 
Bakonyi, T., Ivanics, É., Erdélyi, K., Ursu, K., Ferenczi, E., Weissenbock, H., Nowotny, N. 2006. Lineage 1 and 2 strains of encephalitic West Nile virus, central Europe. Emerging Infectious Diseases 12:618-623.

Balenghien, T., Fouque, F., Sabatier, P., Bicout, D.J. 2006. Horse-, bird-, and human-seeking behavior and seasonal abundance of mosquitoes in a West Nile virus focus of southern France. Journal of Medical Entomology 43:936-946.

Balenghien, T., Vazeille, M., Grandadam, M., Schaffner, F., Zeller, H., Reiter, P., Sabatier, P., Fouque, F., Bicout, D.J. 2008. Vector competence of some French Culex and Aedes mosquitoes for West Nile virus. Vector-Borne and Zoonotic Diseases 8:589-595.

Balenghien, T., Vazeille, M., Reiter, P., Schaffner, F., Zeller, H., Bicout, D.J. 2007. Evidence of laboratory vector competence of Culex modestus for West Nile virus. Journal of the American Mosquito Control Association 23:233-236.

Bardos, V., Adamcová, J., Dedei, S., Gjini, N., Rosický, B., Simkova, A. 1959. Neutralizing antibodies against some neurotropic viruses determined in human sera in Albania. Journal of Hygiene, Epidemiology, Microbiology and Immunology 3:277-82.

Barón, O.L., Ursic-Bedoya, R.J., Lowenberger, C.A., Ocampo, C.B. 2010. Differential gene expression from midguts of refractory and susceptible lines of the mosquito, Aedes aegypti, infected with Dengue-2 virus. Journal of Insect Science 10:41.

Bartholomay, L.C., Waterhouse, R.M., Mayhew, G.F., Campbell, C.L., Michel, K., Zou, Z., Ramirez, J.L., Das, S., Alvarez, K., Arensburger, P., Bryant, B., Chapman, S.B., Dong, Y., Erickson, S.M., Karunaratne, S.H.P.P., Kokoza, V., Kodira, C.D., Pignatelli, P., Shin, S.W., Vanlandingham, D.L., Atkinson, P.W., Birren, B., Christophides, G.K., Clem, R.J., Hemingway, J., Higgs, S., Megy, K., Ranson, H., Zdobnov, E.M., Raikhel, A.S., Christensen, B.M., Dimopoulos, G., Muskavitch, M.A.T. 2010. Pathogenomics of Culex quinquefasciatus and meta-analysis of infection responses to diverse pathogens. Science 330:88-90.

Becker, N., Jöst, A., Weitzel, T. 2012. The Culex pipiens complex in Europe. Journal of the American Mosquito Control Association 28:53-67.

Becker, N., Petrić, D., Boase, C., Lane, J., Zgomba, M., Dahl, C., Kaiser, A. 2010. Mosquitoes and their control. Springer, Heidelberg.

Beniston, M., Stephenson, D.B., Christensen, O.B., Ferro, C.A.T., Frei, C., Goyette, S., Halsnaes, K., Holt, T., Jylhä, K., Koffi, B., Palutikof, J., Schöll, R., Semmler, T., Woth, K. 2007. Future extreme events in European climate: an exploration of regional climate model projections. Climatic Change 81:71-95.

Blagrove, M.S.C., Sherlock, K., Chapman, G.E., Impoinvil, D.E., McCall, P.J., Medlock, J.M., Lycett, G., Solomon, T., Baylis, M. 2016. Evaluation of the vector competence of a native UK mosquito Ochlerotatus detritus (Aedes detritus) for dengue, chikungunya and West Nile viruses. Parasites \& Vectors 9:1-6.

Blair, C.D. 2011. Mosquito RNAi is the major innate immune pathway controlling arbovirus infection and transmission. Future Microbiology 6:265-277.

Blanc, S. \& Michalakis, Y. 2016. Manipulation of hosts and vectors by plant viruses and impact of the environment. Current Opinion in Insect Science 16:36-43.

Bowen, R.A. \& Nemeth, N.M. 2007. Experimental infections with West Nile virus. Current Opinion in Infectious Diseases 20:293-297.

Brackney, D.E., Beane, J.E., Ebel, G.D. 2009. RNAi targeting of West Nile virus in mosquito midguts promotes virus diversification. PLoS Pathogens 5:e1000502. 
Braks, M.A.H. \& Takken, W. 1999. Incubated human sweat but not fresh sweat attracts the malaria mosquito Anopheles gambiae sensu stricto. Journal of Chemical Ecology 25:663-672.

Brand, S.P.C., Rock, K.S., Keeling, M.J. 2016. The interaction between vector life history and short vector life in vector-borne disease transmission and control. PLoS Computational Biology 12:e1004837.

Brustolin, M., Talavera, S., Santamaría, C., Rivas, R., Pujol, N., Aranda, C., Marquès, E., Valle, M., Verdún, M., Pagès, N., Busquets, N. 2016. Culex pipiens and Stegomyia albopicta (=Aedes albopictus) populations as vectors for lineage 1 and 2 West Nile virus in Europe. Medical and Veterinary Entomology 30:166-173.

Buckley, A., Dawson, A., Gould, E.A. 2006. Detection of seroconversion to West Nile virus, Usutu virus and Sindbis virus in UK sentinel chickens. Virology Journal 3:71.

Buckley, A., Dawson, A., Moss, S.R., Hinsley, S.A., Bellamy, P.E., Gould, E.A. 2003. Serological evidence of West Nile virus, Usutu virus and Sindbis virus infection of birds in the UK. Journal of General Virology 84:2807-2817.

Byrne, K. \& Nichols, R.A. 1999. Culex pipiens in London Underground tunnels: differentiation between surface and subterranean populations. Heredity 82:7-15.

Cadar, D., Lühken, R., van der Jeugd, H., Garigliany, M., Ziegler, U., Keller, M., Lahoreau, J., Lachmann, L., Becker, N., Kik, M. 2017. Widespread activity of multiple lineages of Usutu virus, western Europe, 2016. Eurosurveillance 22:30452.

Calistri, P., Giovannini, A., Hubalek, Z., Lonescu, A., Monaco, F., Savini, G., Lelli, R. 2010a. Epidemiology of West Nile in Europe and in the Mediterranean Basin. The Open Virology Journal 4:29-37.

Calistri, P., Giovannini, A., Savini, G., Monaco, F., Bonfanti, L., Ceolin, C., Terregino, C., Tamba, M., Cordioli, P., Lelli, R. 2010b. West Nile virus transmission in 2008 in NorthEastern Italy. Zoonoses and Public Health 57:211-219.

Caswell, H. 2001. Matrix population models: construction, analysis, and interpretation. Sinauer Associates, Sunderland.

Cator, L.J., George, J., Blanford, S., Murdock, C.C., Baker, T.C., Read, A.F., Thomas, M.B. 2013. 'Manipulation' without the parasite: altered feeding behaviour of mosquitoes is not dependent on infection with malaria parasites. Proceedings of the Royal Society B: Biological Sciences 280:20130711.

CDC. 2012a. Mosquito species in which West Nile virus has been detected, United States, 1999-2012. Centers for Disease Control and Prevention. https://www.cdc.gov/westnile/ resources/pdfs/mosquitospecies1999-2012.pdf.

CDC. 2012b. Species of dead birds in which West Nile virus has been detected, United States, 1999-2012. Centers for Disease Control and Prevention. https://www.cdc.gov/ westnile/resources/pdfs/birdspecies1999-2012.pdf.

CDC. 2015. West Nile virus disease cases and deaths reported to CDC by year and clinical presentation, 1999-2015. Centers for Disease Control and Prevention. https://www. cdc.gov/westnile/statsmaps/cummapsdata.html.

Chamberlain, R.W. \& Sudia, W.D. 1961. Mechanism of transmission of viruses by mosquitoes. Annual Review of Entomology 6:371-390.

Chancey, C., Grinev, A., Volkova, E., Rios, M. 2015. The global ecology and epidemiology of West Nile virus. BioMed Research International 2015:20. 
Chaskopoulou, A., L'Ambert, G., Petric, D., Bellini, R., Zgomba, M., Groen, T.A., Marrama, L., Bicout, D.J. 2016. Ecology of West Nile virus across four European countries: review of weather profiles, vector population dynamics and vector control response. Parasites \& Vectors 9:482.

Chevillon, C., Eritja, R., Pasteur, N., Raymond, M. 1995. Commensalism, adaptation and gene flow: mosquitoes of the Culex pipiens complex in different habitats. Genetics Research 66:147-157.

Chouin-Carneiro, T., Vega-Rua, A., Vazeille, M., Yebakima, A., Girod, R., Goindin, D., Dupont-Rouzeyrol, M., Lourenço-de-Oliveira, R., Failloux, A.B. 2016. Differential susceptibilities of Aedes aegypti and Aedes albopictus from the Americas to Zika Virus. PLoS Neglected Tropical Diseases 10:e0004543.

Choy, M.M., Ellis, B.R., Ellis, E.M., Gubler, D.J. 2013. Comparison of the mosquito inoculation technique and quantitative real time polymerase chain reaction to measure dengue virus concentration. The American Journal of Tropical Medicine and Hygiene 89:10011005.

Ciota, A.T., Chin, P.A., Kramer, L.D. 2013a. The effect of hybridization of Culex pipiens complex mosquitoes on transmission of West Nile virus. Parasites \& Vectors 6:305.

Ciota, A.T., Ehrbar, D.J., Matacchiero, A.C., Van Slyke, G.A., Kramer, L.D. 2013b. The evolution of virulence of West Nile virus in a mosquito vector: implications for arbovirus adaptation and evolution. BMC Evolutionary Biology 13:71.

Ciota, A.T., Lovelace, A.O., Ngo, K.A., Le, A.N., Maffei, J.G., Franke, M.A., Payne, A.F., Jones, S.A., Kauffman, E.B., Kramer, L.D. 2007. Cell-specific adaptation of two flaviviruses following serial passage in mosquito cell culture. Virology 357:165-174.

Ciota, A.T., Matacchiero, A.C., Kilpatrick, A.M., Kramer, L.D. 2014. The effect of temperature on life history traits of Culex mosquitoes. Journal of Medical Entomology 51:55-62.

Clem, R.J. 2016. Arboviruses and apoptosis: the role of cell death in determining vector competence. Journal of General Virology 97:1033-1036.

Cleton, N.B., van Maanen, K., Bergervoet, S.A., Bon, N., Beck, C., Godeke, G.J., Lecollinet, S., Bowen, R., Lelli, D., Nowotny, N., Koopmans, M.P.G., Reusken, C.B.E.M. 2016. A serological protein microarray for detection of multiple cross-reactive flavivirus infections in horses for veterinary and public health surveillance. Transboundary and Emerging Diseases

Colpitts, T.M., Cox, J., Vanlandingham, D.L., Feitosa, F.M., Cheng, G., Kurscheid, S., Wang, P., Krishnan, M.N., Higgs, S., Fikrig, E. 2011. Alterations in the Aedes aegypti transcriptome during infection with West Nile, dengue and yellow fever viruses. PLoS Pathogens 7:e1002189.

Cornel, A.J., Jupp, P.G., Blackburn, N.K. 1993. Environmental temperature on the vector competence of Culex univittatus (Diptera: Culicidae) for West Nile virus. Journal of Medical Entomology 30:449-456.

Cornet, S., Nicot, A., Rivero, A., Gandon, S. 2013. Malaria infection increases bird attractiveness to uninfected mosquitoes. Ecology Letters 16:323-329.

Cressler, C.E., Nelson, W.A., Day, T., McCauley, E. 2014. Disentangling the interaction among host resources, the immune system and pathogens. Ecology Letters 17:284-293.

da Cunha Sais, T., de Moraes, R.M., Ribolla, P.E., de Bianchi, A.G., Marinotti, O., Bijovsky, A.T. 2003. Morphological aspects of Culex quinquefasciatus salivary glands. Arthropod Structure \& Development 32:219-226. 
Danforth, M.E., Reisen, W.K., Barker, C.M. 2016. The impact of cycling temperature on the transmission of West Nile virus. Journal of Medical Entomology 53:681-686.

Dauphin, G., Zientara, S., Zeller, H., Murgue, B. 2004. West Nile: worldwide current situation in animals and humans. Comparative Immunology, Microbiology and Infectious Diseases 27:343-355.

Davis, C.T., Ebel, G.D., Lanciotti, R.S., Brault, A.C., Guzman, H., Siirin, M., Lambert, A., Parsons, R.E., Beasley, D.W.C., Novak, R.J., Elizondo-Quiroga, D., Green, E.N., Young, D.S., Stark, L.M., Drebot, M.A., Artsob, H., Tesh, R.B., Kramer, L.D., Barrett, A.D.T. 2005. Phylogenetic analysis of North American West Nile virus isolates, 20012004: evidence for the emergence of a dominant genotype. Virology 342:252-265.

de Kroon, H., Plaisier, A., van Groenendael, J., Caswell, H. 1986. Elasticity: The relative contribution of demographic parameters to population growth rate. Ecology 67:14271431.

De Moraes, C.M., Stanczyk, N.M., Betz, H.S., Pulido, H., Sim, D.G., Read, A.F., Mescher, M.C. 2014. Malaria-induced changes in host odors enhance mosquito attraction. Proceedings of the National Academy of Sciences 111:11079-11084.

Dekker, T., Geier, M., Cardé, R.T. 2005. Carbon dioxide instantly sensitizes female yellow fever mosquitoes to human skin odours. Journal of Experimental Biology 208:29632972.

Del Amo, J., Llorente, F., Pérez-Ramirez, E., Soriguer, R.C., Figuerola, J., Nowotny, N., Jiménez-Clavero, M.A. 2014. Experimental infection of house sparrows (Passer domesticus) with West Nile virus strains of lineages 1 and 2. Veterinary Microbiology 172:542-547.

Di Luca, M., Toma, L., Boccolini, D., Severini, F., La Rosa, G., Minelli, G., Bongiorno, G., Montarsi, F., Arnoldi, D., Capelli, G., Rizzoli, A., Romi, R. 2016. Ecological distribution and CQ11 genetic structure of Culex pipiens complex (Diptera: Culicidae) in Italy. PLoS One 11:e0146476.

Diekmann, O. \& Heesterbeek, J.A.P. 2000. Mathematical epidemiology of infectious diseases: model building, analysis and interpretation. John Wiley \& Sons, New York.

Diekmann, O., Heesterbeek, J.A.P., Metz, J.A.J. 1990. On the definition and the computation of the basic reproduction ratio $\mathrm{RO}$ in models for infectious diseases in heterogeneous populations. Journal of Mathematical Biology 28:365-382.

Dietz, K. 1993. The estimation of the basic reproduction number for infectious diseases. Statistical Methods in Medical Research 2:23-41.

DiMenna, M.A., Bueno, R., Parmenter, R.R., Norris, D.E., Sheyka, J.M., Molina, J.L., LaBeau, E.M., Hatton, E.S., Glass, G.E. 2006. Comparison of mosquito trapping method efficacy for West Nile virus surveillance in New Mexico. Journal of the American Mosquito Control Association 22:246-253.

Dodson, B.L., Hughes, G.L., Paul, O., Matacchiero, A.C., Kramer, L.D., Rasgon, J.L. 2014. Wolbachia enhances West Nile virus (WNV) infection in the mosquito Culex tarsalis. PLoS Neglected Tropical Diseases 8:e2965.

Dohm, D.J., O'Guinn, M.L., Turell, M.J. 2002. Effect of environmental temperature on the ability of Culex pipiens (Diptera: Culicidae) to transmit West Nile virus. Journal of Medical Entomology 39:221-225.

Dohm, D.J. \& Turell, M.J. 2001. Effect of incubation at overwintering temperatures on the replication of West Nile virus in New York Culex pipiens (Diptera : Culicidae). Journal of Medical Entomology 38:462-464. 
Duijster, J.W., Stroo, C.J., Braks, M.A.H. 2016. Birds, mosquitoes and West Nile virus: little risk of West Nile fever in the Netherlands. Nederlands Tijdschrift voor Geneeskunde 160:A9899-A9899.

Ebel, G.D., Carricaburu, J., Young, D., Bernard, K.A., Kramer, L.D. 2004. Genetic and phenotypic variation of West Nile virus in New York, 2000-2003. The American Journal of Tropical Medicine and Hygiene 71:493-500.

ECDC. 2016. West Nile fever data Europe 2010-2016. European Centre for Disease Prevention and Control. http://ecdc.europa.eu/en/healthtopics/west_nile_fever/WestNile-fever-maps/Pages/historical-data.aspx.

Eidson, M., Kramer, L., Stone, W., Hagiwara, Y., Schmit, K., New York State West Nile Virus Avian Surveillance Team. 2001a. Dead bird surveillance as an early warning system for West Nile virus. Emerging Infectious Diseases 7:631-635.

Eidson, M., Miller, J., Kramer, L., Cherry, B., Hagiwara, Y., West Nile Virus Bird Mortality Analysis Group. 2001b. Dead crow densities and human cases of West Nile virus, New York State, 2000. Emerging Infectious Diseases 7:662-664.

Engler, O., Savini, G., Papa, A., Figuerola, J., Groschup, M.H., Kampen, H., Medlock, J., Vaux, A., Wilson, A., Werner, D., Jöst, H., Goffredo, M., Capelli, G., Federici, V., Tonolla, M., Patocchi, N., Flacio, E., Portmann, J., Rossi-Pedruzzi, A., Mourelatos, S., Ruiz, S., Vázquez, A., Calzolari, M., Bonilauri, P., Dottori, M., Schaffner, F., Mathis, A., Johnson, N. 2013. European surveillance for West Nile virus in mosquito populations. International Journal of Environmental Research and Public Health 10:4869-4895.

Faraj, C., Elkohli, M., Lyagoubi, M. 2006. The gonotrophic cycle of Culex pipiens (Diptera: Culicidae), West Nile Virus potential vector, in Morocco: Evaluation of its duration in laboratory. Bulletin de la Societe de Pathologie Exotique 99:119-121.

Farajollahi, A., Fonseca, D.M., Kramer, L.D., Kilpatrick, A.M. 2011. "Bird biting" mosquitoes and human disease: a review of the role of Culex pipiens complex mosquitoes in epidemiology. Infection, Genetics and Evolution 11:1577-1585.

Fonseca, D.M., Keyghobadi, N., Malcolm, C.A., Mehmet, C., Schaffner, F., Mogi, M., Fleischer, R.C., Wilkerson, R.C. 2004. Emerging vectors in the Culex pipiens complex. Science 303:1535-1538.

Fortuna, C., Remoli, M.E., Di Luca, M., Severini, F., Toma, L., Benedetti, E., Bucci, P., Montarsi, F., Minelli, G., Boccolini, D., Romi, R., Ciufolini, M.G. 2015a. Experimental studies on comparison of the vector competence of four Italian Culex pipiens populations for West Nile virus. Parasites \& Vectors 8:463.

Fortuna, C., Remoli, M.E., Severini, F., Di Luca, M., Toma, L., Fois, F., Bucci, P., Boccolini, D., Romi, R., Ciufolini, M.G. 2015b. Evaluation of vector competence for West Nile virus in Italian Stegomyia albopicta (=Aedes albopictus) mosquitoes. Medical and Veterinary Entomology 29:430-433.

Fragkoudis, R., Attarzadeh-Yazdi, G., Nash, A.A., Fazakerley, J.K., Kohl, A. 2009. Advances in dissecting mosquito innate immune responses to arbovirus infection. Journal of General Virology 90:2061-2072.

Franz, A., Kantor, A., Passarelli, A., Clem, R. 2015. Tissue barriers to arbovirus infection in mosquitoes. Viruses 7:3741-3767.

Franz, A.W.E., Sanchez-Vargas, I., Adelman, Z.N., Blair, C.D., Beaty, B.J., James, A.A., Olson, K.E. 2006. Engineering RNA interference-based resistance to dengue virus type 2 in genetically modified Aedes aegypti. Proceedings of the National Academy of Sciences 103:4198-4203. 
Fritz, M.L., Walker, E.D., Miller, J.R., Severson, D.W., Dworkin, I. 2015. Divergent host preferences of above- and below-ground Culex pipiens mosquitoes and their hybrid offspring. Medical and Veterinary Entomology 29:115-123.

Fros, J.J., Geertsema, C., Vogels, C.B.F., Roosjen, P.P., Failloux, A.B., Vlak, J.M., Koenraadt, C.J.M., Takken, W., Pijlman, G.P. 2015a. West Nile Virus: High transmission rate in north-western European mosquitoes indicates its epidemic potential and warrants increased surveillance. PLoS Neglected Tropical Diseases 9:e0003956.

Fros, J.J., Miesen, P., Vogels, C.B.F., Gaibani, P., Sambri, V., Martina, B.E., Koenraadt, C.J.M., van Rij, R.P., Vlak, J.M., Takken, W., Pijlman, G.P. 2015b. Comparative Usutu and West Nile virus transmission potential by local Culex pipiens mosquitoes in northwestern Europe. One Health 1:31-36.

Gargan, T.P., Bailey, C.L., Higbee, G.A., Gad, A., El Said, S. 1983. The effect of laboratory colonization on the vector-pathogen interactions of Egyptian Culex pipiens and Rift Valley Fever Virus. The American Journal of Tropical Medicine and Hygiene 32:11541163.

Garrett-Jones, C. 1964a. The human blood index of malaria vectors in relation to epidemiological assessment. Bulletin of the World Health Organization 30:241-261.

Garrett-Jones, C. 1964b. Prognosis for interruption of malaria transmission through assessment of the mosquito's vectorial capacity. Nature 204:1173-1175.

Garrett-Jones, C. \& Shidrawi, G.R. 1969. Malaria vectorial capacity of a population of Anopheles gambiae: An exercise in epidemiological entomology. Bulletin of the World Health Organization 40:531-545.

Giberson, D.J., Dau-Schmidt, K., Dobrin, M. 2007. Mosquito species composition, phenology and distribution (Diptera: Culicidae) on Prince Edward Island. Journal of the Acadian Entomological Society 3:7-27.

Girard, Y.A., Klingler, K.A., Higgs, S. 2004. West Nile virus dissemination and tissue tropisms in orally infected Culex pipiens quinquefasciatus. Vector-Borne and zoonotic diseases 4:109-122.

Girard, Y.A., Mayhew, G.F., Fuchs, J.F., Li, H., Schneider, B.S., McGee, C.E., Rocheleau, T.A., Helmy, H., Christensen, B.M., Higgs, S. 2010. Transcriptome changes in Culex quinquefasciatus (Diptera: Culicidae) salivary glands during West Nile virus infection. Journal of Medical Entomology 47:421-435.

Girard, Y.A., Popov, V., Wen, J., Han, V., Higgs, S. 2005. Ultrastructural study of West Nile virus pathogenesis in Culex pipiens quinquefasciatus (Diptera: Culicidae). Journal of Medical Entomology 42:429-444.

Girard, Y.A., Schneider, B.S., McGee, C.E., Wen, J., Han, V.C., Popov, V., Mason, P.W., Higgs, S. 2007. Salivary gland morphology and virus transmission during long-term cytopathologic West Nile virus infection in Culex mosquitoes. The American Journal of Tropical Medicine and Hygiene 76:118-128.

Glaser, R.L. \& Meola, M.A. 2010. The native Wolbachia endosymbionts of Drosophila melanogaster and Culex quinquefasciatus increase host resistance to West Nile virus infection. PLoS One 5:e11977.

Goddard, L.B., Roth, A.E., Reisen, W.K., Scott, T.W. 2002. Vector competence of California mosquitoes for West Nile virus. Emerging infectious diseases 8:1385-1391. 
Göertz, G.P., Fros, J.J., Miesen, P., Vogels, C.B.F., van der Bent, M.L., Geertsema, C., Koenraadt, C.J.M., van Rij, R.P., van Oers, M.M., Pijlman, G.P. 2016. Non-coding subgenomic flavivirus RNA is processed by the mosquito RNAi machinery and determines West Nile virus transmission by Culex pipiens mosquitoes. Journal of Virology 90:10145-10159.

Göertz, G.P., Vogels, C.B.F., Geertsema, C., Koenraadt, C.J.M., Pijlman, G.P. 2017. Mosquito co-infection with Zika and chikungunya virus allows simultaneous transmission without affecting vector competence of Aedes aegypti. PLoS Neglected Tropical Diseases 11:e0005654.

Gomes, B., Kioulos, E., Papa, A., Almeida, A.P.G., Vontas, J., Pinto, J. 2013a. Distribution and hybridization of Culex pipiens forms in Greece during the West Nile virus outbreak of 2010. Infection, Genetics and Evolution 16:218-225.

Gomes, B., Sousa, C.A., Novo, M.T., Freitas, F.B., Alves, R., Côrte-Real, A.R., Salgueiro, P., Donnelly, M.J., Almeida, A.P., Pinto, J. 2009. Asymmetric introgression between sympatric molestus and pipiens forms of Culex pipiens (Diptera: Culicidae) in the Comporta region, Portugal. BMC Evolutionary Biology 9:262.

Gomes, B., Sousa, C.A., Vicente, J.L., Pinho, L., Calderón, I., Arez, E., Almeida, A.P.G., Donnelly, M.J., Pinto, J. 2013b. Feeding patterns of molestus and pipiens forms of Culex pipiens (Diptera: Culicidae) in a region of high hybridization. Parasites \& Vectors 6:93.

Gonçalves, D. \& Hunziker, P. 2016. Transmission-blocking strategies: the roadmap from laboratory bench to the community. Malaria Journal 15:95

Gray, S.M. \& Banerjee, N. 1999. Mechanisms of arthropod transmission of plant and animal viruses. Microbiology and Molecular Biology Reviews 63:128-148.

Grimstad, P.R., Craig Jr, G.B., Ross, Q.E., Yuill, T.M. 1977. Aedes triseriatus and La Crosse virus: geographic variation in vector susceptibility and ability to transmit. The American Journal of Tropical Medicine and Hygiene 26:990-996.

Grimstad, P.R., Ross, Q.E., Craig, G.B. 1980. Aedes triseriatus (Diptera: Culicidae) and La Crosse virus II. Modification of mosquito feeding behavior by virus infection. Journal of Medical Entomology 17:1-7.

Guy, E.C. \& Stanek, G. 1991. Detection of Borrelia burgdorferi in patients with Lyme disease by the polymerase chain reaction. Journal of Clinical Pathology 44:610-611.

Hahn, M.B., Monaghan, A.J., Hayden, M.H., Eisen, R.J., Delorey, M.J., Lindsey, N.P., Nasci, R.S., Fischer, M. 2015. Meteorological conditions associated with increased incidence of West Nile virus disease in the United States, 2004-2012. The American Journal of Tropical Medicine and Hygiene 92:1013-1022.

Hamer, G.L., Kitron, U.D., Goldberg, T.L., Brawn, J.D., Loss, S.R., Ruiz, M.O., Hayes, D.B., Walker, E.D. 2009. Host selection by Culex pipiens mosquitoes and West Nile virus amplification. The American Journal of Tropical Medicine and Hygiene 80:268-278.

Harbach, R.E. 2012. Culex pipiens: Species versus species complex - taxonomic history and perspective. Journal of the American Mosquito Control Association 28:10-23.

Hardy, J.L., Houk, E.J., Kramer, L.D., Reeves, W.C. 1983. Intrinsic factors affecting vector competence of mosquitoes for arboviruses. Annual Review of Entomology 28:229-262.

Harrison, X.A. 2014. Using observation-level random effects to model overdispersion in count data in ecology and evolution. PeerJ 2:e616. 
Hartemink, N., Cianci, D., Reiter, P. 2015. R0 for vector-borne diseases: Impact of the assumption for the duration of the extrinsic incubation period. Vector-Borne and Zoonotic Diseases 15:215-217.

Hartemink, N., Vanwambeke, S.O., Heesterbeek, H., Rogers, D., Morley, D., Pesson, B., Davies, C., Mahamdallie, S., Ready, P. 2011. Integrated mapping of establishment risk for emerging vector-borne infections: a case study of canine leishmaniasis in Southwest France. PLoS One 6:e20817.

Hartemink, N.A., Davis, S.A., Reiter, P., Hubálek, Z., Heesterbeek, J.A.P. 2007. Importance of bird-to-bird transmission for the establishment of West Nile virus. Vector-Borne and Zoonotic Diseases 7:575-584.

Hartemink, N.A., Purse, B.V., Meiswinkel, R., Brown, H.E., de Koeijer, A., Elbers, A.R.W., Boender, G.J., Rogers, D.J., Heesterbeek, J.A.P. 2009. Mapping the basic reproduction number (R0) for vector-borne diseases: A case study on bluetongue virus. Epidemics 1:153-161.

Hartemink, N.A., Randolph, S.E., Davis, S.A., Heesterbeek, J.A.P. 2008. The basic reproduction number for complex disease systems: defining RO for tick-borne infections. American Naturalist 171:743-754.

Hartley, D.M., Barker, C.M., Le Menach, A., Niu, T., Gaff, H.D., Reisen, W.K. 2012. Effects of temperature on emergence and seasonality of West Nile virus in California. The American Journal of Tropical Medicine and Hygiene 86:884-894.

Hayes, E.B. \& Gubler, D.J. 2006. West Nile Virus: Epidemiology and clinical features of an emerging epidemic in the United States. Annual Review of Medicine 57:181-194.

Hayes, E.B., Komar, N., Nasci, R.S., Montgomery, S.P., O'Leary, D.R., Campbell, G.L. 2005. Epidemiology and transmission dynamics of West Nile virus disease. Emerging Infectious Diseases 11:1167-1173.

Haylock, M.R., Hofstra, N., Klein Tank, A.M.G., Klok, E.J., Jones, P.D., New, M.C.D. 2008. A European daily high-resolution gridded data set of surface temperature and precipitation for 1950-2006. Journal of Geophysical Research: Atmospheres 113:D20119.

Hegde, S., Rasgon, J.L., Hughes, G.L. 2015. The microbiome modulates arbovirus transmission in mosquitoes. Current Opinion in Virology 15:97-102.

Hess, A.M., Prasad, A.N., Ptitsyn, A., Ebel, G.D., Olson, K.E., Barbacioru, C., Monighetti, C., Campbell, C.L. 2011. Small RNA profiling of dengue virus-mosquito interactions implicates the PIWI RNA pathway in anti-viral defense. BMC Microbiology 11:45.

Hesson, J.C., Östman, Ö., Schäfer, M., Lundström, J.O. 2011. Geographic distribution and relative abundance of the sibling vector species Culex torrentium and Culex pipiens in Sweden. Vector-Borne and Zoonotic Diseases 11:1383-1389.

Hesson, J.C., Rettich, F., Merdić, E., Vignjević, G., Östman, Ö., Schäfer, M., Schaffner, F., Foussadier, R., Besnard, G., Medlock, J., Scholte, E.J., Lundström, J.O. 2013. The arbovirus vector Culex torrentium is more prevalent than Culex pipiens in northern and central Europe. Medical and Veterinary Entomology 28:179-186.

Houk, E.J., Obie, F., Hardy, J.L. 1979. Peritrophic membrane formation and the midgut barrier to arboviral infection in the mosquito, Culex tarsalis Coquillett (Insecta, Diptera). Acta Tropica 36:39-45.

Hubalek, Z. \& Halouzka, J. 1999. West Nile fever - a reemerging mosquito-borne viral disease in Europe. Emerging Infectious Diseases 5:643-650. 
Huber, K., Jansen, S., Leggewie, M., Badusche, M., Schmidt-Chanasit, J., Becker, N., Tannich, E., Becker, S.C. 2014. Aedes japonicus japonicus (Diptera: Culicidae) from Germany have vector competence for Japan encephalitis virus but are refractory to infection with West Nile virus. Parasitology Research 113:3195-3199.

Hurd, H. 2003. Manipulation of medically important insect vectors by their parasites. Annual Review of Entomology 48:141-161.

Hussain, M., Lu, G., Torres, S., Edmonds, J.H., Kay, B.H., Khromykh, A.A., Asgari, S. 2013. Effect of Wolbachia on replication of West Nile virus in a mosquito cell line and adult mosquitoes. Journal of Virology 87:851-858.

Ibañez-Justicia, A., Stroo, A., Dik, M., Beeuwkes, J., Scholte, E.J. 2015. National mosquito (Diptera: Culicidae) survey in the Netherlands 2010-2013. Journal of Medical Entomology 52:185-198.

Ingwell, L.L., Eigenbrode, S.D., Bosque-Pérez, N.A. 2012. Plant viruses alter insect behavior to enhance their spread. Scientific reports $2: 578$.

Johnson, K.N. 2015. The impact of Wolbachia on virus infection in mosquitoes. Viruses 7:5705-5717.

Jones, R.H. \& Foster, N.M. 1978. Relevance of laboratory colonies of the vector in arbovirus research - Culicoides variipennis and Bluetongue. The American Journal of Tropical Medicine and Hygiene 27:168-177.

Jonzén, N., Lindén, A., Ergon, T., Knudsen, E., Vik, J.O., Rubolini, D., Piacentini, D., Brinch, C., Spina, F., Karlsson, L., Stervander, M., Andersson, A., Waldenström, J., Lehikoinen, A., Edvardsen, E., Solvang, R., Stenseth, N.C. 2006. Rapid advance of spring arrival dates in long-distance migratory birds. Science 312:1959-1961.

Jourdain, E., Gauthier-Clerc, M., Bicout, D., Sabatier, P. 2007a. Bird migration routes and risk for pathogen dispersion into Western Mediterranean wetlands. Emerging Infectious Diseases 13:365-372.

Jourdain, E., Schuffenecker, I., Korimbocus, J., Reynard, S., Murri, S., Kayser, Y., GauthierClerc, M., Sabatier, P., Zeller, H.G. 2007b. West Nile virus in wild resident birds, Southern France, 2004. Vector-Borne and Zoonotic Diseases 7:448-452.

Jupatanakul, N., Sim, S., Dimopoulos, G. 2014. The insect microbiome modulates vector competence for arboviruses. Viruses 6:4294.

Kampen, H., Medlock, J.M., Vaux, A.G.C., Koenraadt, C.J.M., Van Vliet, A.J.H., Bartumeus, F., Oltra, A., Sousa, C.A., Chouin, S., Werner, D. 2015. Approaches to passive mosquito surveillance in the EU. Parasites \& Vectors 8:9.

Kato, N., Mueller, C.R., Fuchs, J.F., McElroy, K., Wessely, V., Higgs, S., Christensen, B.M. 2008. Evaluation of the function of a type I peritrophic matrix as a physical barrier for midgut epithelium invasion by mosquito-borne pathogens in Aedes aegypti. VectorBorne and Zoonotic Diseases 8:701-712.

Katoh, K., Misawa, K., Kuma, K., Miyata, T. 2002. MAFFT: a novel method for rapid multiple sequence alignment based on fast Fourier transform. Nucleic Acids Research 30:30593066 .

Kenney, J. \& Brault, A. 2014. The role of environmental, virological and vector interactions in dictating biological transmission of arthropod-borne viruses by mosquitoes. p. 39-83. In: Maramorosch, K. \& Murphy, F.A. Advances in Virus Research. Academic Press, Waltham. 
Kent, R., Juliusson, L., Weissmann, M., Evans, S., Komar, N. 2009. Seasonal blood-feeding behavior of Culex tarsalis (Diptera: Culicidae) in Weld County, Colorado, 2007. Journal of Medical Entomology 46:380-390.

Kenward, M.G. \& Roger, J.H. 1997. Small sample inference for fixed effects from restricted maximum likelihood. Biometrics 53:983-997.

Kilpatrick, A.M., Daszak, P., Jones, M.J., Marra, P.P., Kramer, L.D. 2006. Host heterogeneity dominates West Nile virus transmission. Proceedings of the Royal Society B: Biological Sciences 273:2327-2333.

Kilpatrick, A.M., Fonseca, D.M., Ebel, G.D., Reddy, M.R., Kramer, L.D. 2010. Spatial and temporal variation in vector competence of Culex pipiens and $C x$. restuans mosquitoes for West Nile virus. The American Journal of Tropical Medicine and Hygiene 83:607613.

Kilpatrick, A.M., Kramer, L.D., Campbell, S.R., Alleyne, E.O., Dobson, A.P., Daszak, P. 2005. West Nile virus risk assessment and the bridge vector paradigm. Emerging Infectious Diseases 11:425-429.

Kilpatrick, A.M., Meola, M.A., Moudy, R.M., Kramer, L.D. 2008. Temperature, viral genetics, and the transmission of West Nile virus by Culex pipiens mosquitoes. PLoS Pathogens 4:e1000092.

Kilpatrick, A.M. \& Randolph, S.E. 2012. Drivers, dynamics, and control of emerging vectorborne zoonotic diseases. The Lancet 380:1946-1955.

KNMI. 2014. Weerstatistieken, De Bilt. https://weerstatistieken.nl/de-bilt/2014/.

Knols, B.G.J., de Jong, R., Takken, W. 1994. Trapping system for testing olfactory responses of the malaria mosquito Anopheles gambiae in a wind tunnel. Medical and Veterinary Entomology 8:386-388.

Koella, J.C., Rieu, L., Paul, R.E.L. 2002. Stage-specific manipulation of a mosquito's hostseeking behavior by the malaria parasite Plasmodium gallinaceum. Behavioral Ecology 13:816-820.

Koenraadt, C.J.M. \& Harrington, L.C. 2008. Flushing effect of rain on container-inhabiting mosquitoes Aedes aegypti and Culex pipiens (Diptera: Culicidae). Journal of Medical Entomology 45:28-35.

Komar, N. 2003. West Nile virus: epidemiology and ecology in North America. Advances in Virus Research 61:185-234.

Komar, N., Langevin, S., Hinten, S., Nemeth, N., Edwards, E., Hettler, D., Davis, B., Bowen, R., Bunning, M. 2003. Experimental infection of North American birds with the New York 1999 strain of West Nile virus. Emerging Infectious Diseases 9:311.

Kramer, L.D., Styer, L.M., Ebel, G.D. 2008. A global perspective on the epidemiology of West Nile virus. Annual Review of Entomology 53:61-81.

Kushmaro, A., Friedlander, T.A., Levins, R. 2015. Temperature effects on the basic reproductive number (R0) of West Nile virus, based on ecological parameters: endemic vs. new emergence regions. Journal of Tropical Diseases 10:2.

Lambrechts, L., Paaijmans, K.P., Fansiri, T., Carrington, L.B., Kramer, L.D., Thomas, M.B., Scott, T.W. 2011. Impact of daily temperature fluctuations on dengue virus transmission by Aedes aegypti. Proceedings of the National Academy of Sciences 108:7460-7465. 
Lanciotti, R.S., Ebel, G.D., Deubel, V., Kerst, A.J., Murri, S., Meyer, R., Bowen, M., McKinney, N., Morrill, W.E., Crabtree, M.B., Kramer, L.D., Roehrig, J.T. 2002. Complete genome sequences and phylogenetic analysis of West Nile virus strains isolated from the United States, Europe, and the Middle East. Virology 298:96-105.

Lanciotti, R.S., Roehrig, J.T., Deubel, V., Smith, J., Parker, M., Steele, K., Crise, B., Volpe, K.E., Crabtree, M.B., Scherret, J.H., Hall, R.A., MacKenzie, J.S., Cropp, C.B., Panigrahy, B., Ostlund, E., Schmitt, B., Malkinson, M., Banet, C., Weissman, J., Komar, N., Savage, H.M., Stone, W., McNamara, T., Gubler, D.J. 1999. Origin of the West Nile Virus responsible for an outbreak of encephalitis in the northeastern United States. Science 286:2333-2337.

Lefèvre, T., Koella, J.C., Renaud, F., Hurd, H., Biron, D.G., Thomas, F. 2006. New prospects for research on manipulation of insect vectors by pathogens. PLoS Pathogens 2:e72.

Lefèvre, T. \& Thomas, F. 2008. Behind the scene, something else is pulling the strings: emphasizing parasitic manipulation in vector-borne diseases. Infection, Genetics and Evolution 8:504-519.

Leggewie, M., Badusche, M., Rudolf, M., Jansen, S., Börstler, J., Krumkamp, R., Huber, K., Krüger, A., Schmidt-Chanasit, J., Tannich, E., Becker, S.C. 2016. Culex pipiens and Culex torrentium populations from Central Europe are susceptible to West Nile virus infection. One Health 2:88-94.

Lerdthusnee, K., Romoser, W.S., Faran, M.E., Dohm, D.J. 1995. Rift Valley Fever Virus in the cardia of Culex pipiens: An immunocytochemical and ultrastructural study. The American Journal of Tropical Medicine and Hygiene 53:331-337.

Lim, S.M., Brault, A.C., van Amerongen, G., Bosco-Lauth, A.M., Romo, H., Sewbalaksing, V.D., Bowen, R.A., Osterhaus, A.D.M.E., Koraka, P., Martina, B.E.E. 2015. Susceptibility of carrion crows to experimental infection with lineage 1 and 2 West Nile Viruses. Emerging Infectious Diseases 21:1357-1365.

Lim, S.M., Brault, A.C., van Amerongen, G., Sewbalaksing, V.D., Osterhaus, A.D.M.E., Martina, B.E.E., Koraka, P. 2014. Susceptibility of European jackdaws (Corvus monedula) to experimental infection with lineage 1 and 2 West Nile viruses. Journal of General Virology 95:1320-1329.

Lima-Camara, T.N., Bruno, R.V., Luz, P.M., Castro, M.G., Lourenço-de-Oliveira, R., Sorgine, M.H.F., Peixoto, A.A. 2011. Dengue infection increases the locomotor activity of Aedes aegypti females. PLoS One 6:e17690.

Linke, S., Niedrig, M., Kaiser, A., Ellerbrok, H., Müller, K., Müller, T., Conraths, F.J., Mühle, R.-U., Schmidt, D., Köppen, U., Bairlein, F., Berthold, P., Pauli, G. 2007. Serologic evidence of West Nile virus infections in wild birds captured in Germany. The American Journal of Tropical Medicine and Hygiene 77:358-364.

Liu, B., Behura, S.K., Clem, R.J., Schneemann, A., Becnel, J., Severson, D.W., Zhou, L. 2013. P53-mediated rapid induction of apoptosis conveys resistance to viral infection in Drosophila melanogaster. PLoS Pathogens 9:e1003137.

Lorenz, L., Beaty, B.J., Aitken, T.H.G., Wallis, G.P., Tabachnick, W.J. 1984. The effect of colonization upon Aedes aegypti susceptibility to oral infection with Yellow Fever virus. The American Journal of Tropical Medicine and Hygiene 33:690-694.

Luplertlop, N., Surasombatpattana, P., Patramool, S., Dumas, E., Wasinpiyamongkol, L., Saune, L., Hamel, R., Bernard, E., Sereno, D., Thomas, F. 2011. Induction of a peptide with activity against a broad spectrum of pathogens in the Aedes aegypti salivary gland, following infection with dengue virus. PLoS Pathogens 7:e1001252. 
Macdonald, G. 1952. The analysis of equilibrium in malaria. Tropical Diseases Bulletin 49:813829.

Macdonald, G. 1957. The epidemiology and control of malaria. Oxford University Press, London.

Madder, D.J., Surgeoner, G.A., Helson, B.V. 1983. Number of generations, egg production, and developmental time of Culex pipiens and Culex restuans (Diptera: Culicidae) in Southern Ontario Journal of Medical Entomology 20:275-287.

Marcantonio, M., Rizzoli, A., Metz, M., Rosà, R., Marini, G., Chadwick, E., Neteler, M. 2015. Identifying the environmental conditions favouring West Nile virus outbreaks in Europe. PLoS One 10:e0121158.

Marshall, J.F. \& Staley, J. 1935. Autogenous strains of Culex pipiens (Diptera, Culicidae). Nature 136:641.

Matser, A., Hartemink, N., Heesterbeek, H., Galvani, A., Davis, S. 2009. Elasticity analysis in epidemiology: an application to tick-borne infections. Ecology Letters 12:1298-1305.

Mattingly, P.F., Rozeboom, L.E., Knight, K.L., Laven, H., Drummond, F.H., Christophers, S.R., Shute, P.G. 1951. The Culex pipiens complex. Transactions of the Royal Entomological Society of London 102:331-342.

McGee, C.E., Shustov, A.V., Tsetsarkin, K., Frolov, I.V., Mason, P.W., Vanlandingham, D.L., Higgs, S. 2010. Infection, dissemination, and transmission of a West Nile virus green fluorescent protein infectious clone by Culex pipiens quinquefasciatus mosquitoes. Vector-Borne and Zoonotic Diseases 10:267-274.

Medzhitov, R., Schneider, D.S., Soares, M.P. 2012. Disease tolerance as a defense strategy. Science 335:936-941.

Meehl, G.A. \& Tebaldi, C. 2004. More intense, more frequent, and longer lasting heat waves in the 21st century. Science 305:994-997.

Mendoza, M.Y., Salas-Benito, J.S., Lanz-Mendoza, H., Hernández-Martínez, S., Del Angel, R.M. 2002. A putative receptor for dengue virus in mosquito tissues: localization of a 45-kDa glycoprotein. The American Journal of Tropical Medicine and Hygiene 67:7684.

Mesterton-Gibbons, M. 1993. Why demographic elasticities sum to one: A postscript to de Kroon et al. Ecology 74:2467-2468.

Micieli, M.V. \& Glaser, R.L. 2014. Somatic Wolbachia (Rickettsiales: Rickettsiaceae) levels in Culex quinquefasciatus and Culex pipiens (Diptera: Culicidae) and resistance to West Nile virus infection. Journal of Medical Entomology 51:189-199.

Morens, D.M., Folkers, G.K., Fauci, A.S. 2004. The challenge of emerging and re-emerging infectious diseases. Nature 430:242-249.

Morse, S.S. 1995. Factors in the emergence of infectious diseases. Emerging Infectious Diseases 1:7-15.

Mostashari, F., Bunning, M.L., Kitsutani, P.T., Singer, D.A., Nash, D., Cooper, M.J., Katz, N., Liljebjelke, K.A., Biggerstaff, B.J., Fine, A.D., Layton, M.C., Mullin, S.M., Johnson, A.J., Martin, D.A., Hayes, E.B., Campbell, G.L. 2001. Epidemic West Nile encephalitis, New York, 1999: results of a household-based seroepidemiological survey. The Lancet 358:261-264.

Mourya, D.T., Yadav, P., Mishra, A.C. 2004. Effect of temperature stress on immature stages and susceptibility of Aedes aegypti mosquitoes to Chikungunya virus. The American Journal of Tropical Medicine and Hygiene 70:346-350. 
Muñoz, J., Ruiz, S., Soriguer, R., Alcaide, M., Viana, D.S., Roiz, D., Vázquez, A., Figuerola, J. 2012. Feeding patterns of potential West Nile virus vectors in South-West Spain. PLoS One 7:e39549.

Murdock, C.C., Paaijmans, K.P., Cox-Foster, D., Read, A.F., Thomas, M.B. 2012. Rethinking vector immunology: the role of environmental temperature in shaping resistance. Nature Reviews Microbiology 10:869-876.

Murgue, B., Murri, S., Triki, H., Deubel, V., Zeller, H.G. 2001. West Nile in the Mediterranean basin: 1950-2000. Annals of the New York Academy of Sciences 951:117-126.

Nelms, B.M., Kothera, L., Thiemann, T., Macedo, P.A., Savage, H.M., Reisen, W.K. 2013. Phenotypic variation among Culex pipiens complex (Diptera: Culicidae) populations from the Sacramento Valley, California: horizontal and vertical transmission of West Nile virus, diapause potential, autogeny, and host selection. The American Journal of Tropical Medicine and Hygiene 89:1168-1178.

Nguyen, T.H., Nguyen, H.L., Nguyen, T.Y., Vu, S.N., Tran, N.D., Le, T.N., Vien, Q.M., Bui, T.C., Le, H.T., Kutcher, S., Hurst, T.P., Duong, T.T.H., Jeffery, J.A.L., Darbro, J.M., Kay, B.H., Iturbe-Ormaetxe, I., Popovici, J., Montgomery, B.L., Turley, A.P., Zigterman, F., Cook, H., Cook, P.E., Johnson, P.H., Ryan, P.A., Paton, C.J., Ritchie, S.A., Simmons, C.P., O'Neill, S.L., Hoffmann, A.A. 2015. Field evaluation of the establishment potential of wmelpop Wolbachia in Australia and Vietnam for dengue control. Parasites \& Vectors 8:563.

O'Neill, S.L., Hoffmann, A.A., Werren, J.H. 1997. Influential passengers: inherited microorganisms and arthropod reproduction. Oxford University Press, Oxford

Osório, H.C., Zé-Zé, L., Alves, M.J. 2012. Host-feeding patterns of Culex pipiens and other potential mosquito vectors (Diptera: Culicidae) of West Nile virus (Flaviviridae) collected in Portugal. Journal of Medical Entomology 49:717-721.

Osório, H.C., Zé-Zé, L., Amaro, F., Nunes, A., Alves, M.J. 2014. Sympatric occurrence of Culex pipiens (Diptera, Culicidae) biotypes pipiens, molestus and their hybrids in Portugal, Western Europe: feeding patterns and habitat determinants. Medical and Veterinary Entomology 28:103-109.

Papa, A., Xanthopoulou, K., Gewehr, S., Mourelatos, S. 2011. Detection of West Nile virus lineage 2 in mosquitoes during a human outbreak in Greece. Clinical Microbiology and Infection 17:1176-1180.

Papa, A., Xanthopoulou, K., Tsioka, A., Kalaitzopoulou, S., Mourelatos, S. 2013. West Nile virus in mosquitoes in Greece. Parasitology Research 112:1551-1555.

Paradkar, P.N., Trinidad, L., Voysey, R., Duchemin, J.-B., Walker, P.J. 2012. Secreted Vago restricts West Nile virus infection in Culex mosquito cells by activating the Jak-STAT pathway. Proceedings of the National Academy of Sciences 109:18915-18920.

Pates, H.V., Takken, W., Stuke, K., Curtis, C.F. 2001. Differential behaviour of Anopheles gambiae sensu stricto (Diptera: Culicidae) to human and cow odours in the laboratory. Bulletin of Entomological Research 91:289-296.

Paz, S. 2015. Climate change impacts on West Nile virus transmission in a global context. Philosophical Transactions of the Royal Society B: Biological Sciences 370:20130561.

Paz, S., Malkinson, D., Green, M.S., Tsioni, G., Papa, A., Danis, K., Sirbu, A., Ceianu, C., Katalin, K., Ferenczi, E., Zeller, H., Semenza, J.C. 2013. Permissive summer temperatures of the 2010 European West Nile fever upsurge. PLoS One 8:e56398. 
Paz, S. \& Semenza, J. 2013. Environmental drivers of West Nile fever epidemiology in Europe and Western Asia - a review. International Journal of Environmental Research and Public Health 10:3543.

Pesko, K.N. \& Ebel, G.D. 2012. West Nile virus population genetics and evolution. Infection, Genetics and Evolution 12:181-190.

Petersen, L.R., Brault, A.C., Nasci, R.S. 2013. West Nile virus: review of the literature. JAMA 310:308-315.

Platonov, A.E., Fedorova, M.V., Karan, L.S., Shopenskaya, T.A., Platonova, O.V., Zhuravlev, V.I. 2008. Epidemiology of West Nile infection in Volgograd, Russia, in relation to climate change and mosquito (Diptera: Culicidae) bionomics. Parasitology Research 103:45-53.

Prasad, A.N., Brackney, D., Ebel, G. 2013. The role of innate immunity in conditioning mosquito susceptibility to West Nile virus. Viruses 5:3142.

Puri, S.N., Mendki, M.J., Sukumaran, D., Ganesan, K., Prakash, S., Sekhar, K. 2006. Electroantennogram and behavioral responses of Culex quinquefasciatus (Diptera: Culicidae) females to chemicals found in human skin emanations. Journal of Medical Entomology 43:207-213.

Qiu, Y.-T., Gort, G., Torricelli, R., Takken, W., van Loon, J.J.A. 2013. Effects of blood-feeding on olfactory sensitivity of the malaria mosquito Anopheles gambiae: Application of mixed linear models to account for repeated measurements. Journal of Insect Physiology 59:1111-1118.

R Core Team. 2016. R: A language and environment for statistical computing.

Ramirez, J.L. \& Dimopoulos, G. 2010. The Toll immune signaling pathway control conserved anti-dengue defenses across diverse $A e$. aegypti strains and against multiple dengue virus serotypes. Developmental \& Comparative Immunology 34:625-629.

Reisen, W.K., Fang, Y., Martinez, V.M. 2006. Effects of temperature on the transmission of West Nile virus by Culex tarsalis (Diptera: Culicidae). Journal of Medical Entomology 43:309-317.

Reisen, W.K., Meyer, R.P., Presser, S.B., Hardy, J.L. 1993. Effect of temperature on the transmission of Western equine encephalomyelitis and St. Louis encephalitis viruses by Culex tarsalis (Diptera: Culicidae). Journal of Medical Entomology 30:151-160.

Reusken, C.B.E.M., de Vries, A., Buijs, J., Braks, M.A.H., den Hartog, W., Scholte, E.J. 2010a. First evidence for presence of Culex pipiens biotype molestus in the Netherlands, and of hybrid biotype pipiens and molestus in northern Europe. Journal of Vector Ecology 35:210-212.

Reusken, C.B.E.M., De Vries, A., Den Hartog, W., Braks, M.A.H., Scholte, E.J. 2010b. A study of the circulation of West Nile virus in mosquitoes in a potential high-risk area for arbovirus circulation in the Netherlands, "De Oostvaardersplassen". European Mosquito Bulletin 28:69-83.

Rijks, J.M., Kik, M.L., Slaterus, R., Foppen, R.P.B., Stroo, A., ljzer, J., Stahl, J., Gröne, A., Koopmans, M.G.P., van der Jeugd, H.P., Reusken, C.B.E.M. 2016. Widespread Usutu virus outbreak in birds in the Netherlands, 2016. Eurosurveillance 21:30391.

Rizzoli, A., Bolzoni, L., Chadwick, E.A., Capelli, G., Montarsi, F., Grisenti, M., de la Puente, J.M., Muñoz, J., Figuerola, J., Soriguer, R., Anfora, G., Di Luca, M., Rosà, R. 2015a. Understanding West Nile virus ecology in Europe: Culex pipiens host feeding preference in a hotspot of virus emergence. Parasites \& Vectors 8:213. 
Rizzoli, A., Jiménez-Clavero, M.A., Barzon, L., Cordioli, P., Figuerola, J., Koraka, P., Martina, B., Moreno, A., Nowotny, N., Pardigon, N., Sanders, N., Ulbert, S., Tenorio, A. 2015b. The challenge of West Nile virus in Europe: Knowledge gaps and research priorities. Eurosurveillance 20:21135.

Rockx, B., Asten, L.V., Wijngaard, C.V.D., Godeke, G.-J., Goehring, L., Vennema, H., Avoort, H.V.D., Pelt, W.V., Koopmans, M. 2006. Syndromic surveillance in The Netherlands for the early detection of West Nile virus epidemics. Vector-Borne and Zoonotic Diseases $6: 161-169$.

Romoser, W.S., Turell, M.J., Lerdthusnee, K., Neira, M., Dohm, D., Ludwig, G., Wasieloski, L. 2005. Pathogenesis of Rift Valley fever virus in mosquitoes - tracheal conduits \& the basal lamina as an extra-cellular barrier. Archives of Virology 19:89-100.

Romoser, W.S., Wasieloski, L.P., Pushko, P., Kondig, J.P., Lerdthusnee, K., Neira, M., Ludwig, G.V. 2004. Evidence for arbovirus dissemination conduits from the mosquito (Diptera: Culicidae) midgut. Journal of Medical Entomology 41:467-475.

Rossignol, P.A., Ribeiro, J.M.C., Spielman, A. 1986. Increased biting rate and reduced fertility in sporozoite-infected mosquitoes. The American Journal of Tropical Medicine and Hygiene 35:277-279.

Rudolf, M., Czajka, C., Börstler, J., Melaun, C., Jöst, H., von Thien, H., Badusche, M., Becker, N., Schmidt-Chanasit, J., Krüger, A., Tannich, E., Becker, S. 2013. First nationwide surveillance of Culex pipiens complex and Culex torrentium mosquitoes demonstrated the presence of Culex pipiens biotype pipiens/molestus hybrids in Germany. PLoS One 8:e71832.

Ruybal, J.E., Kramer, L.D., Kilpatrick, A.M. 2016. Geographic variation in the response of Culex pipiens life history traits to temperature. Parasites \& Vectors 9:116.

Salas-Benito, J.S. \& De Nova-Ocampo, M. 2015. Viral interference and persistence in mosquito-borne flaviviruses. Journal of Immunology Research 2015:14.

Salazar, M.I., Richardson, J.H., Sánchez-Vargas, I., Olson, K.E., Beaty, B.J. 2007. Dengue virus type 2: replication and tropisms in orally infected Aedes aegypti mosquitoes. BMC Microbiology 7:1.

Sambri, V., Capobianchi, M., Charrel, R., Fyodorova, M., Gaibani, P., Gould, E., Niedrig, M., Papa, A., Pierro, A., Rossini, G., Varani, S., Vocale, C., Landini, M.P. 2013. West Nile virus in Europe: emergence, epidemiology, diagnosis, treatment, and prevention. Clinical Microbiology and Infection 19:699-704.

Sanburg, L.L. \& Larsen, J.R. 1973. Effect of photoperiod and temperature on ovarian development in Culex pipiens pipiens. Journal of Insect Physiology 19:1173-1190.

Sánchez-Vargas, I., Scott, J.C., Poole-Smith, B.K., Franz, A.W.E., Barbosa-Solomieu, V., Wilusz, J., Olson, K.E., Blair, C.D. 2009. Dengue virus type 2 infections of Aedes aegypti are modulated by the mosquito's RNA interference pathway. PLoS Pathogens 5:e1000299.

Saraiva, R.G., Kang, S., Simões, M.L., Angleró-Rodríguez, Y.I., Dimopoulos, G. 2016. Mosquito gut antiparasitic and antiviral immunity. Developmental \& Comparative Immunology 64:53-64.

Sardelis, M.R. \& Turell, M.J. 2001. Ochlerotatus j. japonicus in Frederick County, Maryland: discovery, distribution, and vector competence for West Nile virus. Journal of the American Mosquito Control Association 17:137-141. 
Sardelis, M.R., Turell, M.J., Dohm, D.J., O'Guinn, M.L. 2001. Vector competence of selected North American Culex and Coquillettidia mosquitoes for West Nile virus. Emerging Infectious Diseases 7:1018.

Schäfer, M. \& Lundström, J.O. 2001. Comparison of mosquito (Diptera: Culicidae) fauna characteristics of forested wetlands in Sweden. Annals of the Entomological Society of America 94:576-582.

Schaffner, F., Angel, G., Geoffrey, B., Hervy, J.P., Rhaiem, A., Brunhes, J. 2001. CD-ROM The mosquitoes of Europe an identification and training program.

Scholle, F., Girard, Y.A., Zhao, Q., Higgs, S., Mason, P.W. 2004. trans-Packaged West Nile virus-like particles: infectious properties in vitro and in infected mosquito vectors. Journal of Virology 78:11605-11614.

Semenza, J.C., Tran, A., Espinosa, L., Sudre, B., Domanovic, D., Paz, S. 2016. Climate change projections of West Nile virus infections in Europe: implications for blood safety practices. Environmental Health 15:S28.

Shaman, J. \& Day, J.F. 2007. Reproductive phase locking of mosquito populations in response to rainfall frequency. PLoS One 2:e331.

Shaman, J., Day, J.F., Stieglitz, M. 2005. Drought-induced amplification and epidemic transmission of West Nile virus in Southern Florida. Journal of Medical Entomology 42:134-141.

Shute, P.G. 1941. A species of mosquito infesting deep shelters in London. The Lancet 237:67.

Sim, C. \& Denlinger, D.L. 2008. Insulin signaling and FOXO regulate the overwintering diapause of the mosquito Culex pipiens. Proceedings of the National Academy of Sciences 105:6777-6781.

Sim, S., Jupatanakul, N., Dimopoulos, G. 2014. Mosquito immunity against arboviruses. Viruses 6:4479-4504.

Sim, S., Jupatanakul, N., Ramirez, J.L., Kang, S., Romero-Vivas, C.M., Mohammed, H., Dimopoulos, G. 2013. Transcriptomic profiling of diverse Aedes aegypti strains reveals increased basal-level immune activation in dengue virus-refractory populations and identifies novel virus-vector molecular interactions. PLoS Neglected Tropical Diseases 7:e2295.

Sim, S., Ramirez, J.L., Dimopoulos, G. 2012. Dengue virus infection of the Aedes aegypti salivary gland and chemosensory apparatus induces genes that modulate infection and blood-feeding behavior. PLoS Pathogens 8:e1002631.

Simpson, J.E., Folsom-O'Keefe, C.M., Childs, J.E., Simons, L.E., Andreadis, T.G., DiukWasser, M.A. 2009. Avian host-selection by Culex pipiens in experimental trials. PLoS One 4:e7861.

Smallegange, R.C., Schmied, W.H., van Roey, K.J., Verhulst, N.O., Spitzen, J., Mukabana, W.R., Takken, W. 2010. Sugar-fermenting yeast as an organic source of carbon dioxide to attract the malaria mosquito Anopheles gambiae. Malaria Journal 9:292.

Smallegange, R.C., van Gemert, G.-J., van de Vegte-Bolmer, M., Gezan, S., Takken, W., Sauerwein, R.W., Logan, J.G. 2013. Malaria infected mosquitoes express enhanced attraction to human odor. PLoS One 8:e63602.

Smith, D.L., Battle, K.E., Hay, S.I., Barker, C.M., Scott, T.W., McKenzie, F.E. 2012. Ross, Macdonald, and a theory for the dynamics and control of mosquito-transmitted pathogens. PLoS Pathogens 8:e1002588. 
Smith, J.L. \& Fonseca, D.M. 2004. Rapid assays for identification of members of the Culex (Culex) pipiens complex, their hybrids, and other sibling species (Diptera: Culicidae). The American Journal of Tropical Medicine and Hygiene 70:339-345.

Smithburn, K.C., Hughes, T.P., Burke, A.W., Paul, J.H. 1940. A neurotropic virus isolated from the blood of a native of Uganda. The American Journal of Tropical Medicine and Hygiene 20:471-492.

Snapinn, K.W., Holmes, E.C., Young, D.S., Bernard, K.A., Kramer, L.D., Ebel, G.D. 2007. Declining growth rate of West Nile virus in North America. Journal of Virology 81:25312534.

Snow, K.R. 1990. Naturalists' Handbooks 14: Mosquitoes. Richmond Publishing Co., Slough.

Souza-Neto, J.A., Sim, S., Dimopoulos, G. 2009. An evolutionary conserved function of the JAK-STAT pathway in anti-dengue defense. Proceedings of the National Academy of Sciences 106:17841-17846.

Spielman, A. 2001. Structure and seasonality of nearctic Culex pipiens populations. Annals of the New York Academy of Sciences 951:220-234.

Styer, L.M., Meola, M.A., Kramer, L.D. 2007. West Nile virus infection decreases fecundity of Culex tarsalis females. Journal of Medical Entomology 44:1074-1085.

Syed, Z. \& Leal, W.S. 2009. Acute olfactory response of Culex mosquitoes to a human- and bird-derived attractant. Proceedings of the National Academy of Sciences 106:1880318808.

Takken, W. 1991. The role of olfaction in host-seeking of mosquitoes: a review. International Journal of Tropical Insect Science 12:287-295.

Targett, G.A.T. 2006. Parasites, arthropod vectors, and immune responses. Parasite Immunology 28:117-119.

Thomas, R.E., Wu, W.-K., Verleye, D., Rai, K.S. 1993. Midgut basal lamina thickness and dengue-1 virus dissemination rates in laboratory strains of Aedes albopictus (Diptera: Culicidae). Journal of Medical Entomology 30:326-331.

Tomasello, D. \& Schlagenhauf, P. 2013. Chikungunya and dengue autochthonous cases in Europe, 2007-2012. Travel Medicine and Infectious Disease 11:274-284.

Turell, M.J., Dohm, D.J., Sardelis, M.R., O'guinn, M.L., Andreadis, T.G., Blow, J.A. 2005. An update on the potential of North American mosquitoes (Diptera: Culicidae) to transmit West Nile virus. Journal of Medical Entomology 42:57-62.

Turell, M.J., Mores, C.N., Dohm, D.J., Komilov, N., Paragas, J., Lee, J.S., Shermuhemedova, D., Endy, T.P., Kodirov, A., Khodjaev, S. 2006. Laboratory transmission of Japanese encephalitis and West Nile viruses by molestus form of Culex pipiens (Diptera: Culicidae) collected in Uzbekistan in 2004. Journal of Medical Entomology 43:296-300.

Turell, M.J., O'Guinn, M.L., Dohm, D.J., Jones, J.W. 2001a. Vector competence of North American mosquitoes (Diptera: Culicidae) for West Nile virus. Journal of Medical Entomology 38:130-134.

Turell, M.J., Sardelis, M.R., Dohm, D.J., O'Guinn, M.L. 2001b. Potential North American vectors of West Nile virus. Annals of the New York Academy of Sciences 951:317-324.

Vaidyanathan, R. \& Scott, T.W. 2006. Apoptosis in mosquito midgut epithelia associated with West Nile virus infection. Apoptosis 11:1643-1651. 
Vaidyanathan, R. \& Scott, T.W. 2007. Geographic variation in vector competence for West Nile virus in the Culex pipiens (Diptera: Culicidae) complex in California. Vector-Borne and Zoonotic Diseases 7:193-198.

Valiakos, G., Touloudi, A., lacovakis, C., Athanasiou, L., Birtsas, P., Spyrou, V., Billinis, C. 2011. Molecular detection and phylogenetic analysis of West Nile virus lineage 2 in sedentary wild birds (Eurasian magpie), Greece, 2010. Eurosurveillance 16:19862.

van Houte, S., Ros, V.I.D., van Oers, M.M. 2013. Walking with insects: molecular mechanisms behind parasitic manipulation of host behaviour. Molecular Ecology 22:3458-3475.

Vazeille, M., Yébakima, A., Lourenço-de-Oliveira, R., Andriamahefazafy, B., Correira, A., Rodrigues, J.M., Veiga, A., Moreira, A., Leparc-Goffart, I., Grandadam, M. 2013. Oral receptivity of Aedes aegypti from Cape Verde for yellow fever, dengue, and chikungunya viruses. Vector-Borne and Zoonotic Diseases 13:37-40.

Vega-Almeida, T.O., Salas-Benito, M., De Nova-Ocampo, M.A., Del Angel, R.M., SalasBenito, J.S. 2013. Surface proteins of C6/36 cells involved in dengue virus 4 binding and entry. Archives of virology 158:1189-1207.

Verdonschot, P. 2002. Family Culicidae. p. 98-100. In: Beuk, P.L.T. Checklist of the Diptera in the Netherlands. KNNV Publishers, Utrecht.

Vinogradova, E.B. 2000. Culex pipiens pipiens mosquitoes: taxonomy, distribution, ecology, physiology, genetic, applied importance and control. Pensoft Publishers, Sofia.

Vinogradova, E.B. \& Shaikevich, E.V. 2007. Morphometric, physiological and molecular characteristics of underground populations of the urban mosquito Culex pipiens Linnaeus f. molestus Forskål (Diptera: Culicidae) from several areas of Russia. European Mosquito Bulletin 22:17-24.

Vogels, C.B.F., Fros, J.J., Göertz, G.P., Pijlman, G.P., Koenraadt, C.J.M. 2016a. Vector competence of northern European Culex pipiens biotypes and hybrids for West Nile virus is differentially affected by temperature. Parasites \& Vectors 9:393. (Chapter 4 of this thesis)

Vogels, C.B.F., Göertz, G.G., Pijlman, G.P., Koenraadt, C.J.M. 2017. Vector competence of northern and southern European Culex pipiens pipiens mosquitoes for West Nile virus across a gradient of temperatures. Medical and Veterinary Entomology (in press). (Chapter 5 of this thesis)

Vogels, C.B.F., Möhlmann, T.W.R., Melsen, D., Favia, G., Wennergren, U., Koenraadt, C.J.M. 2016b. Latitudinal diversity of Culex pipiens biotypes and hybrids in farm, peri-urban, and wetland habitats in Europe. PLoS One 11:e0166959. (Chapter 3 of this thesis)

Vogels, C.B.F., Van De Peppel, L.J.J., Van Vliet, A.J.H., Westenberg, M., Ibañez-Justicia, A., Stroo, A., Buijs, J.A., Visser, T.M., Koenraadt, C.J.M. 2015. Winter activity and aboveground hybridization between the two biotypes of the West Nile virus vector Culex pipiens. Vector-Borne and Zoonotic Diseases 15:619-626. (Chapter 2 of this thesis)

Wang, S. \& Jacobs-Lorena, M. 2013. Genetic approaches to interfere with malaria transmission by vector mosquitoes. Trends in Biotechnology 31:185-193.

Weaver, S.C. 2006. Evolutionary influences in arboviral disease. p. 285-314. In: Domingo, E. Quasispecies: Concept and implications for virology. Springer, Heidelberg.

Weaver, S.C. \& Barrett, A.D.T. 2004. Transmission cycles, host range, evolution and emergence of arboviral disease. Nature Reviews Microbiology 2:789-801.

Weaver, S.C. \& Reisen, W.K. 2010. Present and future arboviral threats. Antiviral Research 85:328-345. 
Weaver, S.C., Scott, T.W., Lorenz, L.H., Repik, P.M. 1991. Detection of Eastern equine encephalomyelitis virus deposition in Culiseta melanura following ingestion of radiolabeled virus in blood meals. The American Journal of Tropical Medicine and Hygiene 44:250-259.

Weitzel, T., Braun, K., Collado, A., Jöst, A., Becker, N. 2011. Distribution and frequency of Culex pipiens and Culex torrentium (Culicidae) in Europe and diagnostic allozyme markers. European Mosquito Bulletin 29:22-37.

Weitzel, T., Collado, A., Jöst, A., Pietsch, K., Storch, V., Becker, N. 2009. Genetic differentiation of populations within the Culex pipiens complex and phylogeny of related species. Journal of the American Mosquito Control Association 25:6-17.

Wheeler, S.S., Barker, C.M., Fang, Y., Veronica Armijos, M., Carroll, B.D., Husted, S., Johnson, W.O., Reisen, W.K. 2009. Differential impact of West Nile virus on California birds. The Condor 111:1-20.

Whitfield, S.G., Murphy, F.A., Sudia, W.D. 1973. St. Louis encephalitis virus: an ultrastructural study of infection in a mosquito vector. Virology 56:70-87.

Willcox, W. 1941. Mosquitoes infesting deep shelters. The Lancet 237:127.

Work, T.H., Hurlbut, H.S., Taylor, R.M. 1955. Indigenous wild birds of the Nile delta as potential West Nile virus circulating reservoirs. The American Journal of Tropical Medicine and Hygiene 4:872-888.

Xi, Z., Ramirez, J.L., Dimopoulos, G. 2008. The Aedes aegypti Toll pathway controls dengue virus infection. PLoS Pathogens 4:e1000098.

Zeller, H.G. \& Schuffenecker, I. 2004. West Nile virus: an overview of its spread in Europe and the Mediterranean Basin in contrast to its spread in the Americas. European Journal of Clinical Microbiology and Infectious Diseases 23:147-156.

Zittra, C., Flechl, E., Kothmayer, M., Vitecek, S., Rossiter, H., Zechmeister, T., Fuehrer, H.P. 2016. Ecological characterization and molecular differentiation of Culex pipiens complex taxa and Culex torrentium in eastern Austria. Parasites \& Vectors 9:197.

Zouache, K., Fontaine, A., Vega-Rua, A., Mousson, L., Thiberge, J.-M., Lourenco-DeOliveira, R., Caro, V., Lambrechts, L., Failloux, A.-B. 2014. Three-way interactions between mosquito population, viral strain and temperature underlying chikungunya virus transmission potential. Proceedings of the Royal Society B: Biological Sciences 281:20141078. 

Summary 
West Nile virus (WNV) is an arthropod-borne (arbo)virus which is causing repeated outbreaks in humans in southern and central European countries. In contrast, no human cases have been reported in northern European countries. WNV is maintained in a natural transmission cycle between mosquito vectors and bird hosts. Humans and equines may also get infected with WNV, which can result in disease, but the virus does not reach sufficient levels in the blood for further transmission to mosquitoes. Mosquitoes play a central role in the WNV transmission cycle, due to their feeding behaviour, vector competence, and dependence on environmental factors such as temperature. Mosquito-related factors may, therefore, explain the absence of WNV outbreaks in northern Europe. The aim of this thesis was to investigate the role of Culex $(C x$.$) pipiens mosquitoes in transmission of WNV in Europe, in order to$ understand differences in WNV circulation between northern and southern Europe. This thesis focuses on mosquitoes as key players in the transmission of WNV, by investigating the interaction between mosquito, virus, and temperature.

One of the main vectors of WNV is the mosquito species $C x$. pipiens, which consists of two behaviourally different biotypes, named pipiens and molestus. Mating between both biotypes results in hybrids with ideal behavioural characteristics to bridge WNV from birds to humans, due to their opportunistic feeding behaviour on both birds and mammals. To gain insight in the spatial distribution and biting activity of the Cx. pipiens biotypes in The Netherlands, mosquitoes were collected with traps and with the help of the general public (chapter 2). Both biotypes and their hybrids were found year-round throughout The Netherlands, in indoor as well as outdoor locations. Biotype molestus was mainly responsible for indoor biting nuisance during summer and winter. Hybridization readily occurred between both biotypes resulting in overall hybridization rates of up to $15 \%$. Thus, both $C x$. pipiens biotypes and hybrids are present throughout The Netherlands, with year-round biting activity of biotype molestus and hybrids.

The next step was to investigate spatial variation in the composition of $C x$. pipiens populations at the European scale (chapter 3). A comparison was made between the biotypes and hybrids collected with traps in three different habitat types (farm, peri-urban, and wetland), at three different latitudes (Sweden, The Netherlands, and Italy) in Europe. The Cx. pipiens population composition differed across habitat types, but differences were specific for each of the countries. A more clear effect was found across the different latitudes. The biotype composition shifted towards increasing biotype pipiens from southern to northern Europe. In Sweden, the Cx. pipiens population was dominated by biotype pipiens, whereas in Italy the ratio between biotype pipiens and molestus was more equal. In addition, similar hybridization rates were found in The Netherlands and Italy. Thus, Cx. pipiens biotype composition shifts towards more biotype pipiens at northern latitudes, but with similar hybridization 
rates in the sampling locations in The Netherlands and Italy.

Now that we know that the $C x$. pipiens biotypes and their hybrids are present throughout The Netherlands, we set out to determine their vector competence for WNV (chapter 4). Biotype pipiens, biotype molestus, and hybrids originating from The Netherlands were tested for their ability to transmit WNV after 14 days incubation at a temperature of $18{ }^{\circ} \mathrm{C}, 23^{\circ} \mathrm{C}$, or $28^{\circ} \mathrm{C}$, under controlled laboratory conditions. The biotypes responded differentially to temperature, with increasing transmission rates of biotype pipiens and hybrids at higher temperatures. Transmission rates of biotype molestus of approximately $10 \%$ were constant across the temperature range from $18{ }^{\circ} \mathrm{C}$ to $28{ }^{\circ} \mathrm{C}$. Temperature was identified as an important limiting factor for WNV transmission, with very low transmission rates of $0 \%$ for biotype pipiens and $2 \%$ for hybrids, after 14 days of incubation at $18{ }^{\circ} \mathrm{C}$. In contrast, one out of three biotype pipiens mosquitoes was able to transmit WNV after 14 days of incubation at 28 ${ }^{\circ} \mathrm{C}$. Thus, the $\mathrm{Cx}$. pipiens biotypes originating from The Netherlands are competent vectors for WNV, but low temperature is an importing limiting factor for transmission.

To assess differences in vector competence among mosquito populations originating from northern and southern Europe, a direct comparison was made between a Dutch and Italian Cx. pipiens biotype pipiens population (chapter 5). Vector competence was tested after 14 days incubation at a temperature of $18^{\circ} \mathrm{C}, 23^{\circ} \mathrm{C}$, or $28^{\circ} \mathrm{C}$. Both populations had similar transmission rates ranging between $0 \%$ and $16 \%$. This suggests that there are no intrinsic differences in vector competence between the selected Dutch and Italian population. Low temperature was again confirmed as an important limiting factor for WNV transmission by both populations.

WNV transmission is highly dependent on the feeding behaviour of mosquitoes. It would be beneficial for WNV if infection of mosquitoes with WNV would result in increased virus transmission. To investigate whether WNV can increase its transmission by influencing the behaviour of its mosquito vector, the effect of WNV on the mosquito's host-seeking behaviour and performance was investigated (chapter 6). Infection with WNV resulted in a decreased host-seeking response, which could not be attributed to a decreased odour perception by the antennae. Interference of WNV with the mosquito brain was, therefore, hypothesized as a potential underlying mechanism. In addition, WNV did not influence the mosquito's flight ability, survival, or blood-feeding behaviour. Thus, WNV decreases the host-seeking response of its mosquito vector, but does not influence the mosquito's performance.

Previous chapters of this thesis indicated that the contribution of the $C x$. pipiens biotypes to WNV transmission may be very different. To assess risks of WNV establishment in Europe, a basic reproduction number model was developed, by including the biotype-specific and temperature-dependent effects that were elucidated 
in earlier thesis chapters (chapter 7). Based on model outcomes, temperature, $C x$. pipiens population composition, and mosquito-to-host ratio were identified as important factors for the risk of WNV establishment. Elasticity analysis showed that biotype pipiens was the main contributor to the risk of WNV establishment, which highlights the importance of differentiating mosquitoes to the biotype level. Thus, higher temperatures, shifts in population composition towards biotype pipiens, and higher mosquito-to-host ratios may increase the risk of WNV establishment in northern Europe.

Until recently, it was unknown whether European mosquitoes were capable of transmitting WNV. However, during the past years, several studies on vector competence of European mosquito species have been published. Vector competence for WNV of mosquito species across Europe was compared, and underlying mechanisms of vector competence for WNV were discussed (chapter 8). Considerable variation exists among study protocols, which hampers the ability to make direct comparisons between outcomes of studies. Nevertheless, several mosquito species from across Europe are competent vectors for WNV. Thus, WNV surveillance is recommended not only for southern and central Europe, but also for northern European countries. Studying the underlying mechanisms of vector competence for WNV is an important goal to increase our understanding of the intrinsic factors that determine vector competence.

This thesis contributes to our understanding of differences in WNV circulation between northern and southern Europe, by focusing on the ecology of mosquitovirus interactions. Three key mosquito-related factors were identified which may explain the absence of WNV outbreaks in northern Europe (chapter 9). Culex pipiens abundance, vector competence, and the duration of the infectious lifetime are all relatively lower or reduced in northern Europe compared to southern Europe, as a result of the cooler northern European climate. Thus, low temperature is an important limiting factor for WNV establishment in northern Europe, due to its negative effect on the three key mosquito-related factors. Nevertheless, the data presented in this thesis also show that the $C x$. pipiens biotypes and their hybrids are present throughout The Netherlands, and that they are competent vectors for WNV under favourable climatic conditions. When considering the outcomes of this thesis in the light of climate change, northern European countries such as The Netherlands should be prepared for future WNV transmission. Thus, WNV surveillance, mosquito monitoring, and awareness among the general public are needed in northern Europe to be prepared for WNV transmission. 



\section{Samenvatting}


Het West-Nijl virus (WNV) is een door muggen overdraagbaar virus dat zorgt voor herhaaldelijke uitbraken in mensen en paarden in Zuidoost-Europa. In Noord-Europa zijn er echter nog geen gevallen van WNV infectie in mensen gedetecteerd. In de natuur circuleert WNV tussen muggen en vogels. Mensen en paarden kunnen ook geïnfecteerd raken met WNV, wat tot ziekte kan leiden, maar het virus kan zich in het bloed van zoogdieren niet genoeg vermeerderen om verder verspreid te worden naar muggen. Muggen spelen een belangrijke rol in de verspreiding van WNV door hun specifieke voorkeur voor bepaalde gastheren, vectorcompetentie, en door hun afhankelijkheid van omgevingsfactoren zoals temperatuur. Mug-gerelateerde factoren kunnen daardoor mogelijk de afwezigheid van WNV uitbraken in NoordEurope verklaren. Het doel van dit proefschrift was om de rol van Culex (Cx.) pipiens muggen in de overdracht van WNV in Europa te onderzoeken, en om verschillen in WNV circulatie tussen Noord- en Zuidoost-Europa te begrijpen. Dit proefschrift richt zich op muggen als hoofdrolspelers in de overdracht van WNV, door de interactie tussen mug, virus, en temperatuur te onderzoeken.

Een van de belangrijkste vectoren voor WNV is de noordelijke huissteekmug ( $C x$. pipiens), een soort die bestaat uit twee verschillende biotypen, pipiens en molestus. Beide biotypen zijn morfologisch identiek, maar verschillen in hun gedrag, zoals hun voorkeur voor gastheren. Kruisingen tussen beide biotypen resulteren in hybriden die ideale eigenschappen hebben om WNV van vogels naar mensen over te dragen, doordat ze zowel vogels als zoogdieren bijten. Om inzicht te krijgen in de ruimtelijke verspreiding en de activiteit van de $C x$. pipiens biotypen in Nederland, hebben we muggen verzameld met muggenvallen en met hulp van Nederlandse burgers (hoofdstuk 2). Beide biotypen en hybriden werden gedurende het hele jaar gevonden op verschillende plekken in Nederland, zowel binnenshuis als buitenshuis. Kruisingen tussen de biotypen werden in het hele land gevonden, met percentages tot wel $15 \%$ hybriden in de muggenpopulatie. Kortom, beide $C x$. pipiens biotypen en hun hybriden zijn aanwezig in Nederland, en zowel biotype molestus als hybriden zijn in de zomer en winter actief.

De volgende stap in het onderzoek was om de samenstelling van de $C x$. pipiens muggenpopulaties te onderzoeken binnen Europa (hoofdstuk 3 ). Een vergelijking werd gemaakt tussen biotypen en hybriden gevangen in drie verschillende leefgebieden (ruraal gebied, bebouwd gebied, en nat natuurgebied), in drie verschillende Europese landen (Zweden, Nederland, en Italië). De muggenpopulaties verschilden tussen de leefgebieden, maar deze verschillen waren specifiek voor ieder land. Een duidelijker patroon werd gevonden tussen de verschillende landen. Van Zuid- naar Noord-Europa nam de aanwezigheid van biotype pipiens in de muggenpopulatie sterk toe. In Zweden werden bijna alleen maar pipiens muggen gevonden, terwijl de ratio tussen biotype pipiens en molestus in Italië ongeveer gelijk was. Daarnaast 
was het percentage hybriden in de muggenpopulatie ongeveer gelijk in Nederland en Italië. Kortom, het aandeel van biotype pipiens in de Cx. pipiens muggenpopulatie neemt sterk toe van Zuid- naar Noord-Europa, maar met een relatief gelijk aandeel hybriden in Nederland en Italië.

Nu we weten dat de Cx. pipiens biotypen en hybriden aanwezig zijn in Nederland, hebben we onderzocht hoe goed ze in staat zijn om WNV over te dragen (hoofdstuk 4). Met andere woorden, we onderzochten de vectorcompetentie van de Nederlandse $C x$. pipiens muggen. Vectorcompetentie voor WNV van zowel biotype pipiens, biotype molestus, en hybriden voor WNV werd getest in het laboratorium, na 14 dagen incubatie bij $18{ }^{\circ} \mathrm{C}, 23^{\circ} \mathrm{C}$, of $28^{\circ} \mathrm{C}$. Vectorcompetentie van de biotypen was sterk verschillend bij de geteste temperaturen: bij toenemende temperaturen was de transmissie van WNV door biotype pipiens en hybriden hoger, terwijl de transmissie van WNV door biotype molestus ongeveer gelijk bleef bij alle drie de temperaturen. Daarnaast was temperatuur een belangrijke limiterende factor voor de overdracht van WNV. Geen van de biotype pipiens muggen, en slechts $2 \%$ van de hybriden konden WNV overdragen na 14 dagen incubatie bij $18^{\circ} \mathrm{C}$. Daarentegen kon $1 \mathrm{op}$ de 3 biotype pipiens muggen WNV overdragen na 14 dagen incubatie bij $28^{\circ} \mathrm{C}$. Dit laat zien dat Nederlandse $C x$. pipiens muggen WNV kunnen overdragen, maar dat lage temperatuur een belangrijke limiterende factor is voor de overdracht van WNV.

Om mogelijke verschillen in vectorcompetentie tussen een Noord- en ZuidEuropese muggen populatie te onderzoeken, werd een directe vergelijking gemaakt tussen vectorcompetentie van een Nederlandse en Italiaanse $C x$. pipiens biotype pipiens populatie (hoofdstuk 5 ). Vectorcompetentie werd wederom getest na een incubatieperiode van 14 dagen bij $18^{\circ} \mathrm{C}, 23^{\circ} \mathrm{C}$, of $28^{\circ} \mathrm{C}$. Er werden geen verschillen gevonden tussen vectorcompetenties van beide muggenpopulaties. Het percentage muggen van beide populaties dat WNV kon overdragen varieerde tussen $0 \%$ en 16 $\%$, afhankelijk van de incubatietemperatuur. Dit laat zien dat er ogenschijnlijk geen intrinsieke verschillen zijn tussen beide muggenpopulaties. Opnieuw werd bevestigd dat lage temperaturen een belangrijke limiterende factor voor WNV overdracht zijn.

De overdracht van WNV is sterk afhankelijk van het zoekgedrag naar gastheren door muggen. Voor WNV zou het gunstig zijn als infectie van muggen met WNV leidt tot verhoogde overdracht van het virus. Om te onderzoeken of WNV haar eigen transmissie kan verhogen door het gedrag van de mug te beïnvloeden, werd de invloed van WNV op het zoekgedrag van muggen naar gastheren onderzocht (hoofdstuk 6). Infectie met WNV resulteerde in een verminderde reactie van de mug op de geur van gastheren, terwijl de mug nog steeds in staat was om geuren waar te nemen met haar antennen. Een mogelijke verklaring voor de verminderde reactie op geuren van gastheren is interferentie van WNV in de hersenen van de mug. 
Daarnaast was er geen effect van WNV-infectie op het vlieggedrag, overleving, of bloedvoedingsgedrag van de mug. De conclusie die uit deze experimenten getrokken kan worden is dat WNV de reactie van de mug op gastheren vermindert, maar dat infectie geen invloed heeft op andere eigenschappen van de mug.

Vorige hoofdstukken van dit proefschrift hebben laten zien dat de bijdrage van de $C x$. pipiens biotypen aan de overdracht van WNV mogelijk verschillend zou kunnen zijn. Om de risico's op overdracht van WNV in Europa in te schatten, werd er een model ontwikkeld waarbij rekening gehouden werd met biotype-specifieke en temperatuursafhankelijke factoren zoals beschreven in voorgaande hoofdstukken (hoofdstuk 7). Gebaseerd op de uitkomsten van het model werden de factoren temperatuur, samenstelling van de muggenpopulatie, en de ratio tussen muggen en gastheren geïdentificeerd als factoren die belangrijk zijn voor het vestigen van WNV in een bepaald gebied. Verdere analyses lieten zien dat biotype pipiens de belangrijkste bijdrage levert aan overdracht van WNV. Dit laat duidelijk het belang zien van het maken van onderscheid tussen de biotypen. Kortom, hogere temperaturen, verschuivingen in de samenstelling van de muggenpopulatie, en hogere ratio's tussen muggen en gastheren kunnen mogelijk het risico op overdracht van WNV in Noord-Europa verhogen.

Tot voor kort was het niet duidelijk of Europese muggen in staat waren om WNV over te dragen. Gedurende de afgelopen jaren zijn er echter verschillende studies over de vectorcompetentie van Europese muggensoorten gepubliceerd. Een vergelijking van deze studies naar vectorcompetentie van Europese muggensoorten voor WNV werd gemaakt, en daarnaast werden onderliggende mechanismen bediscussieerd (hoofdstuk 8). Er bestaat aanzienlijke variatie tussen protocollen om vectorcompetentie vast te stellen, wat het lastig maakt om directe vergelijkingen te maken tussen verschillende studies. Ondanks deze variatie wordt wel duidelijk dat verschillende muggensoorten in Europa in staat zijn om WNV over te dragen. Het monitoren van WNV is daarom niet alleen nodig in Zuidoost-Europa, maar is ook geadviseerd voor Noord-Europa. Het bestuderen van onderliggende mechanismen van vectorcompetentie voor WNV is belangrijk om onze kennis over factoren die bepalend zijn voor vectorcompetentie beter te begrijpen.

Dit proefschrift draagt bij aan onze kennis over verschillen in WNV circulatie tussen Noord- en Zuidoost-Europa, door zich te richten op de interactie tussen mug en virus. Drie belangrijke factoren werden geïdentificeerd die de afwezigheid van WNV uitbraken in Noord-Europa kunnen verklaren (hoofdstuk 9). De dichtheid van muggen, vectorcompetentie, en de duur van de infectieuze levensduur van muggen zijn allen lager of gereduceerd in Noord-Europa in vergelijking met Zuidoost-Europa. Dit als gevolg van het koudere klimaat in Noord-Europa. Kortom, lage temperaturen zijn 
een belangrijke limiterende factor voor de vestiging van WNV in Noord-Europa, door de negatieve invloed op de drie genoemde mug-gerelateerde factoren. Desondanks laten de resultaten in dit proefschrift ook zien dat de $C x$. pipiens biotypen en hybriden veel voorkomen in Nederland, en dat ze in staat zijn om WNV over te dragen bij gunstige klimaatomstandigheden. Deze uitkomsten in combinatie met de voorspellingen voor klimaatverandering laten zien dat Noord-Europese landen zoals Nederland voorbereid moeten zijn op circulatie van WNV in de nabije toekomst. Monitoren van WNV, het in kaart brengen van het vóórkomen van muggen, en het bewustzijn van mogelijke WNV-circulatie in Noord-Europa zijn nodig om voorbereid te zijn op overdracht van WNV in de toekomst. 

Acknowledgements 
Now that my PhD project has come to an end I can look back to a great start of my scientific career. During the past 4 years I have gained a lot of knowledge, skills, and experience for which I am very grateful. My PhD would not have run so smoothly without the help and support of a great group of people around me. For that I would like to thank all my colleagues at the Laboratories of Entomology and Virology, collaborators, friends, and family.

Allereerst wil ik Sander bedanken voor de uitstekende begeleiding gedurende de afgelopen jaren. Het begon allemaal met een BSc thesis over risico's van mugoverdraagbare ziekten voor Nederland. Wie had toen gedacht dat ik 3 jaar later zelf onderzoek zou gaan doen naar het risico van West-Nijl virus voor Nederland? Ik ben ontzettend dankbaar dat je mij de kans hebt gegeven om aan dit PhD project te werken. Ik kan niet anders zeggen dan dat ik geen betere begeleider had kunnen hebben: altijd vol enthousiasme, vernieuwende ideeën, en zonder jou was mijn $\mathrm{PhD}$ zeker niet zo soepel verlopen! Ik ben blij dat onze samenwerking nog doorgaat in mijn huidige postdoc onderzoek, en ik weet zeker dat we in de toekomst nog veel vaker zullen samenwerken aan uitdagende projecten.

Marcel, ook jou wil ik hartelijk bedanken voor de kans die je me 4 jaar geleden geboden hebt. Ik heb veel bewondering voor de manier waarop je onze leerstoelgroep aanstuurt. Ik ben dankbaar voor de waardevolle discussies en (razendsnelle) feedback op mijn werk. Door jouw vernieuwende en scherpe blik werd mijn werk altijd weer beter. Ik hoop dat onze wegen elkaar nog vaak zullen kruisen in de toekomst, en ik ben blij dat ik mijn wetenschappelijke carrière nog even kan voortzetten bij Entomologie.

Gorben, ondanks dat jij geen formele rol in de begeleiding van mijn $\mathrm{PhD}$ had, heb je wel een grote rol gespeeld. Dit is in een oogopslag te zien aan het aantal publicaties waarop jij vermeld staat als coauteur. Ik ben je heel erg dankbaar voor de input tijdens vele discussies. De samenwerking tussen Entomologie en Virologie is een gouden formule, en ik ben ervan overtuigd dat dit succes in de toekomst voortgezet gaat worden.

Giel, ook jij hebt een belangrijke rol gespeeld in mijn PhD. Samen vormen we een ontzettend goed team, waardoor we al vele successen op onze naam hebben staan. De kracht ligt ook hier in de perfecte aanvulling van jouw virologische kennis op mijn entomologische achtergrond. Naast het harde werk was het altijd gezellig om samen met jou in het BSL3 lab te werken, vooral de speeksel-afname van honderden muggen was elke keer weer een feest! Onze samenwerking gaat hier zeker nog niet stoppen en ik kijk er naar uit om samen met jou nieuwe voorstellen en papers te schrijven. 
Corinne, ook met jou heb ik de afgelopen jaren met veel plezier samengewerkt! Alle lof voor de georganiseerde manier waarop jij het BSL3 lab draaiende houdt! Mijn dank is groot voor de aanlevering van de vele cellen en virussen die ik tijdens mijn experimenten gebruikt heb.

Jelke, het is alweer even geleden dat jij het lab verlaten hebt, maar ik wil jou ook zeker bedanken voor al je hulp tijdens het opstarten van mijn PhD. Door jouw training en begeleiding had ik me geen betere start van het BSL3 werk kunnen wensen. Ik ben daarom blij dat je weer terugkeert naar Wageningen, en dat we onze samenwerking kunnen voortzetten.

Els, bedankt voor je hulp bij het opzetten van het BSL3 lab, en voor alle adviezen over veiligheid.

Arnold, bedankt voor je hulp bij het verwezenlijken van muggenradar. Zonder jouw kennis en ervaring met citizen science was het nooit zo'n groot succes geworden!

Jan, samen met jou hebben we een heel leuke verdiepende studie in Amsterdam gedaan. Bedankt voor je enthousiasme en betrokkenheid.

Ik wil Adolfo en Arjan bedanken voor alle hulp en goede adviezen. De analyse van de door jullie verzamelde muggen vormt een belangrijk onderdeel van het tweede hoofdstuk in dit proefschrift.

Marcel W., bedankt voor je hulp bij het ontwikkelen van de moleculaire test om Culex pipiens muggen te identificeren. Dit was een belangrijke eerste stap in mijn $\mathrm{PhD}$ project.

Wietse, jij heel erg bedankt voor het verzamelen van molestus muggen en larven. Zonder jouw bijdrage had ik nooit zulke goede muggenlijnen op kunnen zetten in het lab.

Tim, bedankt voor alle (Brabantse!) gezelligheid en de fijne samenwerking. Door jouw harde werk in het veld, waarbij je vele muggen verzameld hebt in verschillende Europese landen, hebben we samen een mooie paper kunnen schrijven. Dit proces verliep zo soepel dat het bijna leek alsof deze paper er vanzelf gekomen is. Ik ben dan ook blij dat we onze samenwerking voorzetten in het knuttenproject. Ook bedankt dat je een van mijn paranimfen bent.

Guido, Claudia, and Uno, many thanks for you input and support with the fieldwork in Italy and Sweden!

Joop en Hans, bedankt voor jullie adviezen en input in de EAG studies.

Gerrit, heel erg bedankt voor je input en adviezen over de statistiek die ik in dit proefschrift heb toegepast. 
Nienke, jij bent echt een fantastische modelleur! Zonder jouw hulp had hoofdstuk 7 in dit proefschrift nooit tot stand kunnen komen. Bedankt voor de fijne samenwerking! Jeroen en Rianka, het Rift Valley fever project waarin we samenwerken is geen onderdeel van dit proefschrift, maar toch wil ik ook jullie bedanken voor de fijne samenwerking. Ik heb ontzettend veel van jullie geleerd, en ben dankbaar dat ik bij jullie in Lelystad mee mocht lopen in de high containment unit.

I would like to thank all my colleagues at the Laboratory of Entomology for all the fun, great times, and fruitful discussions that we had during the past years. I very much enjoyed all coffee breaks, labuitjes, We-days, drinks, and parties.

My special thanks go to the vector group: Sander, Willem, Niels, Jetske, Jeroen, Alex, Rob, Léon, Claudio, Tobias, Gilian, David, Tim, Jeanine, Florian, Emmanuel, Annette, Marilyn, and Monicah. Thanks for the great atmosphere and many fruitful discussions.

Also I would like to thank all the students that I supervised during the past 4 years: Lennart, Bram, Tessa, Steffanie, Linda, Diede, Ellis, and Michelle. Many thanks for your input and contribution to my research!

Lucille, thank you for all the fun times at Ento! With you sitting at the desk opposite to me, there was no day that passed without a smile. Also thank you for being my paranymph. Jenny, Daan, Maite, and Sandeep, thank you all for being such nice desk mates as well. Tim S. het was van korte duur maar met jou als achterbuurman was het altijd gezellig! Gelukkig kreeg je een leuke opvolger, Margot, ook jij bedankt voor de gezelligheid! Dennis, bedankt voor de vele leuke gesprekken en je altijd wijze raad. Karol \& Katherine, thank you for all the nice dinners and fun activities! Keiko, bedankt voor je gezelligheid! Ik heb genoten van de Nederlandse lunches en Japanse lessen. Julia, thank you for the nice conversations and fun times during zumba. Antonio, thank you for your Italian jokes, and for always making me smile with Nutélla! Claudio \& Lidia, thank you for the nice game nights!

Daarnaast wil ik een aantal mensen bedanken die een ontzettend belangrijke rol vervullen binnen onze groep. Angelique, bedankt voor alle administratieve hulp gedurende de afgelopen jaren. Patrick, bedankt voor je gezelligheid en alle ondersteuning en hulp in het moleculaire lab. Léon, Frans, en Andre, bedankt voor jullie inzet in de muggenkweek.

During my PhD, I have been submerged in the world of Virology. Colleagues at the Laboratory of Virology, many thanks for the interesting discussions and the good times! 
I would like to thank the PE\&RC council, Wageningen PhD council, and Wageningen PhD symposium committee 2015, for the great collaboration and good times we had during the last years. Thanks to you all I was able to further develop my organisational and leadership skills. Special thanks go to Claudius and Lennart. Thank you for your supervision and the effort you put in PE\&RC.

Ik wil Iris, Angela, Kim, Marlie, Renske, Anhilde, Hester, en Eefke bedanken voor alle gezelligheid en leuke uitjes van de afgelopen jaren!

Ook wil ik mijn hele familie heel erg bedanken voor jullie interesse in mijn onderzoek. Er ging geen verjaardag of familiebarbecue voorbij zonder de welbekende vraag: "hoe gaat het met de muggen?". Daarnaast wil ik ome Andre en tante Dinie speciaal bedanken voor alle muggeneitjes die ik samen met mama uit jullie regentonnen heb mogen vissen. Deze eitjes waren essentieel om muggen te kunnen kweken voor mijn experimenten.

Ten slotte wil ik mijn ouders en mijn broer Stefan bedanken voor jullie onvoorwaardelijke steun. Zonder jullie was ik nooit zover gekomen en had ik dit niet kunnen bereiken! 

Curriculum vitae 


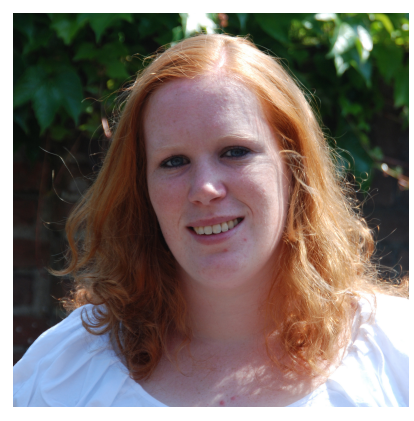

Chantal Vogels was born on 4 May 1989 in Best, The Netherlands. After attending secondary school at the Heerbeeck college in Best, she started her studies in Biology at Wageningen University in 2007. During her MSc thesis she evaluated the entomopathogenic fungus Beauveria bassiana for the control of malaria mosquitoes. During a second MSc thesis she investigated the effects of habitat fragmentation and hunting on the terrestrial mammal community composition in Central Panama. In addition, she did an internship at the National Institute for Public Health and the Environment (RIVM) to study resistance of head lice against insecticides, for which she had to rear head lice on her arm for several months. In 2013, she started her $\mathrm{PhD}$ project at the Laboratory of Entomology, Wageningen University \& Research, under supervision of prof. Marcel Dicke and dr. Sander Koenraadt. Within this project, she studied the role of Culex pipiens mosquitoes in transmission of West Nile virus in Europe. She performed extensive vector competence experiments with Culex pipiens mosquitoes infected with West Nile virus, under strict safety measures in the biosafety level 3 laboratory. The results of this research are presented in this thesis. In addition to the studies with West Nile virus, she was also involved in studies on transmission of Zika virus and chikungunya virus by Aedes aegypti mosquitoes, Schmallenberg virus by Culicoides biting midges, and Rift Valley fever virus by Culex pipiens mosquitoes. During her $\mathrm{PhD}$, she was an active member and chair of the PE\&RC PhD council, secretary of the Wageningen PhD council, and organised the Wageningen PhD symposium in 2015. Chantal was awarded the Storm-van der Chijs stipend for most talented female PhD candidates in May 2017. After completion of her PhD thesis, she continued with post-doctoral research at the Laboratory of Entomology, Wageningen University and Research. Within this project, she will study factors that influence the vector competence of mosquitoes for Zika virus. After this postdoc, Chantal has the ambition to pursue her scientific career abroad, by continuing to research virus-vector interactions. 



\section{List of publications}




\section{Published papers}

Vogels, C.B.F., Fros, J.J., Pijlman, G.P., van Loon, J.A., Gort, G., Koenraadt, C.J.M. 2017. Virus interferes with host-seeking behaviour of mosquito. Journal of Experimental Biology (in press). (Chapter 6 in this thesis)

Vogels, C.B.F., Göertz, G.P., Pijlman, G.P., Koenraadt, C.J.M. 2017. Vector competence of northern and southern European Culex pipiens pipiens mosquitoes for West Nile virus across a gradient of temperatures. Medical and Veterinary Entomology (in press). (Chapter 5 in this thesis)

Vogels*, C.B.F., Hartemink*, N., Koenraadt, C.J.M. 2017. Modelling West Nile virus transmission risk in Europe: effect of temperature and mosquito biotypes on the basic reproduction number. Scientific Reports 7:5022. (Chapter 7 in this thesis)

Göertz*, G.P., Vogels*, C.B.F., Geertsema, C., Koenraadt, C.J.M., Pijlman, G.P. 2017. Mosquito coinfection with Zika and chikungunya virus allows simultaneous transmission without affecting the vector competence of Aedes aegypti. PLoS Neglected Tropical Diseases 11:e0005654.

Vogels*, C.B.F., Möhlmann*, T.W.R., Favia, G., Wennergren, U., Koenraadt, C.J.M. 2016. Latitudinal diversity of Culex pipiens biotypes and hybrids in farm, peri-urban, and wetland habitats in Europe. PLoS One 11:e0166959. (Chapter 3 in this thesis)

Vogels, C.B.F., Fros, J.J., Göertz, G.P., Pijlman, G.P., Koenraadt, C.J.M. 2016. Vector competence of northern European Culex pipiens biotypes and hybrids for West Nile virus is differentially affected by temperature. Parasites \& Vectors 9:393. (Chapter 4 in this thesis)

Göertz, G.P., Fros, J.J., Miesen, P., Vogels, C.B.F., van der Bent, M.L., Geertsema, C., Koenraadt, C.J.M., van Rij, R.P., van Oers, M.M., Pijlman, G.P. 2016. Non-coding subgenomic flavivirus RNA is processed by the mosquito RNAi machinery and determines West Nile virus transmission by Culex pipiens mosquitoes. Journal of Virology 90:10145-10159.

Vogels, C.B.F., van de Peppel, L.J.J., van Vliet, A.J.H., Westenberg, M., Ibañez-Justicia, A., Stroo, A., Buijs, J.A., Visser, T.M., Koenraadt, C.J.M. 2015. Winter activity and aboveground hybridization between the two biotypes of the West Nile virus vector Culex pipiens. VectorBorne and Zoonotic Diseases 15:619-626. (Chapter 2 in this thesis)

Fros, J.J., Geertsema, C., Vogels, C.B.F., Roosjen, P.P., Failloux, A-B., Vlak, J.M., Koenraadt, C.J.M., Takken, W., Pijlman, G.P. 2015. West Nile virus: High transmission rate in northwestern European mosquitoes indicates its epidemic potential and warrants increased surveillance. PLoS Neglected Tropical Diseases 9:e0003956.

Fros, J.J., Miesen, P., Vogels, C.B.F., Gaibani, P., Sambri, V., Martina, B.E., Koenraadt, C.J.M., van Rij, R.P., Vlak, J.M., Takken, W., Pijlman, G.P. 2015. Comparative Usutu and West Nile virus transmission potential by local Culex pipiens mosquitoes in north-western Europe. One Health 1:31-36.

Meyer, N.F.V., Esser, H.J., Moreno, R., van Langevelde, F., Liefting, Y., Ros Oller, D., Vogels, C.B.F., Carver, A.D., Nielsen, C.K., Jansen, P.A. 2015. An assessment of the terrestrial mammal communities in forests of Central Panama, using camera-trap surveys. Journal for Nature Conservation 26:28-35.

Vogels, C.B.F., Bukhari, T., Koenraadt, C.J.M. 2014. Fitness consequences of larval exposure to Beauveria bassiana on adults of the malaria vector Anopheles stephensi. Journal of Invertebrate Pathology 119:19-24.

\footnotetext{
* These authors contributed equally to this work
} 


\section{Submitted papers}

Vogels*, C.B.F., Göertz*, G.P., Pijlman, G.P., Koenraadt, C.J.M. Vector competence of European mosquitoes for West Nile virus. Submitted. (Chapter 8 in this thesis)

Vloet, R.P.M., Vogels, C.B.F., Koenraadt, C.J.M., Pijlman, G.P., Eiden, M., van Keulen, L.J.M., Wichgers Schreur, P.J., Kortekaas, J. Transmission of Rift Valley fever virus from Europeanbreed lambs to Culex pipiens mosquitoes. Submitted.

\section{Other publications}

Van Vliet, A.J.H., Bron, W., Koenraadt, C.J.M., Vogels, C.B.F. Door droogte minder muggenoverlast? Nature Today: 22-06-2017.

van Vliet, A.J.H., Vogels, C.B.F., Koenraadt, C.J.M. Muggenradar onderdeel van nieuw baanbrekend wereldwijd citizen science programma voor muggen. Nature Today: 08-05-2017.

van Vliet, A.J.H., Bron, W., Koenraadt, C.J.M., Vogels, C.B.F. Heeft u muggenoverlast met Pasen? Nature Today: 17-04-2017.

van Vliet, A.J.H., Koenraadt, C.J.M., Vogels, C.B.F. Zuiden Nederland meer muggen en meer overlast. Nature Today: 26-08-2016.

van Vliet, A.J.H., Koenraadt, C.J.M., Vogels, C.B.F., de Kind, N. Weersomstandigheden ideaal voor ontwikkeling van steekmuggen; meld mate van overlast. Nature Today: 29-06-2016.

Vogels, C.B.F., Koenraadt, C.J.M., van Vliet, A.J.H. Muggenradar. Dierplagen informatie: 05-022016.

van Vliet, A.J.H., Bemelmans, E., Vogels, C.B.F., Koenraadt, C.J.M. Huissteekmug in stad bijt vaker dan op platteland. Nature Today: 24-01-2016.

Koenraadt, C.J.M., Möhlmann, T.W.R., Vogels, C.B.F., Steen, W. Bunkers nieuwe Hollandse waterlinie vol met steekmuggen. Nature Today: 13-03-2015.

Koenraadt, C.J.M., Vogels, C.B.F., van Vliet, A.J.H. Wageningen University wil weer dode steekmuggen. Nature Today: 05-02-2015.

Buijs, J.A., Cianci, D., Koenraadt, C.J.M., Stroo, A., Vogels, C.B.F. 2015. Characterizing mosquito breeding sites in an urban setting: a case study in Amsterdam. Chapter 6 in PhD thesis Daniela Cianci.

Vogels, C.B.F., Koenraadt, C.J.M., van Vliet, A.J.H. Overlast in zomer ook door tweelingzusje huissteekmug. Nature Today: 19-12-2014.

Koenraadt, C.J.M., Vogels, C.B.F., van Vliet, A.J.H., Bron, W. Welke mug veroorzaakt de meeste overlast? Nature Today: 28-08-2014

Koenraadt, C.J.M., Vogels, C.B.F., van Vliet, A.J.H. Mug heeft tweelingzusje dat in winter voor overlast zorgt. Nature Today: 11-05-2014.

van Vliet, A.J.H., Bron, W., Koenraadt, C.J.M., Vogels, C.B.F., van de Peppel, L.J.J. 55 procent van naar Muggenradar opgestuurde muggen is steekmug. Nature Today: 26-01-2014.

Koenraadt, C.J.M., Vogels, C.B.F., van de Peppel, L.J.J., van Vliet, A.J.H., Bron, W. Wageningse onderzoekers op zoek naar winterse steekmuggen. Nature Today: 11-01-2014.

Vogels, C.B.F., Dam-Deisz, W.D.C., Reusken, C.B.E.M., Sprong, H., Beaujean, D., Tijsse-Klasen, E., Braks, M.A.H. 2012. Is resistance lousing things up in The Netherlands? Proceedings of the Netherlands Entomological Society Meeting, volume 23. 

Training and education statement 


\section{PE\&RC Training and Education Statement}

With the training and education activities listed below the $\mathrm{PhD}$ candidate has complied with the requirements set by the C.T. de Wit Graduate School for Production Ecology and Resource Conservation (PE\&RC) which comprises of a minimum total of 32 ECTS (= 22 weeks of activities)

\section{Review of literature (6 ECTS)}

- Vector competence of European mosquitoes for West Nile virus

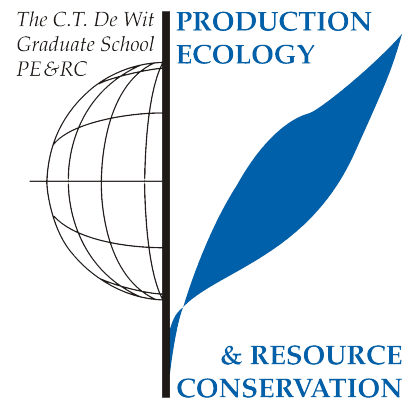

\section{Writing of project proposal (4.5 ECTS)}

- The role of environmental, behavioural, and genetic factors in West Nile virus transmission by European Culex mosquitoes

\section{Post-graduate courses (3.9 ECTS)}

- Introduction to R for statistical analysis; PE\&RC (2013)

- Mosquito Informatics (INFRAVEC); European Bioinformatics Institute, Hinxton, United Kingdom (2014)

- $\quad$ Linear models; PE\&RC and SENSE (2015)

- Generalized linear models; PE\&RC and SENSE (2015)

- Training course on vector ecology and control of mosquitoes; European Society for Vector Ecology, Lisbon, Portugal (2016)

- $\quad$ Mixed models; PE\&RC and SENSE (2017)

\section{Laboratory training and working visits (2.7 ECTS)}

- Laboratory biosafety and biosecurity; RIVM (2014)

- $\quad$ Emergency response training (BHV); WUR (2013-2017)

Invited review of (unpublished) journal manuscript (2 ECTS)

- Tropical Medicine and International Health: feeding behaviour Culex mosquitoes (2015)

- Journal of Environmental Research and Pubic Health: entomological surveillance of vector-borne diseases (2016)

Deficiency, refresh, brush-up courses (6 ECTS)

- Molecular virology; Laboratory of Virology (2013)

\section{Competence strengthening / skills courses (3.7 ECTS)}

- $\quad$ Techniques for writing and presenting a scientific paper; WGS (2014)

- $\quad$ Reviewing a scientific paper; WGS (2015)

- $\quad$ PhD course carousel; WGS (2015)

- $\quad$ Last stretch of the PhD programme; WGS (2016)

- T-week: technical course for developmental workers; WOT, TU, Twente (2016)

- $\quad$ Adobe InDesign essential training; WUR Library/Agile color (2016)

PE\&RC Annual meetings, seminars and the PE\&RC weekend (3.3 ECTS)

- $\quad$ PE\&RC First years weekend (2013)

- PE\&RC Midterm weekend (2015) 
- $\quad$ PE\&RC Last years weekend (2016)

- PE\&RC Day (2013-2016)

Discussion groups / local seminars / other scientific meetings (13.4 ECTS)

- Annual meetings of entomologists in the Netherlands; oral presentations (2013-2016)

- Wageningen Evolution and Ecology seminars (WEES) (2013-2017)

- Vector Ecology meetings; oral presentations (2013-2017)

- Netherlands Annual Ecology meeting; oral presentation (2015)

- Wageningen PhD Symposium; organizer, convener, and oral presentation (2015-2016)

- $\quad$ Dutch arboviral network workshop; organizer and oral presentation (2016-2017)

- $\quad$ Netherlands Centre for One Health masterclass (2017)

\section{International symposia, workshops and conferences (6.1 ECTS)}

- International meeting on arboviruses and their vectors, Glasgow, United Kingdom; poster presentation (2015)

- European Society for Vector Ecology conference, Lisbon, Portugal; oral presentation (2016)

- European Mosquito Control Association conference, Bečići, Montenegro; oral presentation (2017)

\section{Lecturing / supervision of practicals / tutorials (8.4 ECTS)}

- Frontiers in medical and veterinary biology (2014-2016)

- Biological control of insects (2014-2016)

- Molecular and evolutionary ecology (2016)

- Basics of infectious diseases (2016)

\section{Supervision of MSc students}

- Biology of potential West Nile virus vectors in the Netherlands: distribution and hostseeking behaviour

- Mating and host-seeking behaviour of Culex pipiens biotypes as vectors of West Nile virus

- Spatial patterns of mosquitoes in the Netherlands: finding hotspots of mosquito presence based on crowdsourced data

- Distribution and survival of Culex pipiens in the Netherlands

- Hybridisation and mating preference of the Culex pipiens biotypes molestus and pipiens 
The research described in this thesis was financially supported by the European Community's Seventh Framework Programme (FP7 VECTORIE project number 261466).

Cover picture: Jelke Fros

Cover design \& lay-out: Chantal Vogels

Printed by GVO drukkers \& vormgevers, Ede, The Netherlands 